\title{
Real-Time Molecular Patterns to support Intraoperative Decision-Making
}

Citation for published version (APA):

Vaysse, P-M. (2020). Real-Time Molecular Patterns to support Intraoperative Decision-Making. [Doctoral Thesis, Maastricht University]. Maastricht University. https://doi.org/10.26481/dis.20201028pv

Document status and date:

Published: 01/01/2020

DOI:

$10.26481 /$ dis.20201028pv

Document Version:

Publisher's PDF, also known as Version of record

\section{Please check the document version of this publication:}

- A submitted manuscript is the version of the article upon submission and before peer-review. There can be important differences between the submitted version and the official published version of record.

People interested in the research are advised to contact the author for the final version of the publication, or visit the DOI to the publisher's website.

- The final author version and the galley proof are versions of the publication after peer review.

- The final published version features the final layout of the paper including the volume, issue and page numbers.

Link to publication

\footnotetext{
General rights rights.

- You may freely distribute the URL identifying the publication in the public portal. please follow below link for the End User Agreement:

www.umlib.nl/taverne-license

Take down policy

If you believe that this document breaches copyright please contact us at:

repository@maastrichtuniversity.nl

providing details and we will investigate your claim.
}

Copyright and moral rights for the publications made accessible in the public portal are retained by the authors and/or other copyright owners and it is a condition of accessing publications that users recognise and abide by the legal requirements associated with these

- Users may download and print one copy of any publication from the public portal for the purpose of private study or research.

- You may not further distribute the material or use it for any profit-making activity or commercial gain

If the publication is distributed under the terms of Article $25 \mathrm{fa}$ of the Dutch Copyright Act, indicated by the "Taverne" license above, 


\section{Real-time molecular patterns to support intraoperative decision-making}


ISBN/EAN

Cover design \& layout

Printing
978-94-6423-030-7

Pierre-Maxence Vaysse

ProefschriftMaken (www.proefschriftmaken.nl)

Copyright (C) Pierre-Maxence Vaysse, Maastricht 2020 


\title{
Real-time molecular patterns to support intraoperative decision-making
}

\author{
Dissertation
}

to obtain the degree of Doctor at Maastricht University, on the authority of the Rector Magnificus Prof. dr. Rianne M. Letschert in accordance with the decision of the Board of Deans, to be defended in public on Wednesday October $28^{\text {th }} 2020$ at 10 hours by 


\section{Supervisors}

Prof. dr. R.M.A. Heeren

Prof. dr. S.W.M. Olde Damink

\section{Co-supervisor}

Dr. T. Porta Siegel

\section{Assessment Committee}

Prof. dr. R. Shiri-Sverdlov (chairman)

Prof. dr. M. Poeze

Prof. dr. A. zur Hausen

Prof. dr. N.Y.R. Agar (Harvard Medical School)

Prof. dr. G.K. Bonney (National University of Singapore)

The research presented in this thesis was financially supported by Maastricht University Medical Centre+ and by the Dutch Province of Limburg as part of the LINK program. 


\section{Table of contents}

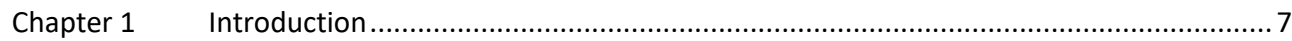

Chapter 2 Mass spectrometry imaging for clinical research ................................................. 15

Chapter 3 Multi-site round robin study of rapid evaporative ionization mass spectrometry ..... 43

Chapter 4 Stromal vapours for real-time molecular guidance of breast-conserving surgery .....65

Chapter 5 Real-time lipid patterns to classify viable and necrotic liver tumours ...................... 77

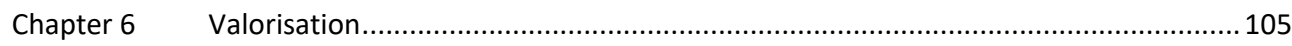

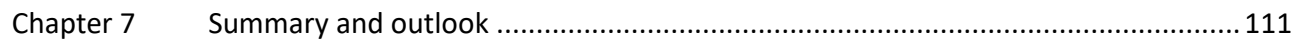

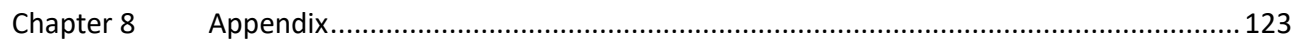



Chapter 1

\section{Introduction}




\section{Tumour hallmarks and heterogeneity}

Cancer is one of the leading causes of human death worldwide (1). Carcinogenesis is a multistage process characterised by the succession of multifactorial oncogenic insults that result in transformation of the healthy cell to tumour cell $(2,3)$. Alterations take place on multiple molecular levels; genome, epigenome, proteome, metabolome leading to a loss of homeostasis and phenotype modification (fig. 1a-b). Each of these molecular alterations influences each other and common biological changes are reported as part of the hallmarks of cancer (4) (fig. 1c). Cancer precision medicine aims to use these molecular alterations to characterise tumour phenotypes to enable better-informed clinical decision-making (5).

a)
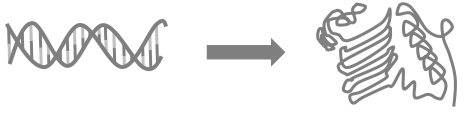

b)

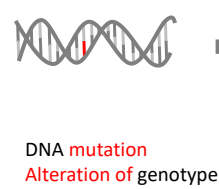

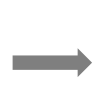

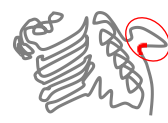

Protein mutation Loss of conformation Loss of function
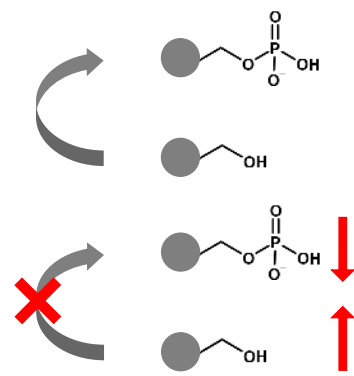

Metabolite Deficit/accumulation
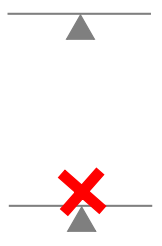

Loss of homeostasis Alteration of phenotype

c)

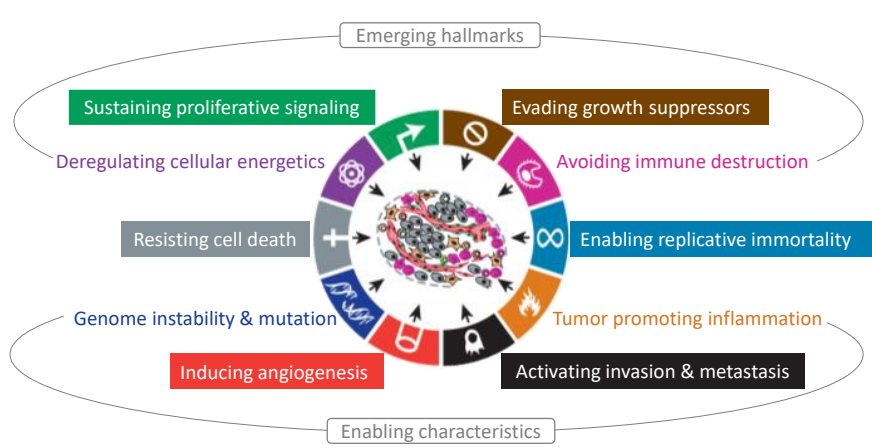

Figure 1. Tumour hallmarks. (a) Genetic mutations, protein modifications, metabolic alterations. (b) Hallmarks of cancer adapted from Hanahan and Weinberg $(4,6)$.

Beyond the molecular alterations, critical tumour characteristics that guide clinical decisions such as the tumour type, the tumour grade, or the stage of the disease are defined by the histopathological phenotype. Diverse changes are observed from a physiologically normal tissue to a tumour tissue. The specialised tissue architecture of differentiated cells required to execute specific functions is progressively lost. Precursor lesions can be recognized before the tumour generation (7). The tumour cell proliferation also impacts the surrounding healthy cells by physically pressuring the tissue structures and metabolically by consuming diverse essential nutriments for cell proliferation (fig. 2a) and leading to clinical manifestations. These changes can be tissue and cell specific. 
Despite the existence of conserved hallmarks, tumour heterogeneity is a tremendous challenge for clinical decisions (7). Successive and various stresses, genetic and environmental, lead to the formation of tumour cells specific to each patient, independently from the origin of the tumour tissue or from the histopathologic tumour type. This is referred to as intertumour heterogeneity (fig. $\mathbf{2 b - c}$ ). Moreover, similarly after the tumour generation, its development can also be influenced by general or local stresses such as radiation or drug treatment. This can result in the presence of multiple clones in the tumour tissue that can progress differently and react differently to treatment (8). This is referred to as intratumour heterogeneity (fig. $\mathbf{2 b}, \mathbf{d}$ ). Characterizing biomarkers to predict tumour progression, tumour recurrence, and treatment response are critical to take betterinformed decisions for the care of patients with cancer.

a)
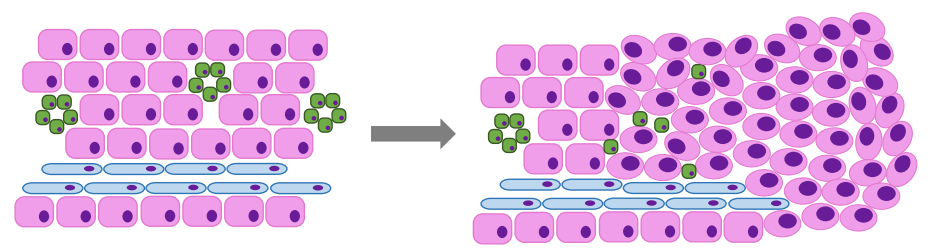

Physiology to pathology Loss of cell differentiation Loss of tissue architecture Loss of specific function

b)

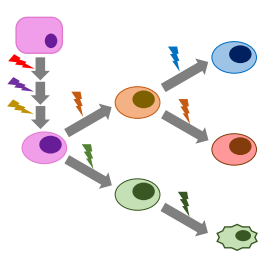

c)

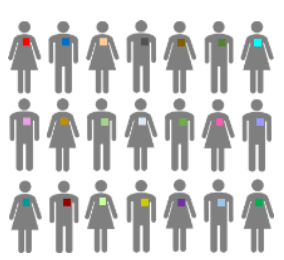

d)

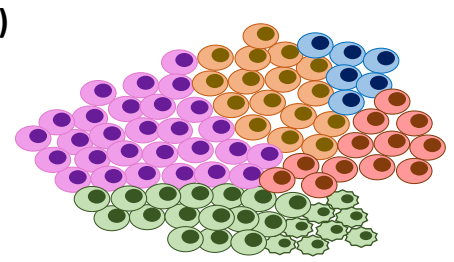

Figure 2. Tumorigenesis and tumour heterogeneity. (a) Tissue malignancy transformations. On the left, scheme of a healthy tissue with diverse cell types organised in structures (i. e. glands with green cells, ducts with blue cells) with specific physiological functions. On the right, scheme of a partially tumour tissue with proliferation of tumour cells affecting the remaining normal tissue architecture and its physiological functions. (b) Tumour heterogeneity. Diverse and successive oncologic insults can induce mutations of the cell into distinct tumour cell clones. (c) Intertumour heterogeneity. Each patient presents a specific tumour. (d) Intratumour heterogeneity. Distinct mutations of the tumour cell can lead to multiple subpopulations of tumour cell clones in one tumour tissue.

\section{Intraoperative decisions and reference diagnostics}

The journey of a patient with cancer goes through multiple steps. Clinical interventions can vary, e.g. from preventive mastectomy for women who carry a BRCA1 gene mutation, to follow-up after cancer curative treatment including chemotherapy, radiotherapy, etc. (fig. 3). Among these steps, oncologic surgery represents a keystone step in the clinical care of most patients with cancer. It aims on removing all the tumour tissues from the patient's body, while sparing healthy tissues to preserve function. The intraoperative decisions made by the surgeon to perform the resection are critical for patient survival (9-11). Unsuccessful surgeries with potential remaining tumour tissues inside the patient can lead to reoperations or heavy complementary treatments such as radiotherapy or chemotherapy affecting the quality of life and the survival of the patient. 
Adapted to the surgical specialty, diverse surgical tools can be employed to improve surgical precision ranging from electro-cautery knife in soft tissue surgeries to $\mathrm{CO}_{2}$ laser in head and neck surgeries and cavitron ultrasonic surgical aspirator (CUSA) in liver surgeries.

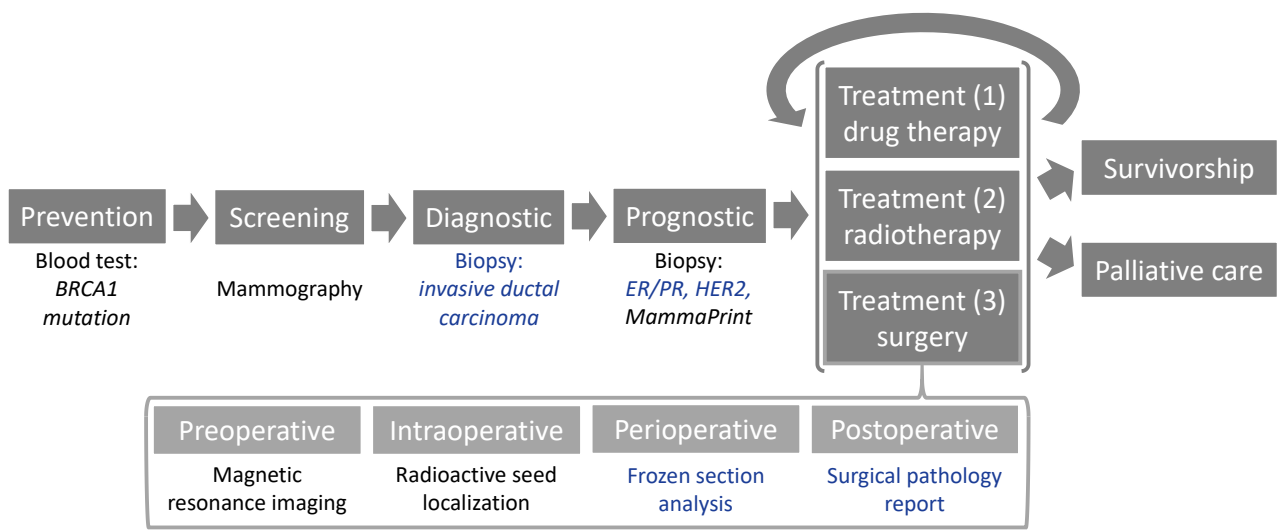

Figure 3. Diagram of the journey of the patient with cancer. Illustrations with examinations/guidance modalities and biomarkers employed for the management of patients with breast cancer (12-15). Examinations involving histopathology indicated in blue. BRCA1: breast cancer type 1, ER: oestrogen receptor, PR: progesterone receptor, HER2: human epidermal growth factor 2.

Macroscopic distinction between tumour tissues and healthy tissues can represent a challenge during the operation. Therefore, surgery can be assisted by preoperative, intraoperative (in the operating room) and perioperative (outside of the operating room) and postoperative modalities $(12,16,17)$. Among these modalities, computed tomography and magnetic resonance imaging are employed to prepare before lung cancer surgery, ultrasound and radioactive seed localisation can be used intraoperatively to guide breast cancer surgery, and histopathology, gold standard for the diagnostic of solid tumour tissues, can come into play with perioperative frozen section and postoperative surgical pathology report.

The pathology diagnostic requires processing of the tissue specimen (fig. 4). This includes fixation, sectioning, staining and microscopic examination of the tissue architecture by a trained pathologist. The most conventional staining to enhance the contrast of cell morphologies is based on a stain of haematoxylin which colours nucleus in violet and eosin, which colours cytoplasm in pink. Specific histologic features can be visualised using specific stains or targeted markers for immunohistochemistry. The variety of tissues and tumours encountered in the clinics has led the medical specialisation to specialised training of professionals for the recognition of pathological features to enable diagnostics (fig. $\mathbf{5 b}$ ). Guidelines are elaborated worldwide to characterise tumour characteristics such as type, subtype, grade or response to therapy (18). Nevertheless, diagnostic subjectivity (19) can lead to disagreement between experts (20). 


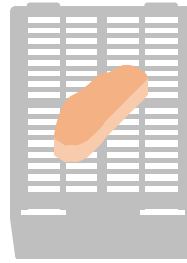

Tissue

fixation

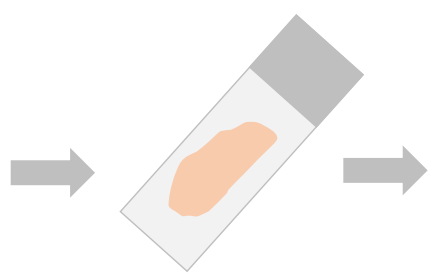

Tissue sectioning

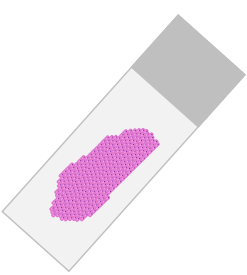

Histological staining

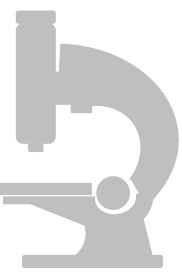

Microscopic examination

Figure 4. Histology workflow required before histopathology examination. It includes fixation (e.g. freezing, formalin), sectioning, staining and microscopic examination.

During the oncologic surgery, when the surgeons are unsure about the malignant nature of the tissue, they can request a pathology examination by frozen section (21). The tissue has to be frozen for an optimal time constrained fixation (i. e. immersion in liquid nitrogen for a few seconds), sectioned and stained for pathology examination. The histopathologist has to give feedback on the malignancy of the tissue for the surgery procedure to carry on, while frozen sections can be challenging to interpret with certainty. Postoperative surgical pathology assessment on the full specimen enables more certain and precise conclusions and can be incongruent.

In practice, the tumour tissue can be resected with a surrounding of normal tissue to be sure to remove all the tumour tissues. Therefore, at the end of the resection, surgeons sometimes indicate the orientation of specimen with stitches (fig. 5a). Based on the orientation, pathologists apply one or a few inks on the resection margins of the full surgical specimen before eventual additional slicing in the specimen. Tissues are then fixed in formalin for hours for optimal histology and long term storage at room temperature.

The distance between the tumour and the resection cut is referred as resection margin width and has been reported as an independent prognostic factor on patient's outcome for several malignancies $(9-11,22)$. The distance between the inked margin and the first encountered tumours cells are then inspected microscopically to define the success of the surgery (fig. 5c). Tissue handling, processing and examination take days before providing a definitive pathology report on the surgical resection, which delays clinical decisions that can affect patient outcome. Fast pathology assessment during surgery would benefit to the management of patients with cancer.
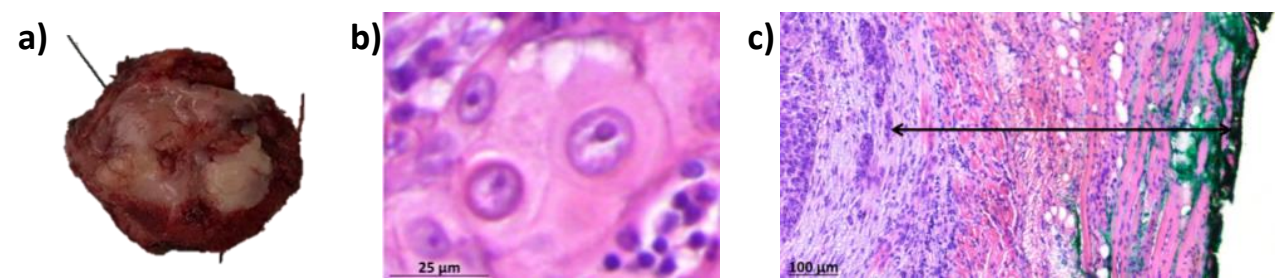

Figure 5. Surgery and histopathology: example of tongue cancer tissues. (a) Macroscopic pathology examination of a surgically resected specimen. (b) Microscopic pathology examination for tumour diagnostics: morphological features of oral squamous cell carcinoma. (c) Microscopic pathology examination of resection margin: distance between the first tumour cell and the resection margin inked in green. 


\section{Imaging and real-time molecular patterns}

Mass spectrometry is an analytical technique that separates charged molecules (ions) based on their mass-to-charge ratio $(\mathrm{m} / \mathrm{z})$ and measures their relative abundance. It encompasses three steps: ionization, separation and detection. Commonly preceded by separative techniques (23), its broad utilisation in diverse fields such as drug development, quality control or toxicology aims at the structural characterisation of compounds (24). Its utilisation without separation beforehand for fast characterization of biological tissues only emerged in the past decades (25).

Mass spectrometric patterns generated from tissue sections demonstrated their specific distributions along histopathological features in dimensional space, referred as mass spectrometry imaging (MSI). After proving the tissue type specificity of molecular patterns to classify tumour tissues (26), the MSI field evolved driven by technical improvements (27). Initial applications on biological investigations were mainly investigating protein distributions before the emergence of ambient ionization sources (28) and lipid applications announcing the trend towards the speed for surgical applications.

The combination of mass spectrometry with direct sampling probes (29-31) enabled fast tissue analyses without tissue sectioning required for MSI. Rapid evaporative ionization mass spectrometry (REIMS) (fig. 6) is one of these ionization sources that enable tissue recognition in a few seconds based on lipid patterns. These real-time tissue classifiers can provide a new way to assess tissue pathology for faster intraoperative decisions, critical for patient's outcome.

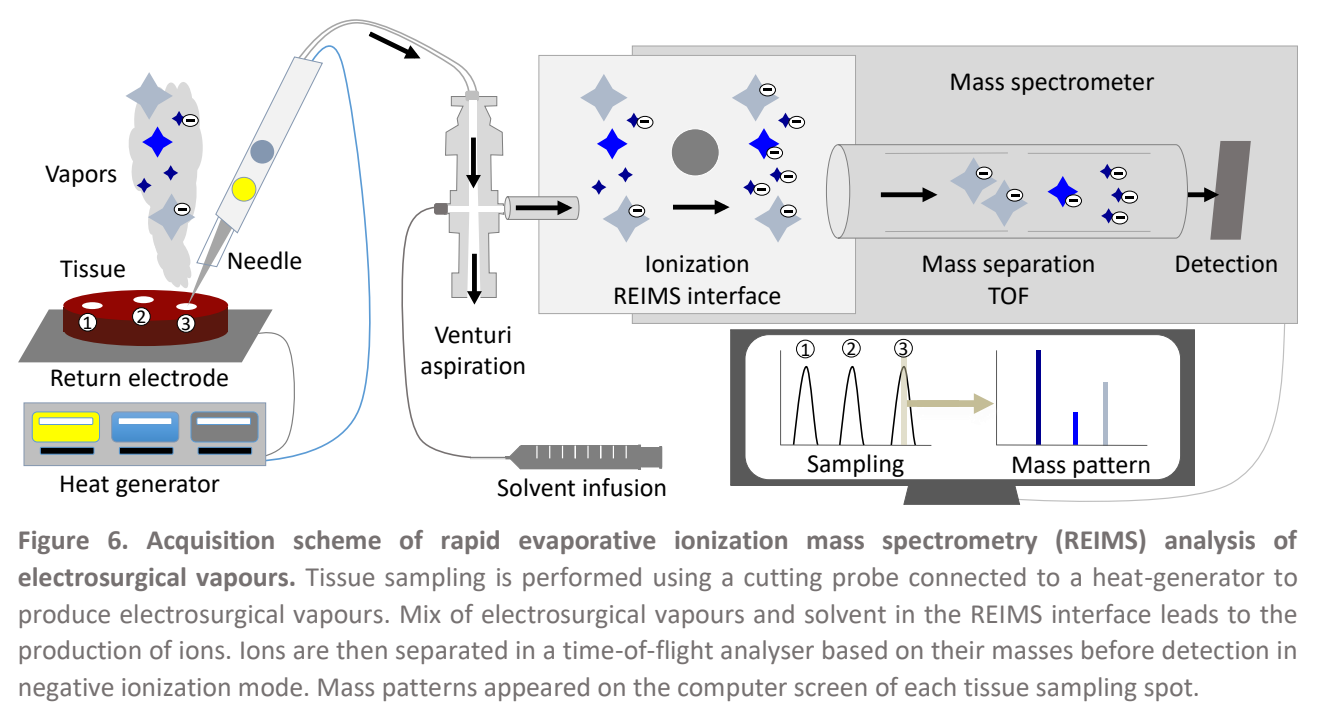

\section{Scope and aims}

This thesis investigates the potential of the emerging mass spectrometry technology REIMS, designed to classify tissues in real-time based on lipid patterns. The study is focused on the 
application of REIMS on tumour resected specimens from patients undergoing oncologic surgeries, in correlation with histopathology examination.

The main aims were: 1 ) to review the milestones of mass spectrometry imaging (MSI) that have led to consider molecular patterns reliable to characterise tissue pathologies, before the emergence of direct sampling technologies, 2 ) to assess the reproducibility of REIMS for the classification of biological tissues, 3) to enable in vivo tissue recognition during surgery based on a library of lipid patterns validated by histopathology, and 4) to explore the association between lipid patterns and liver tumour heterogeneity.

\section{Outline}

In chapter 2, the latest developments, applications and current limitations of MSI in clinical research are reported to overview the past directions taken by the research field and its next challenges towards providing new tools for better informed clinical decisions based on molecular information. Within the framework of a multi-site round robin international collaboration, chapter 3 demonstrates the reproducibility of REIMS for the classification of biological tissues including food samples and breast tumour resected tissues validated with histopathological examination. In chapter 4, a library of ex vivo lipid patterns based on histopathology examination is built to enable in vivo tissue recognition during breastconserving surgery. The importance of intraoperative precise tissue classification is emphasized by the potential of stromal vapours for surgical guidance. Chapter $\mathbf{5}$ demonstrates the potential of lipid patterns to provide metabolic information reflective of tumour heterogeneity to support decision-making in cancer precision medicine. Classification of liver parenchyma, primary and metastatic tumours is achieved on liver resection tissue and the heterogeneity of these tumour lipid patterns can be associated to the histopathological phenotype. Moreover, ceramides and related sphingolipids discriminate necrosis from viable tumour parts and contribute to new tumour classifications of based on metabolic shifts. Chapter 6 discusses the findings exposed in thesis with regards to their potential societal impact, beyond the research purpose only. Chapter 7 summarizes the main findings of this thesis and put them in perspectives with future research investigations and their clinical and biological implications. 

Chapter 2

\section{Mass spectrometry imaging for clinical research}




\begin{abstract}
Mass spectrometry is used in many clinical research areas ranging from toxicology to personalized medicine. Of all the mass spectrometry techniques, mass spectrometry imaging (MSI), in particular, has continuously grown towards clinical acceptance. Significant technological and methodological improvements have contributed to enhance the performance of MSI recently, pushing the limits of throughput, spatial resolution, and sensitivity. This has stimulated the spread of MSI usage across various biomedical research areas such as oncology, neurological disorders, cardiology, and rheumatology, just to name a few. After highlighting the latest major developments and applications touching all aspects of translational research (i.e. from early pre-clinical to clinical research), the present challenges in translational research performed with MSI is discussed: data management and analysis, molecular coverage and identification capabilities, and finally, reproducibility across multiple research centres, which is the largest remaining obstacle in moving MSI towards clinical routine.
\end{abstract}

\title{
Introduction
}

Biomedical research relies on the investigation and analysis of biological specimens such as body fluids and solid tissues (32). To obtain comprehensive molecular information from these samples, omics technologies are frequently employed. Amongst these methods, mass spectrometry (MS) constitutes a powerful and versatile technique for the unlabelled and multiplexed analysis of the non-genomic and non-transcriptomic information from a broad variety of molecules ranging from metabolites (metabolomics) to proteins (proteomics) to pharmaceutical compounds (33-35).

However, when it comes to spatially complex samples such as solid tissues or alike specimens (e.g. organoids), it is crucial for a correct biological interpretation to be able to assign the detected molecular information to the individual cells or surrounding areas such as extracellular matrix. Therefore, methods are required that account for both the spatial structure and cellular heterogeneity of the sample.

For this purpose, most analytical workflows make use of isolation steps of the cellular material of interest, such as microscopic dissection (micro-dissection) or other cellenrichment procedures preceding the omics analyses $(36,37)$. These methods provide unrivalled analytical depth and sensitivity, but are tedious, time consuming, and/or require previous knowledge of the cellular entities of interest; and some may even destroy the histological context of the sample.

Mass spectrometry imaging (MSI) is a microscopic technique that can directly analyse tissue sections without the need to destroy sample integrity (38). All MSI-based techniques rely on the discrete or continuous movement of a desorption and ionization probe across a sample's surface (39). At each measurement coordinate, molecules present at the sample's surface are desorbed and ionised, and a mass spectrum is recorded along with its current position. This enables plotting images that show the spatial distribution and abundance of a selected $\mathrm{m} / \mathrm{z}$ species, and therefore molecule, in the sample. To do so, the intensity values of a selected $\mathrm{m} / \mathrm{z}$ species (or $\mathrm{m} / \mathrm{z}$ interval) are extracted from each acquired mass spectrum and then arranged into an image based on the original positions of the respective mass 
spectra (fig. 1a-b). After the measurement, an optical image of the very same sample can be taken and coregistered with the MSI data (fig. 1a,c).

The ability to combine MSI data with histological and morphological information is critical for biomedical research (40). This allows not only a view of molecular distributions in their histological context, but also use of the histological information to define regions-ofinterest (virtual micro-dissection) that harbour the cells of interest in order to extract their characteristic patterns from different molecular classes such as metabolites and proteins (fig. 1c-d) (41).

a)

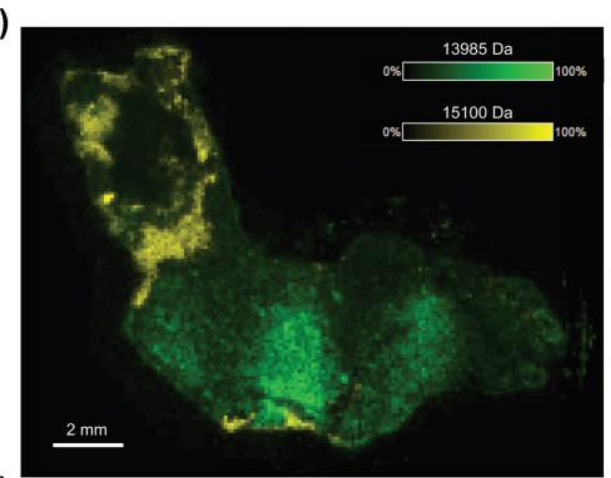

c)

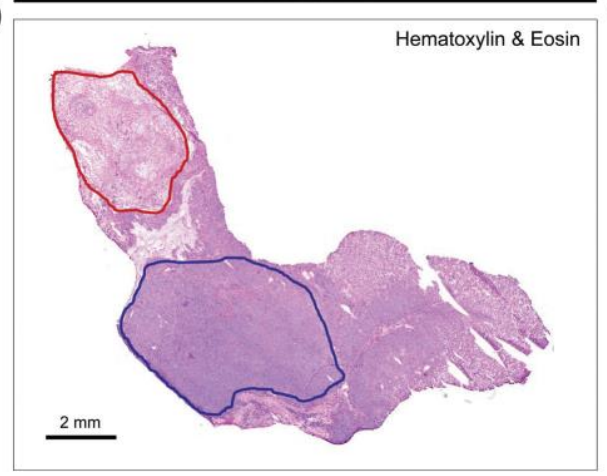

b)

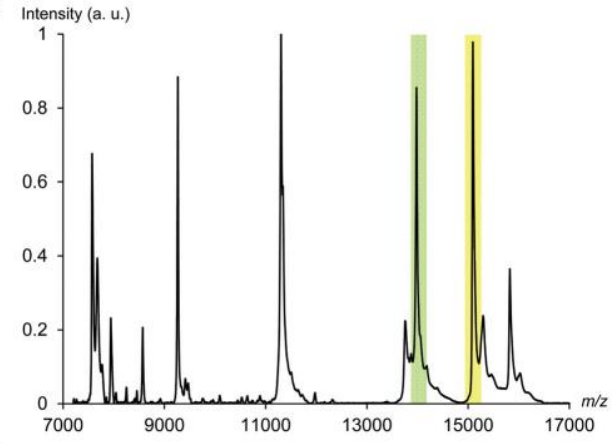

d)

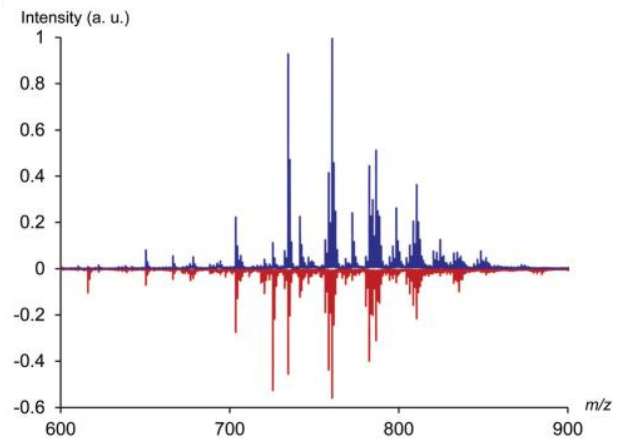

Figure 1. Mass spectrometry imaging (MSI) of a tumour resected specimen. (a) Visualization of the intensity distributions of two mass signals belonging to two proteins with masses between 14000 and 16000 Daltons (b) in a tissue section of the sample. (c) After acquisition the very same tissue section can be stained and its microscopic image coregistered to the MSI data. The histological information can be used for manual annotation of regions. Here, the region marked in red demarks necrotic tumour and the region in blue vital tumour with infiltrating inflammatory cells. (d) These annotations can be either used to extract molecular patterns from regions of interest or to guide subsequent experiments for a local MSI analysis or liquid extraction.

These cell-type specific molecular patterns increase the specificity of the investigation, making MSI an attractive tool in clinical research. This is especially true for cancer research where MSI has made most of its contributions to biomarker discovery, description of interand intratumour heterogeneity, and high-throughput profiling to complement established histopathological diagnostics (42). The latter has especially benefited from the rise of ambient ionization methods, which usually do not require sample preparation, and accelerated MSI instrumentation (43). Both innovations now enable the analysis of samples 
at time scales that could be compatible with clinical practice. Furthermore, protocols have been developed to make MSI compatible with routine clinical tissue processing, such as formalin-fixed, paraffin-embedded specimens $(44,45)$. Thus, MSI is not only considered a powerful biomedical research tool, but also has the potential to become a clinical assay itself.

In this chapter, the history of MSI in biomedical research is reviewed in the light of the continuous technical developments and improvements over the last 10 years, which were stimulated by biomedical research and clinical needs. Existing limitations of MSI are discussed, which highlight efforts that MSI the community must undertake to drive this technology further, especially for clinical research and related applications.

\section{MSI imaging techniques}

Mass spectrometry imaging (MSI) was developed in the early 1960s with the introduction of secondary ion mass spectrometry (SIMS) (46). In SIMS, a pulsed ion beam moves across the sample's surface and due to its impact secondary ions are generated locally and then directed to the mass spectrometer inlet (fig. 2a). This technique has been used for imaging the elemental and molecular composition of surfaces at spatial resolutions in the subcellular scale (fig. 2d) (47).

It was not until the mid- and late 1990s, when MSI based on matrix-assisted laser desorption/ionization (MALDI; fig. 1b) was applied to biological tissue sections, which are placed as sections on a target plates (25) or a few years later on (conductive) glass slides (48). Using MALDI as a soft ionization technique, it became feasible for the first time to image the distribution of larger biomolecules in tissues (fig. 2e) $(25,49)$. Therefore, most commercial MALDI-MSI systems use a time-of-flight mass analyser.

In contrast to SIMS, MALDI uses a laser to probe a specific location on a tissue's surface (fig. 2b). MALDI-MSI requires the application of an organic matrix to act as a mediator between the laser energy and the analytes and to promote their ionization. For a successful MALDI imaging experiment, homogeneous matrix deposition across the tissue's surface is crucial, and can be accomplished by spraying, spotting, or sublimation. While this was done initially by hand-held sprayers, biomedical research questions quickly demanded the reduction of variance-within and between samples-through automation of the matrix deposition. Several commercial systems are now available (e.g. Bruker ImagePrep, SunChrome SunCollect, HTX TM-Sprayer, tardo iMatrixSpray, TransMIT SMALDIPrep), which differ in their condition control (temperature, oxygen level), homogeneity of the matrix coating, and average matrix crystal size, all of which determine the experimental limitations (50). The matrix crystal size, for instance, defines the achievable spatial resolution (51), and environmental conditions together with the chosen matrix and solvent influence the extraction capabilities and therefore the molecular class that can be investigated.

Proteins were the first molecular class to be visualized by MALDI-MSI (25). By the use of other matrices and sample preparation, MALDI-MSI has been quickly extended to other molecular classes such as peptides, lipids, and small molecules including exogenous compounds like drugs (fig. 2e) (53). For each of these molecular classes, specific sample preparation protocols have been optimized to improve their detection. These optimizations include solvent washes and recrystallization for low abundance intact proteins (54), 
enzymatic on-tissue digestion for peptide imaging (55), and in situ derivatization of analytes in order to increase their ionization efficiency (56). Due to its versatility in terms of spatial resolution and molecular coverage, MALDI-MSI has become the most popular MSI technique over the past few years and has been successfully used in many clinical research studies, of which the most important examples are presented in this chapter. However, MALDI-MSI suffers from a chemically intensive sample preparation, which is time consuming and can be a source of artefacts $(57,58)$. This drawback has led to the development of other imaging-compatible ionization techniques.

a)

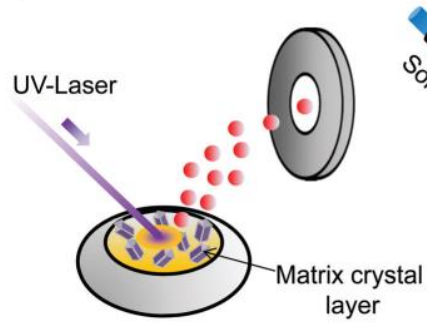

d)

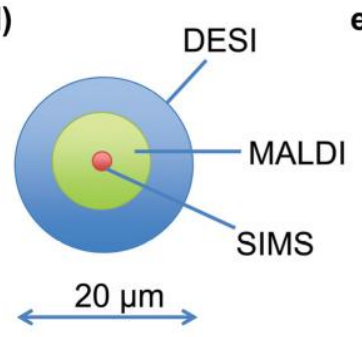

e) b)
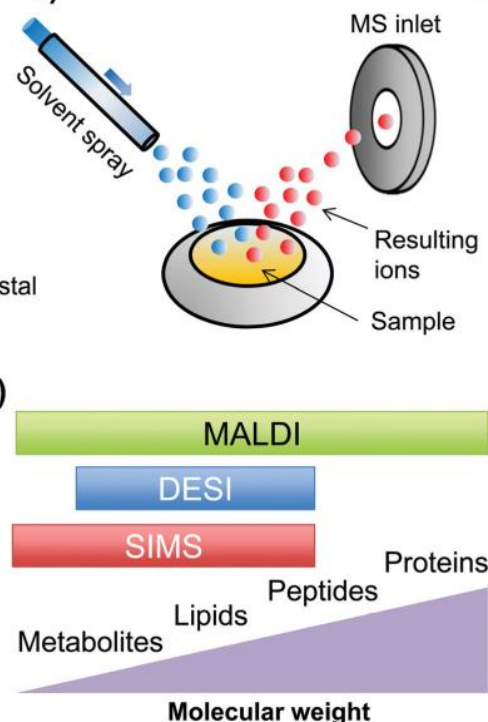

c)

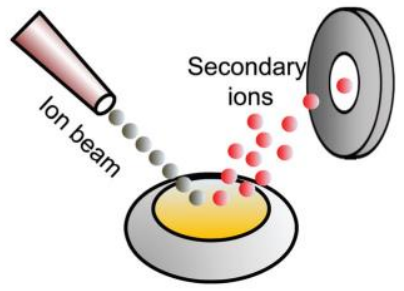

f)

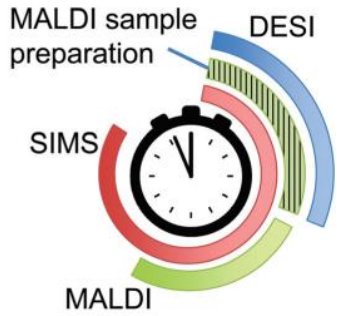

Figure 2. Common mass spectrometry imaging (MSI) technologies. (a) Matrix-assisted laser desorption/ionization (MALDI) MSI requires applying an organic matrix across the sample in such a way that the analytes are locally extracted and incorporated into the matrix. When the laser hits the sample at a certain position, the MALDI process generates ions that are analysed by the mass spectrometer (MS), generating a spectrum, which is associated with that position. The acquisition of spectra in a grid-wise manner enables creating ion distribution maps. (b) Desorption electrospray ionization (DESI) MSI works in a similar way, with the difference that, instead of a laser, a solvent spray is directed towards the sample for desorption. As the solvent comes into very brief contact with the sample, it extracts the analytes locally and transports them to the mass spectrometer inlet. DESI does not require a matrix and hence no sample preparation. (c) Secondary ion mass spectrometry (SIMS) imaging uses pulsed ion beams to locally desorb compounds from a certain position. (d) The SIMS ion beam has the smallest diameter; hence it offers the highest spatial resolution, followed by MALDI and DESI due to their larger laser and solvent spray diameters, respectively. (e) MALDI-MSI, however, offers the highest versatility in mass range and hence type of investigable molecular class, ranging from small molecules to biological macromolecules such as proteins. (f) DESI is overall the fastest MSI method, but net (i.e. without sample preparation) MALDI performs equally. (a), (b), and (c) are reprinted with permission from Addie et al (52). Copyright (2015) American Chemical Society.

Desorption electrospray ionization (DESI) is one of the first ionization sources to allow imaging under ambient conditions (59). In DESI, a solvent of charged droplets is directed toward the surface, which first leads to desorption of the analytes followed by their ionization based on the same principle as electrospray ionization (fig. 2c). Similar to the laser 
in MALDI-MSI, the spatial resolution of a DESI-MSI experiment is partly determined by the diameter of the aerosol hitting the surface of the sample (fig. $\mathbf{2 d}$ ). As the spray is applied continuously and the sample stage moves continuously, the horizontal pixel size is defined by the analyser scan time during which the sample stage moves a certain distance (60). With the introduction of high-repetition rate lasers, a quasi-continuous situation is attained which significantly improved MALDI-MSI acquisition speed. The horizontal pixel size is defined by the stage motion, laser frequency, and a parameter defining the laser shots to be averaged per pixel (61). In both cases, the distance between two horizontal line scans determines the vertical pixel size.

With respect to the molecular classes that can be investigated, DESI-MSI experiments from tissues have been mainly reported on small molecules such as metabolites (e.g. neurotransmitters), drugs, and lipids (fig. 2e) (62-64). While changing the spray solvent composition can selectively promote the detection of certain molecules, the spray solvent can also be loaded with a derivatization agent (65). In this so-called "reactive-DESI" set-up, while the charged droplets hit the surface, a short and selective chemical reaction can take place between the derivative agent and the target analytes to improve their detection. The ability to study samples with minimal or no sample preparation makes DESI-MSI a valuable tool for high-throughput MSI and therefore especially interesting for large sample cohorts encountered in clinical research (fig. 2f) (66).

DESI has opened the way for a variety of other kinds of ambient ionization techniques. However, their current impact regarding clinical research is at present limited, as they are focused on their technical development or have been recently introduced to the field. Nano-DESI (nanospray desorption electrospray ionization), for instance, improves DESI by substituting the charged aerosol with a continuous micro-extraction realized by a liquid bridge of solvent between two capillaries, leading to higher spatial resolutions of up to 12 $\mu \mathrm{m}(67)$ and the capability of protein analysis (68). LESA (liquid extraction surface analysis) was reported for studying distributions of a wide range of analytes, including proteins, at the millimetre scale. LESA relies on the formation of a liquid micro-junction with the tissue and allows static extraction of analytes $(69,70)$. LAESI (laser-ablation electrospray ionization) combines a mid-infrared laser for desorption and an orthogonal electrospray for transferring multiple charges to the analytes. This promises to extract larger biomolecules such as peptides and small protein with subsequent MS/MS identification in a single run (71). Finally, it is also worth mentioning the matrix-free laser methods LA-ICP (laser-ablation inductively-coupled plasma), which enables analysing elemental distribution in samples (72), and NIMS, (nanostructure initiator mass spectrometry)(73) another matrix-free softionization technique.

The three main techniques MALDI, DESI, and SIMS are compared in fig. $\mathbf{2}$ according to their working principle, achievable spatial resolution, molecular coverage, and experimental time investment, all of which are important for biomedical research studies. 


\section{Latest technical developments in MS imaging}

\section{The quest for high throughput}

Clinical research usually implies the analysis of hundreds of patient samples, representative of large clinical cohorts. To address clinical questions at relevant time scales, recent developments have enabled high throughput analysis with unprecedented data acquisition speeds (74).

While most MALDI-TOF instruments use a spot-by-spot sampling technique in which the stage moves in discrete steps under a stationary laser, recently developed systems utilize continuous laser raster sampling to acquire data while continually firing the laser and scanning the stage $(75,76)$. The performance is further improved by faster laser repetition rates, improving the acquisition rate to 50 pixels per second (50 times faster than that available in 2006) (fig. 3a) $(77,78)$.

To illustrate, if one wants to image a surface area of $10 \times 10 \mathrm{~mm}$ at a spatial resolution of 50 $\mu \mathrm{m}$ (i.e. with a pixel size of $50 \times 50 \mu \mathrm{m}$, which is a total of 40000 pixels), it would almost take six hours using instruments operating at an acquisition speed of two pixels per second. On the other hand, the same experiment performed at an acquisition speed of 50 pixels per second would only take 13 minutes.

Spraggings et al. obtained the distribution of lipids in a sagittal rat brain tissue section within 10 minutes, obtained from high speed MALDI-TOF MSI experiments combining high sample stage velocity of $5 \mathrm{~mm} / \mathrm{s}$ in continuous laser raster mode with a high laser repetition rate of $3 \mathrm{kHz}$ (61). Using MS/MS, Prentice et al. were able to image drugs and common kidney lipids in a complete section in less than 20 minutes at a spatial resolution of $100 \mu \mathrm{m}$ (78). With this next-generation of high-speed MALDI-TOF MSI systems, it is now even possible to use volatile matrices, which can improve sensitivity for certain molecular classes compared to commonly used matrices (75). Combined with the use of tissue microarrays, which can concentrate dozens to hundreds of patient biopsies on one glass slide, a throughput of thousands of patients in a few days has now become feasible (fig. 3a,c) (79).

Although major improvements reported so far are related to MALDI-MSI, with respect to speed recent developments have put DESI-MSI in a competitive position, with acquisition speed capabilities of 30 pixels per second (80). In addition, the absence of time-consuming sample preparation contributes considerably to reducing the overall analysis time (fig. $\mathbf{2 f}$ ).

Altogether, these improvements offer the possibility to answer clinical questions in short time frames at high spatial resolution.

\section{Imaging at cellular length scales}

Tissue is a highly complex system at the cellular level (81). High-resolution imaging combined with molecular specificity would thus be extraordinarily beneficial for a better understanding of the construction of a cellular system, especially in small-scale samples such as biopsies or tissue microarrays cores (fig. 3b). Developments in sampling techniques have enabled for imaging at cellular length scales (82). In cancer, for instance, this allows for a more differentiated molecular analysis by separating tumour cells from stromal components (83) or inflammatory infiltrates (84). 
In this sense, SIMS is particularly attractive for sub-micrometre resolution imaging (85), but at the expense of long acquisition times. Advances in ion probe technologies have increased the dynamic range and sensitivity of SIMS, allowing 2- and 3-dimensional localization of analytes in a variety of cells $(86,87)$. In 2011, Brison et al. used SIMS to create molecular depth profiles of human HeLa cells treated with the anticancer drug bromodeoxyuridine allowing to image molecular and atomic species from BrdU inside the nuclei of the cells (88).

While current commercial MALDI-MSI instrumentation allows the acquisition of $10 \mu \mathrm{m}$ pixels, $5 \mu \mathrm{m}$ MALDI imaging without oversampling has recently been reported (89). MALDI sources operating at atmospheric pressure allow for the return of subcellular pixel sizes below $2 \mu \mathrm{m}(90,91)$. This is possible by focusing of the laser beam, which consists of a prefocusing part outside a vacuum and a final focusing part within the vacuum directly above the sample manipulator $(90,91)$. However, decreasing the size of the sampled area affects the sensitivity negatively and there is a compromise to make between resolution and sensitivity (92).

Laser-ablation inductively coupled plasma mass spectrometry (LA-ICP-MS) offers nowadays a lateral resolution down to $1 \mu \mathrm{m}$ and has been applied in biomedical research such as epitope-based MSI (72).

\section{Epitope-based MSI}

Targeted epitope-based MSI utilizes laser cleavable mass tags bound to specific antibodies for detecting multiple antigens in a single tissue section (93). Imaging mass cytometry, based on LA-ICP-MS, has emerged as a highly multiplexed epitope-based imaging approach that uses rare earth metals as reporters on antibodies enabling high-dimensional, singlecell imaging of cell type and state (94). Bodenmiller et al. demonstrated the simultaneous imaging of 32 proteins and protein modifications at subcellular resolution in human breast cancer samples, allowing delineation of cell subpopulations and cell-cell interactions and highlighting tumour heterogeneity (95). The advantage of this approach compared to untargeted MSI is its increased sensitivity and specificity for the price of being able to target antigenic proteins only.

\section{Advances in sample preparation}

MSI experiments have benefited from technical advancements at the MS instrumentation level. However, sample preparation is equally important, and therefore techniques and protocols have also been improved to match the capabilities of new MS instrumentation. It has been shown that optimized sample washing protocols can significantly improve the overall spectral quality of certain molecular classes such as lipids or proteins (96-100). Furthermore in MALDI-MSI, new matrix deposition techniques that provide smaller crystal sizes and decrease diffusion were necessary to match the reduced laser focus. Today new matrix coating systems, such as sublimation devices, offer crystal sizes of less than $3 \mu \mathrm{m}$ $(54,101)$. Also, pre-coated glass slides were introduced that decrease sample preparation times and batch effects due to temporally very distant matrix coating procedures (102). Sample preparation protocols were further developed to enable the detection of previously unexploited molecular information. 
a)

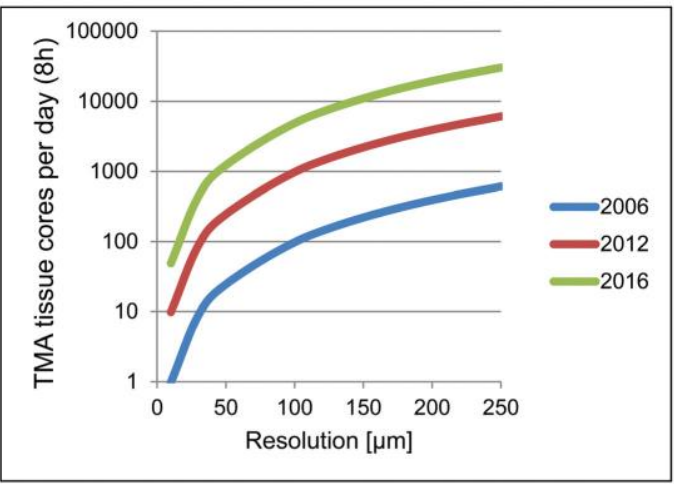

b)

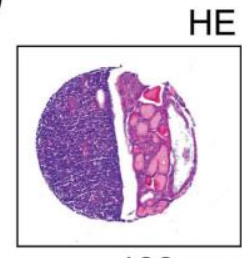

$100 \mu \mathrm{m}$

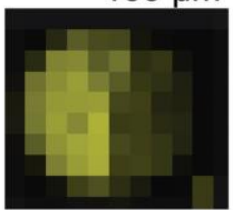

$50 \mu \mathrm{m}$

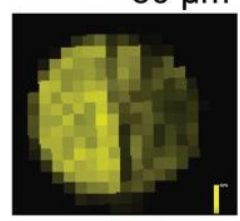

$200 \mu \mathrm{m}$

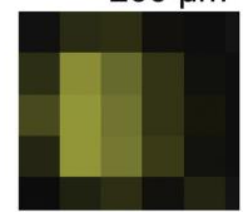

c)
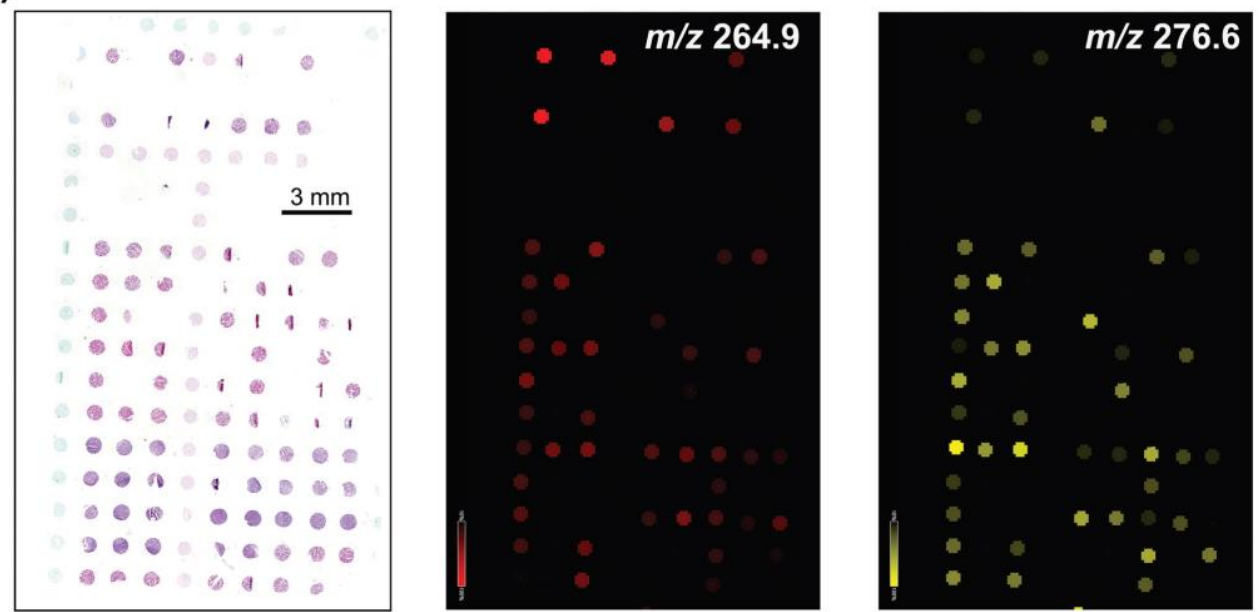

Figure 3. High-throughput character of MSI. (a) Technical developments in the last ten years have sped-up MSI systems by one and a half orders of magnitude, as shown here for the number of analysable tissue cores per eight hours as a function of spatial resolution. (b) Tissue cores are punch-outs from bigger tissue samples (usually formalin-fixed paraffin-embedded tissue blocks) with a diameter of 0.5 to $2 \mathrm{~mm}$. As it can be seen in the depicted example (scale bar $=400 \mu \mathrm{m}$ ), high resolution scanning is recommended (i.e. here around $50 \mu \mathrm{m}$ ) to distinguish histological details within a tissue core. (c) The experimental throughput can be increased by analysing a tissue microarray (TMA) which can contain hundreds of tissue cores - i.e. hundreds of patients - on one microscopic glass slide $(75 \times 25 \mathrm{~mm})$, as shown in the left panel. This allows correlating differential patterns of molecular species within the clinical data of the patient tissue cores present on the TMA. Examples of visualization of two small molecules are shown in the middle and right panel. Abbreviations used: H\&E, haematoxylin and eosin staining.

Sample preparation can be specifically optimized by the use of on-tissue derivatization agents. For target analytes such as drugs, derivatization can be extremely useful to increase their ionization efficiency and/or to avoid background interferences by shifting their $\mathrm{m} / \mathrm{z}$ signal in the spectrum. Barre et al., for example, used Girard reagent $T$ to derivatize triamcinolone acetonide, a drug employed to ameliorate the inflammation and pain of osteoarthritis (103). Recently Liu et al. used diethyldithiocarbamate to enhance signal of platinum-based drugs, which are widely used anti-cancer agents (104). But derivatization can also be beneficial to enhance the signal of a group of molecules of interest. Esteve et al. used three derivatives to enable imaging of 23 amino metabolites distributions such as 
glutamate in mouse brains (56). And Franck et al. have improved on-tissue protein identification by derivatizing $\mathrm{N}$-terminal peptides, which increased fragmentation yields significantly (105). Finally, the spray solvent of reactive DESI-MSI was loaded with a derivatization agent to promote a selective and short chemical reaction for signal enhancement of cholesterol in brain tissue (106).

Another important on-tissue chemistry is digestion, in which an enzyme is deposited onto the tissue's surface. In situ proteolytic digestion is key in the proteomic analysis of formalinfixed paraffin-embedded (FFPE) tissues, which constitute the vast majority of archived tissues in a clinical setting. The development of methods for the reversal of the formalinfixation in order to maximize the obtainable proteomic information has hence been of interest to many MSI groups with clinical aspirations (107-109). These different approaches have been further investigated, compared, and optimized in terms of data quality, tissuetype specific dynamics, and reproducibility (110-113). The use of enzymes is not only restricted to the aim of detecting peptides, but also specific post-translational modifications. Glycosylation is one of the most important protein modification involved in cell signalling and recognition. The use of PNGase $F$ to gain access to glycans has been transferred to MSI of frozen and FFPE tissues and shown to reveal biomedically relevant information (114,115). First, Holst et al. combined derivatization and subsequent PNGase digestion to distinguish the distribution of glycan enantiomers in sarcoma tissues (116). Shortly later, Heijs et al. sequentially applied different enzymes on the very same FFPE tissue section to obtain both information on $\mathrm{N}$-glycans and peptides (117).

These approaches show that the molecular information contained in FFPE tissues on a proteomic and protein-modification levels is rich and can be extracted using the appropriate techniques. Recently Walch et al. demonstrated that metabolic information can also be extracted from clinical FFPE tissues using high-mass resolution MSI (45). Running against the assumption that metabolites are washed out during tissue fixation, many metabolites with clinical value are retained (118).

\section{Advances in mass resolution, accuracy, and selectivity}

MSI has not only advanced in terms of speed, spatial resolution, and sample preparation, but has also profited from general improvements in mass analyser technologies in order to increase mass resolution and mass accuracy.

Time-of-flight. Most MALDI-MSI instruments are still based on time-of-flight (TOF) mass spectrometry (MS). TOF-MS offers a high mass range and high throughput, and latest developments have contributed to increasing its performance in terms of mass resolving power and mass accuracy. Particularly, the introduction of orthogonal acceleration geometry (oa-TOF) allows for hybridization between TOF and quadrupole mass analysers, i.e. QqTOF have been demonstrated to be particularly suited to the analysis of small molecules, with mass resolving power up to 50,000 and with MS/MS ability (119-121). TOF/TOF instrumentation that enables multiple fragmentation events to be performed in a single laser shot was also demonstrated to be beneficial for on-tissue quantitation of drugs (122).

Fourier-transform ion cyclotron resonance mass analysers. Fourier-transform ion cyclotron resonance mass spectrometry (FT-ICR MS) provides the highest mass resolving power (>> 
100,000) and accuracy (< $1 \mathrm{ppm}$ ) and allows for multistage MS/MS capabilities.(123) FT-ICR MS is not routinely used for high throughput/resolution imaging due to the long in-cell accumulation required. But the use of FT-ICR MS for high mass resolution MS imaging has enabled imaging of mass channels with a bin size as low as $\Delta m / z=0.005$, thereby revealing new features that cannot be resolved with lower resolution instrumentation (123-126). The increased mass accuracy provided by FT-ICR MS has also improved identification capabilities in MSI (see section on identification strategies below).

Tandem mass spectrometry. Tandem mass spectrometry provides additional information on the structure of the analysed molecules based on detecting their fragments. In MSI, tandem MS has been performed directly from tissue to identify peptides or lipids using either TOF/TOF, Q-TOF, or Orbitrap systems $(91,127,128)$. Tandem MS is also particularly suited for targeted imaging and offers unique capabilities in monitoring specific ion fragment transition(s) ("selected reaction monitoring", SRM) which provides unprecedented selectivity and therefore sensitivity for given compounds. Despite their lower mass resolution, quadrupole and ion trap mass analysers are particularly suited for targeted imaging of small molecules $(62,129)$. Hence, especially drug imaging studies have benefitted from the high selectivity and speed of SRM imaging on MALDI and DESI systems (60,130-132). But recently also parallel MS1 and MS2 has been introduced for SIMS (133).

lon mobility. The implementation of a post-sampling and post-ionization gas-phase separation technique in an MS imaging workflow is advantageous to differentiate isobaric analytes based on their different structures and also to discriminate between ions of interest and interfering biological matrix ions (134). The interest in ion mobility separation (IMS) in MSI has therefore increased considerably over the last few years (135). IMS has proven suitable for the analysis of various analytes, from small drugs (136), carbohydrates, and lipids (137-139) to peptides $(140,141)$, proteins $(142,143)$, and protein complexes (144). In MALDI-MSI, IMS helped to spatially localize and structurally identify acylcarnitines and a variety of lipid species present in breast tumour xenograft models (137). In DESI, ion mobility demonstrated its use in studying fragile species like gangliosides thereby generating complementary information to MALDI-MSI (138). Recently, FAIMS (field asymmetric waveform ion mobility) has been introduced and coupled with DESI-MSI; it has been demonstrated to increase sensitivity and signal to noise of gangliosides and cardiolipins (145).

\section{Identification strategies for metabolites, peptides, and proteins}

The technical advances mentioned in the section before, especially the availability of high mass resolution and additional separation techniques such as IMS, have also helped improving the ability to determine the identity (ID) of detected $m / z$ species. This is of key importance in MS imaging when investigating potential new biomarkers, understanding the biology and chemistry of a diseased system, or confirming the identity of targeted compounds. In MSI, the identification of certain compounds is either performed by MS/MS experiments directly from tissue or/and by matching the measured $m / z$ value to a database. On-tissue procedures face the challenge of small amount of analytes present in an imaging pixel (in a $50 \mu \mathrm{m}$ pixel there are about 25 cells). Ionization procedures that could increase the ion yield per pixel would be valuable. Using a second laser to initiate a second ionization 
wave in the gas phase, Soltwisch et al. increased ion yields by up to two orders of magnitude (146). But this was restricted to lipids so far. The database approach makes use of either public databases (e.g. METLIN or MSiMass list) or private databases based on a set of compounds which have been previously determined to be present in that sample by separate proteomics/metabolomics experiments from tissue extracts (147). Overall, there are different identification procedures, which depend on the compounds of interest:

Intact proteins. The majority of intact protein MSI experiments are performed on linear time-of-flight (TOF) mass analysers, which do not return structural information for direct on-tissue identification. Consequently, one way of identifying proteins has therefore been to first extract all protein content from the tissue, then separate the proteins by HPLC fractionation, and perform either top-down or bottom-up analysis on the purified fractions containing the masses of interest $(26,148)$. Also, while TOF-MS offers the benefits of a wide mass range and high throughput, it falls short in mass resolution in the higher mass range. FT-MS provides a significantly higher mass accuracy than TOF analysers. Spraggins et al. have used FT-ICR MS to image proteins up to $12 \mathrm{kDa}$ and enable their identification based on correlating the accurate protein signals ( $<5 \mathrm{ppm}$ ) with top-down proteomics data (149). The results of such experiments are very valuable input for public protein MSI databases, such as MSiMass list (150).

Peptides. The database lookup approach can be applied to peptides as well, where highly accurate masses can be correlated to the results of parallel bottom-up proteomics experiments (109). This must, however, involve on-tissue digestion in the MSI sample preparation. Heijs et al. have optimized the use of several MALDI-compatible proteases, which enabled to assign 630 peptides, corresponding to 280 proteins, to mass peaks found in the FT-MSI data sets (109). In the mass matching approach, the confidence in identification is directly dependent on the mass accuracy of the MSI experiment. Schober et al. have determined the necessary mass tolerance to be less than $3 \mathrm{ppm}$ (151). But in contrast to proteins, peptides can also be identified directly from tissue by MS/MS experiments. The use of TOF/TOF analysers for on-tissue identification has also been demonstrated, where the first TOF is used to select a precursor ion for fragmentation and the second TOF is used for the rapid and parallel analysis of fragments allowing identification of the isolated compound of interest (152-154). The identification from tissue is challenging due to the lack of chromatographic separation in an imaging experiment. Adding an additional separation step after surface sampling, either based on gas-phase ion mobility or liquid-phase chromatography (LC), can provide improved selectivity and hence confidence in analyte identification. To illustrate, LESA-MS combined with bottom-up proteomics provides a way to increase the number of identifications to around 500 proteins, but comes at the price of lower spatial resolution (69).

Small metabolites and lipids. Due to lack of comprehensive MALDI and DESI MS/MS databases and search algorithms for lipids and small metabolites, high mass accuracy provides an important indication of identity. A common approach is to first match the measured molecular weight with a small mass tolerance to a public database, such as HMDB (http://www.hmdb.ca/), METLIN (https://metlin.scripps.edu), or lipid maps (http://www.lipidmaps.org/), and then check if the observed fragmentation spectra are in line with the structure of the proposed match (155). Final confirmation can be obtained by 
comparing the fragmentation pattern with one from a corresponding standard compound (118).

\section{Three-dimensional MSI}

The reconstruction of analyte abundances in an entire three-dimensional (3D) volume allows for delivering contextual information to $2 \mathrm{D}$ images and reducing the possibility of neglecting small anatomical structures. 3D MSI data can be created in two ways: i) by sequential acquisition and registration of multiple 2D tissue sections followed by combination into a single 3D representation achievable with MALDI (156-158) or DESI (159), or ii) by depth profiling using secondary ion mass spectrometry (160-164).

For the first approach, the accurate coregistration of a stack of consecutive 2D MSI images is the most critical and difficult step. In addition, comparison with other modalities (e.g. MRI (165)) can be required. Chughtai et al. have developed a coregistration technique, which consists of using fiducial markers with optical and molecular properties. These markers allowed on the one hand the accurate coregistration of multimodal data from MSI with histological and fluorescent microscopic images. On the other hand, it also allowed coregistering of a set of serial tissue sections to reconstruct 3D MSI images of a breast cancer sample (166). Also, recently, Patterson et al. described how open-source software can be used to obtain high-quality visualizations and multivariate segmentations from 3D MSI data (167). By analysing atherosclerotic plaques, they also demonstrated the added value of 3D MSI for the investigation of samples where the volumetric spatial organization is important. Furthermore, 3D MSI has been used successfully in the field of microbiology to characterize microbial metabolic exchange (157) and also for 3D single cell imaging $(156,160,164)$.

\section{Advances in bioinformatics}

The success of a MSI study is strongly dependent on downstream data analysis capabilities. Clinical MSI usually involves the analysis of dozens to hundreds of samples to achieve the required statistical power. With the advent of high mass and high spatial resolution data sets, and therefore individual data set sizes in the gigabyte range, the processing and handling of data in a clinical MSI study has become computationally demanding. While vendors are incorporating on-the-fly data reduction and processing methods during acquisition, software developers have come up with intuitive and efficient MSI software packages to facilitate the analysis of clinical MSI data. Commercial software packages, such as Multimaging (Imabiotech, France) or SCiLS (Bruker Daltonics, Germany), can handle terabyte-sized, multi-sample datasets and include many statistical tools for biomarker discovery. Open-source software is also available, such as MSiReader (168), Cardinal (169), Biomap (170), msiQuant (171), or SpectralAnalysis (172), but less focused on the simultaneous analysis of several samples.

A special interest of researchers has also been to further develop data analysis techniques, which account for the very special nature of MSI data, i.e. multivariate data in a spatial context. Van de Plas et al., for example, correlated the multivariate patterns of low resolution MSI image with the histological entities from a higher resolution optical image in 
order to create super-resolution MSI images at the resolution of the optical image (173). In this way they were able to increase the resolution of an MSI image by a factor of ten. The spatial context of MSI data was also exploited in another study. So far, classical statistical tests, such as a t-test, within a sample were not valid due to the spatial dependence of the single spectra. Using conditional autoregressive models, which account for the spatial autocorrelation of mass signals, within-sample statistical comparisons could be performed. This is useful, for example, in determining significant differences in drug distributions in a tissue (174).

Clustering is a powerful tool in MSI and has been demonstrated to be capable of differentiating tumour types, visualizing intratumour heterogeneity, and segmenting anatomical structures (175). Also, clustering algorithms have been improved by incorporating the spatial information of the data (176).

Likewise, the project MetaSpace uses the spatial information of the detected isotopic patterns to increase the confidence of metabolite identification (177). The MetaSpace project is an open-data repository where every active user can see the data of other users. The open design also enables the sharing of knowledge represented by manual annotations of molecules.

\section{Sharing data and knowledge}

Sharing data and knowledge is becoming more important, in general, in MSI. This is particularly critical for clinical MSI studies, where different scientific disciplines, represented by clinicians, biologists, chemists, and data analysts, have to work side by side. Therefore, platforms to share data and knowledge (and the capability to process and analyse data together) are becoming vital for the success of projects.

Building blocks for these platforms are common standards and open data repositories. Introduced in 2012, imzML has become the de facto data exchange format in MSI, which is now also supported by most instrument vendors (178). Data repositories for MSI data followed, such as the open ProteomeXchange platform (179) and MetaSpace, which allows online viewing of MSI datasets (177). The commercial SCiLS lab cloud (scils-cloud.de) goes a step further, allowing all collaborators including clinicians to contribute their knowledge on the same data online, similar in function to a Google document.

Other building blocks for sharing knowledge are common standards for reporting and guidelines for setting up and interpreting the results from clinical MSI experiments. Steps towards this have been taken by proposing guidelines for reporting MSI data and results based on the MIAPE (minimum information about a proteomics experiment) standard (180). Finally, Lou et al. have reported a comprehensive manual for a successful biomarker discovery in histologically complex tumour tissues. The aspects ranged from tissue preparation and data acquisition to data quality control, histology-defined data selection, data processing, and statistical analysis (181). 


\section{Highlights of clinical research performed with MSI}

Historically, clinical MSI was mainly represented by MALDI-MSI, orientated towards protein biomarker discovery, given the fact that results can be validated by existing techniques such as LC-MS/MS, SDS-PAGE, or immunohistochemistry. With the advent of ambient ionization methods, many studies have used DESI-MSI for clinical tissue classification based on lipid patterns. These two MSI-techniques have already proven to provide relevant information in several clinical research areas. Starting with accomplishments in oncology, which are summarized in figure $\mathbf{4}$ and where solid pillars have been built towards the use of MSI for diagnostics or prognostics purposes. This section reviews major past and present highlights of clinical MSI in various research areas such as neuropsychiatry and neurology, nephrology, ophthalmology, and finally rheumatology.

\section{MSI in oncology}

Biomarker discovery. Biomarker discovery has the aim to identify patterns or single signals of biomolecules that can stratify patients according to their diagnosis, disease state (staging), and prognosis to enable a more personalized therapy. In 2003, Yanagisawa et al. were the first to report the successful use of MALDI-MS tissue profiling combined with classprediction models based on differentially expressed proteins to accurately determine lung cancer histology, distinguish primary tumours from metastases, classify nodal involvement, and predict the prognosis of patients (26). This study was not an imaging study, but was an MSI landmark paper as it gave evidence of the potential of biomedically relevant information that can be obtained when interrogating tissue directly by MALDI-MS. The same group showed a few years later that MALDI-MS imaging was also able to obtain similar information from archived tissues where peptide patterns were found in 112 biopsies from lung-tumour patients to discriminate adenocarcinoma from squamous cell carcinoma (128). In the following years many oncological studies have exploited the potential of MSI to find biomarkers for diagnosis, prognosis, or therapy response prediction.

Diagnosis. Some of the first cancer MSI studies were interested in finding diagnostic markers, where the tumour patterns were compared to the healthy tissue correlate. One of the first studies in 2007 used MALDI-MSI to find the protein fragment Reg alpha as potential biomarker for ovarian cancer, which has been confirmed in a second study by immunohistochemistry (IHC) on 179 tissue samples (182,183). In 2009, Cazares et al. found $\mathrm{m} / \mathrm{z} 4355$ to accurately discriminate cancer from uninvolved prostate tissue. The molecule was identified by on-tissue MALDI tandem mass spectrometry as a fragment of mitogenactivated protein kinase/extracellular signal-regulated kinase kinase kinase 2 (MEKK2). The knowledge of the identity enabled to investigate and confirm the discriminatory power of MEKK2 orthogonally by IHC (184).

Classify preneoplastic lesions. The correct classification of preneoplastic lesions during screening or surveillance is crucial for initiating a curative treatment. For example, patients with intestinal metaplasia in the oesophagus are at higher risk for developing cancer. Elsner et al. have shown that the histological specificity of MSI provides a powerful tool to distinguish Barrett's adenocarcinoma from its premalignant stages based on several differentially expressed proteins (185). Another group at risk are patients with cirrhosis since they might develop liver cancer (HCC). Laouirem et al. investigated the mechanisms 
associated with the transition from cirrhosis to HCC and found increased ubiquitin truncation catalysed by an elevated de novo expression of KLK6 along cancerogenesis (186). Similarly, Rebours et al. identified ubiquitin and thymosin-beta 4 as markers of malignant intraductal pancreatic mucinous neoplasms, which are precancerous cystic lesions (187). The robustness of the results was further corroborated in larger cohorts using TMAs and endoscopic ultrasound fine-needle aspiration.

Staging. Once the diagnosis of the presence of cancer has been made, the disease stage has to be determined. The first examples of MSI for tumour staging go beyond classical histopathological examination where MALDI-MSI was used for the classification of HER2 status in breast and gastric cancer tissues $(148,188)$. Since both routine immunohistochemistry and fluorescence in situ hybridization suffer from subjective interpretation, automated signature-based classification opens new options for an objective HER2 status annotation. The results of Rauser and co-workers also demonstrated that MALDI-MSI can reveal biologically significant molecular details from tissues which are not limited to traditional high-abundance proteins (148). While the HER2 status is an important determinant for therapy decision-making, the presence of lymph node metastasis is the strongest determinant for patient prognosis. Hence its determination is of utmost importance. In colon cancer tissues, protein expression patterns as detected by MALDI-MSI were able to distinguish between patients with and without metastasis formation (189).

a)

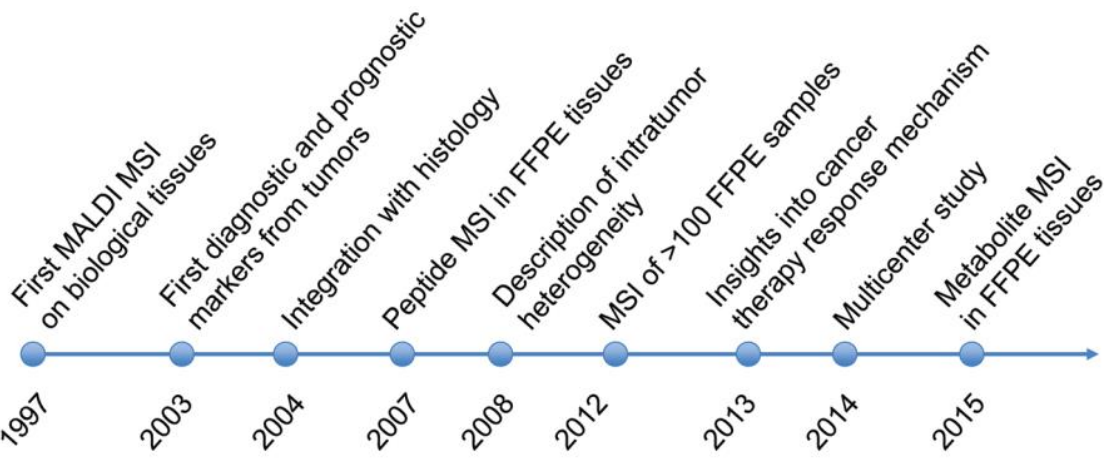

b)

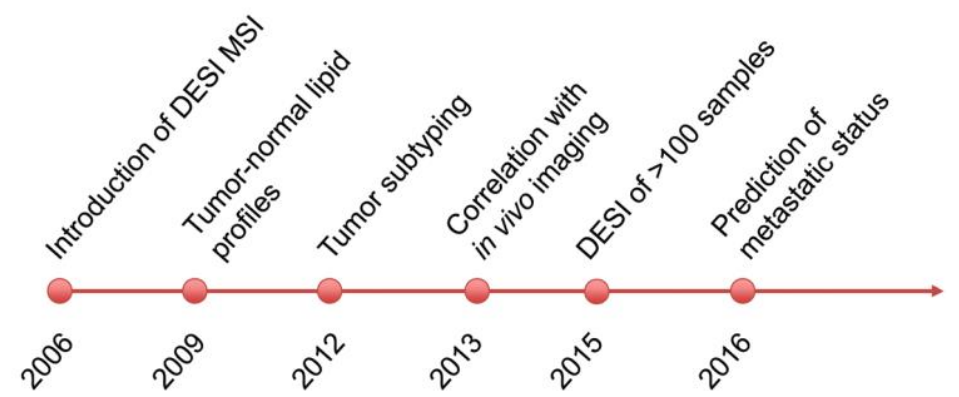

Figure 4. Highlights and breakthroughs of MALDI- and DESI-MSI in oncology. (a) Highlights in oncology for MALDIMSI in chronological order and their corresponding references $(25,26,48,83,107,118,190-192)$. (b) Highlights in oncology for DESI-MSI in chronological order and their corresponding references (193-197). 
Prognosis. While patients may share the same diagnosis and tumour stage, they might differ in their prognosis after therapy. MALDI-MSI has been used in many studies to find markers or signatures that can stratify patients according to their survival time after treatment. Several protein signatures were found associated indicative of unfavourable overall or disease-free survival in intestinal-type gastric cancer (198), colon cancer (199), sarcomas (200), oesophageal cancer (185), and lymph node positive breast cancer (201). With respect to tumour recurrence, Hardesty et al. were the first to show that protein signatures can be found that can predict relapse in metastatic melanomas (202). In another study, modified forms of histone $\mathrm{H} 4$ were found indicative of microvascular invasion in hepatocellular carcinomas (HCC) - a major risk factor in postoperative tumour recurrence and mortality in HCC, usually missed in routine examination from liver biopsy (203).

Response prediction. Many patients undergo chemotherapeutic treatments, such as neoadjuvant to reduce tumour size before the surgery or adjuvant to reduce cancer recurrence probability after surgery. However, this is a stressful procedure for the patient, which should be avoided for patients who are not responsive to chemotherapeutic treatment. For this reason, there is a strong need for accurate chemotherapy response markers. In breast cancer, MALDI-MS tissue profiling and imaging enabled identification of proteins in pre-treatment biopsies that predict response to neoadjuvant taxane-based therapy (204). The knowledge of molecules that are associated with therapy response might also allow insight into the therapy resistance mechanisms of the tumour. This was shown in a study on oesophageal adenocarcinomas where a protein signature was first found to be correlated with the chemosensitivity to fluorouracil and cisplatin administration (192). This finding initiated subsequent in vitro experiments, which showed that the chemosensitivity to the treatment was correlated to pre-existing defects in mitochondrial respiratory chain complexes of cancer cells, caused by the loss of specific cytochrome $\mathrm{C}$ oxidase subunits.

Molecular histology. Tissue diagnostics can be challenging due to the presence of confounding factors such as inflammation or the lack of a minimum level of differentiation of tumour cells. To illustrate, high-grade sarcomas are diagnostically challenging because of their numerous subtypes that possess overlapping morphology. MALDI-MSI was able to differentiate high-grade osteosarcomas, leiomyosarcomas, and myxofibrosarcomas, and to predict the precursor (and therefore appropriate therapy) of undifferentiated pleomorphic sarcomas $(181,200)$. Another pathological challenge is the distinction between atypical Spitzoid neoplasms and melanomas. Based on histology-guided MALDI-MS profiling of the tumour areas, a peptide signature, including signals from actin and vimentin, was able to distinguish Spitz nevi from poor prognosis Spitzoid malignant melanomas with high accuracy (191). This study remains unique in MSI clinical applications, since the signature has proven so robust that the study was extended to 11 centres (205).

Intra-tumour heterogeneity. Tumours are very complex tissue systems and intra-tumour heterogeneity can be perceived as the sum of sub-histological segments. These segments may react differently to therapeutic interventions (e.g. chemo- and radiotherapy) and therefore show characteristic phenotypic behaviour regarding aggressiveness and metastasis formation; thus contributing to the overall clinical disease phenotype. It was demonstrated early on that MSI constitutes a unique tool for the investigation of intratumour heterogeneity (190). But only recently MALDI-MSI investigations have been extended to the characterization of intratumour heterogeneity (206). In that study, 
unsupervised clustering revealed those tumour subpopulations in gastric and breast cancer patients that are associated with different clinical phenotypes such as overall survival or the presence of loco-regional metastases, respectively. This will allow their more in-depth molecular characterization for deriving new tumour subpopulation-tailored therapeutic strategies.

Lipid and small metabolite markers. If most of the applications reviewed so far rely on protein signatures (fig. 4 a), it is noteworthy that -especially with the instruction of DESI in 2006- an increasing number of investigations focus on lipid and metabolic MSI to characterize tumours and surrounding tissues (fig. 4b). It has become evident already that these signatures can harbour rich information for oncologic research similar to that of proteomics. The prospective collection of samples for metabolic profiling is beneficial as the tissue conservation procedure can strongly influence metabolic activity/degradation (207). In 2015, for instance, Guenther et al. conducted a prospective DESI-MSI study to fingerprint 126 human breast cancer biopsies (196). They found substantial differences in fatty acid and phospholipid composition between tumour and healthy glandular tissue and between tumour-associated stroma and normal stromal tissue. Moreover, patterns were correlated with tumour grade and hormone receptor status. The ability of lipid-based DESI-MSI patterns to predict a molecular status of therapeutic relevance was corroborated in a study on colorectal adenocarcinoma, where a multivariate model showed proper separation of the KRAS mutation status in primary tumours and liver metastases (208). Eberlin et al. further exploited the ability of DESI-MSI for tumour subtyping in 36 human gliomas. Classifiers based on lipid data showed a high predictive power for subtyping gliomas into oligodendroglioma, astrocytoma or oligoastrocytoma, and assessing the histologic grade and tumour cell concentration features. Interestingly, DESI-MSI was also able to distinguish samples characterized by the presence of a specific cell type, namely gemistocytic cells which presented a distinctive lipid profile with abundant fatty acid and fatty acid dimer signals (195). In another study, DESI-MSI was used to investigate the lipid composition on human oncocytic, non-oncocytic thyroid tumours, and normal thyroid tissues $(n=45)$. Oncocytic tumours are characterized by an excessive accumulation of mitochondria. The results revealed high abundance of many oxidized cardiolipins in oncocytic tumours. For the first time, an organelle-specific biomolecular lipid subclass has been associated with pathological cell subtypes as a cluster for cancer classification on human samples (64).

MALDI-MSI was also successfully applied for the metabolic profiling of cancer tissues. In the study of Patterson et al., lipid data was obtained from neoadjuvantly-treated colorectal cancer liver metastases (209). The signatures were found to accurately compare with histopathologically important features, including different types of necrosis, to assess therapy response objectively. For confirmation, these MSI response scores were also found to correlate with the survival probability of the patients. Shortly after, Lou et al. also ascertained the prognostic value of several small molecules in a cohort of frozen sarcomas (210). Buck et al. showed that even in FFPE tissues clinically relevant information can be conserved in the form of metabolites. They found signatures for the diagnostically challenging separation of oncocytomas from chromophobe renal cell carcinomas, and deoxy sugar acids with sulphate esters for prognostication in patients with oesophageal cancer (fig. 5b) (118). 
Characterization of cancer margins to support intraoperative diagnostics. The emergence of ambient ionization techniques and high-speed analyses (fig. $\mathbf{2 f}$ and fig. 3a) has placed MSI in a very competitive position to characterize cancer tissues in a very short time compatible with diagnostic routine. Consequently, MSI has the potential to support decision-making based on frozen sections in a perioperative setting. In cancer surgery, the evaluation of resection margins for residual tumour cells is considered of utmost importance to avoid a later recurrence or metastasis (66). In 2010 and 2014, MALDI-MSI investigations on colorectal and renal tumour margins revealed differences between tumour-adjacent healthy tissue compared to tumour-remote healthy tissue on both a metabolic and proteomic level $(211,212)$, showing the necessity to complement classical histopathological inspection of the tumour margins by a molecular technique. Based on these findings, several DESI-MSI studies followed that made use of these tumour-specific patterns to assess tumour margins (fig. 5c).

The majority of meningiomas are benign tumours, but their resection quality is limited by a proper assessment of the invasion extent of the dura matter by meningioma cells. DESI-MSI could not only distinguish meningioma from dura matter to precisely define the surgical margins, but also determine the meningioma subtype (fibroblastic or meningiothelilal) and its NF2 genetic aberration status (213). The feasibility of tumour margin assessment by classifying cancerous and not cancerous tissues based on molecular signatures in perioperative settings by DESI-MSI was also demonstrated for breast (214), gastric (215), and pancreatic cancer (216).

In addition, DESI can also be used to trace known administered or endogenous markers. With respect to the first, Tata et al. used DESI-MSI to detect the contrast agent Gadoteridol in order to image the tumour margins and vasculature in breast cancer xenografts (217). An example for the successful visualization of specific endogenous tumour markers by DESIMSI is 2-hydroxybutyrate, which is generated by isocitrate deshydrogenase 1-mutant gliomas only (217). In the same study, the measured data was also fused with an MRI reconstruction of the tumour to improve decision-making. This example shows how ambient MSI techniques can be integrated with other imaging modalities with the ultimate goal to improve intraoperative decisions.

Another important factor for cancer resection is the dispersion of the tumour to the lymphatic system. During surgery the regional cancer dissemination from its primary site can be monitored by a histopathological ex vivo evaluation of sentinel lymph nodes. The presence of metastases in these lymph nodes determines the extent of the clearance of the remaining regional lymph nodes. In two DESI-MSI studies on gastric and oesophageal cancer, Abbassi-Ghadi et al. found lipid patterns for the accurate recognition of affected lymph nodes $(197,218)$. While these lipid signatures achieved similar accuracies $(100 \%$ and 97.2\% positive and negative predictive values, respectively) as the gold standard immunohistochemistry (197), DESI-MSI is much faster and hence could give feedback during the time course of the surgical intervention about the detection of lymph node micrometastases bigger than $200 \mu \mathrm{m}$.

Touch spray and in vivo MS. MSI affiliated techniques are also garnering strong interest for surgical diagnostics because they can quickly and accurately classify macroscopic regions on biological samples by exploiting the specificity of local molecular signatures. One of those techniques for local profiling of tissues is touch spray, where a tiny sample is taken with a 
needle from tissue and its compounds are exposed to spray ionization in the needle (219). Two extensive studies on prostate $(n=170)$ and renal cell cancer $(n=29)$ have investigated the potential of touch spray to distinguish tumour from tumour-free tissues $(220,221)$. In both studies touch spray achieved comparable accuracies (>90\%) to DESI-MSI, while providing faster analysis times. This calls into question the need to image the tumour margin.

Another interesting technology for surgery is rapid evaporative ionization mass spectrometry (REIMS), which allows near real-time characterization of human tissue in vivo by analysing the smoke released during electrosurgical dissection of the tissue (222). Being compatible with current electrosurgical procedures, REIMS has demonstrated great potential for in situ, in vivo and real-time tissue evaluation to improve intraoperative decision-making. Recently, REIMS has been adapted to endoscopic interventions during gastroscopy or colonoscopy, where tissue-specific molecular fingerprints allowed differentiation between healthy mucosa, adenomatous polyps, and cancerous tissue $(223,224)$. In colon cancer, REIMS was also able to determine the degree of a tumour's differentiation, lymphovascular invasion, extramural vascular invasion, and lymph node micrometastases (223). Based on this first successful evidence, comparable techniques are being developed, such as SpiderMass, which promises equal readout of biomedically relevant information while reducing the invasive condition (225).

Cross organ studies. Pirro et al. compared DESI-MSI data from both cancerous and corresponding healthy tissue from different human organs including bladder, kidney (papillary and clear renal cell carcinoma), germ cell, and prostate. This has led both to the identification of cross-tissue cancer markers such as the lipids $\mathrm{PI}(18: 0 / 20: 4)$ and PS(18:0/18:1) as well as to the identification of tissue-specific markers such as cholesterol sulfate for prostate cancer and seminolipid for germ tissue (226). Meding et al. have further demonstrated the usefulness of cross organ molecular profiling by classifying cancers of unknown primary (CUP) (227). CUP is a clinically challenging diagnosis and a better therapy can be applied if the origin of a metastatic tumour is known. Therefore, proteomic patterns were obtained from six primary cancer types by MALDI-MSI and a classifier was created to distinguish primary tumours. The potential of this classifier for CUP origin determination was successfully demonstrated on colon cancer liver metastasis.

Preclinical models of diseases and drug imaging. While MS molecular profiling of tissues linked with histopathology can be a valuable tool for cancer biomarker discoveries on human samples, MSI is also applied in preclinical research studies where animal models are used in a controlled setting to better understand the mechanisms of pathological disorders.

MYC is one of the most common oncogenes implicated in human cancerogenesis and its relation to the presence of certain lipids is unknown. Therefore, MYC-induced lymphomas in transgenic mice were investigated by DESI-MSI and a MYC-specific pattern of complex phospholipids was observed. Moreover, this pattern of MYC-induced mouse lymphomas was found to be similar in human lymphomas with high MYC expression (228). This study shows that in some cases MSI-derived patterns may be translatable from preclinical animal studies to the classification of human tissues. In another study, Grüner et al. used MALDIMSI to characterize $250 \mu \mathrm{m}$ small precursor lesions of pancreatic ductal adenocarcinoma (PDAC) in genetically engineered mouse models. The analysis of these lesions identified the two proteins ALB1 and TMSB4X as being highly upregulated in the preneoplastic stage 
compared to a healthy exocrine pancreas (229). The validation also showed that TMSB4X was found to be significantly increased in sera of mice with lesions, and that ALB1 upregulation was accompanied by an increased expression of liver-restricted genes. The same researchers also used MALDI-MSI to study the delivery, spatial distribution, and effect of the anti-cancer drug erlotinib on this PDAC mouse model (230).

a)

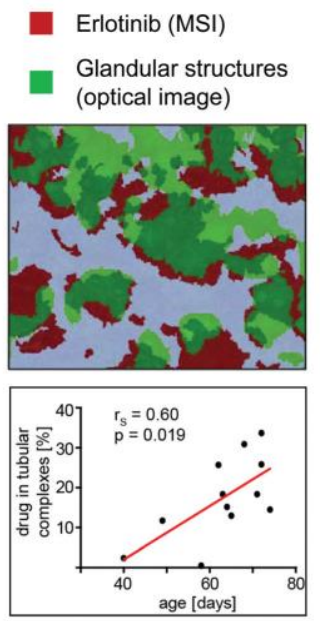

b)

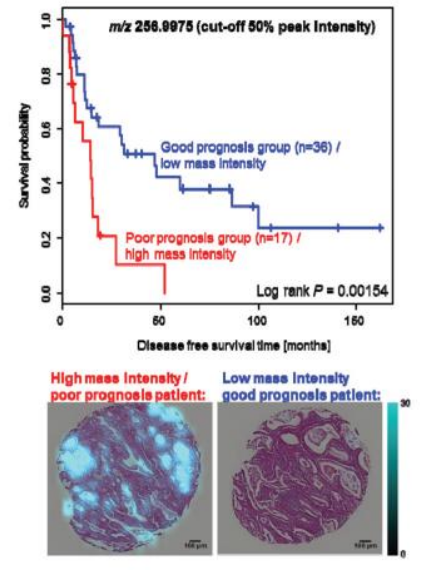

c)
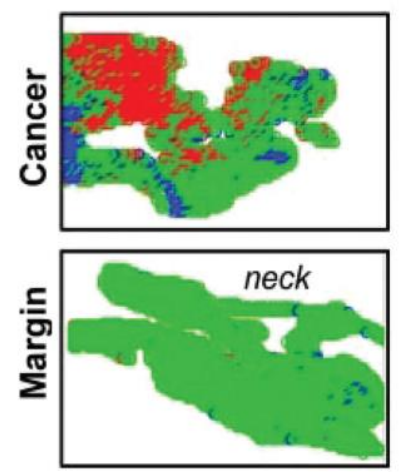

- cancer =normal glands - normal stroma

Clinical research

\section{Pre-clinical research}

\section{Translational research}

Figure 5. Range of translational research areas covered by MSI studies. We refer to the definition of "translational research" as proposed by Rubio et al. where translational research integrates basic, patient-oriented, and population-based research, with the long-term aim of improving public health(231). MSI can be found across the whole span of translational research. (a) Grüner et al. recently published a pre-clinical study on imaging erlotinib in transgenic mice. This study demonstrated the importance of spatial MS, since the overall survival was only correlated with levels of erlotinib (lower panel) when the drug was localized in the glandular structures of the pancreas (upper panel). Adapted with permission from Grüner et al. (230) Copyright (2016) American Association for Cancer Research. (b) Buck et al. conducted a biomarker discovery study in formalin-fixed paraffin-embedded tissue tissues and found a metabolite (a deoxy sugar acid with sulphate esters), which is located in mucinous areas, with prognostic value for patients with oesophageal cancer. Reprinted with permission from Buck et al. (118) Copyright (2015) John Wiley and Sons. (c) The type of clinical MSI research with the highest translational character is the perioperative assessment of tumour margins. Eberlin et al. convincingly demonstrated in several studies the accuracy of spatially resolved lipid patterns for detecting tumour residues in surgical specimens, here shown for pancreatic cancer, and its potential superiority, compared to classical histological evaluation, to predict recurrence (216).

For the pharmaceutical industry, compared to autoradiography and LC-ESI-MS/MS on tissue homogenates, MSI represents an unrivalled tool to monitor without labels not only the drug distribution, but also the products of its metabolisation in a tissue or a whole small animal $(120,121)$. Even if MSI faces some limitations in the detection and quantification of molecular classes, the endogenous molecular changes linked to the drug and its metabolite distributions may lead toxicological pathology to a new level. For example, a TOF-SIMS study on benzalkonium chloride, a commonly used preservative in eye drops and reported ocular 
surface toxic, revealed its penetration deep into ocular structures, with potential deleterious effects (232). Likewise a MALDI-MSI nephrotoxicity study on the effects of the anti-cancer drug dabrafenib reported accumulation of the drug's carboxylic acid metabolite in regions of tubular deposits, but not of the drug itself (233). This kind of information can be valuable for drug efficiency tests with respect to tissue affinity of the drug and its metabolites. Until now, most of the MSI studies performed so far have been oriented more towards imaging the drug or its metabolite within their histopathological context. As mentioned before, Grüner et al. conducted an MSI study on erlotinib, which was the first drug imaging study to investigate the effect of a drug's distribution on the survival time of mice (230). The study also shows the importance of the integration of histological information into the analysis, since the overall survival was only found to correlate with the erlotinib levels found in epithelial structures (fig. 5a). Similarly, AIMS (affinity imaging MS) targets screening for new drug candidates by analysing the specific affinity of compounds to particular tissue regions after in vitro incubation with a mixture of small molecules (234).

\section{MSI in non-cancer clinical research}

Besides in cancer, MSI has been applied to many other clinical research questions where diseased tissue exhibits a certain spatial complexity.

MSI in neurological diseases. Because of the anatomical and molecular complexity of the central nervous system, MSI has gained wide interest in the investigation of brain-related psychiatric, neurological, and neurodegenerative diseases such as amyotrophic lateral sclerosis (235), Alzheimer's disease (236,237), migraine (238), neurotraumas (239), Parkinson's disease (240-242), schizophrenia (243), Hunter's disease (244), and brain ischemia (245). Two reviews have summarized the application of MSI in neurological diseases $(246,247)$.

MSI in cardiovascular diseases. Atherosclerosis is one of the main interests in cardiovascular research, and $\mathrm{MSI}$ has given the opportunity to study the molecular content of atherosclerotic plaque and the arterial layers in order to shed light into the molecular processes during plaque formation. It has been shown that high spatial resolution (248) and 3D imaging (167) of the plaque is beneficial for the study of the atherosclerotic plaque. Martin-Lorenzo et al. recently reviewed the current status of atherosclerosis investigations by MSI (249). Also cardiac tissue has been analysed such as after myocardial infarction (250) or aortic stenosis, the most common valve disease (251). Angel et al. have reviewed MSI of cardiac tissue (252).

MSI in rheumatology. Investigations into rheumatic disorders by MSI have recently been summarized (253). One of the most studied pathologies in rheumatology is osteoarthritis (OA). The pathogenesis involves a progressive degradation of the extracellular matrix of the cartilage, which is formed by chondrocytes, and a remodelling of the synovial membrane and subchondral bone. MSI has been used to study OA cartilage, synovial membrane, and chondrocytes on a metabolic, lipid, protein, and N-glycan level (254). In this way, markers have been found that are able to distinguish healthy from OA tissues $(255,256)$, as well as markers for chondrogenic differentiation $(257,258)$ and aging of cartilage $(259)$.

MSI in nephrology. In nephropathology, MSI has been mainly applied to glomerulonephritis and amyloidosis. The former is a group of kidney diseases, which is characterized by the 
inflammation or scarring of the glomeruli. Magni et al. found in two MALDI-MSI studies several signatures that could distinguish between healthy and diseased glomeruli and between different glomerulonephritis diseases $(260,261)$. In contrast, amyloidosis is a disease not exclusively related to the kidney. It is a heterogeneous group of protein misfolding diseases characterized by deposition of amyloid proteins, and diagnosed at the histopathological level with Congo red staining and electron microscopy. Amyloids are fibrillar aggregates of polypeptides in a cross-B-sheet structure involved in the pathogenesis of amyloidosis. MSI seems promising for subtyping amyloidosis with the aim of revealing amyloid-associated proteins that may provide insights into the pathogenic process of amyloid formation. Casadonte et al. used MALDI-MSI to find peptides in Congo red-positive areas of the kidney (262). One of those was identified as vitronectin, which was also found by another MALDI-MSI study and validated using immunohistochemistry across 175 patients with diverse types of amyloidosis and affected tissues (263).

MSI in ophthalmology. MSI investigations in ophthalmology were recently summarized (264). All of the studies show the importance of obtaining spatially defined molecular information from ocular tissues to better characterise several vision-threatening diseases, including age-related macular degeneration $(265,266)$, Stargardt disease $(267)$, glaucoma (268), and cataracts (269). But MSI has also been used to study the biology of the healthy eye, such as the metabolic changes associated to light- and dark-treated retina (270).

Characterization of the wound healing process. Wound healing appears to be a particularly interesting pathological process to be explored locally by using MSI. Taverna et al. studied cutaneous wound healing in human frozen skin biopsies of decubitus ulcers with different stages and responses of healing (271). The spatial character of MALDI-MSI allowed the extraction of proteomic content from the wound bed, adjacent dermis, and hypertrophic epidermis. A signature mainly composed of calcium binding proteins (S-100 molecules) was able to delineate wounds that favourably responded to therapeutic interventions versus those that remain stagnant or intractable in their healing status (271). The same authors further investigated the molecular changes during burn wound healing, i.e. from the acute period (days after trauma) to the later hypertrophic scar (months after trauma) (272). This histology-directed tissue analysis found that the expression of several inflammatory proteins correlated to the wound healing process, which could be used to predict whether human burn victims are at risk of disfiguring hypertrophic scar formation.

Immunology. Finally, Holzlechner et al. demonstrated that MALDI-MSI can also be used for an in situ molecular characterization of immune cells in human colon tissue, which is by nature strongly infiltrated with immune cells (84). In this example, several proteinaceous markers were identified that co-localized with CD3+ T-lymphocytes and CD206+ macrophages. Because different types of immune cells can be visualized and molecularly assessed in their natural environment, further study of the cross talk between lymphocytes and their tissue microenvironment will be enabled. In a previous study of autoimmune liver diseases, it was already shown that protein patterns of inflammatory infiltrates were different from bile ducts and hepatocytes (273). Interestingly, the authors could not find any proteins that could distinguish with certainty between the three major forms of autoimmune liver. 


\section{Present challenges in translational research performed with MSI}

As shown in the previous section, MSI contributes novel and valuable information on many biomedical research questions. But all of these applications of MSI have also revealed specific limitations with respect to the requirements of clinical research which has two main interests: first, improving diagnostic certainty through biomarker discovery, and second, improving understanding of the biological mechanisms of the studied pathology with the final aim of being able to develop novel therapies.

Biomarker discovery is all about reproducibility and accuracy, i.e. to find hidden markers that most exactly stratify patients according to a certain clinical feature in different labs with the same precision. Similar to other omics techniques, MSI produces high-dimensional datasets on a sample number, which is usually orders of magnitude lower than the number of detected features. In this scenario, the resulting data will suffer from the so-called "curse of dimensionality", which can lead to potentially false-positive discoveries and to overfitting of multivariate classifiers. The final consequence of both is non-reproducibility (274). A suitable validation of the results can overcome this problem (275). There are different levels of validation, each providing a different level of evidence for the original finding. Validation approaches range from technical replicates, the use of independent methods or sample cohorts to multicentre studies, which provide the highest level of evidence as it removes biases based on laboratory conditions (fig. 6) (276).

Many MSI studies have validated their results in different ways. If protocols and antibodies are available, protein biomarker findings have been mostly validated using immunohistochemistry as an independent technique (277). However, when it comes to multivariate patterns, the classificatory combination of single signals is only replicable by the same methodology. In this case, either a computational solution can be employed (e.g. by using cross-validation) (277), or the classifier must be tested on a second independent sample cohort (148). But the ultimate step from the clinical exploratory phase to the phase of clinical assay validation is a multicentre study.

So far, only one MSI biomarker publication has been based on a multicentre study with the aim of identifying markers for the activation of tumour stroma in breast cancer (83). There are some conclusions that can be drawn from this study. First, three out of four univariate protein signals were found reproducible between the two centres. This indicates high specificity in the MSI results, which might be attributed to the histological specificity of the MSI approach. Second, the multivariate classifier's accuracy (a non-significant drop from $100 \%$ in the first to $81 \%$ in the second centre) exhibited the highest difference between centres, which is in line with the experience from gene-expression studies and hence suggests a more rigorous validation over more centres or the involvement of more samples (278). And last, the biological effect was robust enough despite minor differences in the experimental protocols between centres and composition of the patient cohorts. However, future multi-centre studies should standardize their protocols in order to become more reproducible for clinically relevant questions based on smaller biochemical changes at danger to be masked by inter-centre variance.

To overcome inter-centre variance, MSI experiments can also be centralized for the analysis of samples from difference centres. This has been done for a study on over 250 atypical Spitzoid neoplasms, which was able to find clinical associations of MSI data across centres 
from 11 countries and 11 US institutions (205). This is remarkable as the study was performed not only on FFPE specimens but also on full sections of those samples. To put this into context, most MSI biomarker studies reporting results that involve FFPE samples in this magnitude have been performed on tissue microarrays to reduce the inter-sample technical variance caused by FFPE sample preparation, which is chemically intense due to antigen retrieval and in situ enzymatic digestion. Measures of reproducibility have therefore been proposed with the aim of assessing the level of reproducibility of FFPE sample preparation $(110,113)$.

Another important factor in on-tissue digestion is the chemical microenvironment of different regions within a tissue, which has been shown to cause differences in digestion dynamics (111). Furthermore, these differences in the chemical nature of the tissue extend to all chemical surface reactions, even beyond digestion. It has been demonstrated that regional differences in molecular compositions can also affect ionization efficiency due to ion suppression effects (57). Although all of these effects must be better characterized to draw better quantitative conclusions, ion suppression effects are not necessarily negative as they are specific to each tissue and therefore help to differentiate tissue types and enable tissue classification. However, these effects might become a problem for validation by other analytical platforms that try to reproduce the same results but are not "positively" affected by the on-tissue ion suppression (e.g. LC-MS).

All the factors mentioned above influence reproducibility and hence measures or quality controls are desirable that could correct for these effects, or at least indicate the quality of the tissue. The description of a sample's properties can also be extended to its quality in terms of conservation of the molecular state of the tissue at the time of sampling. A few laboratories have investigated the effects of degradation and aging on MSI data on the molecular content of the samples $(279,280)$. Significant changes have been found in tissues to occur within $30 \mathrm{~s}$ at the protein level when exposed to room temperature (281), and even on lipids after 7 months of storage at $-80^{\circ} \mathrm{C}(282)$.

The second goal of clinical research, namely the understanding of biological and pathological mechanisms, depends on the coverage of the studied molecular class, e.g. the number of detected proteins from the whole proteome. This is currently hampered by the limited analytical depth of MSI in terms of coverage and identification capabilities. The lack of analytical purification and separation steps makes direct on-tissue identification through parent ion isolation and fragmentation challenging.

In consequence, small molecules and peptides are now mainly identified by mass matching of the observed $m / z$ value, usually determined with high-mass resolution and accuracy mass spectrometers such as FT-ICR, with public or sample-specific databases created from parallel extract-based LC-MS/MS experiments $(109,118)$. However, the number of identified molecules is still one to two magnitudes lower than from state-of-the-art bottom-up proteomics and metabolomics experiments. A promising way of combining the analytical depth of liquid extraction techniques and MSI is local extraction such as LESA. It has already been successfully applied in the spatial profiling of proteins and metabolites on tissue surfaces $(69,283)$. However, there is still a trade-off between spatial resolution and analytical depth. 
Another issue in MSI is data analysis and management. With the availability of highthroughput MSI systems, high spatially resolved 2D or 3D datasets will become standard, including in clinical research. And as the data gain in quantity, the full exploitation of these datasets will require many innovations in processing software. For instance, single-cell MSI must be matched by single-cell virtual micro-dissection. The latter can only be enabled by new, accurate routines for the coregistration of the histological image in order to lower recent coregistration error (284), and by the automated annotation of the histological images by algorithms, as manual annotation will become unfeasible on a single-cell level. This is especially true for 3D-MSI datasets, which face an additional challenge at the singlecell level: improving the alignment between the different consecutive slides in order to make the alignment error smaller than the MSI pixel size.

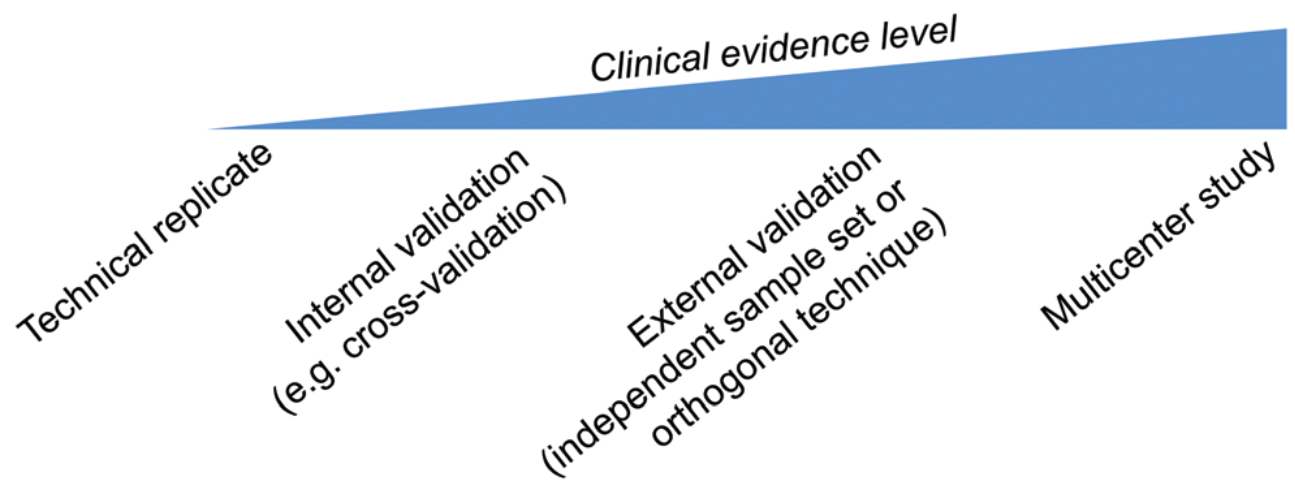

Figure 6. Measures to assess clinical evidence levels of potential biomarkers. For a successful translation, results from clinical research have to prove reproducible by a proper validation. There are different strategies, which provide different levels of evidence for the correctness of the original finding. The lowest level of evidence is provided by technical replicates. But most of validations performed in biomarker discovery studies make use of either an internal validation or external validation step. The internal validation can involve the testing of the accuracy of a uni- or multivariate marker using cross-validation techniques, or the use of another assay for trying to read out the same information from the same samples. An external validation includes an independent sample set and maybe performed by another assay. But the highest level of evidence is provided by multi-centre studies on several independent sample cohorts.

\section{Conclusions}

The cytological and histological specificity offered by MSI (fig. 1), while leaving tissue intact, has made it a unique tool in biomedical research. As shown in this review, many studies have made use of this property to address research questions which deal with complex structures in tissues such as brains, eyes, joints, wounds, and atherosclerotic plaques, etc. But the driving field of activity in MSI is and has been oncology, where MSI has allowed the extraction of tumour-specific molecular information for various purposes, such as biomarker discovery for diagnosis and prognosis, investigation of intra-tumour heterogeneity, classification of tumour margins, and the imaging of anticancer drugs in animal models (fig. 4), thereby covering all stages of translational research from pre-clinical to clinical research (fig. 5). Particularly because of its capability to visualize exogenous compounds and their metabolites in tissues, MSI has become extremely valuable for 
pharmaceutical and biotechnology companies. Therefore, the interest in the technology is growing, which also drives technical developments in the field.

MSI has undergone many technical improvements in the last few years. New atmospheric pressure ion sources have gathered interest in MSI, especially with the introduction of DESI (fig. 2). Sample preparation-free measurements make it possible now to obtain MSI data minutes after sectioning. But MALDI-MSI has also caught up. MALDI-TOF systems are now able to deliver in the same timeframe even higher spatially resolved molecular images, though at the expense of sample preparation. Both developments now allow clinical research involving large sample cohorts in a high-throughput manner (fig. 3). If MSI can overcome its final limitations, by proving sufficient reproducibility in between-centre studies (fig. 6) and improving its molecular coverage -especially the proteome- and identification, then it will not only continue to have a large impact in clinical and pre-clinical research, but will also make the final step from research to clinical application. 



\section{Chapter 3 \\ Multi-site round robin study of rapid evaporative ionization mass spectrometry}




\section{Abstract}

Rapid evaporative ionization mass spectrometry (REIMS) is a direct tissue sampling technique that has shown promise in differentiating tumour from normal tissue. To integrate this technique into routine surgical workflows, reproducibility of the apparatus, methodology and tissue classification algorithms need to be evaluated over multiple sites. Methods were harmonized using single-source reference material at 4 centres with identical equipment: Imperial College London (UK), Waters Research Centre (Hungary), Maastricht University (The Netherlands) and Queen's University (Canada). Four centres analysed 5 different types of meat sample, and 3 sites analysed samples of human normal breast and invasive breast cancer, which were validated by histopathology. Tissue recognition models were created at each centre using principal component analysis-linear discriminant analysis (PCA-LDA). Cross validation by centre ranged from 70 to $100 \%$ correct classifications for food-grade meat samples, revealing differences in meat by country. For breast tissue, correct classification rates averaged $94 \%$, but could be improved to $97 \%$ when using two centre's data for model creation. Despite inevitable variability between samples from different patients and sites, invasive breast cancer can be accurately detected by REIMS in multiple countries using models based on data from other countries. This work is anticipated to inform the design of breast cancer recognition models for future clinical use.

\section{Introduction}

Rapid evaporative ionization mass spectrometry (REIMS) is an emerging technology based on mass spectrometric analysis of aerosol generated during the thermal ablation of biological samples (285). REIMS is capable of quasi real-time, in situ classification of samples based on lipid profiling using pre-built spectral library-based classifiers $(286,287)$. The wide variety of sample types analysed by REIMS includes: food items (288-292), microorganisms (293-296), cell lines (297) and biological tissues $(29,298)$. This renders REIMS a promising tool for food fraud detection, infection diagnosis, biological research screening or histopathology diagnosis, respectively.

The quasi real-time tissue classification appears especially relevant for clinical care in the context of cancer surgery where the objective of the oncologic surgeon is to remove all the tumour tissue with a surrounding border of normal tissue, referred to as the margin. The size of the margin determines the success of the surgery, therefore the surgical decisions made in the operating room are critical for patient outcome (299). Currently, success of the surgery can only be determined postoperatively. The resection specimen has to be processed for histology, and the margins have to be examined by a pathologist. A surgery considered unsuccessful can have substantial consequences on patient outcome; the patient might need to undergo complementary treatments such chemotherapy, radiotherapy or even reoperation to treat the remaining cancer. Given the time-consuming nature of histopathology-based diagnosis of tissue, an intraoperative tool with quasi realtime readout for tissue classification at the molecular level is warranted - especially if it does not require changes to standard-of-care practice in the operating theatre $(29,298)$. To 
date, accurate differentiation of tumour versus normal tissue types by REIMS has been reported on multiple applications, including breast (300), colon (301), ovarian (302) and cervical (303) pathologies, just to name a few. These promising research results suggest that REIMS could be a valuable clinical tool to support intraoperative decision-making, and has the potential to improve outcomes for patients with cancer being treated with surgery.

The implementation of the REIMS technology for routine diagnostic use at multiple sites can face potential challenges such as differences in site-specific analytical setups (i.e. heat generators) or site-specific biological materials (i.e. tumour heterogeneity), that could impact the accuracy of tissue classification models compiled at a single site. Therefore, the analytical performance of the REIMS technology (i.e. robustness), and the performance of statistical models applied to tissue classification need to be investigated to evaluate the potential use of REIMS in routine clinical applications across several sites. Importantly, REIMS technology needs to be evaluated in the context of clinical and pathology workflows, to enable validation of REIMS spectra acquired from clinical samples, and to study the feasibility of future intraoperative use.

The objectives were to evaluate intra- and inter-site repeatability, reproducibility and robustness applied to the classification of biological tissues; including non-clinical and clinical samples. First, the REIMS spectra generated from selected reference samples acquired on 6 instruments in 4 sites (UK, Hungary, Netherlands (NL), Canada) were compared to evaluate the robustness of the REIMS technology (fig. 1a). Then, the ability of the methodology to classify four site-specific meat samples by building site-specific classifiers was assessed. Finally, the same approach was applied to clinical samples collected and analysed across the different sites from patients undergoing surgery for invasive breast cancer (fig. 1b). This work supports the vision that REIMS technology can be implemented into surgical environments around the world for routine, intraoperative margin assessment during cancer surgery. 
a)

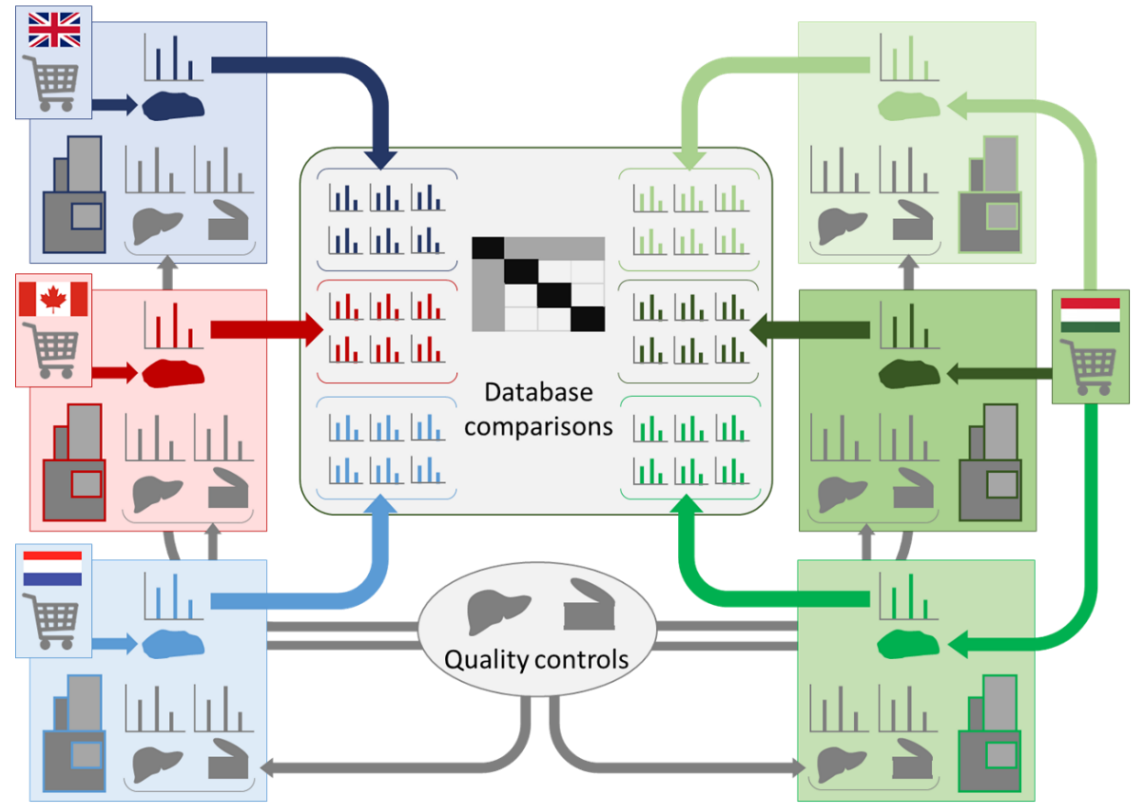

b)

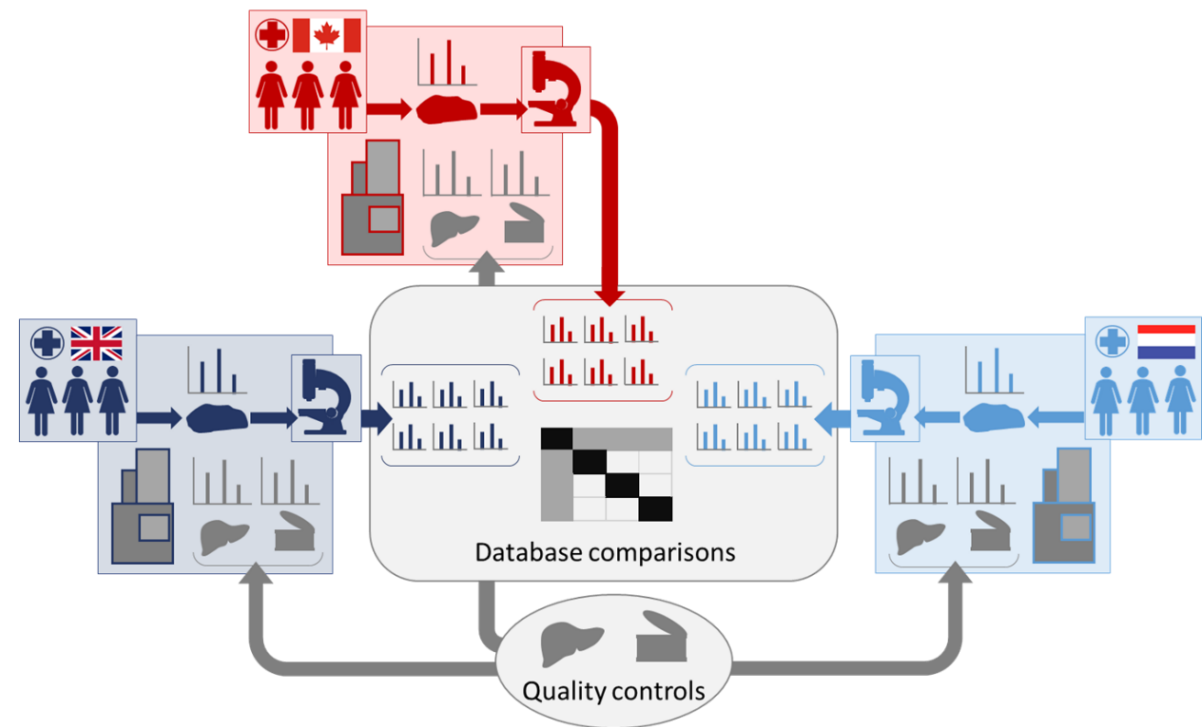

Figure 1. Workflow for multicentre study. Quality control material including 2 batches of single-source pork liver and reference meat homogenate was used to harmonize REIMS methodology at each centre. (a) Local sources of food-grade meat samples were used to create models at each site that were tested using the other centre's data. (b) A similar approach was used to compare REIMS data among clinical sites using pathology-validated human breast tissue. 


\section{Material and methods}

\section{Materials and reagents}

Isopropanol and water (UPLC/MS grade) were purchased from Honeywell (VWR, NL). Leucine-encephalin (LeuEnk) was purchased from Sigma-Aldrich (St. Louis, US). Sodium hydroxide was purchased from Merck (Darmstadt, Germany).

\section{Samples and logistics}

Non clinical samples. Samples consisted of:

1) NIST reference meat homogenate (Standard reference material ${ }^{\circledR}$ 1546a, National Institute of Standards and Technology, NIST);

2) Two individual batches of pork liver procured by Imperial College London (Centre 1 (C1), London, United Kingdom and shipped to 3 other participating centres comprising Waters Research Centre, Budapest, Hungary (C2); Maastricht MultiModal Molecular Imaging Institute, Maastricht The Netherlands (C3); and Queen's University, Kingston Canada (C4);

3) Four types of food-grade meat samples (calf liver, chicken liver, chicken breast, turkey breast) procured from local supermarkets analysed at each site (fig. 1a). Pork liver samples were shipped from $\mathrm{C} 1$ to the other sites on dry ice and stored in low temperature $\left(-80^{\circ} \mathrm{C}\right)$ freezers until analysis.

Clinical samples. Human breast tissue was collected at 3 consortium sites affiliated with clinical centres, from patients who underwent surgery for treatment of invasive breast cancer (fig. 1b). This study was approved by local medical ethics committees: Imperial College London (C1); East of England - Cambridge East Research Ethics Committee, REC reference 14/EE/0024; Maastricht University (C3), medical ethics committee of Maastricht University Medical Centre (MUMC+) permit No. METC 16-4-168; and Queen's University (C4) Health Sciences Research Ethics Board, permit No. 6023032. After macroscopic examination, a pathologist selected tumour and/or normal tissue samples from 6-8 breast cancer surgery cases from each site. Samples were stored in a low temperature freezer ($80^{\circ} \mathrm{C}$ ) until REIMS analysis, described below.

\section{Sampling with diathermic knife}

All tissues were allowed to reach room temperature before analysis and placed on a return electrode (i.e. conductive silicon). If necessary, samples were humidified by wetting with deionised water. Electrosurgical dissection was carried out using commercial electrosurgical generators (in Maastricht/Queens: Covidien Ltd. Force FX, Ireland; in ICL: Covidien Ltd. Triad, Ireland; in WRC: ERBE Elektromedizin GmbH ICC-350, Germany) providing powercontrolled sinusoidal $330 \mathrm{kHz}$ alternating current. Smoke was generated by mean of a custom hand-piece provided by Waters Research Centre (Budapest, Hungary) with a smoke evacuating line (tubing system). The signal coming from the smoke lasted for a few seconds (around 3-5 seconds). 
Settings for the sampling of non-clinical samples. The generator was used in "cut" mode with a power setting optimized to the electrosurgical generators and the samples (i.e. $10 \mathrm{~W}$ for the NIST reference meat homogenate, $20 \mathrm{~W}$ for the pork liver and food-grade meat samples). In order to maximize reproducibility, the burns made with the diathermic knife using a blade were carried out while keeping the knife in a semi-vertical position on top of the tissue. NIST homogenate samples and liver distributed from C1 were measured before and after each series of breast measurements (see next paragraph) and used as quality control to assess instrumental variability across sites. Measurements were performed two times per day, on two consecutive days for each sample. Each measurement consisted on a few burns ( 5 burns in general), each burn lasting for a couple of seconds ( 4 to 8 seconds in general).

Settings for the sampling of clinical samples. The generator was used in "cut" or "coagulate" ("coag") mode with a power setting optimized to the electrosurgical generators (15 to 70 $\mathrm{W}$ in "cut" mode 10 to $30 \mathrm{~W}$ in "coagulate" mode). The burns were made with a diathermic knife using a half cut blade to optimize smoke aspiration into the hand piece.

\section{REIMS - qTOF instrumentation}

Data acquisition were performed on benchtop mass spectrometers (Xevo G2-XS QTOF, Waters, Manchester, UK) mounted with a REIMS source (version III) installed at each site. Operating parameters were kept constant between the four different sites. All parameters were set and instrument status was checked according to the checklist distributed to all sites and shown in figure 2. Instruments were connected to a 7 bar gas (pressurized air or Nitrogen) supply. TOF and backing pressures were in the range of $<e^{-7}$ and 1.3 mbar, respectively. The smoke produced by electrocautery was aspirated via a Venturi pump connected to the REIMS interface. The heated coil in the REIMS source was kept at $8-900^{\circ} \mathrm{C}$. Data were acquired in "sensitivity" and negative ionization modes within the mass-tocharge $\mathrm{m} / \mathrm{z}$ range of $100-1500$. Mass resolution was above 15.000 full width at halfmaximum (FWHM). The calibration of the instrument was performed or checked before each measurement series with a solution of sodium formate. A solution of LeuEnk at a concentration of $0.05 \mathrm{ng} / \mu \mathrm{l}$ (prepared in isopropanol) was continuously infused during acquisition at a flow rate of $150 \mu \mathrm{l} / \mathrm{min}$ for external lock-mass correction. MS/MS fragmentation of molecular species was carried out using nitrogen as collision gas with 30$45 \mathrm{eV}$ collision energy according to the exact mass and molecular species type.

\section{Histopathology examination of the clinical samples}

After REIMS analysis, remaining tissues were fixed in formalin (Unifix, Klinipath) and embedded in paraffin. Tissue sections were then stained by haematoxylin and eosin (H\&E) and examined by a breast pathologist. A pathologist at each centre examined the tissues obtained and analysed at their respective centre. Tissues were assessed for the proportion of either tumour or normal adipose surrounding the area analysed by REIMS. Spectra 
acquired from tissue containing at least $30 \%$ invasive cancer were labelled as invasive breast cancer, and spectra containing only $100 \%$ normal were labelled as normal breast tissue. Spectra associated with 5-25\% invasive breast cancer were excluded.

\begin{tabular}{|c|c|c|}
\hline \\
\hline \multicolumn{3}{|c|}{$\begin{array}{l}\text { - Make sure the instrument has been in operate mode for } \\
30 \text { minutes } \\
\text { - If there is no signal on the instrument when solvent is } \\
\text { flowing, try the following in the given order, and check if } \\
\text { there is signal after each step: }\end{array}$} \\
\hline \multicolumn{3}{|c|}{$\begin{array}{l}\text { - Change to other polarity mode }(+) \text { and back } \\
\text { - Put MassLynx into instrument standby (red), then } \\
\text { switch to operate again } \\
\text { - Restart electronics }\end{array}$} \\
\hline \multicolumn{3}{|l|}{ Instrument settings } \\
\hline \multicolumn{3}{|c|}{ - The correct .ipr file is loaded } \\
\hline \multicolumn{3}{|l|}{ - Negative mode } \\
\hline \multicolumn{3}{|l|}{ - Sensitivity mode } \\
\hline \multicolumn{3}{|l|}{ - Collision gas is ON } \\
\hline \multicolumn{3}{|c|}{ - Collision Energy is OFF (Instrument tab) } \\
\hline \multicolumn{3}{|c|}{ - Correct scan time is set } \\
\hline \multicolumn{3}{|c|}{ - Backing pressure is $>1.5$} \\
\hline \multicolumn{3}{|c|}{ - Collision pressure is between $1.5^{*} 10^{-1}-2.5^{*} 10^{-1}$} \\
\hline \multicolumn{3}{|c|}{$\begin{array}{l}\text { - TOF pressure is below } 1.1 * 10^{-6} \text {, but ideally below } 9 * 10^{-7} \\
\text { - Check the following instrument parameters are set, } \\
\text { when MS Profile Type is set to Auto P: }\end{array}$} \\
\hline \multirow{2}{*}{$\begin{array}{l}\text { Sampling Cone } \\
\text { Source Offset }\end{array}$} & 40 & RFIMS tah \\
\hline & 30 & \\
\hline Collision RF Offset & & \\
\hline & & \\
\hline & 150 & \\
\hline MS Profile Type & Auto P & \\
\hline
\end{tabular}

\section{REIMS source (commercial)}

- Heater is turned ON

- Small IPA capillary is in place

- The impactor holder assembly is open

\section{General}

- After starting LeuEnk in IPA, LeuEnk peak is visible around 554.2

- There is no peak splitting of 554.2

- Check the resolution is $>15000$

- Correct mass range is set (usually 50-1200)

- Check if the file duration is set to 0 when starting a file

\section{Regular check}

- The instrument has been calibrated/calibration was checked that day

- The instrument has been calibrated since last electronics shutdown/reboot

- The detector was calibrated in the past month

\section{Breast ex vivo trial check}

- The overall TIC (during burns) $>5 \mathrm{e}^{7}$

- LeuEnk intensity level per scan $1 e^{5}-5 e^{6}$ at the beginning

- LeuEnk intensity level per scan $1 e^{5}-5 e^{6}$ at the end

- Tissue intensity level per scan (highest peak in the $600-1000$ range) $>5 e^{4}$

- Resolution $>15000$ at the beginning

- Resolution > 15000 at the end

Figure 2. REIMS system set-up and suitability checklist.

\section{Data analysis}

Mass spectral processing and multivariate data analysis were performed using the Abstract Model Builder (AMX) software ([beta] version 1.0.1581.0, Waters Research Centre, Budapest, Hungary). All mass spectra were processed as follow: i) background subtracted; ii) mass shift corrected against the reference peak of deprotonated LeuEnk $[\mathrm{M}-\mathrm{H}]^{-}$at $\mathrm{m} / \mathrm{z}$ 554.2615 ; iii) binned to $0.1 \mathrm{Da}$ (within the mass range $\mathrm{m} / \mathrm{z} 600-1000$ - corresponding to the region of abundant phospholipids and triglycerides); iv) normalized against the total ion count (TIC). Multivariate analysis was based on principal component analysis/linear discriminant analysis (PCA/LDA). PCA was performed with a maximum of $n=25$ dimensions and LDA with $\mathrm{n}-1$ dimensions where $\mathrm{n}$ corresponds to the number of variables introduced 
in the model. Cross-validation tests were performed by building the site specific classifiers to recognize the data generated on the other sites. Data points were marked as outlier if they deviate $5 \times$ standard deviation (SD).

\section{Results}

\section{Analyst-dependent REIMS metabolic patterns}

First, it was determined if there were any differences in lipid patterns acquired by REIMS that were analyst-dependent. Seven analysts (from across each of the 4 centres) conducted multiple REIMS samplings on the same instrument at C3 and on batch 1 pork liver in an unbiased manner. None of the analysts were permitted to observe the specific sampling technique used by the other analysts, in order to avoid the introduction of sampling bias. PCA of at least 3 burns per analyst revealed no separation of patterns based on the individual analyst. This observation confirmed that slight differences in how REIMS burns were conducted by each centre's analyst was unlikely to be a source of variability in between-centre comparison of patterns in subsequent experiments described in figure 3 .

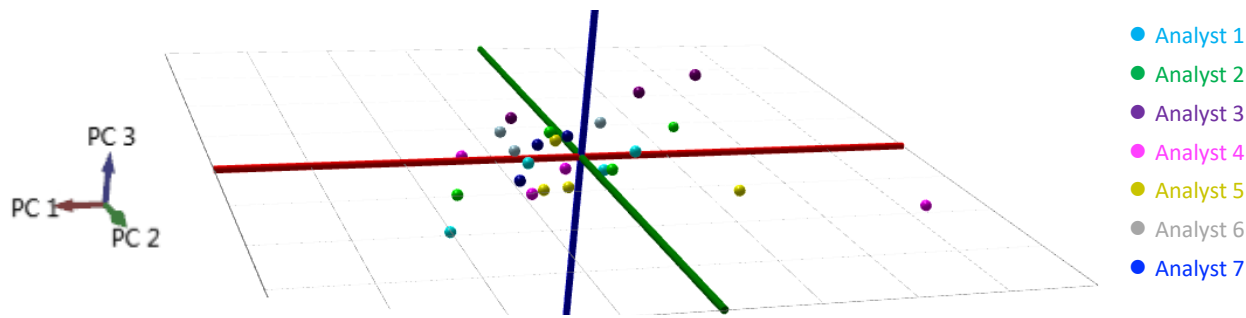

Figure 3. Analyst-dependent differences in REIMS spectra. Seven analysts from all four centres conducted at least three REIMS analyses on batch 1 pork liver at C3. The PCA score plot of the $m / z 600-900$ region of the mass spectra revealed insignificant analyst-dependent differences associated with sampling technique.

\section{Multi-site characterization of pork liver and NIST meat homogenate}

With analyst-dependent differences ruled out as a major source of potential variability among centres, "Batch 1 pork liver" was then analysed at each centre (C1,C2,C3 and C4) using the same instrumental setup. For this purpose, the mass spectra acquired across the different centres were compared qualitatively. Mass spectra comprised a range of fatty acids (FAs) and phospholipid species including phosphatidylethanolamines (PEs) and phosphatidyl inositol (PI), as shown in figure 4.

These species were identified using accurate mass measurements and MS/MS fragmentation of the molecular ions in pork liver (fig. 4). Tandem MS fragmentation spectra were compared to reference literature (304). Most of the FA species observed included: $m / z$ 255.23, [FA(16:0)-H] $m / z$ 279.23 [FA(18:2)-H] ; $m / z$ 281.25 [FA(18:1)-H] $; ~ m / z ~ 283.26$ [FA(18:0)-H]. Major glycerophospholipid species observed included: $m / z 699.50$ [PA(36:2)$\mathrm{H}^{-}$and $\left[\mathrm{PE}(34: 1)-\mathrm{NH}_{4}\right]^{-}, 725.51$ [PE(36:2)- $\left.\mathrm{NH}_{4}\right]^{-}, 742.53[\mathrm{PE}(36: 2)-\mathrm{H}]^{-}, 766.54$ [PE(38:4)-H]', 
$865.58[\mathrm{PI}(36: 0)-\mathrm{H}]^{-}$, and $\mathrm{m} / \mathrm{z} 885.55$ [PI(38:4)-H] comprising phosphatidic acids, phosphphatidylinositols and phosphatidylethanolamines. Overall, there were striking differences in the ratios between fatty acids and phospholipids between sites, with C2 exhibiting the greatest fatty acid-to-phospholipid ratio and $\mathrm{C} 4$ exhibiting the lowest (C2 > C3 > C1 > C4). Furthermore, distinct differences in the ratios between phospholipids were apparent within the phospholipid range, which was used for model creation and classification. For example, $\mathrm{m} / \mathrm{z} 766.54$ was the most abundant species in spectra acquired at $\mathrm{C} 2$ and $\mathrm{C} 4$, but $\mathrm{m} / \mathrm{z} 699.50$ was the most abundant in C1 and C3 spectra, based on the same piece of pork liver (fig. 4). The 3D-PCA plot displayed in figure 5 a revealed centredependent grouping of spectra based on the phospholipid range. However, while slight variability in spectra from the same sample between centres might be inevitable, the more important consideration is how well two different sample types could be differentiated from each other between the 4 centres. Therefore, the spectra acquired from NIST meat homogenate were plotted alongside those from the batch 1 pork liver (fig. 5b). This revealed distinct groups of spectra associated with either pork liver or NIST meat homogenate (alongside the PC2 axis) regardless of centre, although centre-dependent sub-groups of pork liver spectra were still clearly distinguishable.

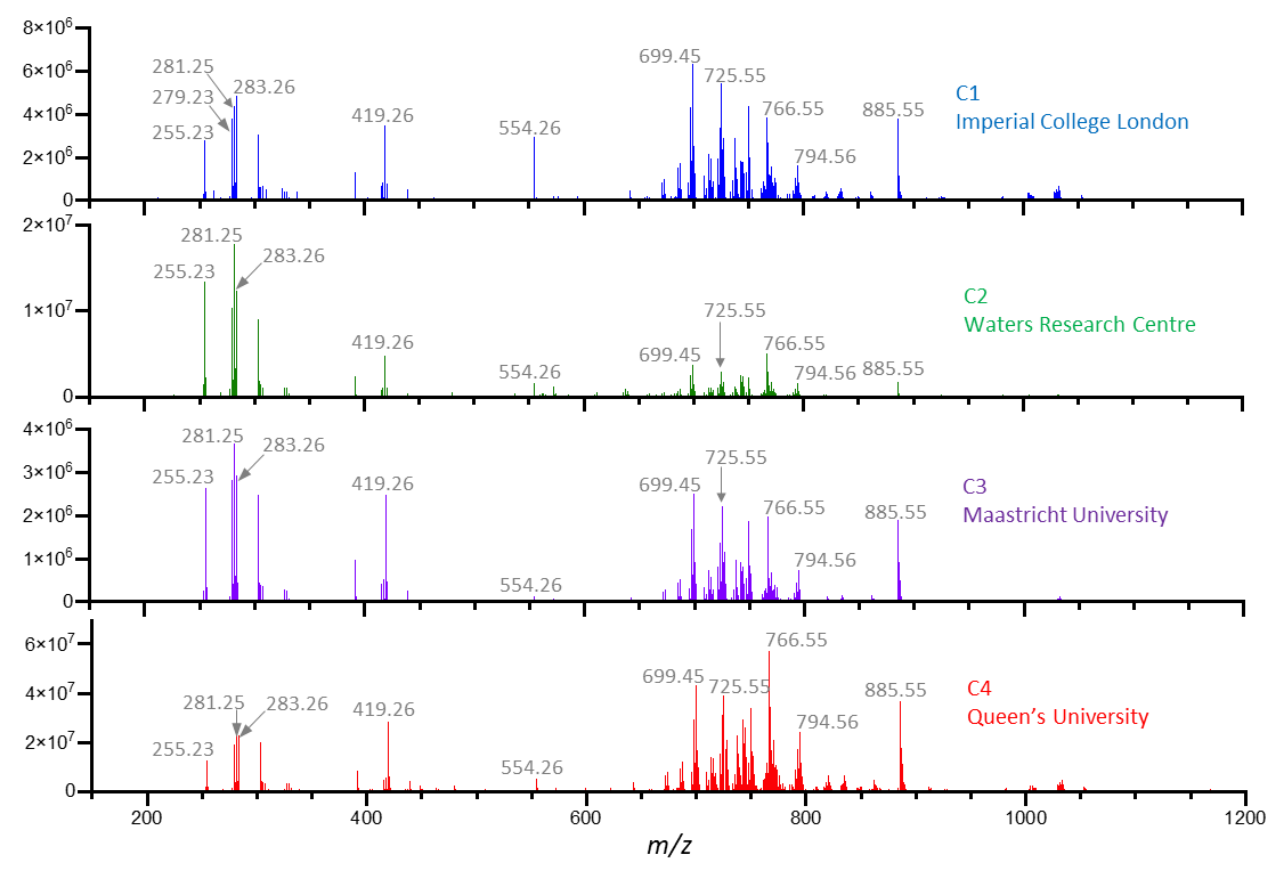

Figure 4. REIMS spectra of single-source pork liver determined at each centre. A single source of pork liver was distributed by $\mathrm{C} 1$ to all other centres. REIMS spectra from each centre are compared over a broad mass range. Each representative spectrum shown is the average of 6-7 individual scans. 
a)

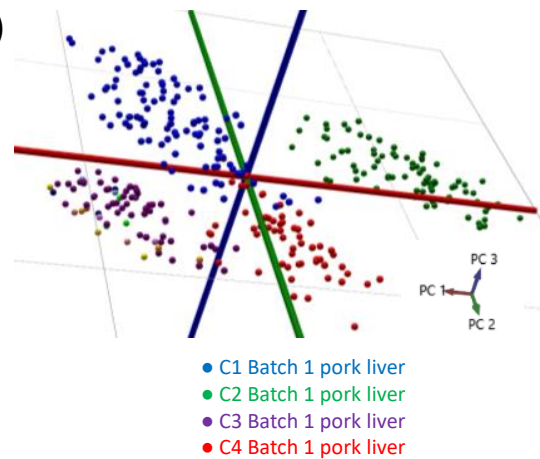

b)

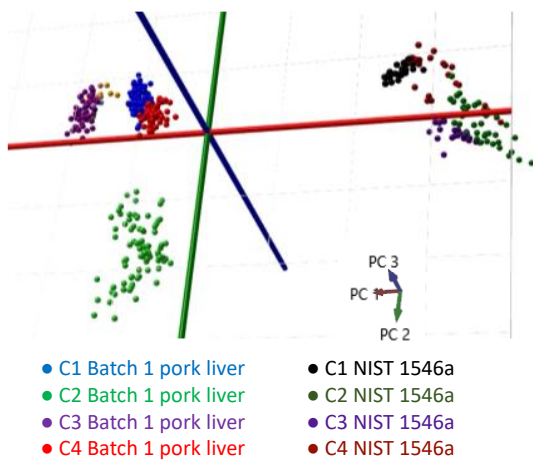

Figure 5. Multivariate analysis of REIMS spectra from single-source pork liver and NIST meat homogenate determined at each centre. (a) Principal component analysis (PCA) was used to study variation in multiple REIMS spectra acquired from a single source of pork liver (batch 1) distributed to all centres. Data points acquired at C3 also includes analyses conducted on the same pork liver by 7 analysts representing all consortium sites (see fig. 3). (b) A combination of principal component analysis was used to study class-dependent differences in spectra from batch 1 pork liver and NIST meat homogenate among centres.

To minimize the centre-dependent variability in spectra acquired from the same reference material, all instrument data acquisition parameters were unified and a pre-acquisition checklist was created to ensure that all instrument settings were harmonized prior to each analysis (fig. 2). The possibility that differences in in-source fragmentation among centres might explain variability in spectral properties was investigated. Therefore, the fragmentation pattern of the lock-mass compound leucine-encephalin ( $\mathrm{m} / \mathrm{z} 554.2615)$ was examined and compared this to spectra from all centres as a surrogate indicator of potential phospholipid fragmentation. Major fragments of leucine-encephalin include $\mathrm{m} / \mathrm{z} 236.1035$ and 293.1250, but these ions were not observed in the pork liver spectra from any centre (fig. 6). While it could not rule out phospholipid fragmentation occurring at the sampling site (as opposed to in-source), all centres used identical diathermy pencils and return electrodes for all sampling events. Differences in pre-analytical handling of the pork liver (e.g. storage, thawing) could also have contributed to centre-specific differences in spectra.

With instrumentation and methodology harmonized among the four centres, the pork liver and NIST meat homogenate comparison was repeated. Centres 1-3 used a second batch of pork liver, however this sample was not available at C4, where batch 1 was used instead. From the PCA analysis of the pork liver spectra in figure 7a, it was evident that betweencentre variability was reduced significantly towards the within-centre variability, in comparison to the previous analysis shown in figure $\mathbf{5}$. It can be concluded that the remaining between-centre variability is insignificant, because spectra from the meat homogenate and pork liver (regardless of batch) grouped into distinct clusters on PCA analysis, and centre-specific subgroups could not be easily discerned (fig. $\mathbf{7 b}$ ). 


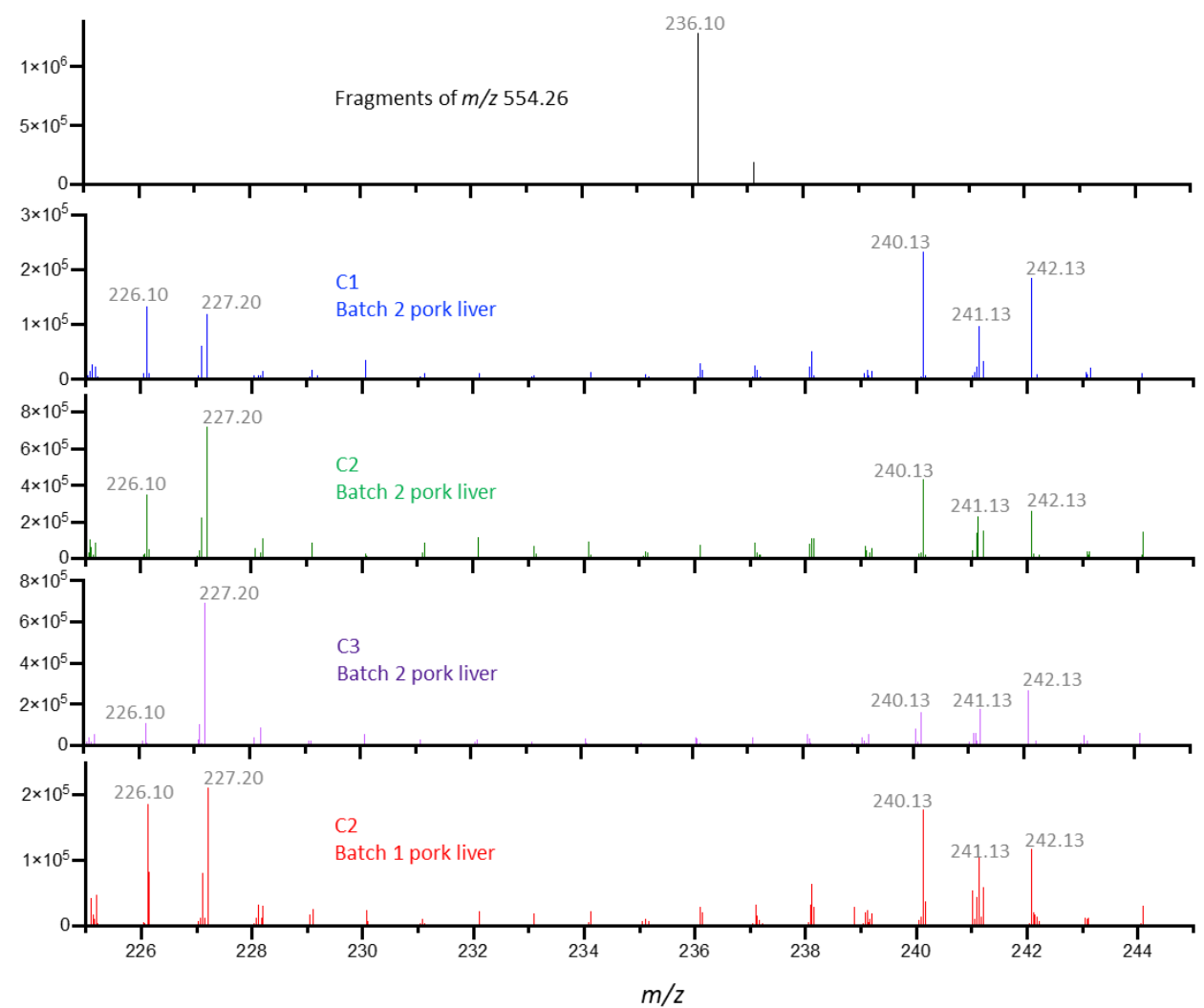

Figure 6. Fragmentation of leucine-encephalin. Leucine-encephalin was used as an external lockmass compound in all analyses. We compared abundance of the most significant leucine-encephalin fragment $\mathrm{m} / \mathrm{z} 236.10$ produced at collision energy of $30 \mathrm{eV}$ with spectra from batch 1 or 2 pork liver spectra to assess the likelihood that lipid fragmentation could occur at the heated collision surface of the REIMS interface.

\section{Multi-site classification of local food-grade meats}

Each centre acquired four different types of food-grade meat from local sources, which were subjected to analysis by REIMS using harmonized methodology (fig. 1a). Sufficient sample was sourced at C2 to enable analysis over three different instruments (C2-1, C2-2, C2-3), whereas all other centres continued to use a single instrument. A total of 2435 scans were selected from 487 burns, comprising:

- calf liver - 126 burns (630 scans);

- chicken breast - 105 burns (525 scans),

- chicken liver - 147 burns (735 scans),

- $\quad$ and turkey breast 109 burns (545 scans). 
The phospholipid range of spectra from locally sourced calf liver across all instruments (fig. 8) revealed variability associated with different sources of calf liver, but no significant variability associated with analysing the same calf liver on multiple instruments at C2.

a)

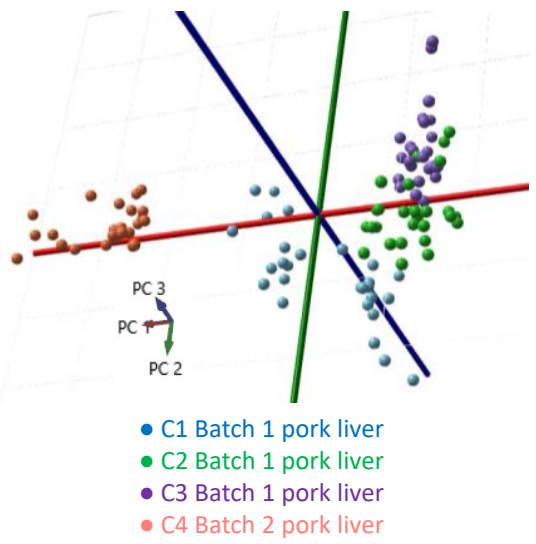

b)

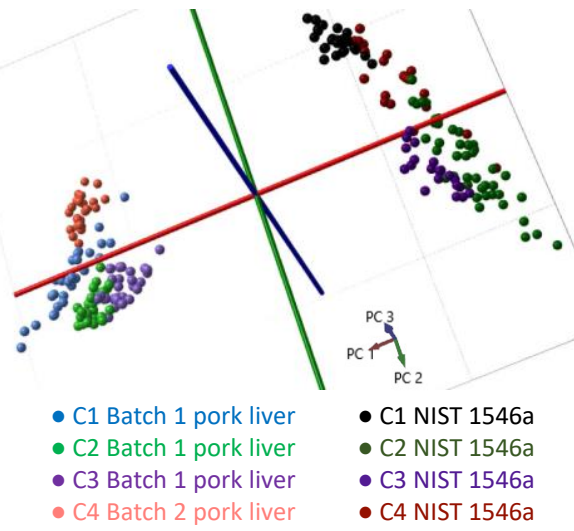

Figure 7. Multivariate analysis of REIMS spectra from pork liver and NIST meat homogenate determined at each centre using harmonized analytical conditions. After all analytical conditions were harmonized, variation in spectra from batch 2 pork liver and NIST meat homogenate were studied using either PCA and/or PCA/LDA analysis. C4 used batch 1 pork liver for this comparison.

The multivariate plots in figure 9 depict the distribution of all of the spectra using both PCA (fig. 9a) and a combination of PCA and LDA (fig. 9b) which was used for model creation, classification and testing.

Individual models using each canter's data were created and the number of correct classifications was determined when tested against data from the remaining canters (table 1). Correct classification rates ranged between $69 \%$ and $100 \%$, where $C 2$ exhibited the greatest correct classification rate and $\mathrm{C} 4$ the least, with models from $\mathrm{C} 1$ and $\mathrm{C} 3$ achieving correct classification rates of 75 and $88 \%$. Worth mentioning that the model for $\mathrm{C} 2$ was based on a larger number of spectra from three instruments, and that C4 was the only nonEuropean centre. Given that cross validation between C2's three instruments using the same source of meat was also $100 \%$, it could be concluded that incorrect classifications likely occurred because of differences in meat sources rather than instrument-toinstrument variability, especially when creating a model with North American meat and testing it against European meat. 


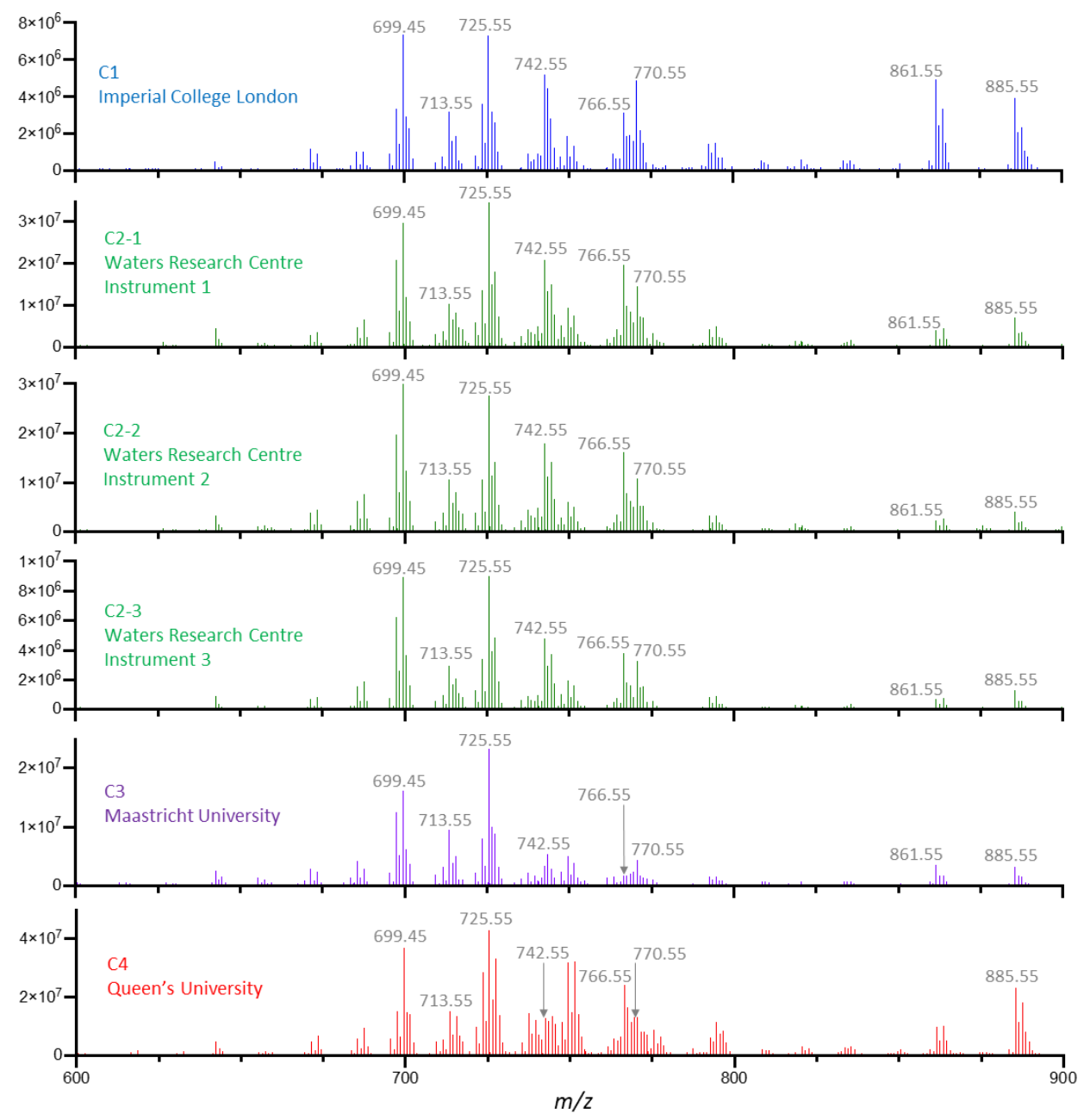

Figure 8. REIMS spectra of locally-sourced calf liver determined at each centre. Using harmonized analytical conditions, each site analysed locally-sourced food grade meats. Spectra from calf liver are presented, focussing on the phospholipid mass range, $m / z$ 600-900. For this study, $C 2$ analysed each sample on three different instruments. Representative spectra shown, are the average of 6-7 individual scans.

All incorrect classifications were based on calf liver being inappropriately classified as chicken liver, and chicken breast being incorrectly classified as turkey breast. When the pork liver reference samples were included in the analysis, each centre's model always correctly classified the pork liver, confirming that between-instrument variability was insignificant, relative to the variability associated with centre-specific sources of meat. While it was concluded that incorrect classification of meats between centres arises from differences in meat sources, it is important to examine why certain models perform better than others; given that the goal is to create a model for invasive breast cancer. In this context, it is 
noteworthy that the tissue classification model should be able to accurately recognize cases at multiple sites despite environmental and genetic factors that contribute to tissue heterogeneity.

a)

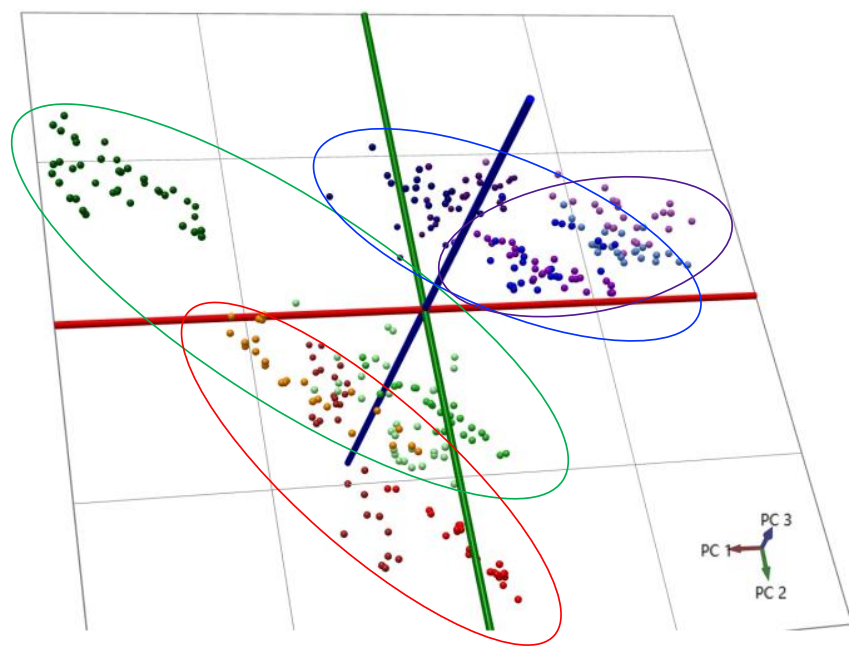

b)

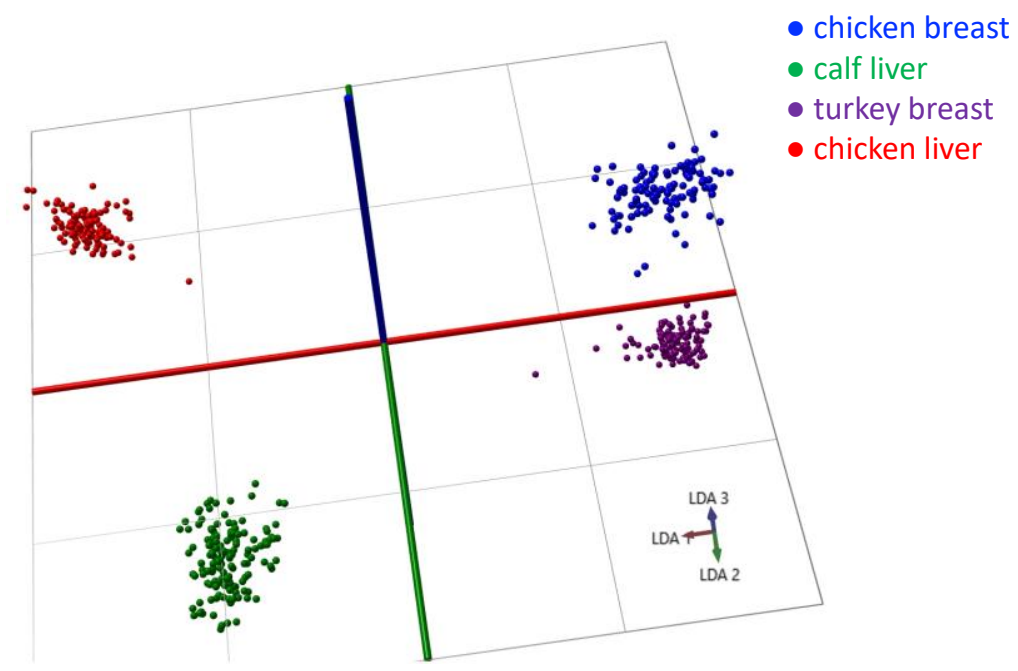

Figure 9. Multivariate analysis of REIMS spectra acquired from local sources of food-grade meat. Each centre analysed meat from local sources including, chicken breast, turkey breast, calf liver, and chicken liver. The PCA (a) and PCA/LDA (b) score plots compare the overall, and tissue-type variability among sites.

C2's meat recognition model appeared to encompass the variability associated with heterogeneity within meat types among all centres, as the model exhibited a $100 \%$ correct classification rate. As the C2 model was generated using spectra acquired over 3 instruments, it is tempting to suggest that a greater number of spectra over three 
instruments was statistically advantageous. However, a model created from a single instrument at $\mathrm{C} 2$ also exhibited a near-perfect classification rate. Even though variance of C1 was greater than C2 as determined by the standard deviation of the PCs 1-3 (fig. 10) and major phospholipid peaks (fig. 11), C1, exhibited a correct classification rate of only $75 \%$, suggesting that variance was not the only factor that contributed to the success of $C 2$ 's model. Furthermore, while it can be rationalized that differences in North American meat (C4) versus European meat would place C4's model at a clear disadvantage, this model also exhibited the lowest variance and accordingly the poorest correct classification rate. On the other hand, C4's spectra were well classified by most other models. The variance of C3's model was slightly greater than $\mathrm{C} 4$ and exhibited a significantly improved correct classification rate of $88 \%$ as compared with $69 \%$. Taken together, the overall success of C2's model was likely due to a combination of variance and that it appeared to be the best representative or 'average' source of meat compared to other sites.

Table 1. Correct classification rate for all types of food-grade meat using models created at individual sites.

\begin{tabular}{|l|c|c|c|c|c|c|c|}
\hline \multirow{2}{*}{$\begin{array}{l}\text { Model used for } \\
\text { training } \downarrow\end{array}$} & \multicolumn{7}{|c|}{ Correct classification rate (\%) } \\
\hline C1 & Total & C1 & C2-1 & C2-2 & C2-3 & C3 & C4 \\
\hline C2 (all) & 75.6 & & 64.7 & 50.0 & 50.0 & 100.0 & 75.0 \\
\hline C2-1 & 100.0 & 100.0 & & & & 100.0 & 100.0 \\
\hline C3 & 97.1 & 95.6 & & 100.0 & 100.0 & 100.0 & 94.6 \\
\hline C4 & 88.3 & 99.1 & 73.3 & 50.0 & 92.9 & & 100.0 \\
\hline
\end{tabular}

${ }^{1}$ denotes model created from all three instruments at C2.

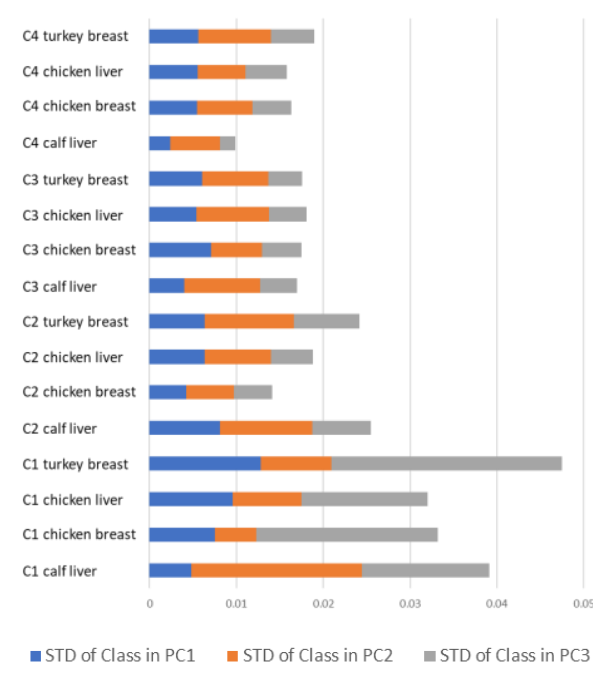

Figure 10. Standard deviation (STD) of principal components (PC).

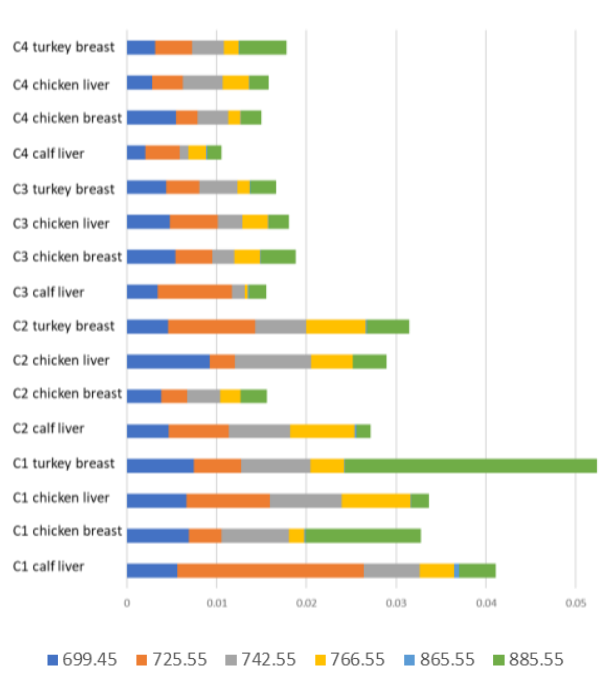

Figure 11. Standard deviation of phospholipid peaks (multiple peaks). 


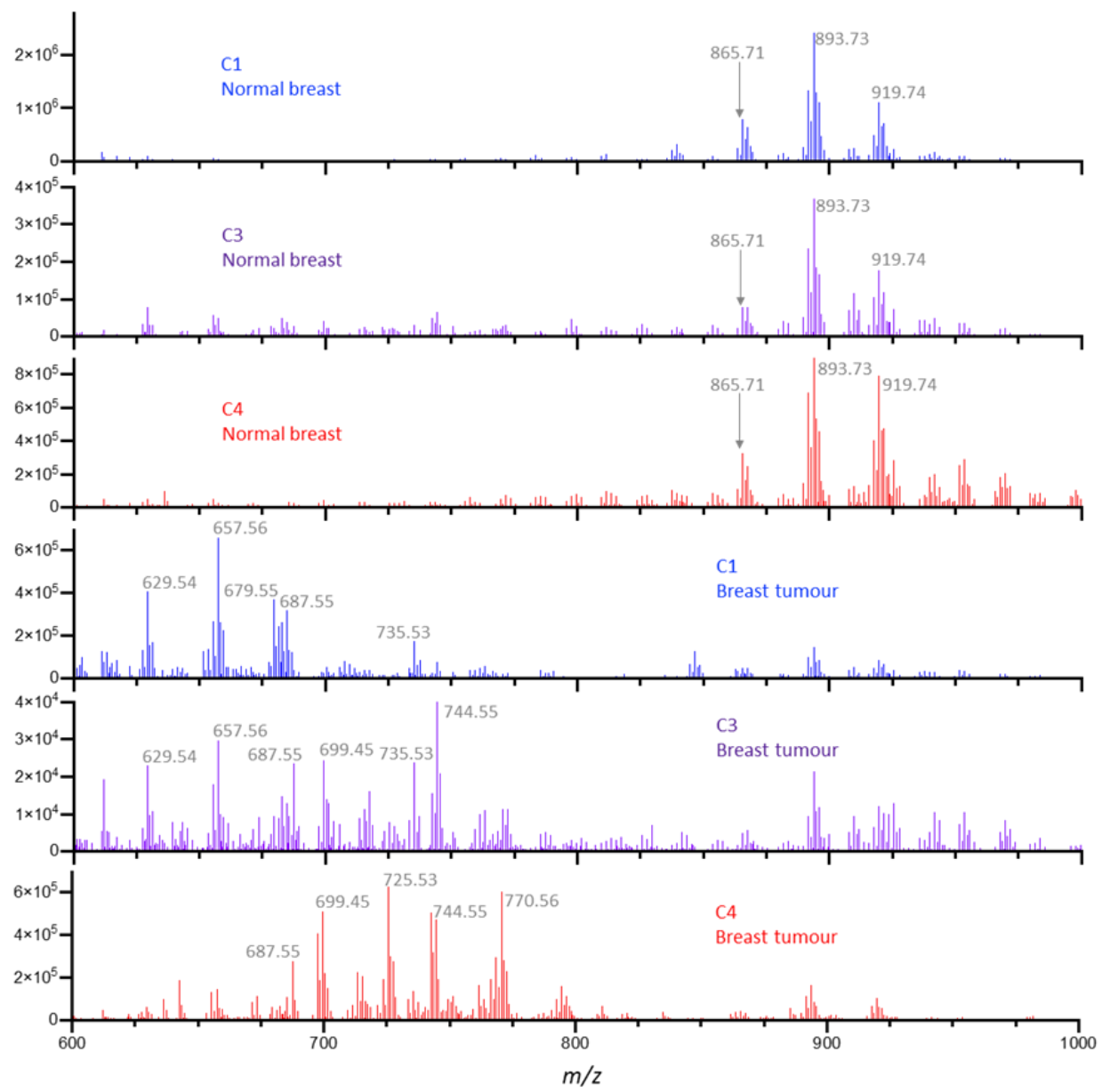

Figure 12. REIMS spectra from human breast tissue samples. Representative REIMS spectra from either normal or invasive breast cancer acquired from patients being treated at the 3 consortium sites affiliated with clinical centres. The spectra shown are 1s scans acquired using cut mode, subjected to background subtraction and lockmass correction. The tissue type was determined by a breast pathologist at each site.

Comparison of REIMS spectra of human breast tissue and invasive breast cancer across sites. Towards the goal of translating REIMS for routine use in surgical settings around the world, human breast samples collected from patients undergoing breast cancer surgery at the three sites affiliated with clinical centres (C1, C3, C4) were analysed (fig. 1b). Each site obtained invasive breast cancer and/or normal breast adipose samples from specimens resected from 6-8 patients. In an attempt to model different surgical workflows, all samples were analysed using both cut and coagulation mode where possible, acquiring a total of 260 spectra. Not surprisingly, the intensity of the REIMS signals differed greatly among the sites, and the signal-to-noise ratio of human breast measurements was significantly lower 
compared to food-grade meats due to several factors, including: the heterogeneity of breast tissue, the smaller-sized samples collected from surgery, and due to the challenging task of cauterizing normal adipose with a monopolar device. Representative spectra from either normal adipose or invasive cancer acquired in cut and coag are shown in figures 12 and 13.

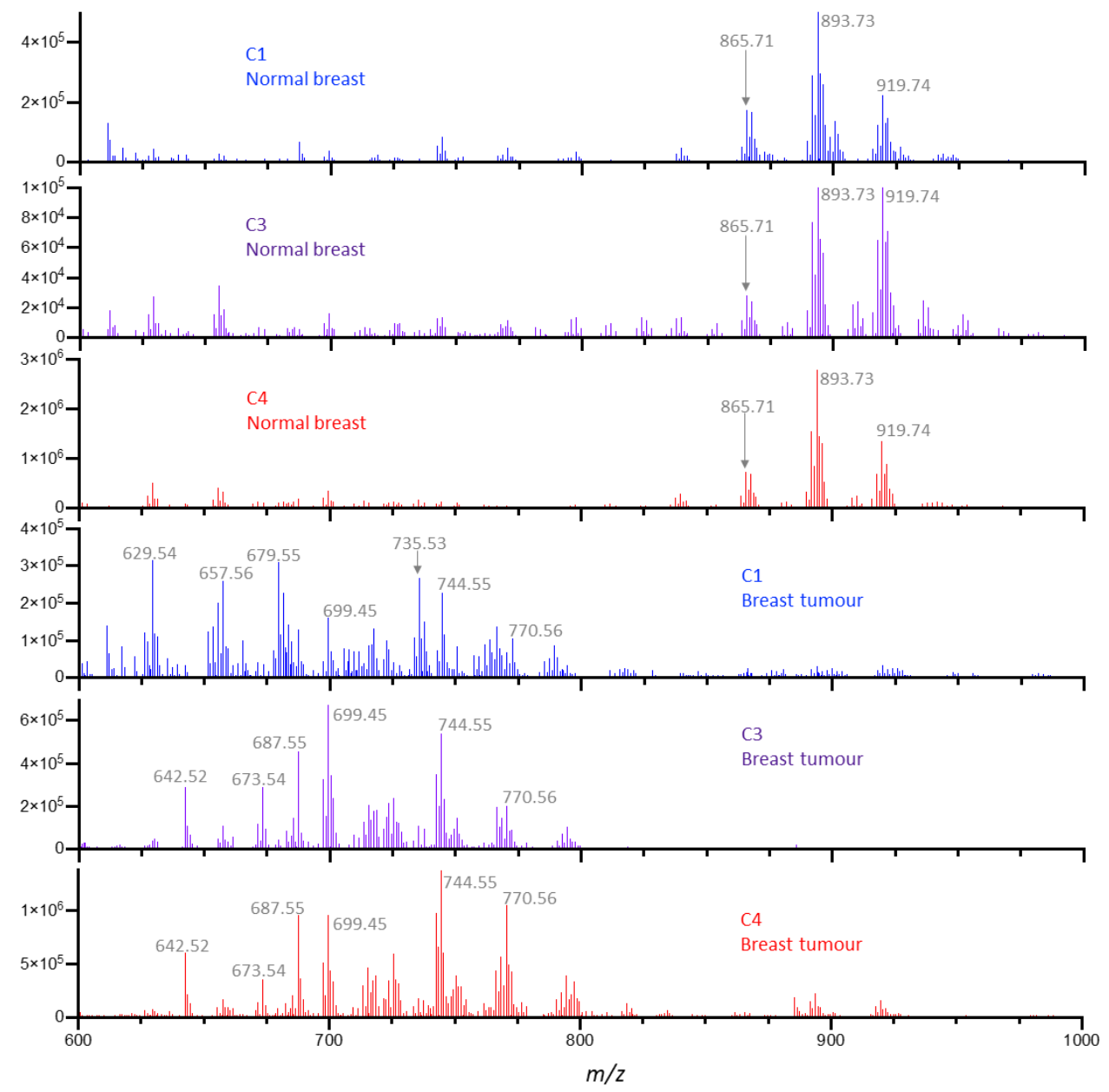

Figure 13. REIMS spectra from human breast tissue samples. Representative REIMS spectra from either normal adipose or invasive breast cancer samples acquired from patients being treated at the 3 consortium sites affiliated with clinical centres. The spectra shown are 1s scans acquired using coagulation mode, subjected to background subtraction and lock-mass correction. The tissue type was determined by a breast pathologist at each site.

Despite these challenges, we observed a remarkably consistent general pattern of $\mathrm{m} / \mathrm{z}$ ratios in adipose as compared with invasive breast cancer among all sites. For example, triglycerides ( $m / z$ 850-1000) dominated the spectra acquired from normal fatty tissue, but were mostly absent from the tumour spectra, which exhibited relatively high 
concentrations of phospholipids, in agreement with previous investigation (305). Highly abundant triglycerides observed in normal breast adipose include $\mathrm{m} / \mathrm{z} 865.71$ (TG(50:2), 893.73 (TG(52:2)) and 919.74 (TG(54:3)). Inter-sample heterogeneity in the breast cancer samples can be inferred from the variable relative abundance of specific phospholipid constituents in figures $\mathbf{1 2}$ and $\mathbf{1 3}$. These differences presumably arose from a variable combination of cells associated with tumour, normal glandular, fibrous, scar and necrotic tissue, which emphasizes the importance of histopathology annotation. To optimize the model, invasive breast cancer spectra containing at least $30 \%$ tumour cells around the sampling site were included, as well as normal spectra that were free of tumour cells based on histopathology analysis. Also, spectra were excluded if there was a discrepancy between the gross tissue label and the histopathology-assigned label, or if the intensity of the scan was lower than $2 \times 10^{4}$.

Multivariate analysis of human breast data and multi-site tissue classification. A total of $\mathrm{n}=$ 210 pathology-annotated spectra were included in the multivariate model; including:

- $\quad n=71$ invasive breast cancer (39 cut/32 coag);

- $\quad$ and $n=139$ normal adipose (82 cut/57 coag).

Figure 14 shows PCA and PCA/LDA score plots where the separation between cancer and normal is almost complete, independent of the site. A correct classification rate of $96.4 \%$ (range 92-100\%) was noticed when creating a model with one site's data and testing it against data from the remaining two sites (table $\mathbf{2}$ ).

Table 2. Correct classification rate for human breast tissue using models based on one site's data.

\begin{tabular}{|c|c|c|c|}
\hline $\begin{array}{c}\text { Model used for } \\
\text { training }\end{array}$ & $\begin{array}{c}\text { Correct classification rate } \\
\text { (\%) }\end{array}$ & $\begin{array}{c}\text { Rate of false } \\
\text { negatives (\%) }\end{array}$ & $\begin{array}{c}\text { Rate of false } \\
\text { positives (\%) }\end{array}$ \\
\hline C1 & 98.0 & 1.9 & 3.0 \\
\hline C3 & 100.0 & 0 & 0 \\
\hline C4 & 91.8 & 21.1 & 0 \\
\hline
\end{tabular}

For the cross-validation by site, a model where the training set contained the data of 2 sites was created and the data from the third site based were classified on this 2 site-model; all sites were left out once. This resulted in an overall $97.11 \%$ correct classification rate with $1.4 \%$ false negative and $5 \%$ false positive rates (table 3 ). A leave-one-patient-out crossvalidation (keeping all data in the training set but one patient) resulted in $98.57 \%$ correct classification rate with a $2.8 \%$ false negative and $0.72 \%$ false positive rate (table 3 ). 
a)

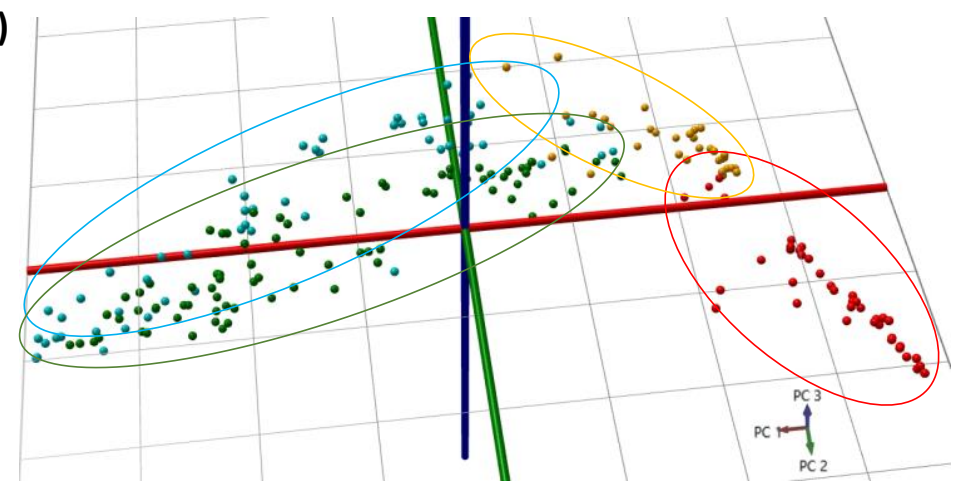

b)

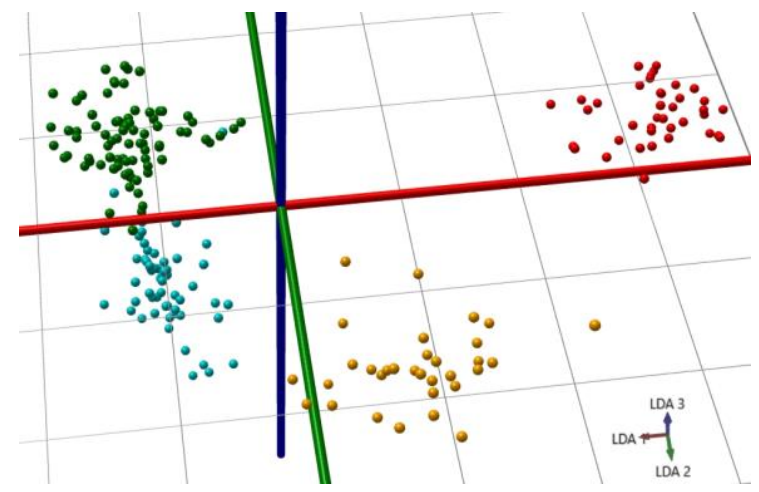

- normal breast - cut

- normal breast - coag

- breast tumor - cut

- breast tumor - coag

c)

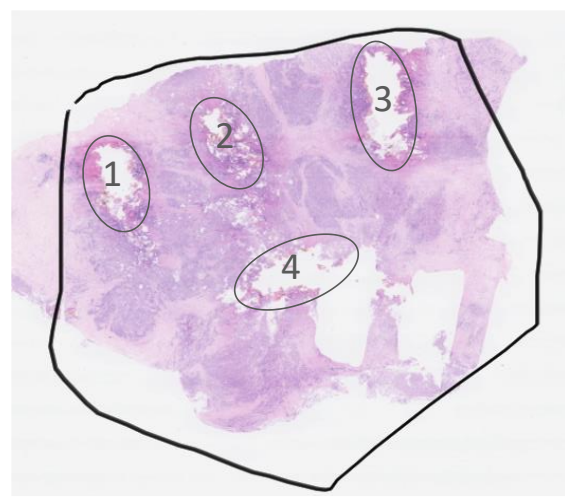

Figure 14. Multivariate analysis of REIMS spectra acquired from human breast tissue at each site. Each centre analysed human breast tissue samples by REIMS using both cut and coagulation mode. The PCA (a) and PCA/LDA (b) score plots compare the overall tissue-type variability between the two tissue sampling modes. (c) Representative H\&E stained section obtained from site C1. Burnt area numbered from 1-4 were assigned by a pathologist and used for labelling the spectra presented in (a) and (b). The surrounded area highlights a region containing $90 \%$ invasive breast cancer. 
Table 3. Confusion matrix for human breast tissue based on one-site-out, or one-patient-out cross validation.

\begin{tabular}{|c|c|c|c|c|c|c|}
\hline \multirow[b]{2}{*}{$\begin{array}{l}\text { True Class } \\
\downarrow\end{array}$} & \multicolumn{3}{|c|}{ Predicted Class (No. of spectra) } & \multirow{2}{*}{$\begin{array}{c}\text { Correct } \\
\text { classification } \\
\text { rate (\%) }\end{array}$} & \multirow{2}{*}{$\begin{array}{l}\text { Rate of false } \\
\text { negatives (\%) }\end{array}$} & \multirow{2}{*}{$\begin{array}{l}\text { Rate of false } \\
\text { positives (\%) }\end{array}$} \\
\hline & Tumour & Normal & Total & & & \\
\hline Tumour & $70^{1}$ & $1^{1}$ & 71 & \multirow{3}{*}{97.1} & \multirow{3}{*}{1.4} & \multirow{3}{*}{5.0} \\
\hline Normal & $7^{1}$ & $132^{1}$ & 139 & & & \\
\hline Total & 77 & 133 & 210 & & & \\
\hline Tumour & $69^{2}$ & $2^{2}$ & 71 & \multirow{3}{*}{98.6} & \multirow{3}{*}{2.8} & \multirow{3}{*}{0.7} \\
\hline Normal & $1^{2}$ & $138^{2}$ & 139 & & & \\
\hline Total & 70 & 140 & 210 & & & \\
\hline
\end{tabular}

\section{Discussion}

An international consortium to study the robustness of REIMS technology was formed across 4 sites, and multivariate models created at each site were tested for the purpose of evaluating the potential clinical utility of REIMS. Using identical equipment, it was found that variability in REIMS spectra can be minimized by harmonizing sampling methods and instrument settings. A combination of factors are likely to account for the range of correct classification rates (69-100\%) observed for food-grade meat among site-specific models such as: differences in meat sources, relative variance in the testing data set compared to the validation data set, and/or composition in major phospholipid peaks, which appeared to vary by site. The importance of variance in PCA/LDA-based classifiers of tissue was demonstrated in recent study that used both diathermy ( $C V=14-23 \%)$ and $\mathrm{CO}_{2}$ laser $(\mathrm{CV}=$ 9-12\%) to study an identical series of tissue samples (306). Using a diathermy-based model (larger variance) to classify the laser data gave an accuracy of 58\%. Conversely, a model based on the more precise laser data tested against diathermy halved the accuracy; but the accuracy could be restored to $58 \%$ if tissue-specific peak lists were used for model creation and testing. This work suggests that the impact of variance on misclassifications could be minimized by using a more targeted approach. Among the study's diathermy-based models, the combination of a more precise model and being the only non-EU meat source, may have contributed to the poor accuracy performance of C4's model, even though patterns acquired at $\mathrm{C} 4$ were accurately classified by other models.

To study the clinical applicability of REIMS, human breast tissues were analysed ex vivo across 3 sites. Striking differences in spectra between pathology-validated invasive breast cancer and normal breast adipose were characterized by altered relative abundance of phospholipid versus triglycerides. This overall pattern was consistent in most samples and the single-site models provided a correct classification rate with accuracy greater than 92\%; suggesting that the models were not over-fit. Nevertheless, combining two site's data into a single model and performing one-site-out cross validation gave an accuracy of at least $97 \%$, improving the sensitivity over the single-site models overall. To conclude, the phospholipid/triglyceride $\mathrm{m} / \mathrm{z}$ range is an informative signature for recognizing invasive 
breast cancer across sites, which is consistent with a much larger single-site cohort study published by C1 in 2017 (305). A larger study will be needed to identify sub-patterns that are correlated to receptor status, ductal carcinoma in-situ, or other relevant non-malignant conditions of the breast across sites. At the time of sample collection and gross assessment by pathology, it was not anticipated that $40 \%$ of the invasive cancer spectra would need to be excluded due to low amount of vital tumour cells in the sample as determined by histopathology; thus it is recommended that future multi-site studies involving REIMS overestimate the number of planned tumour samples.

Emerging clinical utility of mass spectrometry profiling has warranted evaluation of the performance of these techniques over multiple sites and patient groups. Targeted mass spectrometry assays based on quantitative isotope-dilution methods are typically evaluated on the basis variation of specific analyte quantities over time, and in comparison to a reference material or method. However, non-quantitative, broad-based patterns acquired by REIMS and related techniques, need to be evaluated based on accuracy of pattern recognition algorithms in comparison to a 'gold standard' technique such as histopathology. With data collected using the MasSpec Pen coupled to a high-mass resolution instrument, Sans and colleagues studied the performance of LASSO models based on both small molecules and lipids to detect ovarian cancer using 3 batches of samples from the same tissue banks over 2015-2018. The group found that when combining data from 2 batches to create a training group, the accuracy for high-grade tumours improved from 90 to $100 \%$ in the validation group, compared to using only one batch of data in the training group (307). Furthermore, the study demonstrated high accuracy of the model when using a lower-cost, low mass resolution instrument that may be more feasible to implement into clinical workflows (307). The same group performed a 2-site study using normal versus invasive breast cancer samples from Europe, United States, Asia and Brazil, this time using mass spectral patterns acquired by DESI in combination with LASSO to create inter-laboratory training and testing cohorts (308). Consistent with the conclusion of the present study using REIMS, the group found that normal breast and invasive breast cancers are similar enough among countries and races; based on phospholipids but also small molecules and FAs. Inter-laboratory comparison however, was based only on the phospholipid range due to between-site variability in features with $\mathrm{m} / \mathrm{z}<700$ proposed to arise from a combination of lipid degradation, sample handling/transportation and small differences in DESI sprayer geometry between laboratories (308). Other multi-site studies involving MALDI mass spectrometry imaging have also been reported, and explore sitedependent variability in factors such as protein vs. metabolite imaging (309), trypsin application and digestion (310), and biomarker discovery (83). As compared with other multisite studies involving ambient ionization mass spectrometry, the approach of the present study included a greater number of unique sites and instruments operated by different analysts; as well as independently sourced samples from each site. Most importantly, clinical samples were sourced from individual patients who underwent standard-of-care surgical treatment of invasive breast cancer at each site involving local pathology assessment. 


\section{Conclusion}

Taken together, the results of this study reveal that REIMS can differentiate with high accuracy normal breast tissue from invasive breast cancer in clinical samples independently collected, analysed and validated in UK, Europe and Canada. The data and others show that models which maximize the number of data points used from the broadest cross-section of patients and regions possible, will lead to a more accurate classification of tissue types. The results, in addition to the establishment of reference material and standard operating procedures demonstrate that accurate intraoperative classification of breast tissue by REIMS is possible. All of the sites have developed clinical workflows for use of mobile REIMS units in the operating theatre, enabling to test this hypothesis. 
Chapter 4

\section{Stromal vapours for real-time molecular guidance of breast-conserving surgery}




\begin{abstract}
Achieving radical tumour resection while preserving disease-free tissue during breastconserving surgery (BCS) remains a challenge. Here, mass spectrometry technologies were used to discriminate stromal tissues reported to be altered surrounding breast tumours, and build tissue classifiers ex vivo. Additionally, we employed the approach for in vivo and real-time classification of breast pathology based on electrosurgical vapours. Breastresected samples were obtained from patients undergoing surgery at MUMC+. The specimens were subsequently sampled ex vivo to generate electrosurgical vapours analysed by rapid evaporative ionization mass spectrometry (REIMS). Tissues were processed for histopathology to assign tissue components to the mass spectral patterns.

A total of 689 ex vivo REIMS patterns were collected from 72 patients which were analysed using multivariate statistical analysis (PCA-LDA). These patterns were classified as adipose, stromal and tumour tissues with $92.3 \%$ accuracy with a leave-one-patient-out crossvalidation. Tissue recognition using this ex vivo-built REIMS classification model was subsequently tested in vivo on electrosurgical vapours. Stromal and adipose tissues were classified during one BCS. Complementary ex vivo analyses were performed by REIMS and by desorption electrospray ionization mass spectrometry (DESI-MS) to study the potential of breast stroma to guide BCS. Tumour border stroma (TBS) and remote tumour stroma (RTS) were classified by REIMS and DESI-MS with $86.4 \%$ and $87.8 \%$ accuracy, respectively.
\end{abstract}

This study supports that stromal molecular alterations surrounding breast carcinomas can guide BCS in real-time using REIMS analysis of electrosurgical vapours.

\title{
Introduction
}

More than one out of ten women worldwide will be diagnosed with breast cancer during her life. The medical journey of patients with breast cancer has improved substantially over the years with broader screening, molecular and genomic diagnosis (14), and increased targeted therapeutic options (15). The life expectancy of patients with breast cancer has risen substantially, and the quality of life of breast cancer survivors has risen as a focus consideration for clinical decisions. The objective for the patient goes beyond the cure but includes the reduction of adverse effects and the improvement of cosmetic and sensitive reparation (311,312).

Breast conserving surgery (BCS) is a broadly used surgical treatment for patients with earlystage breast cancer and consists of achieving full tumour removal while conserving as much as possible healthy tissues. Despite assistance options with intraoperative diagnostic techniques such as ultrasound or radioactive seed localization (12), BCS can remain a challenge for the surgeon. In the current setting, the outcome of the operation can only be determined in detail after the pathology examination of the resected tissue. An unsuccessful outcome can lead to reoperation or more burdensome adjuvant treatments for the patient (299). 
Despite its soft structure, recent advances in ambient mass spectrometry have enabled the analysis of breast tissues $(305,308,313,314)$ by desorption electrospray ionization mass spectrometry imaging (DESI-MSI) (28) and rapid evaporative ionization mass spectrometry (REIMS) $(29,315)$ to predict histopathology based on metabolic patterns. While DESI-MSI uses charged droplets to desorb molecules from tissue sections to generate in situ two dimensional molecular distributions with precise histopathology examination, REIMS analyses electrosurgical vapours of tissue slices ex vivo and in vivo during surgery. After building a library of histologically validated lipid patterns with REIMS, tissues can be classified within seconds, which matches with the intraoperative need for pathological feedback $(29,308,315)$. Adapted to routine surgical tools, REIMS advantage is its potential implementation without changing the operation workflow and providing an on-line feedback about tissue pathology resected by the surgeon based on the chemical information present in the vapours produced in vivo.

DESI has determined specific metabolic patterns for adipose, stromal, glandular and tumour of breast tissues $(313,314)$ but REIMS has discriminated normal breast and breast tumour tissues only (305). The variety of healthy breast tissues reported with DESI-MSI suggests that specific metabolic patterns for different healthy breast tissues could be expected with REIMS, which remains unexploited. Moreover, alterations of stromal tissues surrounding breast tumours have been associated to clinical observations $(316,317)$. If detectable in real-time, these stromal changes could mark a safe area of resection to complement the assessment of margin of resection by histopathology. They would constitute a classification of healthy and tumour surrounding tissues for precise surgical guidance.

Herein, the potential of breast stroma to guide BCS is investigated by mass spectrometry analysis. First, a classification model is built based on REIMS analysis of ex vivo electrosurgical vapours to recognize breast stroma during BCS. Then, REIMS and DESI-MS are used to investigate further the potential of breast stroma to guide BCS.

\section{Materials and methods}

\section{Study population and tissue procuration}

The study included 85 female patients (table 1) undergoing surgery for breast tumour at Maastricht University Medical Centre (MUMC+) between September 2017 and September 2019. Patient inclusion followed a protocol approved by the Medical Ethics Committee (Medisch-Ethische ToetsingsCommissie) azM/UM of MUMC+ (approval number METC 164-168). The study was conducted with highest practice standards according to the revised version of the Declaration of Helsinki. Written informed consent was obtained from each patient prior to study participation. A pathologist selected samples on the resected-tissue for the present study; samples were taken of the tumour and benign tissue a few centimetres away from the tumour. Tissue slices were used for REIMS ex vivo analysis, or frozen in liquid nitrogen and stored at $-80^{\circ} \mathrm{C}$ until DESI analysis. 


\section{REIMS ex vivo analysis}

Tissues were cauterized ex vivo using a monopolar hand-piece (iKnife disposable device, Waters, Hungary) equipped with a $1.7 \mathrm{~cm}$-diameter blade electrode, connected to an electrosurgical heat-generator (Force FX, Covidien Ltd.), operated in cut modality. The generated vapours were aspirated into a mobile REIMS Xevo G2-XS Q-ToF mass analyser (Waters Corporation, Wilmslow, UK). Isopropanol (Biosolv or Honeywell) containing leucine-encephalin (Sigma-Aldrich) was infused at $150 \mu \mathrm{L} / \mathrm{min}$ for lock-mass correction (318). REIMS acquisitions were performed in negative ionization mode over the mass range $\mathrm{m} / \mathrm{z} 100$ to 1500 . After REIMS analysis, the remaining tissue was formalin fixed and paraffin embedded. Tissue blocks were sectioned at $5 \mu \mathrm{m}$-thickness. Tissue sections were stained with haematoxylin and eosin (H\&E). A breast pathologist attributed tissue components that surround the sampling spots without knowledge of the MS patterns. Percentages of tissue components were assigned to each MS profile based on the histology surrounding of each sampling spot. For the classification of TBS and RTS, only the stroma patterns correctly classified in the adipose/tumour/stroma REIMS model were included. Stromal patterns generated on tissue sampled at least a few centimetres from the tumour and presenting no tumour were considered RTS patterns. Stroma patterns generated on tissues containing sampling spots containing a tumour component in their pathology examination were considered TBS patterns.

\section{REIMS in vivo analysis}

In vivo measurement was performed on the same instrument. Surgery was performed using commercial hand-piece (Erbe) and heat generator (Valleylab FT10, Covidien Ltd.). Three GoPros (Hero4) were set up to record surgical site, heat generator and screen of the computer of the mass spectrometer to coordinate in time, site of electrosurgical vapours production, the diathermia parameters and MS profile generation. Conditions for experimental measurements were set up the same as for ex vivo analysis with air gas. Electrosurgical vapours were directed towards the mass spectrometer and the REIMS source and partially discarded as usual.

\section{DESI-MSI}

Two groups of specimens were selected for DESI-MSI analysis; samples of the tumour site or samples of fibro-glandular tissues away from the tumour site. Frozen tissues were sectioned using a cryotome (Microm) at $10 \mu \mathrm{m}$-thickness, thaw mounted on histological slides (Superfrost) and stored at $-80^{\circ} \mathrm{C}$ prior analysis. Experiments were performed on a Xevo G2-XS Q-ToF MS (Waters Corporation, Wilmslow, UK). 98\% methanol/water (Biosolv) at $1.5 \mu \mathrm{l} / \mathrm{min}$ was used as a solvent. Measurements used for the tissue classification were performed in negative ionization mode, over the mass range $\mathrm{m} / \mathrm{z} 100-1000$ at $40 \times 40 \mu \mathrm{m}^{2}$ pixel size, acquired at scan rate of $150 \mu \mathrm{m} / \mathrm{sec}$. Tissue sections were stained by H\&E and scanned on a slide scanner (Ventana) after DESI-MSI analysis. 
Table 1. Baseline characteristics of the patient population. IQR: interquartile range, BMI: body mass index

$\begin{array}{lcccc}\text { Studies } & \text { All combined } & \text { DESI-MS } & \text { REIMS ex vivo } & \text { REIMS in vivo } \\ \text { Number of patients } & 85 & 22 & 72 & 1 \\ \text { Clinical characteristics I } & & & & \\ \text { Age, years median, IQR } & 61(53-72) & 57(53-72) & 61(53-72) & 69 \\ \text { BMI, kg/m² median, IQR } & 25.8(23.7-29.2) & 25.6(24.3-28.8) & 26.2(24.1-29.4) & 23.1 \\ \text { N patients unknown BMI } & 9(11) & 3 & 9 & 0 \\ \text { Clinical characteristics II, number of patients }(\%) & & & \\ \text { Menopausal status } & & & & \\ \text { Premenopausal } & 18(21 \%) & 6(27 \%) & 14(19 \%) & \\ \text { Perimenopausal } & 4(5 \%) & 2(9 \%) & 3(4 \%) & \\ \text { Postmenopausal } & 55(65 \%) & 12(55 \%) & 47(65 \%) & \\ \text { Unknown } & 8(9 \%) & 2(9 \%) & 8(11 \%) & \\ \text { Parity } & & & & \\ \text { P0 } & 13(15 \%) & 3(14 \%) & 11(15 \%) & \\ \text { P1 } & 9(11 \%) & 4(18 \%) & 7(10 \%) & \\ \text { P2 } & 35(41 \%) & 10(45 \%) & 29(40 \%) & \\ \text { P3 } & 15(18 \%) & 3(14 \%) & 13(18 \%) & \\ \text { P4 or more } & 3(4 \%) & 1(5 \%) & 2(3 \%) & \\ \text { Unknown } & 10(12 \%) & 1(5 \%) & 10(14 \%) & \\ \text { Tabaco use } & & & & \\ \text { Active or recent sevrage } & 18(21 \%) & 4(18 \%) & 16(22 \%) & \\ \text { Past or absent } & 61(72 \%) & 17(77 \%) & 52(72 \%) & \\ \text { Unknown } & 6(7 \%) & 1(5 \%) & 5(7 \%) & \\ \text { Patho } & & & \\ & & & \end{array}$

\begin{tabular}{|c|c|c|c|c|}
\hline \multicolumn{5}{|c|}{ Pathological characteristics, number of patients (\%) } \\
\hline \multicolumn{5}{|l|}{ Tumour stage } \\
\hline Tis & $4(5 \%)$ & $3(14 \%)$ & $2(3 \%)$ & \multirow{5}{*}{1} \\
\hline T0 & $3(4 \%)$ & $0(0 \%)$ & $3(4 \%)$ & \\
\hline $\mathrm{T} 1$ & $50(59 \%)$ & $13(59 \%)$ & $42(58 \%)$ & \\
\hline T2 & $25(29 \%)$ & $5(23 \%)$ & $23(32 \%)$ & \\
\hline T3/4 & $3(4 \%)$ & $1(5 \%)$ & $2(3 \%)$ & \\
\hline \multicolumn{5}{|l|}{ Tumour grade } \\
\hline Not applicable & $5(6)$ & $3(14 \%)$ & $3(4 \%)$ & \multirow{4}{*}{1} \\
\hline Grade I & $8(9)$ & $2(9 \%)$ & $7(10 \%)$ & \\
\hline Grade ॥ & $41(48)$ & $10(45 \%)$ & $33(46 \%)$ & \\
\hline Grade III & $31(36)$ & $7(32 \%)$ & $29(40 \%)$ & \\
\hline \multicolumn{5}{|c|}{ Lymph node status } \\
\hline $\mathrm{Nx}$ & $1(1 \%)$ & $0(0 \%)$ & $1(1 \%)$ & \multirow{4}{*}{1} \\
\hline No & $55(65 \%)$ & $15(68 \%)$ & $46(64 \%)$ & \\
\hline N1 & $22(26 \%)$ & $6(27 \%)$ & $18(25)$ & \\
\hline $\mathrm{N} 2 / 3$ & $9(11 \%)$ & $1(5 \%)$ & $7(10 \%)$ & \\
\hline
\end{tabular}

Treatment, number of patients (\%)

\section{Surgery}

BCS

Ablation

Neoadjuvant therapy

Yes

No
$28(33 \%)$

$57(67 \%)$

$12(14 \%)$

$73(86 \%)$
$3(14 \%)$

$19(86 \%)$

$3(14 \%)$

$19(86 \%)$
25 (35\%)

47 (65\%)

$10(14 \%)$

$62(86 \%)$

\section{1}

1 
Stromal areas surrounding remote normal glands and surrounding tumours were selected by a breast pathologist using QuPath (v0.1.2). One data point corresponded to the sum of the signal of 6 adjacent pixels selected in TBS or RTS areas in HD Imaging (v1.5, Waters, UK). Experiment was performed over the mass range $\mathrm{m} / \mathrm{z} 50-2000$ at $30 \times 30 \mu \mathrm{m}^{2}$ pixel size at scan rate of $100 \mu \mathrm{m} / \mathrm{sec}$.

\section{Data analysis}

Data were analysed using a prototype of abstract model builder software (AMX v1.01563.0, Waters Corporation, Budapest, Hungary). PCA-LDA and leave-one-patient-out crossvalidation were used to compare the accuracy between the histopathology classification and the MS-based classification.

\section{Molecular identification}

Spectra were lock-mass corrected on deprotonated leucine-encephalin $m / z$ 554.2615 [M$\mathrm{H}^{-}$for REIMS and deprotonated raffinose $\mathrm{m} / \mathrm{z} 503.1606[\mathrm{M}-\mathrm{H}]^{-}$for DESI-MS for identification based on mass accuracy. Tandem mass spectrometry experiments were performed by collision-induced dissociation with argon gas. Experimental data were tested on ALEX ${ }^{123}$ lipid calculator (319) or compared to reference literature (314).

\section{Results}

Ex vivo built database enables in vivo tissue recognition

In total, 689 REIMS ex vivo patterns were generated from 72 patients and were attributed as 209 stroma, 256 adipose and 224 tumour patterns by histopathology (tables $\mathbf{2}$ and $\mathbf{3}$ ).

REIMS patterns were classified with $92.6 \%$ accuracy (fig. 1a-c). A PCA score plot (fig. 1a) showed a separation of adipose and tumour patterns along the PC1 axis (which describes $56.2 \%$ of the variance of the data) and a separation of stroma and tumour patterns along the PC2 axis (25.6\%). Main discriminators were $\mathrm{m} / \mathrm{z} 893.75$ and $\mathrm{m} / \mathrm{z} 919.75$ (fig. 1d) previously assigned as triglycerides (305) for adipose, and $\mathrm{m} / \mathrm{z} 255.25$ and $\mathrm{m} / \mathrm{z} 281.25$ (fig. 1e) assigned as fatty acids, palmitic acid and oleic acid respectively, for stroma (table 4). Single mass patterns for each tissue type are shown in figure $\mathbf{1} \mathbf{f}-\mathbf{h}$.

Table 2. Pathological characteristics of the tumour patterns included in the library for the REIMS ex vivo study. N.A.: unknown or not applicable, DCIS: ductal carcinoma in situ, IDC: invasive ductal carcinoma, IDLC: invasive ducto-lobular carcinoma, ILC: invasive lobular carcinoma, ER: oestrogen receptor, PR: progesterone receptor: HER2: human epidermal growth factor receptor 2

\begin{tabular}{l|cccccccccccccccc|} 
& \multicolumn{3}{c}{ Tumour type } & \multicolumn{3}{c}{ ER/PR status } & \multicolumn{3}{c}{ HER2 status } & \multicolumn{3}{c}{ Tumour grade } \\
& DCIS & IDC & IDLC & ILC & ER+/PR+ ER+/PR- ER-/PR- N.A. & HER2+ & HER2- N.A. & I & II & III & N.A. \\
\hline Patterns & 3 & 176 & 1 & 44 & 158 & 30 & 28 & 8 & 46 & 171 & 7 & 9 & 76 & 136 & 3 \\
Patients & 2 & 35 & 1 & 12 & 34 & 8 & 5 & 3 & 6 & 41 & 3 & 3 & 21 & 24 & 2
\end{tabular}


Table 3. Number of REIMS patterns per tissue type and per patient included in the library for the REIMS ex vivo study. P: patient, A: adipose, T: tumour, S: stroma, RTS: remote tumour stroma, TBS: tumour border stroma

\begin{tabular}{|c|c|c|c|c|c|c|}
\hline REIMS & A & T & S & RTS & TBS & TOTAL \\
\hline P1 & 1 & 8 & & & & 9 \\
\hline P2 & 2 & 8 & 5 & 5 & & 15 \\
\hline P3 & 2 & 5 & 2 & & 1 & 9 \\
\hline P4 & 6 & 2 & & & & 8 \\
\hline P5 & 5 & 2 & 4 & 2 & 2 & 11 \\
\hline P6 & 2 & 2 & 2 & & 2 & 6 \\
\hline P7 & & 1 & 4 & & 4 & 5 \\
\hline P8 & 2 & 7 & 4 & & 4 & 13 \\
\hline P9 & & 9 & 11 & 10 & 1 & 20 \\
\hline P10 & 2 & 16 & 7 & & 7 & 25 \\
\hline P11 & & 7 & & & & 7 \\
\hline P12 & 6 & 4 & 5 & 4 & & 15 \\
\hline P13 & 3 & 1 & & & & 4 \\
\hline P14 & 1 & 1 & & & & 2 \\
\hline P15 & 10 & & & & & 10 \\
\hline P16 & 3 & 7 & 1 & & & 11 \\
\hline P17 & & 6 & 5 & 4 & 1 & 11 \\
\hline P18 & 1 & & 6 & 6 & & 7 \\
\hline P19 & & 5 & 6 & 3 & 3 & 11 \\
\hline P20 & 2 & 5 & & & & 7 \\
\hline P21 & 2 & 2 & & & & 4 \\
\hline P22 & 6 & 15 & & & & 21 \\
\hline P23 & & 4 & 5 & 5 & & 9 \\
\hline P24 & 1 & & 8 & 4 & 3 & 9 \\
\hline P25 & 4 & 3 & 1 & & 1 & 8 \\
\hline P26 & 2 & & 6 & 4 & & 8 \\
\hline P27 & 1 & 2 & & & & 3 \\
\hline P28 & & & 7 & 7 & & 7 \\
\hline P29 & 10 & 2 & & & & 12 \\
\hline P30 & 2 & & 6 & 6 & & 8 \\
\hline P31 & 2 & 9 & 1 & & & 12 \\
\hline P32 & & 4 & & & & 4 \\
\hline P33 & & 1 & 3 & 2 & 1 & 4 \\
\hline P34 & & 3 & & & & 3 \\
\hline P35 & & & 1 & & 1 & 1 \\
\hline P36 & & & 6 & 3 & & 6 \\
\hline
\end{tabular}

\begin{tabular}{|c|c|c|c|c|c|c|}
\hline REIMS & A & T & S & RTS & TBS & TOTAL \\
\hline P37 & 1 & 6 & 5 & 4 & & 12 \\
\hline P38 & 2 & 1 & & & & 3 \\
\hline P39 & & 4 & 3 & 3 & & 7 \\
\hline P40 & & & 5 & 3 & 2 & 5 \\
\hline P41 & 1 & 16 & & & & 17 \\
\hline P42 & 2 & 4 & 14 & 13 & & 20 \\
\hline P43 & & 1 & & & & 1 \\
\hline P44 & & & 10 & 10 & & 10 \\
\hline P45 & 1 & & & & & 1 \\
\hline P46 & 6 & & 2 & 2 & & 8 \\
\hline P47 & 3 & & 3 & 3 & & 6 \\
\hline P48 & 3 & 1 & & & & 4 \\
\hline P49 & 16 & 1 & 6 & 4 & & 23 \\
\hline P50 & 5 & 3 & 10 & 9 & 1 & 18 \\
\hline P51 & 22 & 5 & 1 & & 1 & 28 \\
\hline P52 & 14 & & 4 & 1 & & 18 \\
\hline P53 & 7 & & 1 & & 1 & 8 \\
\hline P54 & 1 & 6 & & & & 7 \\
\hline P55 & 1 & 2 & 5 & 5 & & 8 \\
\hline P56 & 13 & 5 & & & & 18 \\
\hline P57 & 5 & & 2 & 1 & & 7 \\
\hline P58 & 5 & & 1 & & & 6 \\
\hline P59 & 4 & 7 & 2 & 1 & & 13 \\
\hline P60 & & & 4 & & 3 & 4 \\
\hline P61 & 13 & 1 & & & & 14 \\
\hline P62 & 3 & 2 & 10 & 9 & & 15 \\
\hline P63 & 9 & 2 & 5 & 2 & 3 & 16 \\
\hline P64 & 3 & 3 & 3 & 3 & & 9 \\
\hline P65 & 12 & & & & & 12 \\
\hline P66 & 4 & & & & & 4 \\
\hline P67 & 4 & 5 & 1 & & & 10 \\
\hline P68 & 3 & 2 & & & & 5 \\
\hline P69 & 6 & & & & & 6 \\
\hline P70 & 3 & & 4 & 2 & 1 & 7 \\
\hline P71 & 3 & 5 & 1 & & 1 & 9 \\
\hline & 3 & 1 & 1 & & 1 & 5 \\
\hline
\end{tabular}

Table 4. Molecular identification by REIMS based on mass accuracy.

\begin{tabular}{|lccccccc}
\hline Identification & Adduct & Formula & PCA $\mathbf{m} / \mathbf{z}$ & Measured $\mathbf{m} / \mathbf{z}$ & Theoretical $\mathbf{m} / \mathbf{z}$ & Mass error (ppm) \\
\hline Fatty acid 16:0 & $-\mathrm{H}^{+}$ & $\mathrm{C}_{16} \mathrm{H}_{31} \mathrm{O}_{2}$ & 255.25 & 255.23 & 255.23 & 1.4 \\
\hline Fatty acid 18:0 & $-\mathrm{H}^{+}$ & $\mathrm{C}_{18} \mathrm{H}_{35} \mathrm{O}_{2}$ & 281.25 & 281.25 & 283.26 & 0.7
\end{tabular}


a)

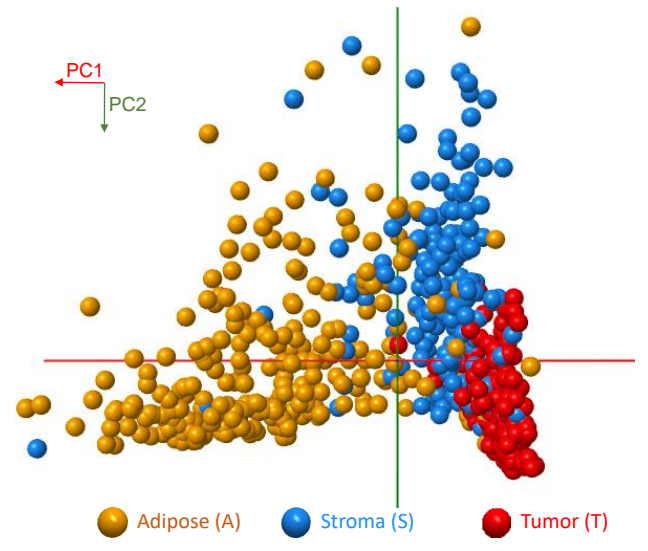

b)

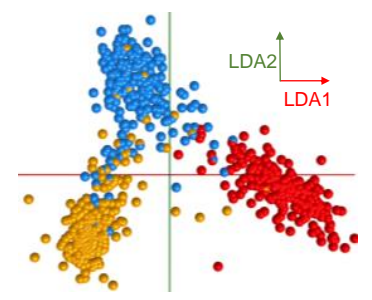

c)

\begin{tabular}{|c|c|c|c|c|}
\hline \multirow{2}{*}{\multicolumn{2}{|c|}{$\begin{array}{c}\text { Overall class rat } \\
92.3 \%\end{array}$}} & \multicolumn{3}{|c|}{ Predicted Class } \\
\hline & & A & $s$ & \\
\hline \multirow{3}{*}{$\begin{array}{l}\text { Actual } \\
\text { Class }\end{array}$} & A & $91.9 \%$ & $6.9 \%$ & $1.2 \%$ \\
\hline & s & $8.1 \%$ & $88.5 \%$ & $3.3 \%$ \\
\hline & $T^{T}$ & $0.4 \%$ & $3.5 \%$ & 6. \\
\hline
\end{tabular}

d)

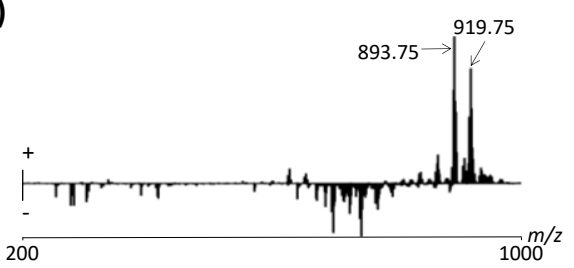

e)

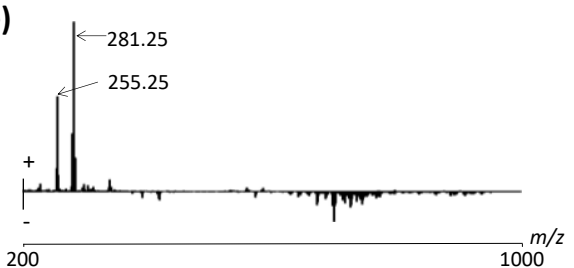

f)

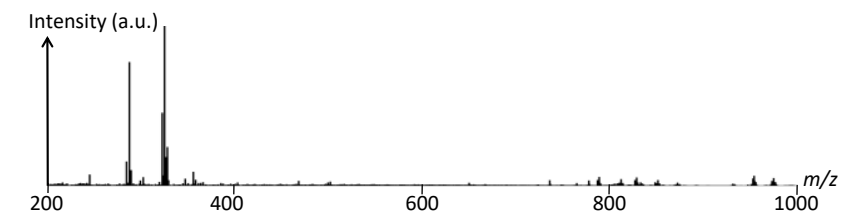

g) Intensity (a.u.)

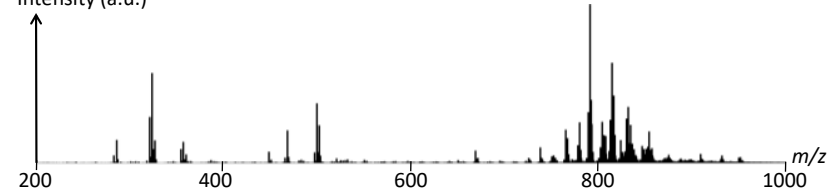

h) Intensity (a.u.)

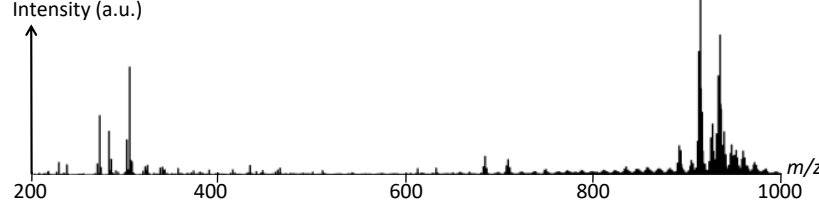

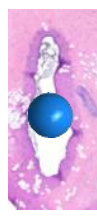<smiles>CCCCCC(C)=O</smiles>
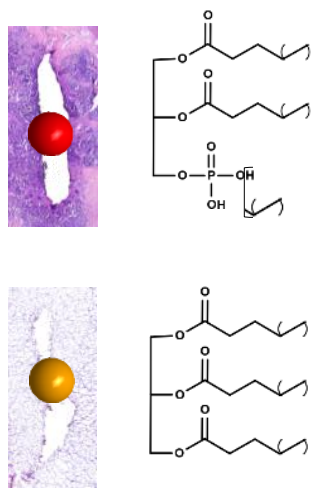

Figure 1. REIMS analysis of electrosurgical vapours ex vivo classifies tumour, stroma and adipose tissues. (a) PCA score plot (mass range $m / z$ 200-1000, PC1 describing 56.2\% of total variance, PC2 25.6\%). (b) Pseudo-LDA score plot. (c) Confusion matrix. (d-e) mass features loading plots for PC1 and PC2 respectively with indication of the two most discriminative mass features for adipose and stroma. (f-h) Mass spectral patterns generated from tissues examined as stroma, tumour and adipose tissues respectively with corresponding histology surrounding the sampling spot and general backbone structure of the main lipid class involved in the discrimination of the respective tissue type (based on our investigation and literature (305)). 
Next, in vivo REIMS data were collected in an operating room during one BCS performed using a diathermia knife. Intense MS signals were detected throughout the operation (fig. 2b). At around 14 minutes of recording, scan 845 and scan 851 (fig. 2b,e), both displayed signals generated in cut modality. The pictures taken during surgery indicate a change in the transected tissue (fig. 2c,f). While the most intense mass peaks in scan 845 were detected only in the mass range $\mathrm{m} / \mathrm{z} 200-400$, intense mass peaks were displayed in the mass range $m / z 800-1000$ for scan 851 .

The REIMS model of ex vivo patterns enabled the recognition of stroma on scan 845 and of adipose on scan 851 . Based on pathology report, stroma and adipose tissues were present on the resection margins on the mediodorsal orientation of the specimen (fig. $\mathbf{2} \mathbf{d}, \mathbf{g}$ ). These observations correlated with the signal measured in scans 845 and 851 . This demonstrates the potential of the REIMS classification model generated ex vivo to recognize breast tissue types in vivo during surgery.

a)

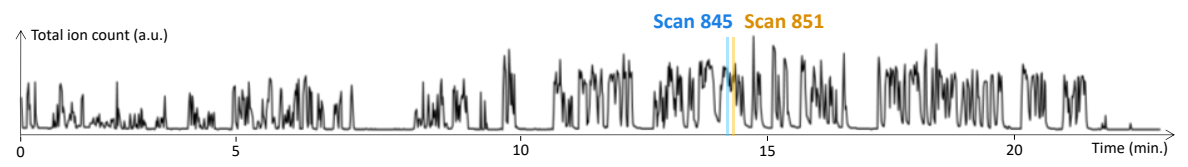

b)

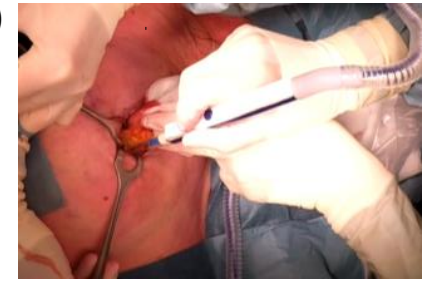

e)

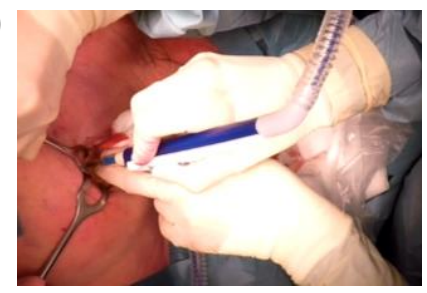

c)

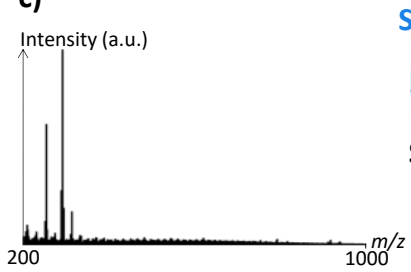

f)

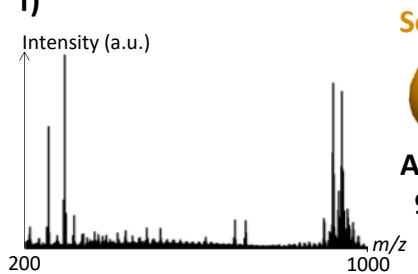

Scan 845

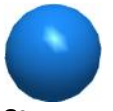

Stroma

$98.1 \%$

Scan 851

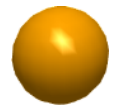

Adipose

$96.8 \%$ d)

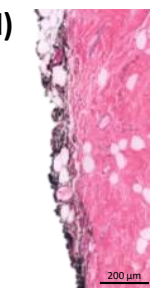

g)

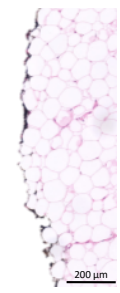

Figure 2. REIMS analysis of electrosurgical vapours enables in vivo tissue recognition. (a) Total ion count as a function of time analysed by REIMS of electrosurgical vapours in vivo during a BCS. (b,e) Surgical site, (c,f) mass pattern signals (lock-mass removed), scan number, tissue classification results and $(\mathbf{d}, \mathbf{g})$ probable histology resection margin based on the specimen orientation for scans 845 and 851 respectively.

\section{Stroma changes surrounding breast tumours}

Further investigations were pursued on 189 ex vivo REIMS patterns generated from tissues of 43 patients and designed as 44 TBS patterns and as 140 RTS patterns (table 3, fig. 3a). PCA score plot did not enable separation of TBS and RTS patterns, therefore no straightforward screening and molecular identification of the main mass peaks discriminant of TBS and RTS was possible. However, LCA score plot enabled a good separation (fig. 3c) and mass patterns were classified as TBS or RTS with $86.4 \%$ accuracy (fig. 3d). 
a)
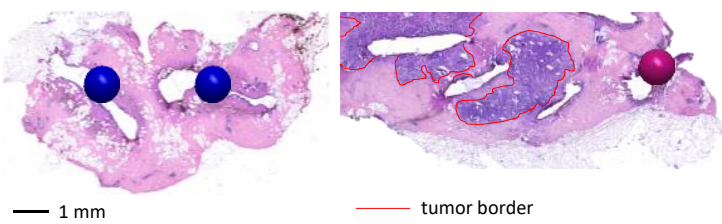

b)

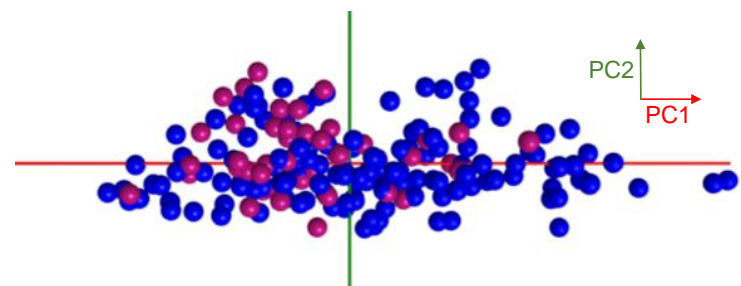

c)

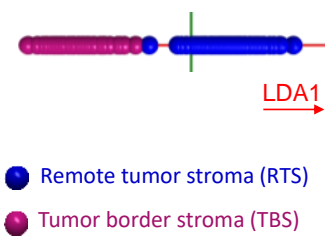

d)

\begin{tabular}{|c|c|c|c|}
\hline \multirow{2}{*}{\multicolumn{2}{|c|}{$\begin{array}{c}\text { Overall class rate } \\
86.4 \%\end{array}$}} & \multicolumn{2}{|c|}{ Predicted Class } \\
\hline & & REIMS & REIMS \\
\hline \multirow{2}{*}{$\begin{array}{l}\text { Actual } \\
\text { Class }\end{array}$} & $\begin{array}{l}\text { REIMS } \\
\text { RTS }\end{array}$ & $89.3 \%$ & $10.7 \%$ \\
\hline & $\begin{array}{l}\text { REIMS } \\
\text { TBS }\end{array}$ & $22.2 \%$ & $77.8 \%$ \\
\hline
\end{tabular}

Figure 3. REIMS patterns discriminate tumour border stroma (TBS) and tumour remote stroma (TRS). (a) Histological sampling spots examined as RTS and TBS. (b) PCA score plot (mass range $\mathrm{m} / z$ 200-500, PC1 describing $82.6 \%$ of total variance, PC2 $7.3 \%$ ). (c) LDA score plot. (d) Confusion matrix.

REIMS experiments were complemented with DESI experiments for more precise analysis of the stromal changes surrounding tumour borders. In total, 196 DESI-MS patterns, including 98 remote tumour stroma patterns (RTS) and 98 tumour border stroma (TBS) patterns, were extracted from pixels of 22 tissue sections from 22 patients (table 5). PCA score plot displayed a separation RTS and TBS mainly along the PC1 axis (fig. 4a). DESI-MS patterns were classified as TBS or RTS with $87.8 \%$ accuracy (fig. $\mathbf{4 b}$,d). Main discriminator of TBS along PC1 mass loading plot was a mass feature $\mathrm{m} / \mathrm{z} 201.05$ (fig. 4c) corresponding to mass value $m / z 201.04$ previously identified (15) and confirmed (table 6) as lactate dimer. Its distribution delineates tumour borders with intense signal in the TBS areas (fig. 4e).

Table 5. Number of REIMS patterns per tissue type and per patient included in the library for the DESI-MS study to classify tumour border stroma (TBS) and tumour remote stroma (TRS). P: patient.

\begin{tabular}{|c|c|c|c|}
\hline DESI-MS & RTS & TBS & TOTAL \\
\hline P74 & 9 & & 9 \\
P75 & 9 & & 9 \\
P10* & & 6 & 6 \\
P12* & 5 & & 5 \\
P76 & & 9 & 9 \\
P17* & 9 & & 9 \\
P30* & 12 & & 12 \\
P31* & & 8 & 8 \\
P33* & & 9 & 9 \\
P77 & 9 & & 9 \\
P78 & & 10 & 10 \\
\hline *contributed also to REIMS study
\end{tabular}

\begin{tabular}{|c|c|c|c|}
\hline DESI-MS & RTS & TBS & TOTAL \\
\hline P49* & & 9 & 9 \\
\hline P79 & 9 & & 9 \\
\hline P55* & 9 & & 9 \\
\hline P80 & & 8 & 8 \\
\hline P62* & & 9 & 9 \\
\hline P64* & 9 & & 9 \\
\hline P81 & & 9 & 9 \\
\hline P82 & & 9 & 9 \\
\hline P83 & & 12 & 12 \\
\hline P84 & 9 & & 9 \\
\hline P85 & 9 & & 9 \\
\hline TOTAL & 98 & 98 & 196 \\
\hline
\end{tabular}

Table 6. Lactate dimer identification by DESI-MS based on mass accuracy and tandem MS.

\begin{tabular}{|cccccccc} 
Identification & Adduct & Formula & $\begin{array}{c}\text { PCA } \\
\mathbf{m} / \mathbf{z}\end{array}$ & $\begin{array}{c}\text { Measured } \\
\mathbf{m} / \mathbf{z}\end{array}$ & $\begin{array}{c}\text { Theoretical } \\
\mathbf{m} / \mathbf{z}\end{array}$ & $\begin{array}{c}\text { Mass error } \\
\text { (ppm) }\end{array}$ & $\begin{array}{c}\text { Fragment ion } \\
\text { upon } \mathbf{M S} / \mathrm{MS}\end{array}$ \\
\hline Lactate dimer & {$[2 \mathrm{M}+\mathrm{Na}]^{-}$} & $\mathrm{C}_{6} \mathrm{H}_{10} \mathrm{O}_{6} \mathrm{Na}$ & 201.05 & 201.04 & 201.04 & 4 & 89.0
\end{tabular}


a)

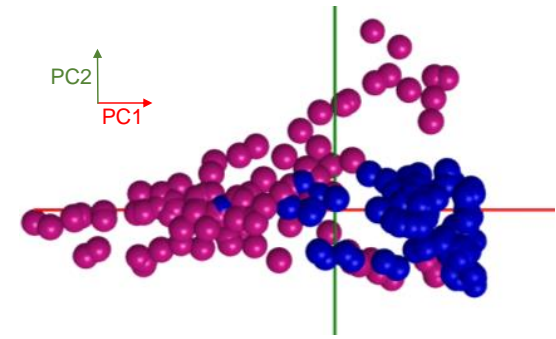

b)

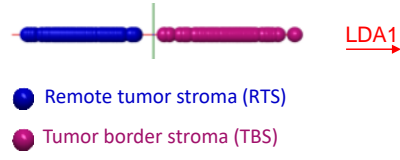

c)

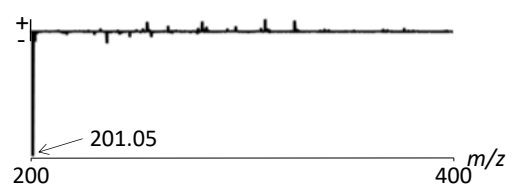

d)

\begin{tabular}{|c|c|c|c|}
\hline \multirow{2}{*}{\multicolumn{2}{|c|}{$\begin{array}{c}\text { Overall class rate } \\
87.8 \%\end{array}$}} & \multicolumn{2}{|c|}{ Predicted Class } \\
\hline & & $\begin{array}{l}\text { DESI-MS } \\
\text { RTS }\end{array}$ & $\begin{array}{l}\text { DESI-MS } \\
\text { TRS }\end{array}$ \\
\hline \multirow{2}{*}{$\begin{array}{c}\text { Actual } \\
\text { Class }\end{array}$} & $\begin{array}{c}\text { DESI-MS } \\
\text { RTS }\end{array}$ & $83.7 \%$ & $16.3 \%$ \\
\hline & $\begin{array}{c}\text { DESI-MS } \\
\text { TBS }\end{array}$ & $8.1 \%$ & $91.8 \%$ \\
\hline
\end{tabular}

e)

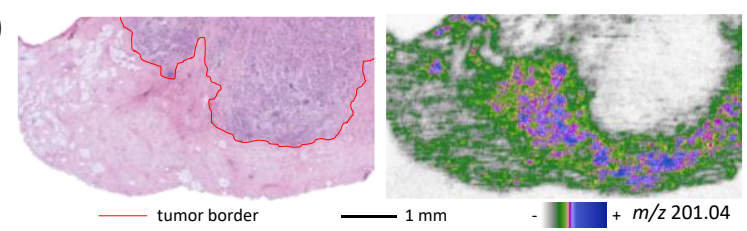

Figure 4. DESI-MS patterns discriminate tumour border stroma (TBS) and tumour remote stroma (TRS). (a) PCA score plot (mass range $\mathrm{m} / \mathrm{z}$ 200-400, PC1 describing 76.8\% of total variance, PC2 12.6\%). (b) LDA score plot. (c) PC1 loading plot with indication of the most discriminative mass feature for TBS. (d) Confusion matrix. (e) Histology and molecular distribution of lactate dimer $(\mathrm{m} / \mathrm{z} 201.04)$ in a histologically normal stroma surrounding an invasive ductal carcinoma.

\section{Discussion}

MSI for precise molecular breast pathology and its lipid patterns

The development MSI towards clinical applications went alongside the molecular characterization of breast pathology. The first mass spectrometry use for delineation of breast cancer margins used DESI-MSI to discriminate cancerous and glandular tissues (313). Fatty acids, including oleic acid, were reported more abundant in cancerous tissues than in normal glandular tissues. Moreover, they reported a separation based on the ER/PR status on tumour tissues from 14 patients. This finding was later confirmed (314) on 28 intraoperative tumour biopsies assessing the changes of tumours metabolic patterns as affected mainly by the grade and the hormone ER/PR receptor status rather than the tumour type. Metabolic patterns also evaluated the micro-invasion for the discrimination of in situ and invasive ductal carcinomas and molecular subtypes $(320,321)$. However, the classification of HER2 status was reported successful only on proteomic patterns (322).

The amplitude of the breast cancer research led to the establishment of two main reported MSI multicentre studies $(83,308)$ to validate the reproducibility for clinically relevant applications. Porcari et $a l$. (308) included their results in the continuity of the previous findings, especially the classification receptor status. Despite variation in parameters and instrumentations, investigations reported similar performances of the classification of breast tissues bringing MSI closer to the qualification as a reliable tool for diagnostics use (323). 


\section{REIMS lipid patterns of breast pathology}

This work with REIMS reported the ex vivo classification of adipose, stroma and tumour tissues mainly characterized by the increase of the intensities of mass peaks assigned as fatty acids, glycerophospholipids and triglycerides respectively. A connection can be hypothesized between the interpretation of these results and the heterogeneity of the cellular composition for each tissue type. As glycerophospholipids are known constituent of cellular membranes (324), the cellularity of the tumours makes its patterns characterized by intense glycerophospholipids. Proteins with structural support and connective functions such as collagen mainly composed the stroma. Therefore, the low number of cells, in general limited to a few fibroblasts in the stroma makes its REIMS patterns characterized by a relative increase of fatty acids. For adipose tissues, the intense signal of triglycerides goes along with the composition of its lipid vacuoles that affects the signal a lot more than membrane glycerophospholipids.

\section{Breast stroma and the molecular margin of resection}

This study confirms the changes of metabolic patterns between remote and tumour stromas on intraoperative breast biopsies by DESI-MSI (314). Metabolic alterations of the tumour microenvironment lay the bases of how insight in molecular changes could improve surgical precision.

Technological improvements of speed and intraoperative sampling $(30,31)$ recently contributed to emerging mass spectrometric tissue classifiers for clinical applications (27). Paradoxically, the molecular margin of resection (325), beyond histopathology, characterized in earlier studies to understand tumour recurrence $(212,326)$, was set aside of the most recent investigations. The benefit of mass spectrometric technologies for clinical applications has been mainly considered only by histopathological validation which remains the gold standard for disease-free patient survival. Nevertheless, the detection of more subtle molecular changes, not detectable by histopathology, could be even more valuable, as previously reported by REIMS on primary liver tumours (29). REIMS has been mainly reported in the perspective to correct the execution of unsafe resection margins by near real-time classification of tumour and normal tissues during surgery. This report suggests that surgeons could benefit from a critical information, the distance to the tumour, with recognition of breast stroma metabolic patterns during surgery. Beyond a correction, this would constitute a real-time molecular guidance to improve surgical precision of BCS. This is expected to be a major leap towards achieving the smallest possible resections with safe margins. The classification of breast pathology from electrosurgical vapours generated ex vivo enabled direct stroma recognition in vivo. This augments the value of REIMS for in vivo tissue recognition (305) and to utilize breast stromal molecular information to guide more precise BCS. 
Chapter 5

\section{Real-time lipid patterns to classify viable and necrotic liver tumours}




\begin{abstract}
Real-time tissue classifiers based on molecular patterns are emerging tools for fast tumour diagnosis. Here, rapid evaporative ionization mass spectrometry (REIMS) and multivariate statistical analysis (principal component analysis-linear discriminant analysis) were used to classify tissues with subsequent comparison to gold standard histopathology. This study explored whether REIMS lipid patterns can identify human liver tumours and improve the rapid characterization of their underlying metabolic features. REIMS based classification of liver parenchyma (LP), hepatocellular carcinoma (HCC) and metastatic adenocarcinoma (MAC) reached an accuracy of $98.3 \%$ using a leave-one-patient-out cross-validation. Lipid patterns of LP were more similar to those of HCC than to those of MAC and allowed clear distinction between primary and metastatic liver tumours. HCC lipid patterns were more heterogeneous than those of MAC, which is consistent with the variation seen in the histopathological phenotype. A common ceramide pattern discriminated necrotic from viable tumour in MAC with $92.9 \%$ accuracy and in other human tumours. Targeted analysis of ceramides and related sphingolipids mass features in necrotic tissues may provide a new classification of tumour cell death based on metabolic shifts. Real-time lipid patterns may have a role in future clinical decision-making in cancer precision medicine.
\end{abstract}

\title{
Introduction
}

The inherent heterogeneous nature of cancer renders individual patient's response to clinical interventions often unpredictable (327). Cancer precision medicine aims to improve treatment outcome through precise tumour characterization and tailoring clinical interventions to individual patient's needs (5). This may be achieved by studying the biological complexity related to tumour heterogeneity at multi-molecular levels including genomics (328), proteomics (329) and metabolomics (330). Patients may benefit from a real-time, cost-effective, and implementable technology for high-throughput tumour characterization. Such a technology could complement the quest for biological understanding and multi-omics integration in precision medicine, $(5,331,332)$, which would lead to a more straightforward clinical implementation.

As a subclass of metabolites, lipids are known to be directly involved in defining the tumour phenotype (333). Their bioactive roles have been studied in detail over the past decades, including in cancer research $(334,335)$. Lipid metabolism reprogramming has been recognized as one of the cancer hallmarks and as a potential therapeutic target (336). However, cancer precision medicine has not yet considered the full diagnostic potential of lipid metabolism $(4,6,337)$. The lipid metabolism underlying metastasis and resistance to cell death may predict disease progression and response to therapy, critical factors for informed clinical decisions.

With the development of real-time intraoperative tools to assist in situ surgical diagnosis, lipid patterns have recently gained interest as tumour-specific metabolic phenotypes (338). These new tools are able to distinguish tissue constituents based on their physicochemical properties creating tissue-specific lipid patterns for intraoperative tissue classification (2931). These real-time classifiers provide intraoperative information about the disease 
without elaborate processing and expertise such as required for histopathology, the gold standard for tumour diagnostics. They may on one hand reduce the need for perioperative tissue diagnostics by frozen tissue section analysis, and on the other hand may provide additional clinically relevant information which cannot be obtained from a haematoxylin and eosin stained frozen tissue section. Mass spectrometry (MS) is one of the analytical approaches that recently emerged as a tool for intraoperative tissue typing $(29-31,338)$. In particular, the combination of surgical instruments such as diathermia or laser scalpels with rapid evaporative ionization mass spectrometry (REIMS) has shown potential to assist surgical tumour resection and in situ tumour diagnostics (300-302,339) as REIMS technology can analyse tissue aerosols and establish mass spectrometric patterns within seconds (298). The thermal disintegration of the tissue produces singly charged molecular ions, the majority of which are lipids. Once ionized, lipids are separated based on their mass-tocharge $(\mathrm{m} / \mathrm{z})$ ratio and their relative abundance is measured. This allows the detection of tissue-specific lipid patterns (285). So far, real-time tissue classifiers based on lipid patterns have been mainly applied to intraoperative diagnostics. However, lipid patterns generated from tumour tissues may contain information relevant for clinical decisions beyond tissue diagnosis in the operating room.

The lipid patterns may provide insight into the origin of the tumour metastasis. Metastases are the most common cause of cancer death (337) and the liver is one of the main sites of tumour metastases. The invasion-metastasis cascade is a multistep process (340). Therefore, tumour metastases in the liver are expected to share common molecular alterations, which are different from those of primary liver tumours. In the current study, The lipid patterns of normal liver parenchyma (LP), primary liver tumours (hepatocellular cancer, HCC) and colorectal and breast cancer liver metastases (MAC) were compared. It can be hypothesized that the lipid patterns of LP are more similar to that of HCC than that of MAC and that the lipid patterns of MAC are more homogenous than that of HCC.

The implication of bioactive lipids, and especially ceramides, has been extensively reported in tumour cell death mechanism and treatment response (334,341-344), this study therefore included an investigation into the change of lipid patterns between viable and necrotic tumour in the current study hypothesizing that the presence of ceramides can discriminate between viable and necrotic tumour tissue.

\section{Materials and Methods}

\section{Patients}

This study included patients who underwent surgery at Maastricht University Medical Centre (MUMC+) between November 2017 and February 2020. Patients older than 18 years were eligible if they were scheduled for partial hepatectomy for removal of a liver tumour or scheduled for removal of a soft tissue tumour. The study was approved by the Medical Ethics Committee of MUMC+ (approval number METC 16-4-168) and conducted according to the revised version of the Declaration of Helsinki. Written informed consent was obtained from each patient prior to study participation. 
In total, 56 patients were included in the study (fig. 1) and 54 patients provided liver resected tissues; 53 patients provided liver resected tissues to study the classification LP/HCC/MAC. Among them, 16 were diagnosed with HCC and 37 patients were diagnosed with MAC. Among patients with MAC, two patients were diagnosed with breast cancer and 35 were diagnosed with colorectal cancer. Five patients provided MAC resected tissues after two surgical procedures on different days. One patient operated for MAC provided tissue used to test the classifier recognition.

One patient who provided a neuroendocrine liver metastasis, and three patients who provided extra-hepatic samples were included to study the tumour necrosis. For the extrahepatic samples, the first patient was operated for HCC and provided a stomach metastasis in complement to the primary HCC liver tissue. The second patient was operated for primary and liver metastasis resection surgery and provided a primary adrenocortical carcinoma. The third patient was operated for soft tissue tumour of the hip and provided a liposarcoma.

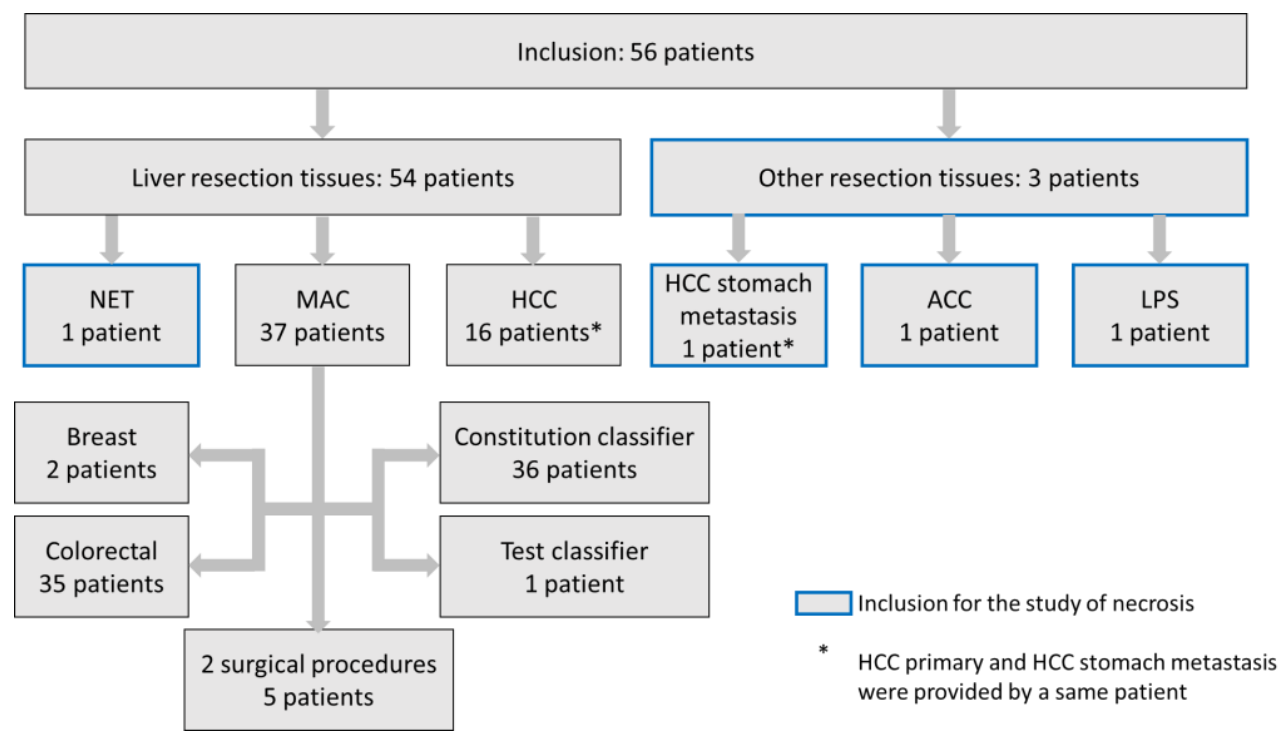

Figure 1. Diagram of the patient population. NET: neuroendocrine tumour, MAC: metastatic adenocarcinoma, HCC: hepatocellular carcinoma, ACC: adrenocortical carcinoma, LPS: liposarcoma.

\section{Tissue procurement}

The resection specimen was transferred fresh from the operating theatre to the pathology department as soon as possible. A pathologist dissected normal and tumour tissue surplus to diagnostic needs from the resection specimen for the present study. Tissue slices were used either for immediate REIMS analysis or frozen in liquid nitrogen and stored at $-80^{\circ} \mathrm{C}$ for lipid identification and precise distribution. 
REIMS analysis for tissue classification (chapter 1, fig. 6)

After collection, fresh tissue slices were placed on wiper (Kimtech, Kimberly Clark) wet with deionized water, on a silicone return electrode (Erbe). All experiments were performed in a laminar flow biosafety cabinet (Biowizard Xtra Line, Kojair Blue Series Technologies). The tissue slides were sampled using a monopolar hand-piece (Waters Research Centre, Hungary) equipped with a $1 \mathrm{~mm}$-diameter needle electrode connected to a commercially available electrosurgical heat-generator (Force FX, Covidien Ltd.). Multiple locations within the normal and tumour areas were sampled. Cut and pure modalities were used to cauterize the tissue with a power set between 8 and $25 \mathrm{~W}$ depending on the sample size and the signal quality. The generated vapours were aspirated into a REIMS interface (Waters Corporation, Wilmslow, UK) coupled to a mobile Xevo G2-XS quadrupole-time-of-flight mass spectrometer ( $Q-T o F$ MS, Waters Corporation, Wilmslow, UK). MS quality grade isopropanol (Biosolv, Honeywell) was used as a matrix (318).

The generated electrosurgical vapours were aspirated into the surgical hand-piece employing a 3 meter-long polytetrafluoroethylene tubing (PTFE), linked to a REIMS interface (Waters Corporation, Wilmslow, UK) coupled to a mobile Xevo G2-XS quadrupoletime-of-flight mass spectrometer (Q-ToF MS, Waters Corporation, Wilmslow, UK). An aspiration system (ZERO SMOG 2, Weller FT) was set-up to increase the aspiration of the vapours obtained by the venturi tube in the mass spectrometer and so to avoid the spread of electrosurgical vapours in the laboratory. A MS quality grade isopropanol (Biosolv, Honeywell) solvent used as a matrix was infused at $150 \mu \mathrm{L} / \mathrm{min}$, containing leucineencephalin (LeuEnk, Sigma-Aldrich) in solution for lock-mass correction. The concentration of LeuEnk ranged from 0.1 to $1 \mathrm{ng} / \mu \mathrm{l}$ and was adjusted on the intensity of the infused [LeuEnk-H] mass peak before tissue analysis. The tissue vapours and the solvent solution were mixed together inner a stainless steel capillary towards a heated coil. The electrosurgical vapours were analysed by REIMS in negative ionization mode and sensitivity mode. Acquisitions were performed over the mass range $\mathrm{m} / \mathrm{z} 100$ to 1500 . Instrument calibration was performed or checked on sodium formate before tissue analysis. Scan time was set to 1 second. The mass resolution for [LeuEnk-H] ${ }^{-}$was around 50000.

\section{REIMS analysis for lipid identification}

Lipid identification was based on mass accuracy and MS/MS fragmentation. A calibration or a check of the calibration of the instrument REIMS Q-TOF was performed before tissue analysis on the mass range $\mathrm{m} / \mathrm{z} 100$ to 1500 on sodium formate in negative ionization mode. REIMS tissue patterns were corrected on lock-mass deprotonated leucine-encephalin [LeuEnk-H] $]^{-}$at $\mathrm{m} / \mathrm{z} 554.2615$ and the quality of the calibration was checked on the deprotonated leucine-encephalin dimer [2LeuEnk-H] ${ }^{-}$at $\mathrm{m} / z 1109.5302$ of the background signal between the tissue samplings. 
Tandem mass spectrometry experiments were performed on Xevo G2-XS QToF (Waters Corporation, Wilmslow, UK) by collision-induced dissociation with argon gas. Experimental exact masses using external lock-mass correction and fragmentation patterns were compared to online database ALEX ${ }^{123}$ lipid calculator (319) and reference literature (345347). In addition, for chlorine adducts isotope pattern distributions were checked and compatible with the presence of chlorine in the formula.

\section{Histopathological review}

After REIMS analysis, the remaining tissue was fixed in formalin (Unifix, Klinipath) and embedded in paraffin using a standard protocol. Tissue blocks were sectioned at $5 \mu \mathrm{m}$ thickness on a microtome (Leica). Haematoxylin and eosin stained sections were prepared for histopathology review. During the review, the tissue surrounding the tissue defect from the REIMS procedure was analysed to predict the type of the evaporated tissue. Representative histology pictures for figures were taken on a microscope by a histopathologist.

\section{Data analysis}

REIMS raw data were imported in the "abstract model builder" software AMX version 0.9.2092.0 (Waters Research Centre, Budapest, Hungary). REIMS tissue scans were selected based on their intensities to create a single profile for each sampling spot (one spectrum per sampling spot). For each model, advanced 0.1 binning was selected. Model preprocessing included lock-mass correction, background subtraction and normalization.

The lipid patterns were subjected to multivariate statistical analysis: principal component analysis (PCA) and a linear discriminant analysis (LDA). The classification based on the PCALDA analysis of REIMS patterns was compared to the histopathology tissue type prediction to calculate the accuracy of the REIMS based classifier. Cross-validation was performed following a leave-one-patient-out classification method.

Data matrix from MAC viable tumour vs. MAC necrosis model were extracted from $A M X$ in Excel and individual mass feature intensities were exported into MetaboAnalyst (348) for statistical analysis as unpaired samples and peak intensity table. No missing values were detected. No data filtering, no sample normalization, no data transformation and no data scaling were performed. Unequal variance and non-parametric test were selected for univariate analysis by T-test ( $p$-value) and box plot to confirm the significant intensity differences between two tissue conditions.

Desorption electrospray ionization - mass spectrometry imaging (DESI-MSI) analysis for lipid identification and for lipid in situ distribution

Tissue block of one tumour sample was taken out of the low temperature freezer, sectioned on a cryotome (Microm) at $8 \mu \mathrm{m}$-thickness. Section was thaw mounted on a regular 
histologic slide (Superfrost, VWR) and stored in a low temperature freezer until further usage. Before DESI-MSI, tissue section was dried in a desiccator for $20 \mathrm{~min}$. DESI-MSI experiment was performed on a DESI source coupled to a Xevo G2-XS QToF (Waters Corporation, Wilmslow, UK). Solvent was methanol:water (Biosolv) in ratio 98:2. Inlet capillary was heated at 500 degrees Celsius approximately. Acquisition was performed in negative ionization mode, sensitivity mode, over the mass range m/z 50 to 1800 at $50 \times 50$ $\mu \mathrm{m}^{2}$ pixel size, with a flowrate of $2 \mu \mathrm{l} / \mathrm{min}$ for one scan per second. After experiment, the tissue section was stained by haematoxylin \& eosin, scanned under a Mirax Desk digital slide scanner (Zeiss) and annotated by a liver pathologist.

a)

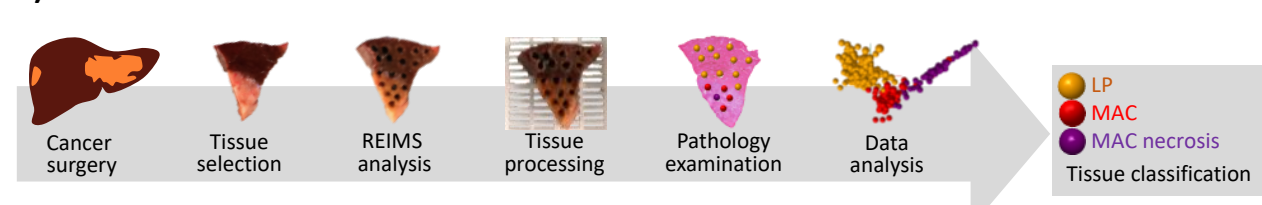

b)

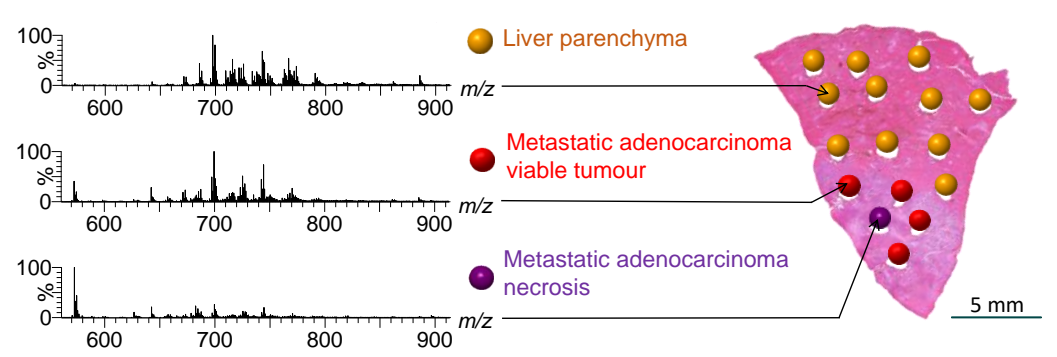

Figure 2. Workflow. (a) General workflow. (b) Pathology examination of the REIMS tissue samplings. Each lipid pattern generated by REIMS was attributed to a tissue component based on the histopathology of the remaining tissue surrounding each sampling spot.

\section{DESI-MS analysis for lipid identification}

For identification based on mass accuracy, a calibration of the instrument REIMS Q-TOF was performed before tissue analysis on sodium formate in negative ionization mode. REIMS spectra were corrected on lock-mass deprotonated raffinose $\mathrm{m} / \mathrm{z} 503.1606[\mathrm{M}-\mathrm{H}]^{-}$. Experimental exact masses were tested on ALEX ${ }^{123}$ lipid calculator (319). Tandem mass spectrometry experiments were performed on Xevo G2-XS QToF (Waters Corporation, Wilmslow, UK) by collision-induced dissociation with argon gas. Fragmentation patterns were compared to reference literature $(346,347)$.

\section{Results}

To study the two hypotheses, ex vivo REIMS analysis of electrosurgical vapours was used to establish the lipid patterns from multiple different locations of fresh resected tissues of 56 
patients who had surgery for tumour resection (fig. 1), including 16 primary liver cancer (table 1) and 36 liver metastases (table 3). In total, 2424 lipid patterns were generated with subsequent histopathological tissue review to build a library of tissue-specific lipid patterns.

Table 1. Number of REIMS patterns generated per tissue type and per patient in the analysis of hepatocellular carcinoma (HCC) resected tissues.

\begin{tabular}{|c|c|c|c|c|c|}
\hline Patient & Total & LP & HCC mix & HCC vt & HCC nec \\
\hline HCC 01 & 33 & & 33 & 33 & \\
\hline HCC 02 & 79 & & 79 & 79 & \\
\hline HCC 03 & 39 & 14 & 25 & 25 & \\
\hline HCC 05 & 83 & 20 & 63 & 63 & \\
\hline HCC 06 & 88 & 19 & 69 & 58 & 4 \\
\hline HCC 07 & 65 & 48 & 17 & 15 & \\
\hline HCC 08 & 56 & 24 & 32 & 32 & \\
\hline HCC 09 & 73 & 24 & 49 & 47 & 2 \\
\hline HCC 10 & 17 & 12 & 5 & 5 & \\
\hline HCC 11 & 41 & & 41 & 28 & 6 \\
\hline HCC 12 & 135 & 31 & 104 & 99 & \\
\hline HCC 13 & 60 & 49 & 11 & 11 & \\
\hline HCC 14 & 31 & & 31 & 13 & 6 \\
\hline HCC 15 & 36 & 20 & 16 & & 1 \\
\hline HCC 16 & 18 & & 18 & 18 & \\
\hline All & 885 & 284 & 601 & 534 & 19 \\
\hline
\end{tabular}

Table 2. Number of REIMS patterns generated per tissue type and per patient in the analysis of non-MAC non-HCC liver resected tissues.

\begin{tabular}{|c|c|c|c|}
\hline Patient & Total & vt & nec \\
\hline ACC 01 & 23 & 9 & 14 \\
\hline LPS 01 & 9 & 8 & 1 \\
\hline NET 01 & 41 & 18 & 23 \\
\hline S-HCC 01 & 21 & 5 & 16 \\
\hline
\end{tabular}

\author{
HCC: hepatocellular carcinoma \\ MAC: metastatic adenocarcinoma \\ LP: liver parenchyma \\ ACC: adrenocortical carcinoma \\ LPS: liposarcoma \\ NET: neuroendocrine tumour \\ $\mathrm{S}-\mathrm{HCC}$ : stomach HCC metastasis \\ vt: viable tumour \\ nec: necrosis \\ mix: viable, necrosis, fibrosis, etc.
}

Lipid patterns were subjected to multivariate statistical analysis employing a combination of both principal component analysis and linear discriminant analysis (PCA-LDA) as follow:

(1) Unsupervised principal component analysis (PCA) was used for data reduction and to provide an overview of the variance of the lipid patterns included in the model; PCA analysis was performed on a given mass range or on specified mass features of the lipid patterns (as detailed for each analysis);

(2) Supervised linear discriminant analysis (LDA) was used to further optimize the separation between different tissue types by means of projected classes and to reduce the variance within each projected class used for pattern recognition (and therefore for tissue classification).

The tissue classification predicted by PCA-LDA based on the REIMS lipid patterns was compared to the histopathological classification to calculate the accuracy of the lipid patterns based classifier. Cross-validation was performed following a leave-one-patient-out method. Confusion matrices were generated to assess the accuracy of the tissue classification. PCA score plots provided an overview of the variance of the lipid patterns. Notably, as unsupervised analysis method, the PCA score plots did not consider the histopathological classification, while the LDA adds that information to the classifier. 


\section{Lipid patterns classify liver parenchyma and liver tumours}

To test the first hypothesis, the classification of liver parenchyma (LP), primary liver tumour (i.e. hepatocellular carcinoma (HCC)), and colorectal and breast cancer liver metastases (i.e. metastatic adenocarcinoma (MAC)) was employed. The overall accuracy of the classification of HCC and LP predicted by the PCA-LDA analysis of REIMS lipid patterns compared to the histopathological classification was very good (96.8\%) but slightly lower than the overall accuracy of classification of MAC and LP (99.4\%) (fig. 3a-b). The classification of LP, HCC and MAC reached $98.3 \%$ accuracy (fig. 3c). The classification between HCC and MAC reached $97.4 \%$ accuracy (fig. 3d). These results demonstrate the ability of the lipid pattern library to predict the tissue class with good accuracy. In addition, these results indicate PCA-LDA based analysis of lipid patterns appears to be better in recognizing MAC than HCC. Representative lipid patterns and pseudo-LDA score plots are shown in figure 4.

Table 3. Number of REIMS patterns generated per tissue type and per patient in the analysis of metastatic adenocarcinoma (MAC) resected tissues. LP: liver parenchyma, nec: necrosis, vt: viable tumour, mix: viable tumour, necrosis, fibrosis, etc.

\begin{tabular}{|c|c|c|c|c|c|}
\hline \multirow{2}{*}{ Patient } & \multirow{2}{*}{ Total } & \multirow{2}{*}{ LP } & \multicolumn{3}{|c|}{ MAC } \\
\hline & & & $\operatorname{mix}$ & vt & nec \\
\hline MAC 01 & 9 & & 9 & & \\
\hline MAC 02 & 22 & 2 & 20 & & \\
\hline MAC 03 & 15 & 9 & 6 & & 6 \\
\hline MAC 04 & 32 & 14 & 18 & & 18 \\
\hline MAC 05 & 14 & 9 & 5 & 3 & \\
\hline MAC 06 & 24 & 14 & 10 & & 9 \\
\hline MAC 07 & 22 & 9 & 13 & 13 & \\
\hline MAC 08 & 80 & 33 & 47 & & \\
\hline MAC 09 & 74 & 58 & 16 & 12 & 1 \\
\hline MAC 10 & 36 & 15 & 21 & 8 & 2 \\
\hline MAC $11^{*}$ & 13 & 6 & 7 & 7 & \\
\hline MAC 12 & 49 & 32 & 17 & 12 & \\
\hline MAC 13 & 44 & 23 & 21 & & 17 \\
\hline MAC 14 & 21 & 15 & 6 & 1 & \\
\hline MAC 15 & 26 & 22 & 4 & 3 & \\
\hline MAC 16 & 106 & 51 & 55 & 12 & 4 \\
\hline MAC 17 & 23 & 14 & 9 & & 5 \\
\hline MAC 18 & 18 & 13 & 5 & 2 & \\
\hline
\end{tabular}

\begin{tabular}{|c|c|c|c|c|c|}
\hline \multirow[t]{2}{*}{ Patient } & \multirow[t]{2}{*}{ Total } & \multirow[t]{2}{*}{ LP } & \multicolumn{3}{|c|}{ MAC } \\
\hline & & & $\operatorname{mix}$ & vt & nec \\
\hline MAC 19 & 26 & 23 & 3 & & \\
\hline MAC 20 & 104 & 38 & 66 & & 4 \\
\hline MAC 21 & 54 & 24 & 30 & 2 & 6 \\
\hline MAC 22 & 14 & 14 & & & \\
\hline MAC 23 & 5 & 5 & & & \\
\hline MAC 24 & 16 & 10 & 6 & 1 & \\
\hline MAC 25 & 11 & 8 & 3 & 3 & \\
\hline MAC 26 & 27 & 14 & 13 & 2 & \\
\hline MAC 27 & 17 & 16 & 1 & & 1 \\
\hline MAC 28 & 28 & 16 & 12 & & \\
\hline MAC 29 & 115 & 75 & 40 & 16 & 4 \\
\hline MAC 30 & 90 & 74 & 16 & 6 & \\
\hline MAC 31 & 78 & 41 & 37 & 1 & \\
\hline MAC 32 & 23 & & 23 & & 1 \\
\hline MAC 33 & 35 & 27 & 8 & & 1 \\
\hline MAC 34 & 91 & 35 & 56 & 2 & \\
\hline MAC 35 & 68 & 40 & 28 & 5 & \\
\hline MAC 36* & 15 & 9 & 6 & 6 & \\
\hline All & 1445 & 808 & 637 & 117 & 79 \\
\hline
\end{tabular}

Next, the PCA score plots were used to evaluate the variance of the lipid patterns used for the predicted classification before supervised statistical analysis by LDA. On the PCA score plots with only 2 tissue classes, HCC and LP lipid patterns were relatively close to each other (fig. 5a) whereas MAC and LP were relatively well separated (fig. 5b). Figure 5c supports these observations showing that LP, HCC and MAC lipid patterns were ordered and aligned along the PC1 axis (PC1 loading plot suggested identification of some mass features in fig. 6 
and table 4). Based on these visual assessments, lipid patterns are more similar comparing LP and HCC than between LP and MAC.

Representative histopathological pictures of the 3 tissue types used to define the actual class were included (fig. $\mathbf{5 d - f}$ ). While LP and HCC are both mainly composed of hepatocytes and hepatocyte derived malignant cells, MAC is composed of malignant epithelial cells originating from the colorectal and the breast primary tumours. The histopathological phenotype of HCC was more similar to that of LP than that of MAC supporting the lipid pattern findings.

a)

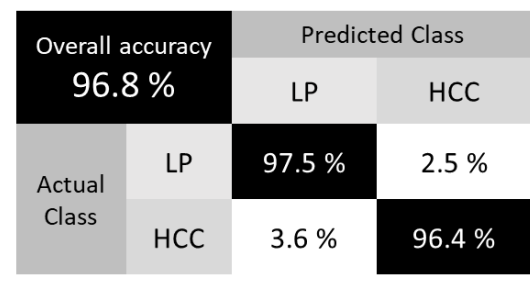

c)

\begin{tabular}{c|c|c|c|c}
\hline $\begin{array}{c}\text { Overall accuracy } \\
98.3 \%\end{array}$ & \multicolumn{3}{|c}{ Predicted Class } \\
\hline & LP & $98.8 \%$ & $0.3 \%$ & $0.2 \%$ \\
\hline $\begin{array}{c}\text { Actual } \\
\text { Class }\end{array}$ & HCC & $4.3 \%$ & $93.4 \%$ & $0.2 \%$ \\
& MAC & $0.9 \%$ & $0.0 \%$ & $\mathbf{8 9 . 7} \%$ \\
\hline
\end{tabular}

b)

\begin{tabular}{|c|c|c|c|}
\hline \multirow{2}{*}{\multicolumn{2}{|c|}{$\begin{array}{c}\text { Overall accuracy } \\
99.4 \%\end{array}$}} & \multicolumn{2}{|c|}{ Predicted Class } \\
\hline & & LP & MAC \\
\hline \multirow{2}{*}{$\begin{array}{c}\text { Actual } \\
\text { Class }\end{array}$} & LP & $99.4 \%$ & $0.6 \%$ \\
\hline & MAC & $0.9 \%$ & $99.1 \%$ \\
\hline
\end{tabular}

d)

\begin{tabular}{|c|c|c|c|}
\hline \multirow{2}{*}{\multicolumn{2}{|c|}{$\begin{array}{c}\text { Overall accuracy } \\
97.4 \%\end{array}$}} & \multicolumn{2}{|c|}{ Predicted Class } \\
\hline & & $\mathrm{HCC}$ & MAC \\
\hline \multirow{2}{*}{$\begin{array}{c}\text { Actual } \\
\text { Class }\end{array}$} & $\mathrm{HCC}$ & $97.8 \%$ & $2.5 \%$ \\
\hline & MAC & $4.3 \%$ & $95.7 \%$ \\
\hline
\end{tabular}

Figure 3. Confusion matrices for the classification of liver parenchyma (LP), hepatocellular carcinoma (HCC) and metastatic adenocarcinoma (MAC) by lipid patterns. The tissue classification provided by the histopathologist was used as actual class.

(a) Classification of 818 lipid patterns (284 LP, 534 HCC) generated from 16 patients using 30 principal component (PC) dimensions, a mass range $\mathrm{m} / \mathrm{z} 600-900$ resulted in no outlier. The overall accuracy by lipid pattern was $96.8 \%$, ranging from $96.4 \%$ to $97.5 \%$ and was highest for LP.

(b) Classification of 925 lipid patterns ( 808 LP and 117 MAC) generated from 34 patients using 5 PC dimensions, a mass range $m / z 600-900$ and resulting in 0 outlier. The overall accuracy by lipid pattern was $99.4 \%$, ranging from $99.1 \%$ to $99.4 \%$ and was highest for LP.

(c) Classification of 1743 lipid patterns (1092 LP, 534 HCC, 117 MAC) generated from 50 patients using 55 PC dimensions, a mass range $\mathrm{m} / \mathrm{z} 600-900$ resulted in 30 outliers. The overall accuracy by lipid pattern was $98.3 \%$, ranging from $89.7 \%$ to $98.8 \%$ and was highest for LP.

(d) Classification of 651 lipid patterns ( 534 HCC, $117 \mathrm{MAC}$ ) generated from 35 patients using 15 PC dimensions, a mass range $\mathrm{m} / \mathrm{z} 600-900$ and resulting in 0 outlier. The overall accuracy by lipid pattern was $97.4 \%$, ranging from $95.7 \%$ to $97.8 \%$ and was highest for HCC. 
a)

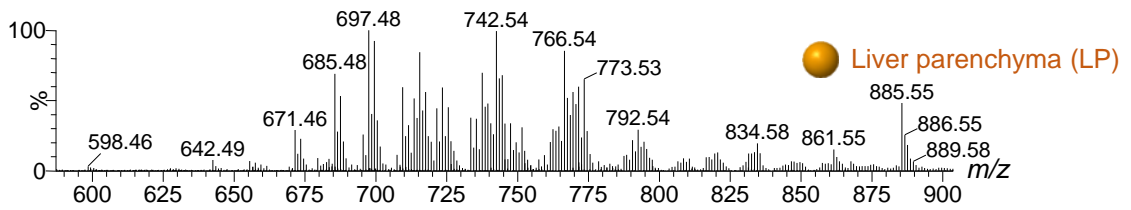

b)

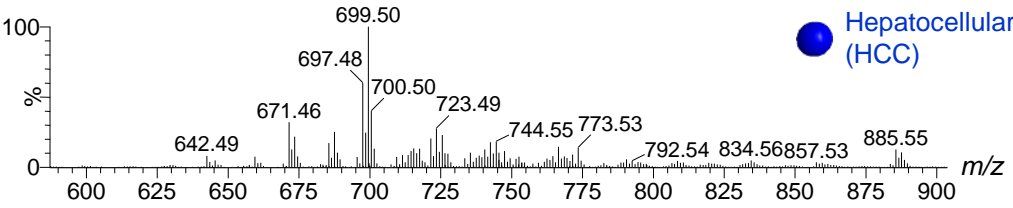

c)

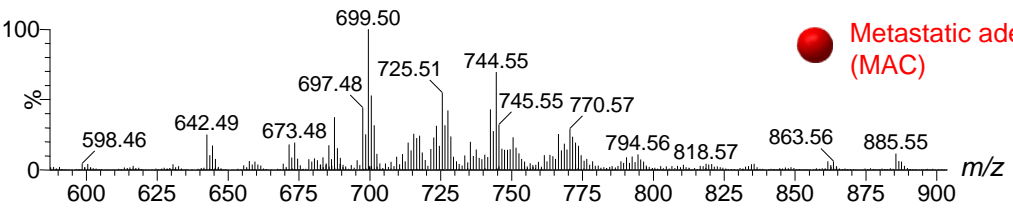

d)

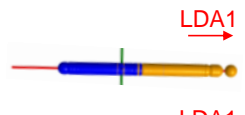

e)

f)

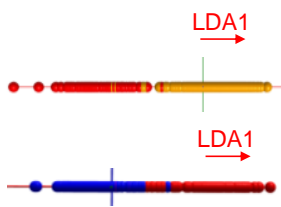

g)

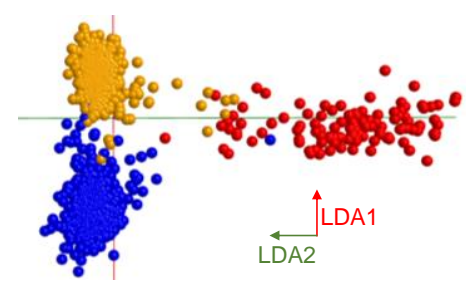

Figure 4. REIMS lipid patterns and pseudo-LDA score plots for the classification of LP and liver tumours.

(a) Single REIMS spectrum for liver parenchyma (LP).

(b) Single REIMS spectrum for hepatocellular carcinoma (HCC).

(c) Single REIMS spectrum for metastatic adenocarcinoma (MAC).

(d) Linear discriminant analysis (LDA) score plot for the classifications of HCC and LP.

(e) LDA score plot for the classifications of MAC and LP.

(f) LDA score plot for the classifications of HCC and MAC.

(g) Pseudo-LDA score plot for the classifications of LP, HCC and MAC. 
a)

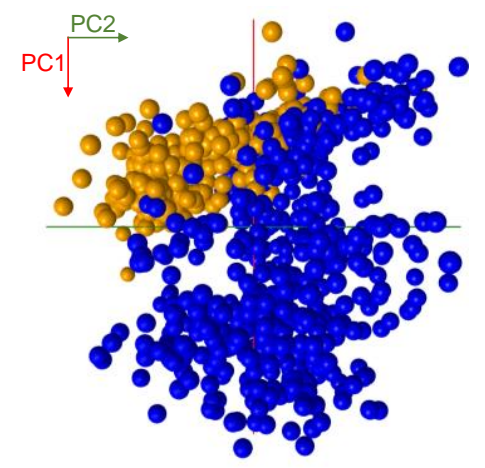

c)

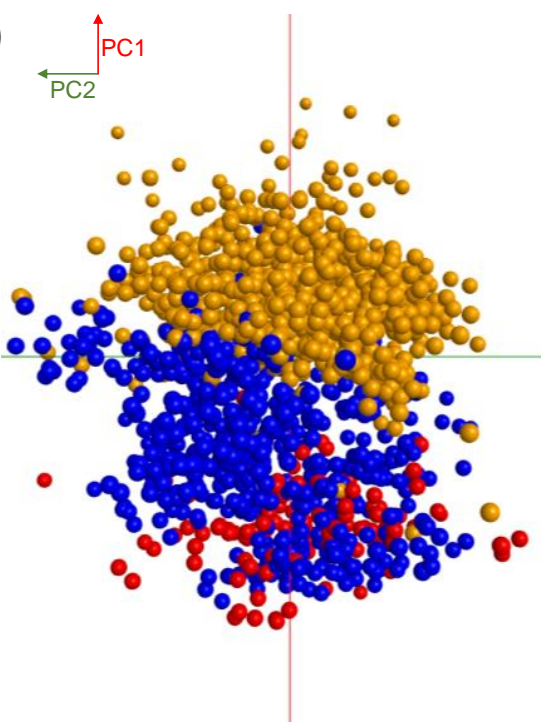

b)

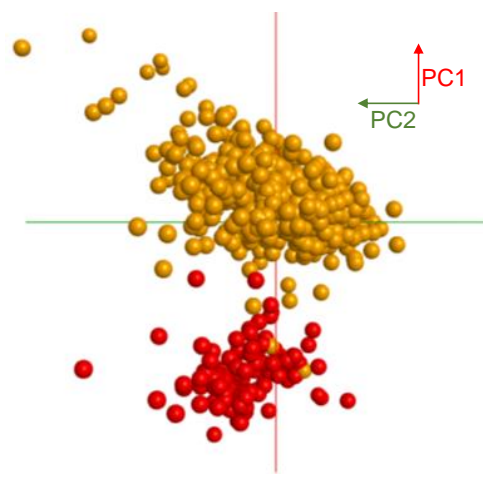

d)

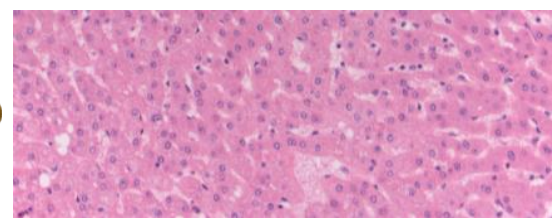

e)

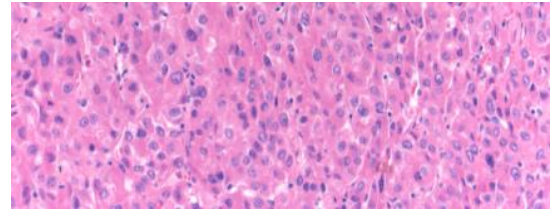

f)

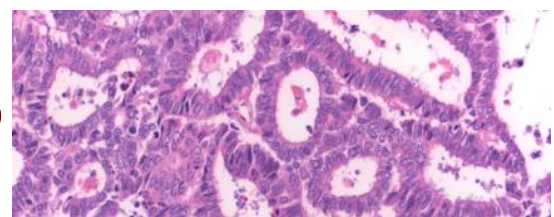

$\underbrace{\text { Liver parenchyma }}_{(\mathrm{LP})}$ Hepatocellular carcinoma
viable tumor (HCC)
Metastatic adenocarcinoma
viable tumor (MAC)

Figure 5. Principal component analysis score plots of the lipid patterns of liver parenchyma (LP), hepatocellular carcinoma (HCC) and metastatic adenocarcinoma (MAC) and representative histology.

(a) Principal component analysis (PCA) score plot (PC1 24.4\%, PC2 16.9\%) of 818 patterns (284 LP and 534 HCC) generated on tissues from 16 patients to differentiate HCC from LP.

(b) PCA score plot (PC1 30.0\%, PC2 16.3\%) of 995 patterns (878 LP and 117 MAC) generated on tissues from 36 patients to differentiate MAC from LP.

(c) PCA score plot (PC1 describes $25.4 \%$ of the total variance of the data, PC2 14.9\%) of 1813 patterns (1162 LP, $534 \mathrm{HCC}, 117 \mathrm{MAC}$ ) generated on tissues from 52 patients.

(d) Representative histology (haematoxylin \& eosin (H\&E) staining, original magnification x200) for LP.

(e) Representative histology (haematoxylin \& eosin (H\&E) staining, original magnification x200) for HCC.

(f) Representative histology (haematoxylin \& eosin (H\&E) staining, original magnification x200) for MAC. 


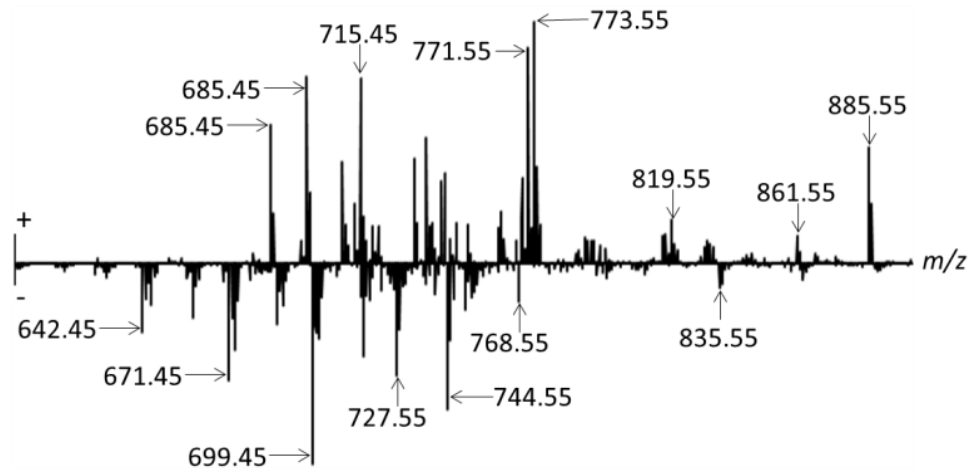

Figure 6. Principal component 1 (PC1) mass features loading plot of the LP/HCC/MAC PCA model of figure 5c.

Table 4. Lipid identification by REIMS based on mass accuracy and tandem mass spectrometry for the first hypothesis (primary and metastatic liver tumours).

\begin{tabular}{|c|c|c|c|c|c|c|c|}
\hline Identification & Adduct & Formula & $\begin{array}{l}\text { PCA } \\
m / z\end{array}$ & $\begin{array}{c}\text { Measured } \\
\mathrm{m} / \mathrm{z}\end{array}$ & $\begin{array}{c}\text { Theoretical } \\
m / z\end{array}$ & $\begin{array}{c}\text { Mass error } \\
\text { (ppm) }\end{array}$ & $\begin{array}{l}\text { Fragment ions } \\
\text { upon MS/MS }\end{array}$ \\
\hline $\begin{array}{l}\text { PA 36:2 } \\
\text { / PE 34:1* }\end{array}$ & $\begin{array}{c}{[\mathrm{M}-\mathrm{H}]^{-}} \\
/\left[\mathrm{M}-\mathrm{NH}_{3}\right]^{-}\end{array}$ & $\mathrm{C}_{39} \mathrm{H}_{72} \mathrm{O}_{8} \mathrm{P}$ & 699.45 & 699.50 & 699.50 & 2.0 & $\begin{array}{c}435.2,417.2,281.2 \\
153.0,79.0\end{array}$ \\
\hline PE 36:1 & {$[\mathrm{M}-\mathrm{H}]^{-}$} & $\mathrm{C}_{41} \mathrm{H}_{79} \mathrm{NO}_{8} \mathrm{P}$ & 744.55 & 744.56 & 744.55 & 1.9 & $\begin{array}{c}480.3,462.3,281.2 \\
153.0,79.0\end{array}$ \\
\hline PG 36:2 & {$[\mathrm{M}-\mathrm{H}]^{-}$} & $\mathrm{C}_{42} \mathrm{H}_{78} \mathrm{O}_{10} \mathrm{P}$ & 773.55 & 773.53 & 773.53 & 4.1 & $\begin{array}{c}\text { 699.5, 509.3, 491.3, } \\
435.3,417.2,281.2, \\
153.0,79.0\end{array}$ \\
\hline
\end{tabular}

\section{Lipid patterns reflect tumour heterogeneity}

The variation of the HCC patterns to the MAC patterns was further compared as a surrogate of tumour heterogeneity. The visual assessment of the PCA score plot of HCC and MAC showed that the distribution of the HCC lipid patterns is more widespread than the MAC lipid patterns (fig. 7a) suggesting that lipid patterns within HCC are more heterogeneous compared to MAC. 8 representative tumours were selected to visualize patient-specific tumour lipid patterns (fig. 7b). The inter-patient variation in lipid patterns was much smaller for MAC compared to HCC (fig. 7b) suggesting that HCC lipid patterns vary substantially between different patients, whereas the lipid patterns of colorectal cancer metastasis from different patients are very similar. PCA score plots with the lipid patterns for MAC and HCC for all the patients can be found in figure $\mathbf{8}$.

Furthermore, the histopathological phenotypes of the $4 \mathrm{HCC}$ (fig. 7c) and the 4 MAC (fig. 7d) were compared in figure 7b. While HCC histology ranged from well-differentiated to poorly differentiated including macrotrabecular, glandular-like and steatohepatitic variants (fig. 7c), all MAC showed a growth pattern typical for well-differentiated adenocarcinoma (fig. 7d), suggesting a link between the diversity of lipid patterns and histological phenotypes. 
a)

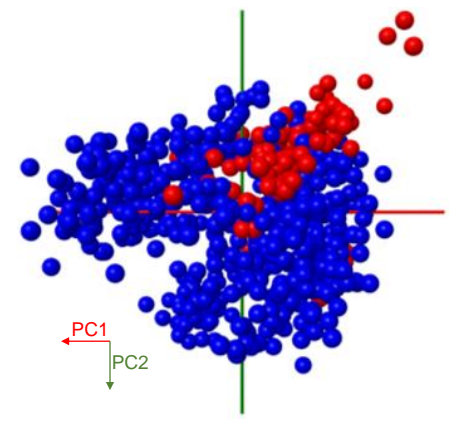

Hepatocellular carcinoma viable tumor ( $\mathrm{HCC})$

Metastatic adenocarcinoma
viable tumor (MAC)

c)

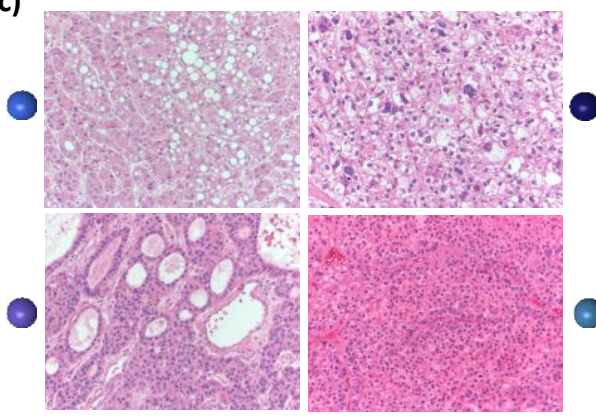

b)

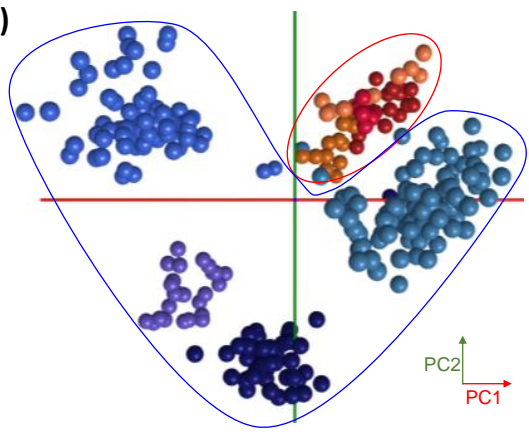

HCC viable tumor for 4 patients 1 colour for patterns of 1 patient MAC viable tumor for 4 patients 1 colour for patterns of 1 patient d)

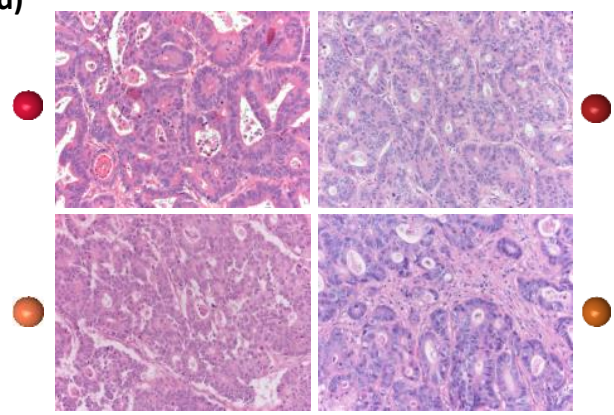

Figure 7. Tumour type-specific patterns and patient tumour-specific patterns for hepatocellular carcinoma (HCC) and metastatic adenocarcinoma (MAC).

(a) Tumour type-specific principal component analysis (PCA) score plot (PC1 23.7\%, PC2 15.0 \%, mass range $\mathrm{m} / \mathrm{z}$ 600-900) of 635 lipid patterns ( 534 HCC, $117 \mathrm{MAC}$ ) generated on tissues from 35 patients.

(b) Patient-specific PCA score plot (PC1 35.4\%, PC2 $22.3 \%$, mass range $\mathrm{m} / \mathrm{z} 600-900$ ) of 303 lipid patterns (250 HCC and $53 \mathrm{MAC}, 4$ patients per tumour type, 8 patients in total). Each colour represents the lipid patterns generated from the tissues of one patient.

(c) Histopathology for each patient HCC tumour shown in (B) (H\&E staining, original magnification x200).

(d) Histopathology for each patient MAC tumour shown in (B) (H\&E staining, original magnification x200).

a)

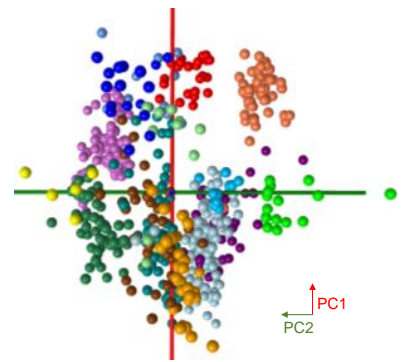

b)

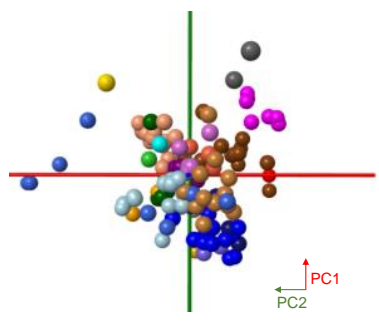

Figure 8. Patient-specific viable liver tumour patterns for hepatocellular carcinoma (HCC) and metastatic adenocarcinoma (MAC). Each colour represents the patterns generated on the viable tumour tissues of one patient.

(a) PCA score plot of $534 \mathrm{HCC}$ patterns from 15 patients (3 PC, PC1 28.5\%, PC2 18.6\%, mass range $\mathrm{m} / \mathrm{z} 700-900$ ).

(b) PCA score plot of 117 MAC patterns from 20 patients (3 PC, PC1 25.6\%, PC2 20.4\%, mass range $\mathrm{m} / \mathrm{z} 700-900$ ). 
a)

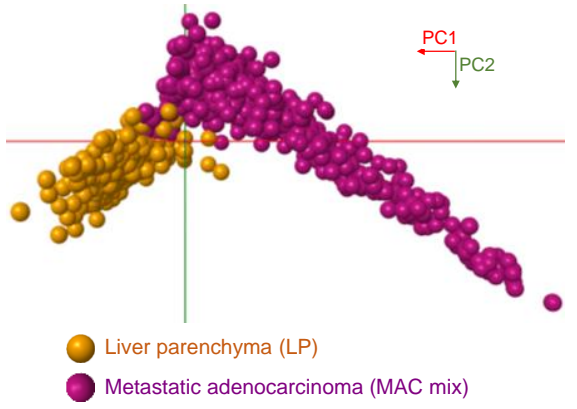

d)

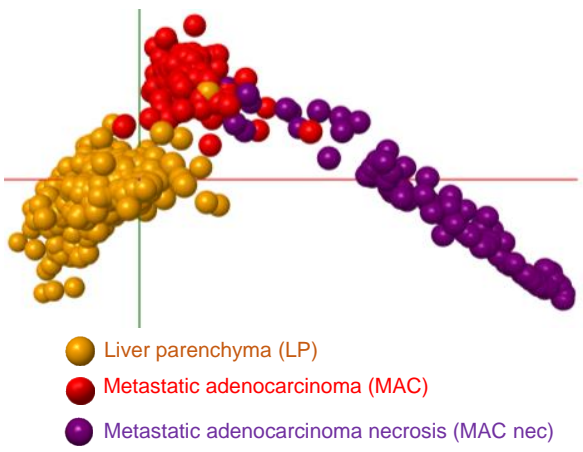

g)

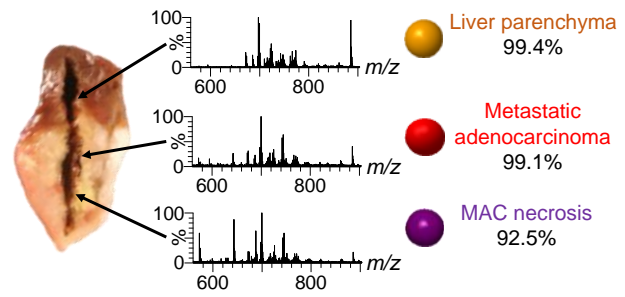

b)

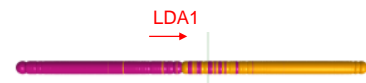

c)

\begin{tabular}{|c|c|c|c|}
\hline \multirow{2}{*}{\multicolumn{2}{|c|}{$\begin{array}{c}\text { Overall accuracy } \\
98.5 \%\end{array}$}} & \multicolumn{2}{|c|}{ Predicted Class } \\
\hline & & LP & MAC mix \\
\hline \multirow{2}{*}{$\begin{array}{l}\text { Actual } \\
\text { Class }\end{array}$} & LF & $99.1 \%$ & $0.9 \%$ \\
\hline & MAC mix & $2.0 \%$ & $98.0 \%$ \\
\hline
\end{tabular}

e)

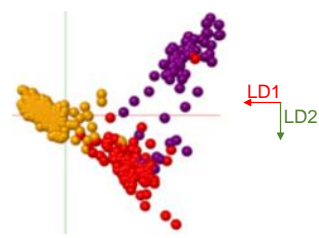

f)

\begin{tabular}{|c|c|c|c|c|}
\hline \multirow{2}{*}{\multicolumn{2}{|c|}{$\begin{array}{c}\text { Overall accuracy } \\
98.1 \%\end{array}$}} & \multicolumn{3}{|c|}{ Predicted Class } \\
\hline & & LP & MAC & MAC nec \\
\hline \multirow{3}{*}{$\begin{array}{l}\text { Actual } \\
\text { Class }\end{array}$} & LF & $99.0 \%$ & $0.5 \%$ & $0 \%$ \\
\hline & MA & $0.9 \%$ & $97.4 \%$ & $1.7 \%$ \\
\hline & & $0 \%$ & $13.9 \%$ & $86.1 \%$ \\
\hline
\end{tabular}

h)

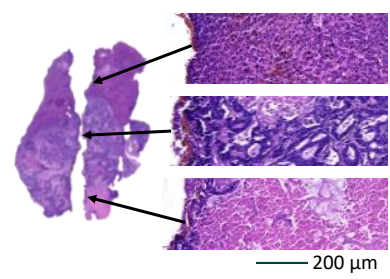

Figure 9. Recognition of tissue types in a metastatic adenocarcinoma (MAC) liver resected specimen. (a) PCA score plot for the classification of 1445 REIMS patterns including 808 liver parenchyma (LP) patterns, $637 \mathrm{MAC}$ mix (including viable tumour, necrosis, fibrosis, etc.) patterns and generated on tissues from 36 patients operated for MAC liver resection (PC1 54.8\%, PC2 14.1\%, 15 PC dimensions, mass range $m / z$ 500-1000, no outlier).

(b) Linear discriminant analysis (LDA) score plot for the classification of LP and MAC mix.

(c) Confusion matrix for the classification of LP and MAC mix.

(d) PCA score plot for the classification of 1004 REIMS patterns including 808 LP, 117 MAC and 79 MAC necrosis patterns generated from 28 patients operated for MAC liver resection (PC1 53.8\%, PC2 11.4\%, mass range $m / z$ 500-1000, 15 PC dimensions, 2 LDA dimensions, 6 outliers).

(e) Pseudo-LDA score plot for the classification of LP, MAC and MAC necrosis.

(f) Confusion matrix for the classification of LP, MAC and MAC necrosis

(g) Test of the classifier on a resected specimen of a patient operated for MAC resection: recognition of LP, MAC and MAC necrosis based on lipid patterns on the unprocessed tissue sample after surgery with guideline probability based on the class distance within the LDA space.

(h) Confirmation of the tissue types cauterized to test the classifier with pathology examination after tissue processing with haematoxylin and eosin staining. 


\section{Real-time tissue recognition of a liver metastatic adenocarcinoma specimen}

In total, 1445 patterns were included in the library of MAC resected specimens provided from 36 patients (fig. 9a-c). Classification of LP, MAC viable tumour and MAC necrosis reached $98.1 \%$ accuracy (fig. 9d-f). The classifier was tested on a specimen from patient external to the patterns library of the model (fig. $\mathbf{9 g}-\mathbf{h}$ ). The model enabled recognition of the 3 tissue components based on the lipid patterns generated in real-time during the same acquisition. After tissue processing, pathology examination confirmed the presence of LP, MAC viable tumour and MAC necrosis on the borders of the transected part of the specimen. This demonstrates the ability of lipid patterns to provide pathology feedback during surgery.

\section{Discrimination of viable and necrotic tumour parts of metastatic adenocarcinomas}

Primary HCCs but also metastases in the liver can be composed of a mixture of viable and necrotic tumour. Indeed, in colorectal cancer, necrotic debris in the tumour lumen, so called 'dirty necrosis' is characteristic for this tumour type (349). In other tumours, necrotic tumour is often interpreted as a sign of hypoxia (350). To test our second hypothesis, 196 lipid patterns obtained from the viable tumour parts to the necrotic parts in MAC of 29 patients were compared (sampling detailed in table 3 ). The lipid patterns generated via REIMS technology were very different in viable and necrotic MAC and could be distinguished with $92.9 \%$ accuracy (table 5 ).

Table 5. Confusion matrix for the classification of viable metastatic adenocarcinoma (MAC) and necrotic MAC (MAC necrosis) predicted by lipid patterns. The histopathological tissue classification was used as gold standard (actual class). Classification of 196 patterns (117 viable, 79 necrotic) generated from tissues of 29 patients using 20 PC dimensions, a mass range $\mathrm{m} / \mathrm{z} 200-1100$ and resulting in no outlier. The mean class prediction by lipid pattern was $92.9 \%$, ranging from $86.1 \%$ to $97.5 \%$ and was best for MAC viable tumour.

\begin{tabular}{|c|c|c|c|}
\hline \multirow{2}{*}{\multicolumn{2}{|c|}{$\begin{array}{c}\text { Overall class rate } \\
92.9 \%\end{array}$}} & \multicolumn{2}{|c|}{ Predicted Class } \\
\hline & & MAC & MAC \\
\hline \multirow{2}{*}{$\begin{array}{c}\text { Actual } \\
\text { Class }\end{array}$} & MAC & $97.5 \%$ & $2.6 \%$ \\
\hline & $\begin{array}{c}\text { MAC } \\
\text { necrosis }\end{array}$ & $13.9 \%$ & 86.1 \\
\hline
\end{tabular}

Next, the variance of the lipid patterns between viable and necrotic tumour parts was further investigated. The PCA score plot displayed a separation of MAC and MAC necrosis along the PC1 (explaining $65.0 \%$ of the variance) axis (fig. 10a). PC1 loading plot was used 
a)

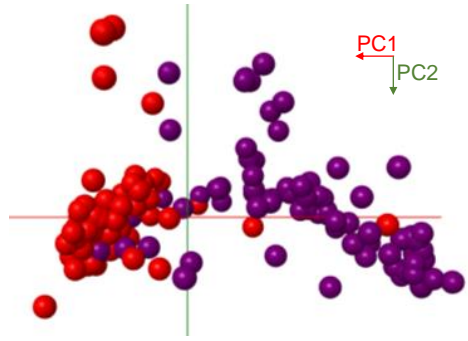

b)

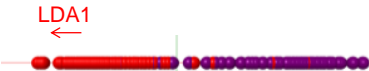

Metastatic adenocarcinoma viable tumor (MAC)

- Metastatic adenocarcinoma necrosis (MAC necrosis)

c)

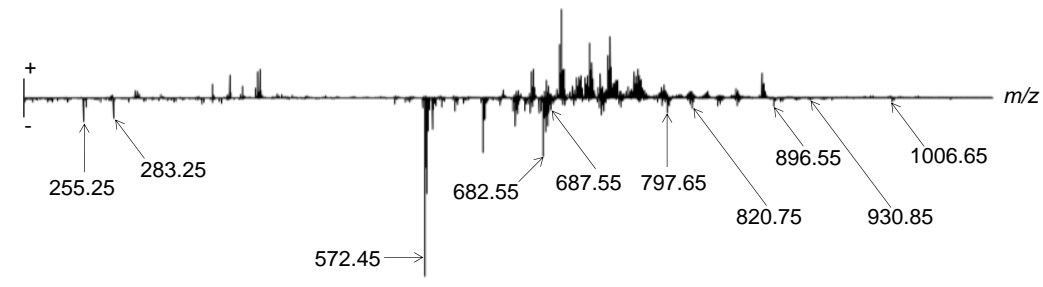

d)

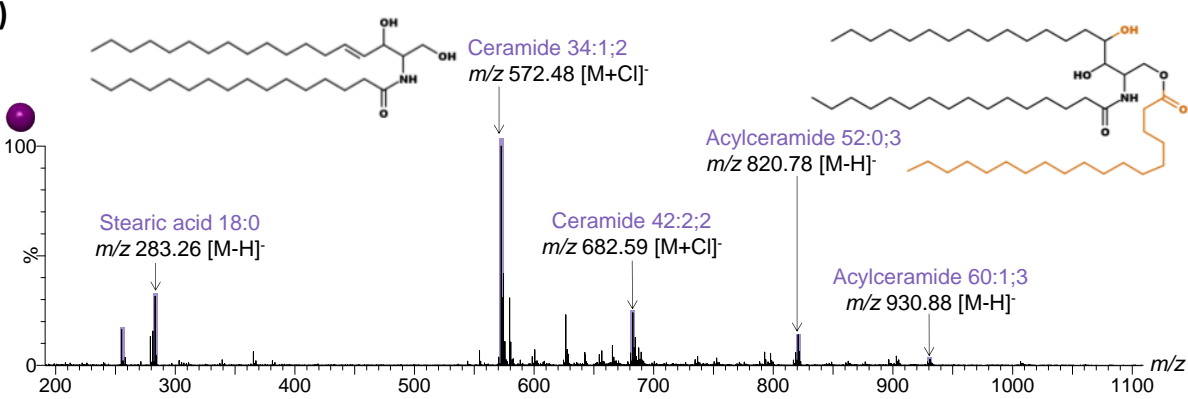

e)

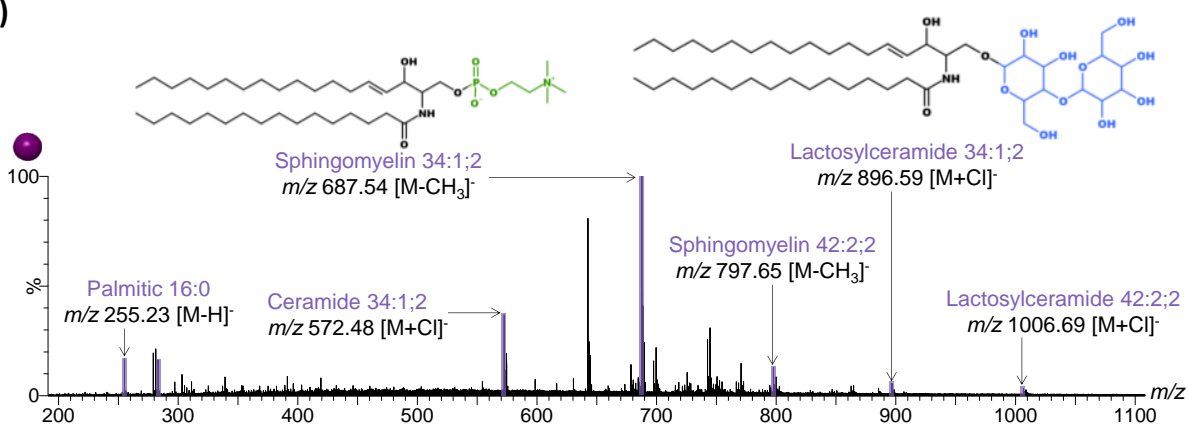

Figure 10. Discrimination between necrotic and viable metastatic adenocarcinomas.

(a) PCA score plot (PC1 65.0\%, PC2 10.3\%) for the classification of 196 metastatic adenocarcinoma (MAC) lipid patterns (117 MAC (103 colorectal, 11 breast) and 79 MAC necrosis (79 colorectal)) generated from tissues of 29 patients using 20 PC dimensions and a mass range $m / z$ 200-1100. (b) LDA score plot of 196 MAC patterns and 79 MAC necrosis. (c) PC1 loading plot indicating which mass features contributed to the PC1 dimension. Single REIMS spectrum for necrotic MAC from (d) one patient and from (e) another patient. Highlighted in violet mass spectral peaks discriminative of necrotic MAC from viable MAC based on the PC1 loading plot. Assigned representative molecular structure for mass spectral peaks $\mathrm{m} / \mathrm{z} 572.48,820.78,687.54$ and 896.59 as ceramide 34:1;2, acylceramide 52:0;3, sphingomyelin 34:1;2 and lactosylceramide 34:1;2 respectively. Representative ceramide backbone as d18:1/16/0 in black, diverse moieties in colours. 
to screen the mass features discriminative of MAC necrosis (fig. 10b). Among these mass features, fatty acids and sphingolipids including ceramides, acylceramides, sphingomyelins and lactosylceramides were identified (fig. 11, tables 6 and 7). T-tests confirmed a significant increase in MAC necrosis (fig. 12, table 8). These mass features displayed variable intensity ratios in the necrosis patterns between REIMS spectra, including between patients (fig. 10c-d).

Table 6. Lipid identification by REIMS based on mass accuracy and tandem MS for the second hypothesis (tumour necrosis).

\begin{tabular}{|c|c|c|c|c|c|c|c|}
\hline Identification & Adduct & Formula & $\begin{array}{l}\text { PCA } \\
m / z\end{array}$ & $\begin{array}{c}\text { Measured } \\
\qquad \mathrm{m} / \mathrm{z}\end{array}$ & $\begin{array}{c}\text { Theoretical } \\
\qquad m / z\end{array}$ & $\begin{array}{l}\text { Mass } \\
\text { error } \\
\text { ppm }\end{array}$ & $\begin{array}{c}\text { Fragment } \\
\text { ions upon } \\
\text { MS/MS }\end{array}$ \\
\hline NEFA(16:0) & {$[\mathrm{M}-\mathrm{H}]^{-}$} & $\mathrm{C}_{16} \mathrm{H}_{31} \mathrm{O}_{2}$ & 255.25 & 255.23 & 255.23 & 1.8 & - \\
\hline NEFA(18:0) & {$[\mathrm{M}-\mathrm{H}]^{-}$} & $\mathrm{C}_{18} \mathrm{H}_{35} \mathrm{O}_{2}$ & 283.25 & 283.26 & 283.26 & 2.0 & - \\
\hline $\operatorname{Cer}(34: 1 ; 2)$ & {$[\mathrm{M}+\mathrm{Cl}]^{-}$} & $\mathrm{C}_{34} \mathrm{H}_{67} \mathrm{NO}_{3} \mathrm{Cl}$ & 572.45 & 572.48 & 572.48 & 2.1 & 536.5 \\
\hline $\operatorname{Cer}(42: 2 ; 2)$ & {$[\mathrm{M}+\mathrm{Cl}]^{-}$} & $\mathrm{C}_{42} \mathrm{H}^{81} \mathrm{NO}_{3} \mathrm{Cl}$ & 682.55 & 682.59 & 682.59 & 5.2 & 646.6 \\
\hline $\operatorname{SM}(34: 1 ; 2)^{* 1}$ & {$\left[\mathrm{M}-\mathrm{CH}_{3}\right]^{-}$} & $\mathrm{C}_{38} \mathrm{H}_{76} \mathrm{~N}_{2} \mathrm{O}_{6} \mathrm{P}$ & 687.55 & 687.54 & 687.54 & 2.1 & $168.0,79.0$ \\
\hline $\operatorname{SM}(42: 2 ; 2)^{* 1}$ & {$\left[\mathrm{M}-\mathrm{CH}_{3}\right]^{-}$} & $\mathrm{C}_{46} \mathrm{H}_{90} \mathrm{~N}_{2} \mathrm{O}_{6} \mathrm{P}$ & 797.65 & 797.65 & 797.65 & 4.1 & $168.0,79.0$ \\
\hline ACer(52:0;3) & {$[\mathrm{M}-\mathrm{H}]^{-}$} & $\mathrm{C}_{52} \mathrm{H}_{102} \mathrm{NO}_{5}$ & 820.75 & 820.77 & 820.78 & 2.9 & $\begin{array}{c}784.6,536.5 \\
283.3\end{array}$ \\
\hline $\begin{array}{l}\mathrm{Hex} 2 \mathrm{Cer}(34: 1 ; 2) \\
* 2\end{array}$ & {$[\mathrm{M}+\mathrm{Cl}]^{-}$} & $\mathrm{C}_{46} \mathrm{H}_{87} \mathrm{NO}_{13} \mathrm{Cl}$ & 896.55 & 896.58 & 896.59 & 2.5 & $\begin{array}{c}860.6,680.5 \\
698.5,536.5 \\
179.1\end{array}$ \\
\hline Acer(60:1;3) & {$[\mathrm{M}-\mathrm{H}]^{-}$} & $\mathrm{C}_{60} \mathrm{H}_{116} \mathrm{NO}_{5}$ & 930.85 & 930.88 & 930.89 & 4.3 & $\begin{array}{c}894.8,646.6 \\
283.3\end{array}$ \\
\hline $\begin{array}{l}\mathrm{Hex} 2 \mathrm{Cer}(42: 2 ; 2) \\
* 2\end{array}$ & {$[\mathrm{M}+\mathrm{Cl}]^{-}$} & $\mathrm{C}_{54} \mathrm{H}_{101} \mathrm{NO}_{13} \mathrm{Cl}$ & 1006.65 & 1006.69 & 1006.70 & 2.6 & $\begin{array}{c}970.7,790.6 \\
808.7,646.6 \\
179.1\end{array}$ \\
\hline \multicolumn{8}{|c|}{$\begin{array}{l}{ }^{* 1} \text { The demethylation, loss of }-\mathrm{CH}_{3} \text { is a common form reported for the identification of sphingomyelin referenced } \\
\text { in literature }(345,346) .{ }^{* 2} \mathrm{As} \text { mentioned on ALEX }{ }^{123} \text { lipid calculator (319), } \mathrm{m} / \mathrm{z} 896.6 \text { and } 1006.7 \text { species correspond } \\
\text { to lactosylceramide (Glc-Gal-ceramide) but the term dihexosylceramide is employed since MS cannot distinguish } \\
\text { the sugar moieties attached to the ceramide backbone. These mass features identified as dihexosylceramides are } \\
\text { assumed to be lactosylceramides, mainly reported in the literature, even if theoretically other sugar moieties } \\
\text { (other combination of hexoses) could be attached to the ceramide backbone. }\end{array}$} \\
\hline
\end{tabular}

Table 7. Lipid identification by DESI-MS based on mass accuracy and tandem MS providing informative fragmentation patterns of ceramide backbone corresponding to referenced publications (346,347).

\begin{tabular}{|c|l|l|c|c|c|c|}
\hline Identification & Adduct & Formula & $\begin{array}{c}\text { Measured } \\
\mathbf{m} / \mathbf{z}\end{array}$ & $\begin{array}{c}\text { Theoretical } \\
\mathbf{m} / \mathbf{z}\end{array}$ & $\begin{array}{c}\text { Mass error } \\
\text { (ppm) }\end{array}$ & $\begin{array}{c}\text { Fragment ions } \\
\text { upon MS/MS }\end{array}$ \\
\hline Cer 34:1;2 & {$[\mathrm{M}-\mathrm{H}]^{-}$} & $\mathrm{C}_{34} \mathrm{H}_{66} \mathrm{NO}_{3}$ & 536.50 & 536.50 & 0.8 & $\begin{array}{c}504.5,488.5,296.3, \\
280.3,263.2,237.2\end{array}$ \\
\hline Cer 42:2;2 & {$[\mathrm{M}-\mathrm{H}]^{-}$} & $\mathrm{C}_{42} \mathrm{H}_{80} \mathrm{NO}_{3}$ & 646.62 & 646.61 & 2.4 & $\begin{array}{c}616.6,598.6,406.4,390.4, \\
347.3,263.2,237.2\end{array}$ \\
\hline
\end{tabular}




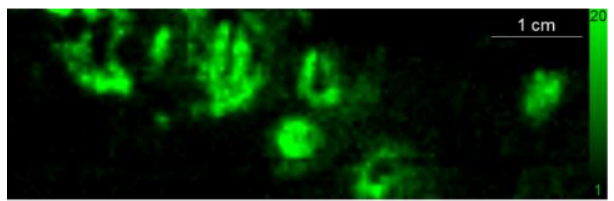

Ceramide $34: 1$

$\mathrm{m} / \mathrm{z} 536.50[\mathrm{M}-\mathrm{H}]$

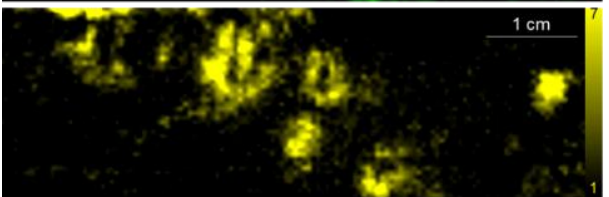

Ceramide $42: 2$

$\mathrm{m} / \mathrm{z} 646.62[\mathrm{M}-\mathrm{H}]$

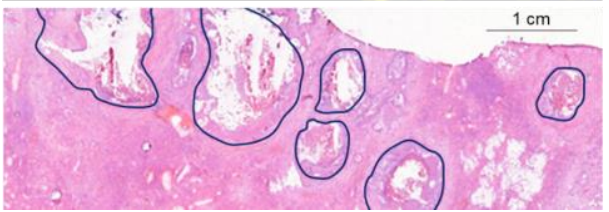

Figure 11. Molecular distribution of ceramide $34: 1(\mathrm{~m} / \mathrm{z} 536.50)$ and ceramide $42: 2(\mathrm{~m} / \mathrm{z} 646.62)$ in necrotic areas surrounding calcifications of a frozen section of MAC (colorectal metastatic adenocarcinoma) at $50 \times 50 \mu \mathrm{m}^{2}$ pixel size by DESI-MSI.

Table 8. P-values (t-tests) of mass features discriminative of necrosis from viable MAC.

\begin{tabular}{|c|c|c|c|}
\hline Mass feature & P-value & Mass feature & P-value \\
\hline 255.25 & $6.91 \times 10^{-12}$ & 797.65 & $1.81 \times 10^{-28}$ \\
\hline 283.25 & $3.78 \times 10^{-12}$ & 820.75 & $1.64 \times 10^{-12}$ \\
\hline 572.45 & $1.31 \times 10^{-26}$ & 896.55 & $1.09 \times 10^{-21}$ \\
\hline 682.55 & $9.45 \times 10^{-28}$ & 930.85 & $5.78 \times 10^{-24}$ \\
\hline 687.55 & $7.49 \times 10^{-15}$ & 1006.65 & $8.18 \times 10^{-21}$ \\
\hline
\end{tabular}
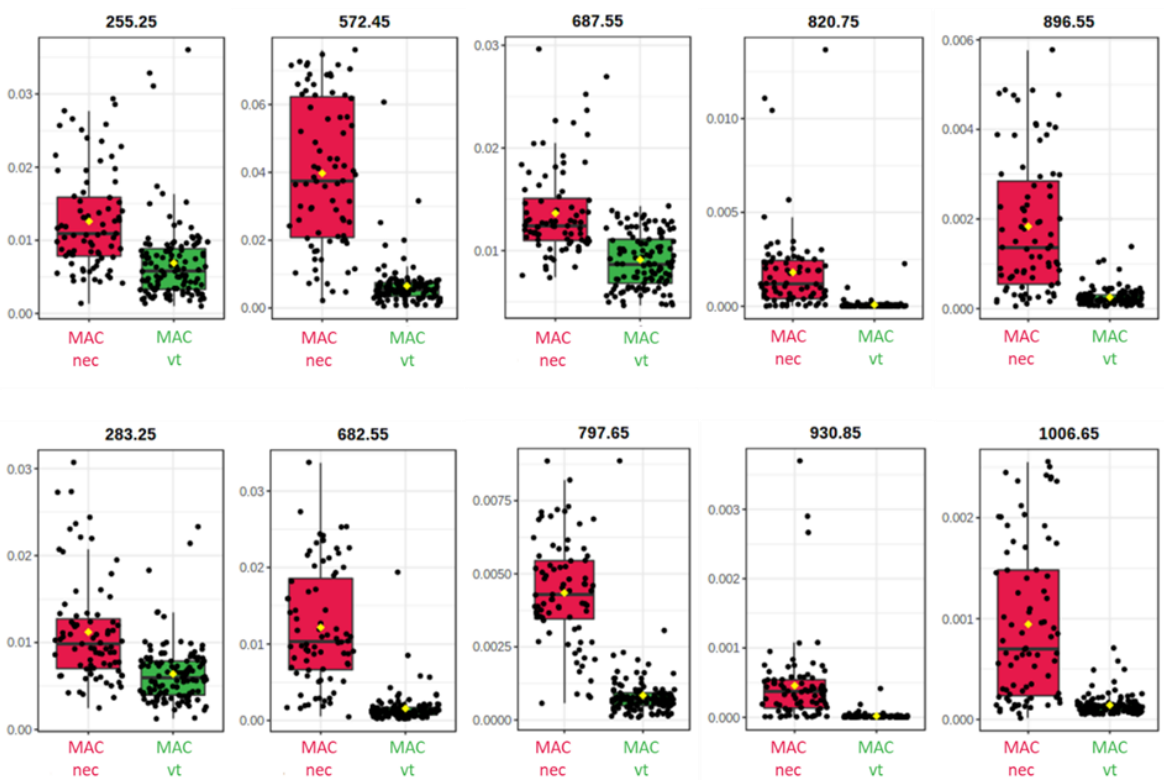

Figure 12. T-tests for mass features to discriminate MAC necrosis. Graphical summary. 


\section{Classification of viable and necrotic tumour of different human tumours}

As ceramides and related sphingolipids discriminated necrotic MAC from viable MAC in diverse intensity ratios, it was of interest to establish whether the identified mass features in colorectal metastases are tumour type specific or can also be found in the necrotic part of other tumour types. Therefore, the lipid patterns were compared between viable and necrotic tumour in $1 \mathrm{HCC}, 1$ liver metastasis of a neuroendocrine tumour, 1 extrahepatic liposarcoma and 1 extrahepatic adrenocortical carcinoma. Results are shown in figure 13. On the PCA score plots, each tumour displayed a separation of viable and necrotic tumour along their PC1 axis. All PC1 loading plots indicated mass features discriminative of necrotic tumour similar to what was observed in necrotic MAC (fig. 10c). However, the intensity ratios of these mass features differed between tumours (PCA mass features loading plots in fig. 13).

Noteworthy, a lipid pattern different from the ceramide pattern to discriminate necrotic from viable tumour in two other HCC and stomach HCC metastasis was also observed. Details can be found in the next paragraph and in figure 14.

a)

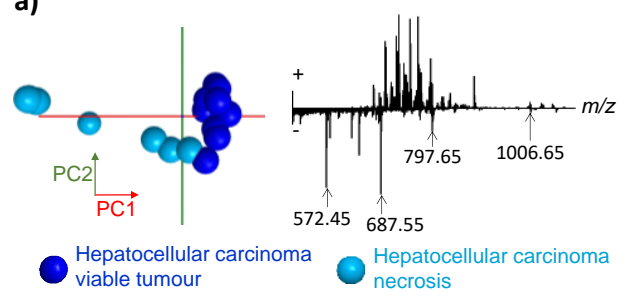

c)

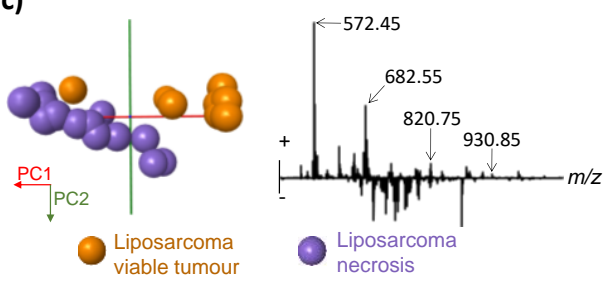

b)

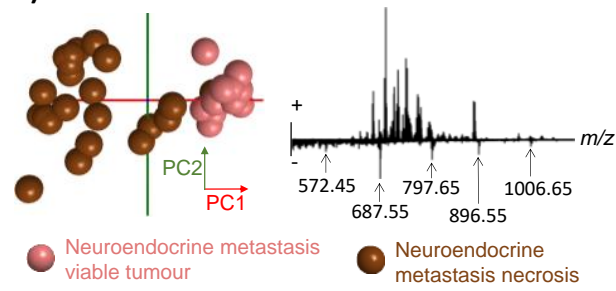

d)

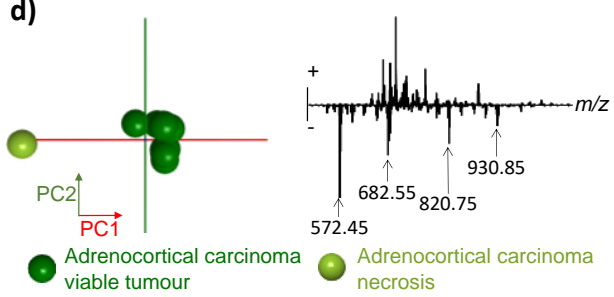

Figure 13. Discrimination between necrotic and viable tumour. (a-d) Principal component analysis (PCA) score plots on the left and respective PC1 mass features loading plots on the right (mass range $m / z$ 500-1100) for:

(a) 19 lipid patterns (13 viable, 6 necrotic) generated from a hepatocellular carcinoma.

(b) 41 lipid patterns (18 viable, 23 necrotic) generated from a neuroendocrine tumour liver metastasis.

(c) 23 lipid patterns (9 viable, 14 necrotic) generated from an extrahepatic liposarcoma.

(d) 9 patterns (8 viable, 1 necrotic) generated from an extrahepatic adrenocortical carcinoma.

\section{Heterogeneous lipid patterns to classify necrosis and viable tumour parts in hepatocellular carcinomas}

The study was then focused on 4 HCC cases that include necrotic patterns. Group 1 (fig. 14ac) is the result of 2 cases, group 2 (fig. 14d-g) is the result for 2 other cases. For both groups, PC1 axis separates HCC viable tumour and HCC necrosis patterns (fig. 14a,d). For group 2, 
the patterns of the stomach HCC were included in the PCA score plot. Intensity changes were observed between the primary HCC necrosis and the stomach metastatic necrosis patterns of the same patient, but not for the viable tumour patterns (fig. 14f-g).

Respective PC1 loading plots displayed distinctive mass features for necrosis (fig. 14b and 14e). The pattern of the group 1 (fig. 14b) included mass features similar to the ones of MAC necrosis (fig. 10c).

A PCA score plot including HCC necrosis and MAC necrosis targeted on two main mass features of PC1 of both HCC necrosis patterns displayed a separation of two groups (fig. 14h-i). This confirmed the existence of two distinctive necrosis patterns in HCC, one being similar to the MAC necrosis pattern (fig. 14a-c).

a)

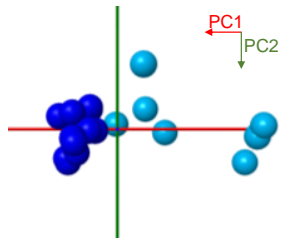

d)

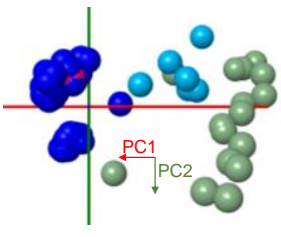

b)

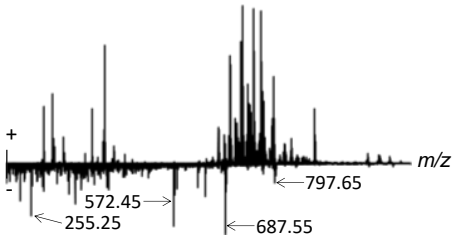

e)

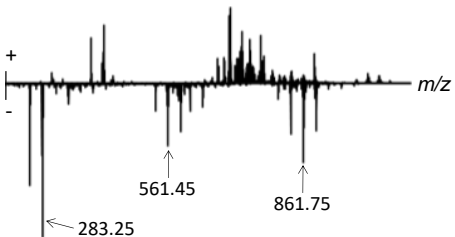

c)

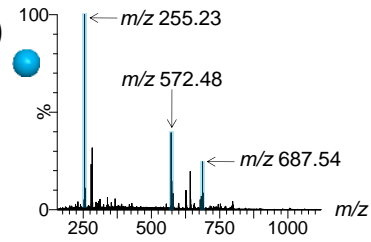

f)

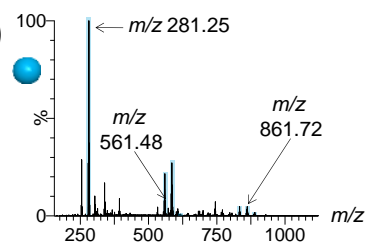

h)

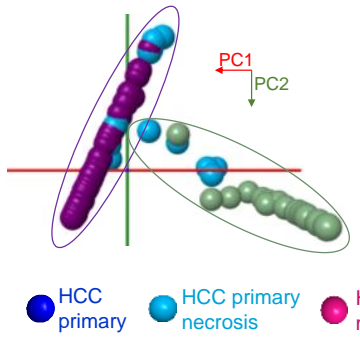

i)

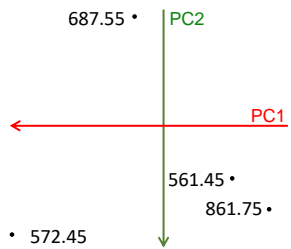

g)

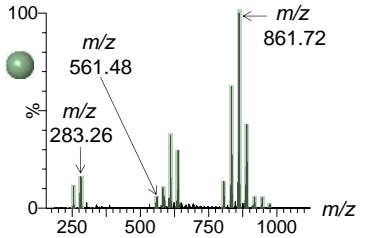

Figure 14. Heterogeneous lipid patterns to discriminate necrotic from viable tumour in hepatocellular carcinoma (HCC).

(a) Principal component analysis (PCA) score plot of $20 \mathrm{HCC}$ patterns (13 viable tumour, 7 necrosis) from 2 patients (PC1 70.3\%, PC2 8.4\%, mass range $m / z$ 200-1100).

(b) PC1 mass features loading plot.

(c) One HCC necrotic pattern.

(d) PCA score plot (PC1 52.3\%, PC2 17.0\%, mass range $m / z$ 200-1100) of HCC patterns (105 viable from liver primary site, 6 necrotic from liver primary site, 5 viable from stomach metastasis, 16 necrotic from stomach metastasis) from 2 other patients.

(e) PC1 mass features loading plot.

(f) Single HCC necrotic pattern from the primary site from one patient.

(g) Single HCC necrotic pattern from the stomach metastatic site of the same patient as (F).

(h) PCA score plot of necrosis patterns from the 4 HCC cases above and the MAC cohort (PC1 63.2\%, PC2 28.8\%) targeted on 4 mass features discriminative of the 2 HCC necrotic patterns: 561.45, 572.45, 687.55, and 861.75.

(i) Respective PC1/PC2 mass features loading plot. 
One other HCC case had inconsistent separation of viable tumour and necrosis patterns. Histologically, necrotic tissues of this case were particularity bloody. No discriminative mass features for necrosis appeared obvious and this case was left out the analysis.

\section{Comparisons of necrotic tumours targeted on ceramide metabolic shifts}

As the same mass features identified as ceramides and related sphingolipids discriminated necrotic from viable tumour in multiple different human tumours, the comparison of their intensity ratios in necrotic patterns between tumours was investigated. The potential of ceramide metabolism from necrotic areas was explored for patient sub-classification, independently from the tumour type.

PCA score plots of necrotic tumour lipid patterns targeting 4 specific mass features were used to evaluate the impact of metabolic shifts involving 2 ceramides with 2 acylceramides (fig. 15a-b), 2 lactosylceramides (fig. 15c-d) or 2 sphingomyelins (fig. 15e-f). Metabolic links between the mass features used for the PCA were integrated into each PC1/PC2 loading plots (fig. 15b, 15d, 15f) and can be seen in an overview of the sphingolipid pathway in figure 16 .

The orientation of the arrows linking each ceramide and their respective metabolic partner differed. Parallel arrows were displayed only between each ceramide and respective sphingomyelin (fig. 15i), neither for acylceramides (fig. 15c) or for lactosylceramides (fig. 15f). Only ceramides and sphingomyelins were linked by a single and direct biochemical reaction.

The necrotic MAC lipid patterns of one of the patients appeared to be more similar to the necrotic tumour lipid patterns of the adrenocortical carcinoma (ACC) and the liposarcoma (LPS) based on the balance ceramides/acylceramides (fig. 15a). Similar observation was made on the metabolic shift ceramides/lactosylceramides for another MAC patient closer to the neuroendocrine tumour (NET) (fig. 15d). For the ratio ceramides/sphingomyelins, MAC and LPS necrotic patterns were more heterogeneously distributed compared to the necrotic NET patterns (fig. 15g).

\section{Discussion}

In current clinical practice, intraoperative decisions often depend on ex vivo examination of resected tissue by a pathologist using frozen tissue sections. Technical issues such as size of the tissue piece that can be investigated during frozen section pathology, type of material (some tissue types cannot be cut frozen) and time constraints during surgery can make frozen sections challenging to interpret with certainty. Besides, the diagnostic delay between excision time and reported diagnosis may hamper the judgement regarding the extension of the resection. Completeness of tumour resection during surgery is usually evaluated by histopathological examination of the fixed resected specimen after surgery which takes several days. In addition, instant diagnosis of malignancy can be challenging if relevant morphological features are not readily recognizable. Diagnostic subjectivity (19) can lead to disagreement between experts (20). For definitive diagnosis, histopathology 
may need to be complemented by immunohistochemistry or other molecular investigations to enable the identification of the origin of the tumour extending the specimen reporting time (351).

a)

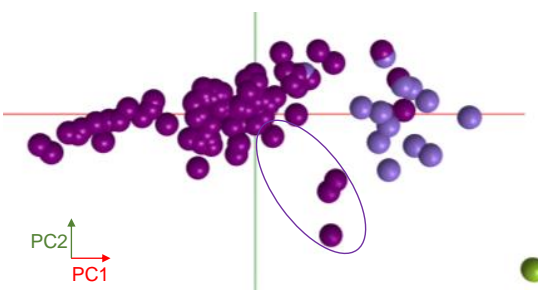

c)

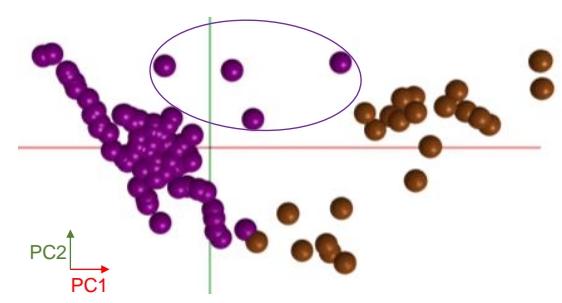

e)

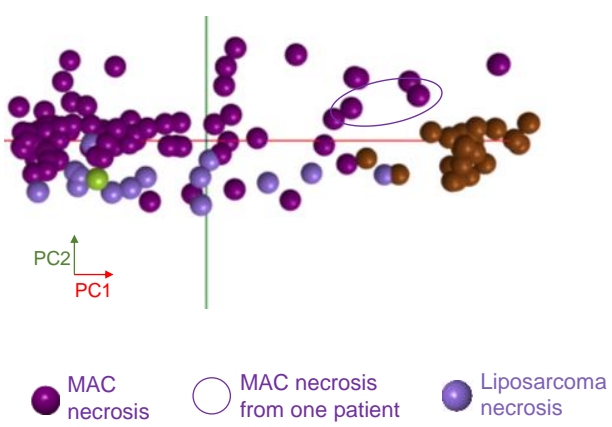

b)

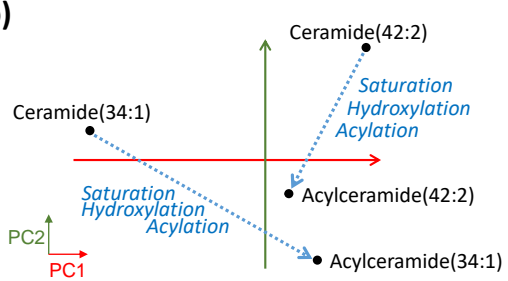

d)

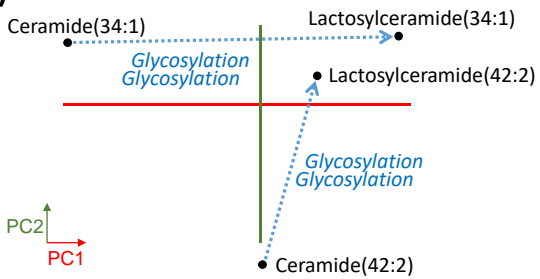

f)

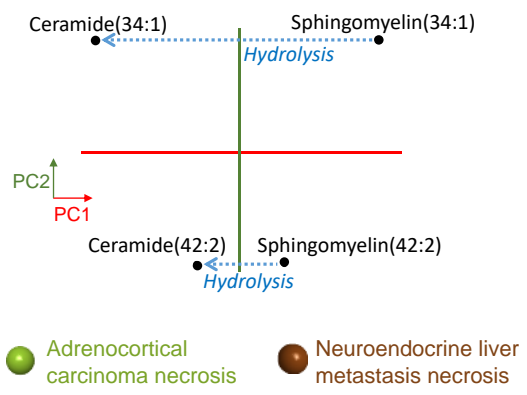

Figure 15. Comparisons of necrotic tumours based on ceramide metabolic shifts. (a, c, e) Principal component analysis (PCA) score plots, $(\mathbf{b}, \mathbf{d}, \mathbf{f})$ their respective PC1/PC2 mass features loading plots.

(a) PCA score plot of 94 necrosis patterns (79 MAC, 14 LPS, 1 ACC) targeting 4 mass features assigned as ceramides $(572.45,682.55)$ and acylceramides $(820.75,930.85)$, generated from tissues of 16 patients (14 MAC, 1 LPS, 1 ACC; 5 PC dimensions, PC1 88.6\%, PC2 11.2\%).

(c) PCA score plot of 102 necrosis patterns (79 MAC, 23 NET) targeting 4 mass features assigned as ceramides $(572.45,682.55)$ and lactosylceramides $(896.55,1006.65)$ generated from tissues of 15 patients (14 MAC, 1 NET; 5 PC dimensions, PC1 86.0\%, PC2 13.8\%).

(e) PCA score plot of 117 necrosis patterns (79 MAC, 1 ACC, 14 LPS, 23 NET) targeting 4 mass features assigned as ceramides $(572.45,682.55)$ and sphingomyelins $(687.55,797.65)$ generated from tissues of 17 patients (14 MAC, 1 NET, 1 LPS, 1 ACC; 5 PC dimensions, PC1 95.4\%, PC2 3.7\%).

MAC: metastatic adenocarcinoma; LPS: liposarcoma, ACC: adrenocortical carcinoma, NET: neuroendocrine tumour

Near-real time feedback to guide surgical decisions could potentially improve patient care and let to the investigation of fresh tissue classifiers based on lipid patterns. The potential 
of lipid patterns for the classification of liver tumour tissues has already been suggested using mass spectrometry imaging (MSI) $(27)$ on frozen sections $(352,353)$ but also rapid evaporative ionization mass spectrometry (REIMS) of electrosurgical vapours (29) which allows analysis on fresh resected tissues and a real-time feedback. The classification of normal liver parenchyma (LP), hepatocellular carcinoma (HCC) and liver metastasis of colorectal and breast cancer (MAC) based on ex vivo real-time lipid patterns reached $98.1 \%$ accuracy when compared to gold standard pathology suggesting that REIMS technology may be able to assist liver tumour identification during surgery.

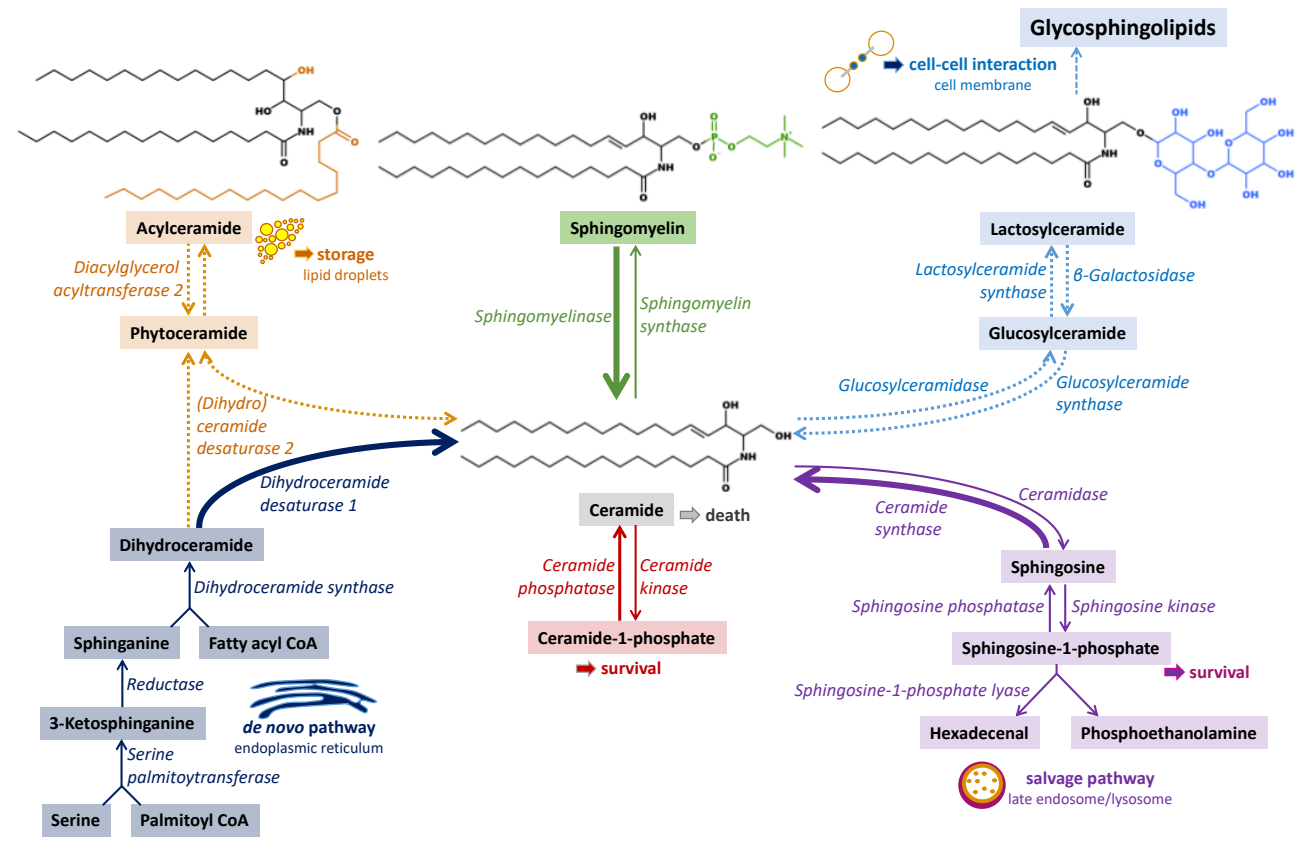

Figure 16. Scheme of sphingolipid metabolism. Integration of the ceramides and related sphingolipids discriminative of tumour necrosis detected by REIMS with apparent molecular structures (represented with ceramide backbone d18:1/16/0). Scheme adapted from referenced literature (334,354-358).

Similarly, the investigation demonstrates that lipid patterns provide immediate insights of the tumour metabolic phenotype, which may improve clinical decision. The experiment was performed ex vivo with a surgical hand-piece but dedicated tools using less destructive sampling with REIMS or other ionization sources also generating lipid patterns have provided similar conclusions $(30,31,306,359)$. Likewise, rapid MSI analyses of frozen sections could provide an assessment of the tissue malignancy in a compatible timeframe with intraoperative diagnostics, with the benefit of precise histological correlation $(360,361)$. These mass spectrometry technologies based on real-time lipid patterns recognition for cancer precision medicine could be implemented in the operating room or in the pathology laboratory, depending on the impact of the results for clinical decisions. Characterization of tumour heterogeneity could influence clinical interventions (e.g. 
chemotherapy selection) but could also be conceived as impactful for surgical decisions such as optimized margin resection based on the tumour biology. These perspectives of cancer precision required collaborative efforts for validation of the findings in prospective study on more tissue pieces and investigations about feasibility and implementation. Lipid patterns could translate findings from other research fields such as genomics or proteomics, or correlate directly with detailed clinical data. Here, this work illustrated how lipid patterns can characterize tumour hallmarks on two hypotheses regarding metastasis and necrosis.

For the first hypothesis, the overview of the variance of the lipid patterns was interpreted with regards to tumour heterogeneity and histopathology. While HCC patterns were substantially different in-between patients (intertumour heterogeneity), MAC patterns were more similar in-between patients (fig. 7b). The homogenous patterns obtained from MAC compared to the heterogeneous HCC patterns (fig. 7a) goes along with the biological variance expected when comparing primary and secondary tumours. Secondary tumours, as MAC, share common ground biological characteristics to leave their initial generating site, migrate, attach and grow in a new tissue environment (340). HCC generate more diverse clones of tumours in their initial liver site with multiple features of growth patterns and dedifferentiation with loss of hepatic healthy architecture (fig. 7c), whereas MAC are generally homogenous and well differentiated in the liver (fig. 3d).

The generation of patient specific tumour patterns specific for each patient can be used to evaluate the respective impact of inter-and intratumour heterogeneity on clinical decisions. Further research investigations could contribute to a better understanding of tumour heterogeneity assessment by lipid patterns. The precise spatial distribution of lipids in tissue sections with mass spectrometry imaging (27) complemented by morphometric analysis (362) represents one of the leads to detail the links between lipid patterns and histopathology changes. It demonstrated that lipid patterns generated by REIMS can provide a fast and quantifiable modality to complement histopathology in the assessment of tumour heterogeneity.

For the second hypothesis, the discrimination of viable and necrotic parts of MAC $192.9 \%$ accuracy) and other tumours was studied to identify specific lipids involved in tumour cell death metabolism. These lipids identified as ceramides and related sphingolipids were targeted to classify patient tumours based on specific metabolic shifts, which can be a challenging approach for real-time classifiers.

Real-time classifiers present analytical limitations in comparison to the comprehensive characterization of lipid extracts after chromatographic separation (363) for targeted analysis. The detection of lipid species that contribute to the classifier, identification confidence and their biological interpretation are compromised by the presence of isobaric or isomeric complex lipids, in-source fragmentation or the lack of resolving power during mass spectrometric analysis. Even if online databases of mass spectra and previous publications generated on diverse platforms are used to confidentially validate lipid 
identifications, the biological roles of lipids is only partly understood. The still growing knowledge of the role of lipids in tumour biology (334) and the interconnected metabolic pathways each species may experience, also represent limitations of biological interpretation. A multitude of diverse and interconnected metabolic pathways are simultaneously active in viable cellular tissues. This makes them more challenging to isolate from each other by mass spectrometry. Before cell-death, metabolism might be directed towards essential functional activities, leading to "simpler" necrosis patterns more suitable for investigation. The simultaneous detection of lipids that are metabolically closely related, and their statistically significant contribution to discriminate tissues confirm that our findings depict a real-time biological reality.

Moreover, the report of a ceramide pattern discriminative of tumour necrosis by REIMS correlates with previous investigations where ceramides were identified in situ by MSI (27) in necrotic areas of human tumours using diverse analytical platforms. Tata et al. highlighted Cer(34:1) in tissue sections of a variant of a breast cancer cell line xenograft in mice (364). The authors suggested $\operatorname{Cer}(34: 1)$ as shared marker for necrosis among various tumours, supported by previous studies on glioblastomas (365). In addition, Cer(34:1) was reported as part of a lipid pattern in colorectal liver metastasis from 50 patients to distinguish usual necrosis, typical of tumour progression, from infarcted-like necrosis, typical of response to Bevacizumab. Similarly, the distributions of both $\operatorname{Cer}(34: 1)$ and Cer(42:2) characterized necrotic areas of high grade serous ovarian cancers (366).

The precise metabolic interconnections of these ceramides and related sphingolipids in tumour cell death are critical to account for and useful to identify targets for cancer therapies. Ceramides are central metabolites of the sphingolipids metabolism (fig. 16) and their balance with related sphingolipids is critical in the fate of cells for growth and survival (367). A multitude of cell stress responses to stimuli such as tumour necrosis factor or ultraviolet radiations induce accumulation of ceramides, leads to a cascade of events that involve activation of caspases and permeabilization of mitochondrial membranes with release of cytochrome $C$ to induce cell death $(341,342)$. Ceramides can be generated by sphingomyelinase, through de novo or salvage pathways, and their accumulation acts in synergy with chemotherapeutic agents to increase tumour cell death. Pathways that consume the pool of ceramides can be considered pro-survival processes to protect again cell death, enhance resistance to treatment, and contribute to cancer progression $(335,368)$.

Acylceramides were recently considered a storage form of ceramides in lipid droplets to regulate their accumulation (356). Increase of acylceramides in colon adenocarcinoma cell lines was associated with more cell survival, and therefore resistance to chemotherapy. Structurally, the backbone of the acylceramides in the present investigation was assigned to a phytoceramide, limitedly reported in literature $(355,357,358)$. An extra hydroxylation and desaturation step in the production of acylceramides from ceramides appears plausible: the extra hydroxyl in the sphingosine backbone was suggested to enable lipid packing in the membrane through an increased amount of hydrogen bounding at the 
membrane interface (354). Thus, the storage of acylceramides as phytoceramides seems structurally and biologically rational.

Similarly, the balance between ceramides and lactosylceramides, and glycosphingolipids in general, has been topic of interest in drug therapy, in particular associated to the overexpression of efflux pump P-glycoprotein (369). Glucosylceramide synthase (GCS) balances between pro-apoptotic ceramide and anti-apoptotic glucosylceramide is therefore the object of studies about drug resistance (343) and drug injury (370).

The delicate balance ceramides/sphingomyelins is indicative of a direct and single biochemical reaction, critical in cell death: the hydrolysis of sphingomyelins into ceramides by sphingomyelinase. In addition to a difference in the phase of the necrosis process, the heterogeneity of this metabolic balance between tumours could be associated to two hypotheses. First, some tumours could require more accumulation of ceramides to die than other tumours. Second, some tumours could generate the ceramides mainly by the sphingomyelinase while other tumours could mainly use the de novo or the salvage pathways.

Moreover, the presence of sphingomyelin as a discriminator of necrosis from viable tumour parts could suggest that the sphingomyelin breakdown might not be consistently substantial in the generation of ceramides. With regards to patterns changes interpretation, the relative content of the tissue lipid pattern could shift from glycerophospholipids, main structural lipid class reported by REIMS $(29,302,315)$, to sphingolipids during the onset of necrosis. The last catabolic break-down might lead to the accumulation of remnant endproduct lipids that mainly represent the patterns of dead tumour cells. In the absence of functional ceramidases, probably after lysosomes lysis, the ceramide backbone remains intact. Therefore, ceramides and related sphingolipids accumulate in necrotic tissue due to their lipophilic nature that hampers their diffusion (354). In opposition, the degradation of glycerophospholipids by enzymes such as phospholipases, or spontaneous hydrolyses, could explain the production of fatty acids that are more prone to diffusion.

Yet, the ceramide pattern is not to be considered a universal pattern for the detection of necrosis in human tumours because a lipid pattern discriminative of necrosis different from the ceramide pattern (fig. 10, 13, 14) in two HCC cases (fig. 14) was observed. Nevertheless, it appears independent from the tumour origin, whether the tumour originates from ectodermal (i.e. for MAC, HCC) or mesodermal (i.e. LPS, ACC) cells/tissues (371,372).

Additionally, relative intensity changes of the ceramides in the pattern could provide tumour characteristic molecular information reachable in real-time. In the investigation on metabolic shifts, acylceramides were dominant in LPS and ACC necrosis patterns compared to the MAC cohort (fig. 15a-b). Pharmacokinetics considerations aside, these results appear compatible with the moderate effect of cytotoxic agents to treat these tumours $(373,374)$. Likewise, the MAC necrosis patterns of one patient presented predominant acylceramides compared to the rest of the MAC cohort. It can be hypothesized that the tumour of this patient could react differently to treatment than the rest of the cohort. Similar substantial metabolic shift was noticed between ceramides and lactosylceramides (fig. 15c-d) 
suggesting that the full viable tumour might be different metabolically, even if not histologically. As these metabolic shifts do not appear associated to local intratumour heterogeneity, this kind of findings could have an impact for clinical decision.

This study provides evidence that knowledgeable insights can be obtained from the analysis of necrotic tissues, while the morphologic information to be extracted by a pathologist from a necrotic tissue remains subtler than from cellular tissues. The variety of lipid patterns in tumour necrosis could be associated with a different phase or specificity of the metabolic process to be reliable to provide insight about the metabolism of the viable tumour. In addition, the common detection of necrosis by the same ceramides enables comparisons of tumours from different types based on specific metabolic shifts. It opens new perspective towards translating findings from frequent cancers to rare cancers, often marginally studied, to also improve their clinical management.

\section{Conclusion}

In conclusion, lipid patterns generated by REIMS can provide a fast and quantifiable modality to identify and characterize tumour tissue and its heterogeneity. Moreover, a realtime analytical technique has the ability to reflect cell death, one of the most significant biological processes involved in human diseases. The translational aspect of these findings strengthens the scientific knowledge gathered on lipid metabolism during tumour cell death. The versatility of REIMS allows metabolic analysis of cell models (297) through biological tissues during surgery. In a field governed by tumour heterogeneity affecting patient outcome, precise characterization of heterogeneous tumour metabolic phenotypes is expected to transform clinical decision-making. 
Chapter 6

Valorisation 


\section{The surgical resection and the reference}

Cancer is a leading cause of human death worldwide (1). The treatment of patients with cancer has tremendous repercussions on their quality of life and oncologic surgery is one of the most critical steps of the treatment. Surgeons aim to remove the full tumour from the patient's body while sparing healthy tissue to maintain functions. Intraoperative decisions on the execution of the surgical resection are critical for patient outcome (9-11) and unsuccessful surgeries can lead to tumour recurrence, and therefore, patients may have to undergo reoperation or heavy complementary treatments.

One of the intraoperative challenges is the macroscopic recognition and localisation of tumour tissues by the surgeons. Preoperative, perioperative and postoperative guidance modalities can assist surgeons to localise the tumour in the tissues $(12,16,17)$. Among these modalities, histopathology, the reference for the diagnostic of tumour tissues, can assist surgeons during and after the operation.

Histopathology examination required a multi-step tissue process. Laboratory technicians have to prepare the tissue specimen for histology with fixation, sectioning and staining and then, trained pathologists have to recognize histological features indicative of the disease of the tissue under a microscope. This involves a substantial infrastructure with a laboratory to process tissues, professional experts specialised in their own their pathology discipline. Nevertheless, the subjective interpretation of the tissue sections can lead to divergence in diagnostics between experts.

For perioperative histopathology examination, the tissue specimen is frozen (e. g. in liquid nitrogen) due to the time constraint requested feedback. Pathology examination of frozen sections can be a difficulty due to the quality of the tissue fixation that alters the histological features. For postoperative histopathology examination, the full surgically resected specimen is sent for pathology examination. Tissue is fixed by immersion in formalin for hours due to the rate of penetration of formalin in the tissue. The pathologist selects parts of the tissue for microscopic examination and elaborates a report to provide feedback about the success of the surgery. The elaboration of the report can about a week.

There is a need to assist surgeons for more precise, more reliable surgical executions with providing fast assessment of the disease of the tissue during the surgery.

\section{The challenger vs. the reference}

Rapid evaporative ionization mass spectrometry (REIMS), a new ionization source that enables the recognition of tissues based on lipid patterns was employed in the investigation presented in this thesis. Adapted to the classic surgical tools, REIMS can analyse directly the composition of surgical vapours and aerosols generated during surgery and provides feedback in seconds. While current perioperative pathology assessment requires dedicated instruments and trained professionals for tissue processing and examination, REIMS could provide an on-line, real-time pathology assessment while the surgeon is performing the 
surgery. The benefit of the REIMS technology for intraoperative tissue recognition appears very appealing to improve surgical precision.

As for any new diagnostic test, a comparison to the reference diagnostic is required to show the accuracy of its performance. Therefore, all the human tissues analysed with REIMS were also subsequently processed for optimal histology and pathology validation to prove the reliability of the REIMS technology for pathology assessment. The accuracy of tissue classifications based on lipid patterns generated by REIMS were compared to the tissue type assessed by the pathologist. The models reached accuracy above $90 \%$ accuracy for the distinction of normal and tumour tissues, which prove the performance of the technology.

\section{Get in}

Resected tumour tissues from patients undergoing surgery at Maastricht University Medical Centre were collected during the pathology examination of the resected specimen. Right after, tissue slices were directly analysed by REIMS in the laboratory. A library of molecular patterns was established with pathology validation based on these ex vivo analyses. The generated models could recognize tissue in the same ex vivo context. However, a change of metabolic activity could influence substantially the molecular pattern between in vivo and ex vivo tissue analyses. Nevertheless, this model generated ex vivo enabled recognition of tissue types during breast conserving-surgery showing the proof that an ex vivo generated model can recognize in vivo tissue types (chapter 4). The translation from ex vivo to in vivo is of particular interest regarding the previous accomplishments performed by mass spectrometry imaging on clinical studies (chapter 2 ).

\section{Beyond the reference}

The goal of the implementation of the REIMS technology in surgical environments is to assist surgeons with tumour resection for more precise surgery. REIMS classification results were validated by histopathology, the reference for tumour diagnostics. Interestingly, the results generated by REIMS and DESI-MS also showed a change of molecular patterns between tumour border stroma and remote tumour stroma in the application on breast pathology (chapter 4). These results suggest that beyond the reference histopathology validated margin of resection, a molecular margin may exist and could be used to guide even more precisely surgeons. This constitutes a real step forwards to prove the relevance of molecular patterns for surgical guidance.

\section{Beyond the surgical resection}

The real-time analysis represents a significant novelty for the recognition of tissue types based on molecular patterns. Mass spectrometry imaging (MSI) has classified tissue types from frozen or formalin fixed samples for years. Molecular patterns based on small metabolites, lipids, trypsin-digested peptides, glycans or proteins enabled relevant classifications for clinical applications such as tumour typing, treatment response and 
overall survival (chapter 2). The fact that molecular patterns can be reached in real-time and even in vivo questions on how relevant the extracted information from these rapid analyses can be. Precision medicine can benefit from fast tumour metabolic phenotype identification.

Tumour heterogeneity is one of the biggest hassles of cancer treatment. Cancer development is a multistep process stimulated by oncogenic events specific to each patient due to their individual hereditary background and their individual environmental exposure. Moreover, tumour can develop multiple clones each presenting specific biological abilities to metastasis or response to treatment. These inter- and intratumour heterogeneity could be depicted in their metabolic phenotype.

The classification of liver tumours and tumour necrosis based on lipid patterns was studied (chapter 5). It showed that the performance and that the heterogeneity of the lipid patterns could be associated to histopathological changes. Patient specific tumour patterns could be generated to help decision-making. Moreover, a common lipid pattern to discriminate necrosis from viable parts in multiple human tumours was reported. The main discriminators of this necrosis patterns were ceramides and relative intermediates, wellknown bioactive lipids for the study of cell death. Targeted analyses on the necrosis patterns based on metabolic shifts led to new perspectives for tumour classification. This investigation showed that real-time molecular patterns can deal with other clinical applications than the only the surgical resection such as tumour heterogeneity. These findings indicate that lipid patterns may have the potential to guide clinical interventions such as drug therapy based on tumour metabolism.

\section{Innovative reproducibility}

Mass spectrometry started classifying tissues based on molecular patterns 20 years ago. The field has been driven by technological improvements. The consideration for the biological applications has been limited by the non-existence of reproducibility studies in an MSI field where optimisation of sample preparation and acquisitions are study-dependant. The emerging clinical applications led to the first multicentre studies on breast cancer, one of the most prevalent cancers worldwide (chapter 2).

This thesis includes the work on the first multicentre study on the reproducibility of REIMS for the classification of biological tissues (chapter 3 ). This study included breast tissues with histopathology validation. REIMS does not require sample preparation, which is an advantage for comparative studies. Moreover, a common methodology was established and key parameters were identified for fair comparison of the library of each site. Quality controls were included on each site to confirm the similar performance of the instruments and repeatability measurements for food items. This study constitutes a major advance in the applications of mass patterns towards their implementations in clinical practice. 


\section{Beyond research purpose only}

The investigations reported in this thesis contribute to bring closer to the operating room the molecular patterns-based tissue classification to support decision-making and enable more precise surgical resections. One of the most atypical achievement of this work was the record of electrosurgical vapours in vivo during cancer surgeries. These experiments performed on a mobile mass spectrometer required a specific organization with a dedicated patient consent form to participate to the research study and the respect of a safety protocol to fit the risk assessment in place in operating rooms. Information collected from these experiments remained for research purpose only, surgeons were not allowed to adapt their resection to it. Substantial steps remain before the potential use of such technology, including the assessment of its clinical benefit.

The diagnostic use of REIMS combined with the surgical tools based on the analysis of in vivo electrosurgical vapours represents a new paradigm in the field of diagnostics devices, and therefore a challenging regulatory framework. It does neither constitute a classic in vivo diagnostic test (e. g. administration of drug, isotope complemented with imaging modalities) or an ex vivo diagnostic test (performed on materialized biological samples taken from the human body). However, ex vivo testing of tissue constitutes an in vitro diagnostic test and appears convenient as a first approach to reach its regulation with European and American agencies (European Medical Agency and Food and Drug Administration).

The hospital framework with in vivo measurements, the very heterogeneous tissue pathologies and the establishment of tissue libraries with detailed histopathology validation for each tissue sampling are among the challenges of the surgical application. In addition, the advantage of this technology needs to be proven beneficial for patient's outcome with prospective clinical trials which may delay research progress towards diagnostics use of real-time tissue classifiers for histopathology assessment.

The surgical application of real-time tissue classifiers has represented and is still one of the strongest and exciting drive of the mass spectrometric classifiers over the recent years. The investigation of cancer physiopathology with in situ molecular patterns to translate gold standard histopathology diagnostics in real-time to improve surgical margin resections constitutes a clinical research field of high interest, and benefit from a clear message to be communicate to a general audience. Nevertheless, on short term, other applications appear already more rentable that the surgical application, with the benefice of robust comparative diagnostics tests and more easily built lipid patterns libraries using high-throughput robotic platform. It is the case for the classifications of microorganisms (293-296) and food items (288-292), and therefore, microbiology and food fraud represent more convenient applications to show the reliability of the technology and reach more accessible markets. These applications may drive the improvement of the technology with high-scale comparative studies over the coming years, and benefit to the development of the surgical application. 

Chapter 7

Summary and outlook 


\section{Summary and Outlook}

Oncologic surgery is a keystone step in the management of patients with cancer. It aims to remove all the tumour tissue to cure the patient from cancer, while sparing healthy tissues to preserve functions. While intraoperative decisions are critical for patient's outcome (911), on-spot macroscopic identification of tumour or healthy tissues can be difficult for surgeons. This intraoperative challenge has driven the development of real-time mass spectrometry technologies such as rapid evaporative ionization mass spectrometry (REIMS) analysis of electrosurgical vapours, to classify tissues in the operating room. It provides histopathological assessment to efficiently support surgical decisions based on molecular patterns. This work supports the vision that REIMS technology can be implemented into surgical environments around the world for routine margin assessment during cancer surgery.

First, this thesis reviewed the state of the art of the research field of tissue classification based on mass spectrometric patterns. Then, the reproducibility of the REIMS technology was assessed towards its reliability for tissue diagnostics in a multicentre study. Finally, the potential of REIMS was reported to support surgical resection on breast tissues and in vivo, and to characterise on-site tumour heterogeneity on liver tumour tissues. This thesis elaborates on their respective implications, and suggests investigations to pursue and expend this line of research. In addition, the lipid patterns are particularly discussed as bases of these classifiers to reflect their underlying tissue pathobiology.

\section{Mass spectrometry imaging, contributions and directions}

Chapter 1 reviewed the milestones of mass spectrometry imaging (MSI) that have led to consider molecular patterns reliable to characterize tissue pathology. Technological and methodological improvements have contributed to enhance throughput, sensitivity and spatial resolution. These have stimulated the usage of $\mathrm{MSI}$ in various biomedical applications including oncology before the emergence of direct sampling technologies to assist surgical decisions. This chapter addressed the current challenges of the field including data analysis, molecular identification and reproducibility towards moving MSI closer to clinical routine.

The MSI field in biomedical research has been very dynamic over the past 20 years and produced innovative investigations ranging from instrumental development to application perspectives. This review illustrated the very diverse specialities and accomplishments of the field, which are sometimes challenging to keep track with, but necessary to envision innovative approaches to push the field further. Since its publication, already numerous publications extended the field of MSI illustrating the still on-going effervescence of the research such as new methodologies to improve lipid-imaging identification $(375,376)$ or to reach spatial resolution at the subcellular level with MALDI-MSI (377). In addition, specific tools for real-time tissue classification have been reported employing dedicated hand pieces and ambient ionization sources for surgical applications $(30,31)$. The multitude of capabilities and technologies now available for tissue analysis by mass spectrometry could 
be expected to show distinctive strength towards MSI enrolment in routine diagnostics over the coming years. Previous work has been reported exploring the link between the molecular patterns of REIMS and DESI (304), and complementary investigations in the future to build more precise libraries could be expected.

\section{Reproducibility of rapid evaporative ionization mass spectrometry}

The reliability of the REIMS technology is crucial to move from applied research to routine diagnostics use. In chapter 3 , the reproducibility of REIMS is in focus for the classification of biological tissues in a round robin, multi-site study. Identical equipment and harmonized methodology were employed to analyse locally provided meat samples and breast resection tissues. Normal and tumour breast tissues could be correctly classified across several countries using models based on the data from other countries. In addition, the combination of data from multiple sites improved the classification accuracy for breast tissue recognition.

Further investigations on the reproducibility of REIMS for the classification of biological tissues are warranted. The multicentre study focused on the establishment of the methodology and was limited to a few clinical samples per site $(<10)$ on 3 sites. A broader study including more samples and more sites would be a next step. Future research should separate two main objectives, which are the intrinsic reproducibility of the REIMS technology itself and the reproducibility of REIMS for the application to the classification of clinical tissues.

First, there is a necessity to provide robust results of the REIMS technology itself for classification of biological samples. This involves solely testing the reproducibility of REIMS using samples and a sampling method that present the less possible variability. The tissue samples could be 3 to 5 standard samples that can be produced with high reproducibility. For instance, they could be controls provided by the national institute of standards and technology that provide decent signals for comparison, or cell lines, already proven to be suitable for REIMS analysis in high throughput fashion (378).

The sampling could be performed using a $\mathrm{CO}_{2}$ laser set up that provides better precision (306) than electrocautery. It would lead measurements to be independent from the variability induced by the handling of an operator - even if shown not significant in this study, and from the utilization of diverse heat generators. This would enable to compare results on multiple sites, independently from the affiliations to clinical sites, which should enable the coordination of more partners. Experiments should be performed regularly, repeated and reproduced over months, under the same conditions to focus the investigations purely on the assessment of the REIMS technology itself. This investigation could be put in perspective with the influence of technical parameters, such as the detector gain check, the influence of the pressures, the flowrate of solvent, etc.

Next, the application of REIMS for the classification of clinical tissues needs to focus on the clinical methodology and deal with the potential biological variance of tumour 
heterogeneity. The constitution of a database built on the data acquired on multiple centres would enable better cross-validation results and therefore more sites have to be included in a future study. In addition, each site has to provide a significant amount of samples to cover the heterogeneity of the pathology of the resection tumour entity. The differences in ethnicity of the local population could be expected to lead to distinct tumour molecular patterns. Moreover, cross validation of pathology examination between sites appear also as a nice strategy to align the potential subjective sampling examination and to constitute more accurate databases. Finally, recognition of in vivo signals generated on multiple sites could appear as an important proof of principle to increase the external validity of the technology towards surgical implementation.

\section{Molecular margin of resection and in vivo record of aerosols in the operating room}

Real-time mass spectrometric tissue classifiers were developed to assist surgeons during tumour resection. In chapter 4, REIMS was used to build a library of ex vivo molecular patterns on resected tissues from patients operated for breast tumour surgery. The model was validated with histopathology and classified adipose, stroma and tumour tissues. This model enabled recognition of tissue types in vivo by analysing the electrosurgical vapours during a breast-conserving surgery. Moreover, tumour border stroma and tumour remote stroma were classified with both REIMS and DESI-MS. These results demonstrated the potential of breast stroma towards reaching in real-time knowledgeable information about tumour localisation to perform more precise breast-conserving surgeries.

Better characterisation of the stromal changes surrounding breast tumours is required to evaluate the existence of a molecular margin and to show its detection during surgery. A precise sampling of tumour-tissue margin is needed to establish how far from the tumour stromal changes can be detected. Moreover, this phenomenon could be heterogeneous between tumours, therefore its characterisation for diverse tumour types should be considered.

The hardware of the mobile mass spectrometer needs to be improved to meet the requirements in the operation room. Substantial modifications towards implementation beyond the research purpose should be considered such as reduction of the noise level to avoid disturbance of communication between the operating staff. In addition, the development of portable mass spectrometers (379) could inspire the new designs of such instruments to facilitate their handling and their incorporation in operating rooms.

The next steps for the analysis of electrosurgical vapours in vivo by REIMS need to involve a better methodology to correlate localisation of tissue dissected by the surgeon in time and space, and molecular pattern signal. The employed methodology based on the synchronisation of video records can assure with confidence in vivo tissue sampling and molecular signal, but remains laborious and challenging. Combination of REIMS in vivo measurement with post-resection micro-CT performed on the resection specimen (380) or even the integration of a spatially navigated surgical electrocautery with the real-time lipid 
patterns (381) are promising directions to enable more knowledgeable acquisition of data in the operating room.

The implementation of the in vivo measurements in the operating rooms of Maastricht University Medical Centre+ is expected to be beneficial to other investigations. Beyond breast surgeries, in vivo measurements were also performed during head and neck and sarcoma surgeries employing a diathermic knife. These generated intense and different molecular patterns of potential interest towards extending the utilisation of REIMS for these surgical specialties. In addition, investigations of the combination of a $\mathrm{CO}_{2}$ laser sampling with REIMS should benefit in vivo measurements during tumour resections of the pharynx and the larynx and the development of new investigations such as the prediction of fracture-healing (382).

\section{Lipid patterns to characterise tumour heterogeneity and cell death}

Tumour heterogeneity affects clinical decisions beyond the surgery room. In chapter 5, REIMS was used on resected liver tissues to demonstrate the benefit of real-time lipid patterns for cancer precision medicine. Its use was illustrated on two biological questions. First, primary tumour (hepatocellular carcinoma, HCC) patterns were shown to be more similar to liver parenchyma patterns than secondary tumour (metastatic adenocarcinoma, MAC) patterns. Moreover, tumour heterogeneity appeared greater in HCC than MAC based on lipid patterns. Then, MAC and other tumours were reported to share a common molecular lipid to discriminate necrotic from viable tumours. Closely related sphingolipids, including ceramides, bioactive lipids involved in cell death characterized this pattern. Targeted analysis on these lipids enabled sub classification of tumours based specific metabolic shifts. These results opened new perspectives for tumour characterization based on specific tumour metabolic pathways.

The investigation was performed using a needle to generate electrosurgical vapours with a head generator. As the end-message was not focused on the use of lipid patterns for intraoperative surgical decisions but more towards tailoring diverse clinical interventions to the tumour metabolic phenotype, using a more delicate, less destructive tool could be a more suitable option.

Besides, the biological variance of the lipid patterns, to reflect tumour heterogeneity and to depict metabolic shifts in necrosis need to be better understood. For instance, the study of necrosis could be explored using biological assays with cell lines analyses to characterise the effect of ischemic or hypoxic conditions on the lipid patterns of dead cells.

\section{Eclectic molecular patterns to reflect tissue pathology}

The mass spectrometry modality can offer high confidence for structural characterisation of molecules. Tissue classification investigations can be complemented by experiments for higher level of molecular identification and bring an understanding to the molecular pathobiology underlying histopathological changes between tissue types. Chapters 4 and 5 
illustrate the diversity of the molecular patterns that can reflect tissue classification. Principal component analysis of the lipid pattern libraries enabled to screen mass features discriminative of tissue types to lead for potential biological interpretation of these classifiers.

In chapter 4, the main 3 tissue components of breast cancer resection, which are tumour, stroma and adipose, were classified by lipid patterns mainly characterized by intense glycerophospholipids, fatty acids and triglycerides respectively. The heterogeneity of these lipid classes can be associated with the very heterogeneous histology of healthy and tumour breast tissues. However, no particular biological link can be established between these tissues, being simply classified by diverse lipid patterns, certainly representative their main lipid composition.

In chapter 5, the classification of liver parenchyma, hepatocellular carcinoma and metastatic adenocarcinoma show an alignment along the principal component 1 to screen discriminative mass features. However, the multiple potential species using a resolution of a time-of-flight analyser limited the confidence in the lipid identification. Moreover, the biological links between the diverse glycerophospholipid classes are difficult to establish limiting again any biological meaning. Interestingly, ceramides and related sphingolipids discriminated necrotic from viable metastatic adenocarcinomas and in other human tumours. Metabolic links have been reported between ceramides, acylceramides, lactosylceramides and sphingomyelins and their involvement in the process and the regulation of cell death gave an interesting perspective for biological interpretation.

While the classification of remote tumour stroma and tumour border stroma surrounding breast tumours reported mainly a single mass feature for their discrimination by DESI-MS, assigned as lactate dimer, same comparison by REIMS led to the impossibility to attribute the classification to any specific molecules, as the PCA did not enable screening for discriminative mass features. Mass features with low intensity and therefore not depicted by PCA are probably substantial for the classification obtained after the critical contribution of the supervision by LDA.

Mass spectrometric molecular patterns may range from "a silver bullet" to "uncharacterised molecular pattern", similarly to already routinely used diagnostics biomarkers, such as for breast cancer with one single marker (BRCA1, HER2, etc.) or a combination of biomarkers (MammaPrint). It may depend on the analytical platform and the biological comparison due to the eclectic regulations. Accumulation or deficit of molecules can come in play to picture metabolic phenotypes that can then be detected. Nevertheless, worth mentioning that while molecular patterns have the benefit to provide rapid measurement to assess disease state, they appear by definition less prone to predict the susceptibility of disease developments in comparison to genetic biomarkers. The strength of mass spectrometric molecular patterns may mainly reside on the analysis of present phenotypes for diagnostic and prognostic. 


\section{Samenvatting in het Nederlands}

Oncologische chirurgie is een hoeksteen in de behandeling van patiënten met kanker. Het heeft tot doel al het tumorweefsel te verwijderen om de patiënt van kanker te genezen, terwijl gezonde weefsels worden gespaard om biologische functies te behouden. Intraoperatieve beslissingen bij de uitvoering van een ressectie zijn van cruciaal belang voor het resultaat voor de patiënt (9-11). Dit is deels te wijten aan het feit dat de macroscopische identificatie ter plaatse van tumor of gezond weefsel vaak lastig is voor chirurgen. Deze intraoperatieve analytische uitdaging heeft geleid tot de ontwikkeling van real-time massaspectrometrische methoden en technologieën zoals snelle REIMS-analyse (Rapid Electrospray lonization Mass Spectrometry) van elektrochirurgische dampen, om weefsels in de operatiekamer te classificeren en daarom histopathologische beoordeling te bieden ter ondersteuning van efficiënte chirurgische beslissingen op basis van moleculaire patronen. Het werk beschreven in dit proefschrift ondersteunt de visie dat de REIMStechnologie kan worden geïmplementeerd in chirurgische omgevingen over de hele wereld voor routinematige, intraoperatieve beoordeling van de tumor marge tijdens chirurgische resectie van een tumor.

Dit proefschrift bespreekt de stand van de techniek van het onderzoeksveld van weefselclassificatie op basis van massaspectrometrische patronen. Daarin zijn de reproduceerbaarheid van de REIMS-technologie, de betrouwbaarheid ervan voor diagnostisch gebruik en de toepassingen ervan ter ondersteuning van chirurgische resectie essentiële elementen. In dit proces speelt tumor heterogeniteit een cruciale rol. We gaan in op de respectieve implicaties, stellen onderzoeken voor om deze lijn van werk voort te zetten en uit te breiden, en bespreken de lipidenpatronen, de basis van deze classificatie die de onderliggende weefsel-pathobiologie weerspiegelen.

\section{Beeldvormende Massaspectrometrie, bijdragen en onderzoeksrichtingen}

In hoofdstuk 1 hebben we de evolutie van beeldvormende massaspectrometrie (MSI) besproken die hebben geleid tot het visualiseren van moleculaire patronen die weefselpathologie betrouwbaar karakteriseren. Technologische en methodologische verbeteringen hebben bijgedragen tot een verbeterde doorvoer, gevoeligheid en ruimtelijke resolutie. Dit heeft het gebruik van MSI in verschillende biomedische toepassingen, waaronder oncologie, gestimuleerd vóór de opkomst van directe bemonsteringstechnologieën om beter gefundeerde chirurgische beslissingen te faciliteren. We hebben de huidige uitdagingen in het veld onderzocht en verder ontwikkeld, waaronder gegevensanalyse, moleculaire identificatie en reproduceerbaarheid, om de klinische translatie van MSI makkelijker te maken.

Het MSI-veld in biomedisch onderzoek is zeer dynamisch en heeft innovatieve studies opgeleverd, variërend van instrumentele ontwikkeling tot toepassingsperspectieven. Ons overzicht illustreerde de zeer diverse specialiteiten, disciplines en doorbraken in het veld, die noodzakelijk zijn om innovatieve benaderingen te implementeren, die op hun beurt dit onderzoeksveld versneld voortduwen. Sinds de publicatie van hoofdstuk 2 hebben al talrijke 
publicaties het gebied van MSI uitgebreid, wat de onderzoeksdynamiek illustreert. Nieuwe methodes om de identificatie van lipidenbeeldvorming te verbeteren $(375,376)$ of om een ruimtelijke resolutie op subcellulair niveau te bereiken met MALDI-MSI (377) zijn daar concrete voorbeelden van. Bovendien zijn er specifieke tools voor real-time weefselclassificatie gerapporteerd die speciale chirurgische gereedschappen en omgevingsionisatiebronnen gebruiken voor chirurgische toepassingen $(30,31)$. De veelheid aan mogelijkheden en technologieën die nu beschikbaar zijn voor weefselanalyse door middel van massaspectrometrie, zal naar verwachting de komende jaren het onderscheidende vermogen van MSI in routinematige diagnostiek verder groeien. Eerder werk is gerapporteerd waarin het verband tussen de moleculaire patronen van REIMS en DESI (304) is onderzocht en we kunnen in de toekomst meer complementair onderzoek verwachten om nauwkeurigere bibliotheken te bouwen.

\section{Reproduceerbaarheid van snelle verdampingsionisatie-massaspectrometrie (REIMS)}

De betrouwbaarheid van de REIMS-technologie is cruciaal om te bewijzen dat toegepast onderzoek ingezet kan worden voor diagnostisch gebruik. In hoofdstuk 3 hebben we de reproduceerbaarheid van REIMS bestudeerd voor de classificatie van biologische weefsels in een round-robin, multi-center studie. Identieke apparatuur en methodologie werd gebruikt om lokaal verstrekte dierlijke monsters en weefsels van borstresectie te analyseren. Normaal borstweefsel en tumorweefsel kunnen in meerdere landen correct worden geclassificeerd met behulp van modellen die zijn gebaseerd op gegevens uit andere landen. Bovendien verbeterde de combinatie van gegevens van meerdere locaties de classificatienauwkeurigheid voor de herkenning van borstweefsel.

Verder onderzoek naar de reproduceerbaarheid van REIMS voor de classificatie van biologische weefsels is nodig. Onze multi-center studie was gericht op het vaststellen van de methodologie en was beperkt tot enkele klinische monsters per site $(<10)$ op slechts 3 sites. Een bredere studie met meer monsters en meer sites zou een volgende stap naar validatie zijn. Toekomstig onderzoek zou twee hoofddoelstellingen moeten scheiden, namelijk de intrinsieke reproduceerbaarheid van de REIMS-technologie zelf en de reproduceerbaarheid van REIMS voor de toepassing op de classificatie van klinische weefsels.

Hiervoor is het ten eerste nodig om robuuste resultaten van de REIMS-technologie zelf te realiseren voor de classificatie van biologische monsters. Dit houdt in dat alleen de reproduceerbaarheid van REIMS wordt getest met behulp van steekproeven middels een steekproefmethode die zo min mogelijk variabiliteit vertoont. De weefselmonsters kunnen 3 tot 5 standaardmonsters zijn die kunnen worden geproduceerd met een hoge reproduceerbaarheid, zoals de controles kunnen worden geleverd door een nationale instituut voor normen en technologie met fatsoenlijke signalen die een goed vergelijking mogelijk maken. Cellijnen waarvan al is bewezen dat ze geschikt zijn voor REIMS-analyse met een korte doorlooptijd (378) zijn daarvoor ook zeer geschikt. De bemonstering kan worden uitgevoerd met behulp van een CO2-laseropstelling die een betere precisie (306) 
biedt dan elektro-cauterisatie. Hiermee wordt een studie ook onafhankelijk van de operator variabiliteit - zelfs als deze niet significant is gebleken in ons onderzoek. Dit zou het mogelijk maken om resultaten op meerdere sites te vergelijken, onafhankelijk van de klinische sites, wat de inclusie van meer partners mogelijk zou moeten maken. Experimenten moeten regelmatig worden uitgevoerd, herhaald en gedurende maanden worden gereproduceerd, onder dezelfde omstandigheden, om het onderzoek uitsluitend te richten op de beoordeling en kwaliteitscontrole van de REIMS-technologie zelf. Hierbij kan de invloed van technische parameters zoals de detectorversterkingscontrole, de invloed van de drukken, de stroomsnelheid van oplosmiddel, enz eenvoudig meegenomen worden.

Vervolgens moet de toepassing van REIMS voor de classificatie van klinische weefsels zich concentreren op de klinische methodologie en de mogelijke biologische variantie gerelateerd van tumorheterogeniteit in kaart brengen. De samenstelling van een database die is gebaseerd op de gegevens die over meerdere centra zijn verzameld, zou betere kruisvalidatie mogelijk maken. Juist daarom moeten meer sites worden opgenomen in een toekomstige studie. Bovendien moet elke site een aanzienlijk aantal monsters leveren om de heterogeniteit van de pathologie van de het resectie materiaal goed in kaart te brengen. De verschillen in etniciteit van de lokale bevolking zullen naar verwachting leiden tot verschillende moleculaire metabole patronen van tumoren. Bovendien lijkt kruisvalidatie van pathologieonderzoek tussen locaties ook een mooie strategie om het potentiële subjectieve steekproefonderzoek op elkaar af te stemmen en om nauwkeurigere, internationale databases te realiseren. Ten slotte zou herkenning van in-vivo signalen, die op meerdere sites worden gegenereerd, chirurgische implementatie van de technologie en de externe validatie ervan vergroten.

\section{Moleculaire resectie marge en in vivo opname van aerosolen in de operatiekamer}

Realtime massaspectrometrische weefselclassificatoren werden ontwikkeld om chirurgen te assisteren tijdens tumorresecties. In hoofdstuk 4 hebben we REIMS gebruikt om een bibliotheek op te bouwen van ex vivo moleculaire patronen van weggesneden weefsels van patiënten die geopereerd zijn voor borstkanker. We valideerden het model met histopathologie en classificeerden adipeus, stroma en tumorweefsel. Dit model maakte herkenning van verschillende typen weefsels in vivo- mogelijk door de analyse van elektrochirurgische dampen tijdens een borstsparende operatie. Bovendien hebben we tumorgrensende stroma en tumorafgelegen stroma geclassificeerd met REIMS en DESI-MS. Deze resultaten demonstreren het potentieel van borststroma om in realtime kennis over tumorlokalisatie te verkrijgen en zo nauwkeurigere borstsparende operaties uit te kunnen voeren.

Een betere karakterisering van de stromale veranderingen rond borsttumoren is vereist om de moleculaire marge rond de tumor te evalueren en deze tijdens een operatie preciezer aan te tonen. Een nauwkeurige bemonstering van de tumorweefselmarge laat zien hoe ver van de tumor stromale veranderingen kunnen worden gedetecteerd. Dit fenomeen kan 
tussen tumoren verschillen, daarom moet de karakterisering ervan worden uitgebreid met verschillende tumortypen.

De hardware van de mobiele massaspectrometer moet worden verbeterd om aan de eisen in de operatiekamer te voldoen. Aanzienlijke wijzigingen in de richting van implementatie, die verder gaan dan het onderzoeksdoel beschreven in dit proefschrift, zijn noodzakelijk. Zo moet het geluidsniveau worden verminderd om de communicatie tussen het personeel in de OK niet te verstoren. Bovendien zou de ontwikkeling van draagbare massaspectrometers (379) een nieuwe ontwerp van dergelijke instrumenten mogelijk maken die het gebruik van op MS gebaseerde intra-operatieve diagnostiek in operatiekamers vergemakkelijken.

De volgende stappen voor de analyse van elektrochirurgische dampen in vivo door REIMS moeten zich richten op een betere methodologie om de lokalisatie van door de chirurg verwijderd weefsel ruimtelijk en tijdopgelost te correleren met de beschikbare patiënt informatie (CT, MRI, operatie gebied). Dit maakt het mogelijk om het lipidenpatroonsignaal preciezer te acquireren in de operatiekamer. De combinatie in-vivo REIMS metingen met post-resectie micro-CT (380) of zelfs de integratie van een ruimtelijk genavigeerde chirurgische elektrocauterisatie met de real-time lipidenpatronen (381) zijn veelbelovende onderzoeksrichtingen voor de toekomst.

De uitvoering van de in-vivo metingen in de operatiekamers van het Maastricht Universitair Medisch Centrum zijn verwachting gunstig voor ander toepassingsgericht onderzoek. Naast borstoperaties hebben we ook in vivo metingen uitgevoerd tijdens hoofd/nek tumorresecties alsmede sarcoomoperaties met behulp van een diathermisch mes. Deze resultaten lieten intense en verschillende moleculaire patronen die van belang zijn voor het gebruik van REIMS in deze chirurgische specialiteiten. Onderzoek naar de combinatie van $\mathrm{CO}_{2}$-lasersampling met REIMS tijdens in-vivo metingen bij tumorresecties van de keelholte en het strottenhoofd laten veelbelovende resultaten zien. De ontwikkeling van nieuwe toepassingen, zoals de voorspelling van fractuurgenezing (382), laten zien dat dit veld nog volop in ontwikkeling is.

\section{Lipidenpatronen om tumorheterogeniteit en celdood te karakteriseren}

Tumor-heterogeniteit beïnvloedt klinische beslissingen buiten de operatiekamer. In hoofdstuk 5 hebben we REIMS op weggesneden leverweefsel toegepast gebaseerd op realtime lipidenpatronen ten behoeve van precisiegeneeskunde in leveroncologie. We hebben deze techniek toegepast op twee biomedische studies. Ten eerste hebben we aangetoond dat patronen van primaire tumoren (hepatocellulair carcinoom, HCC) een grotere gelijkenis vertonen met patronen van leverparenchym dan patronen van secundaire tumoren (gemetastaseerd adenocarcinoom, MAC). De heterogeniteit van de tumor lijkt groter in HCC dan in MAC. Ten tweede werd vastgesteld dat MAC en andere tumoren een gemeenschappelijk lipidenpatroon delen waarmee necrotisch weefsel onderscheiden kan worden van levensvatbare tumoren. Een groep sphingolipiden, waaronder ceramides, bioactieve lipiden die betrokken zijn bij celdood, kenmerken dit patroon. Een gerichte 
analyse van deze lipiden maakt een subclassificatie van tumoren mogelijk op basis van specifieke metabole verschuivingen. Dir resulteerde in nieuwe perspectieven voor tumorkarakterisering op basis van tumor metabolisme.

Een fundamentele studie naar het gebruik van lipidenpatronen voor intraoperatieve chirurgische diagnostiek vereist het gebruik van een delicater, minder destructief hulpmiddel. Om die reden is een elektrochirurgische naald. gebruikt om een beter inzicht te verschaffen in de REIMS techniek. Daarmee is een beter inzicht verkregen in de biologische variantie van de lipidenpatronen gerelateerd aan de HCC en MAC tumorheterogeniteit. De metabole verschuivingen van ceramiden in necrotisch weefsel zijn daarin meegenomen. De studie van necrose zou verder onderzocht kunnen worden met behulp van biologische assays die gebruik maken van cellijnen om het effect van ischemische of hypoxische aandoeningen op de lipidenpatronen van dode cellen te karakteriseren.

\section{Eclectische moleculaire patronen in weefselpathologie}

Massaspectrometrie biedt ongekende mogelijkheden voor voor structurele karakterisering van moleculen. Weefselclassificatie is aangevuld met experimenten voor betrouwbare moleculaire identificatie en heeft inzicht gegeven in de moleculaire pathobiologie die ten grondslag ligt aan histopathologische veranderingen tussen weefseltypen. Hoofdstukken 4 en 5 illustreren de diversiteit van de moleculaire patronen die voor weefselclassificatie worden gebruikt. Principale componenten analyse van de lipidenpatronen maakte het mogelijk om functies te screenen die onderscheid maken tussen weefseltypen en deze te koppelen aan biologische interpretatie van deze classificatoren.

In hoofdstuk 4 werden de drie belangrijkste weefselcomponenten van de resectie van borstkanker, namelijk tumor, stroma en vetweefsel, geclassificeerd op basis van lipidenpatronen die voornamelijk worden gekenmerkt door respectievelijk intense glycerofosfolipiden, vetzuren en triglyceriden. De heterogeniteit van deze lipidenklassen is geassocieerd met de histologie van zowel tumorweefsel en van gezond borstweefsel. Er kan echter geen specifiek biologisch verband worden gelegd tussen deze weefsels.

In hoofdstuk 5 laat de classificatie van leverparenchym, hepatocellulair carcinoom en gemetastaseerd adenocarcinoom een verdeling zien langs de hoofdcomponent 1 . De beperkte resolutie van een time-of-flight-analysator, beperkte de mogelijkheden voor lipidenidentificatie. Bovendien zijn de biologische verbanden tussen de diverse glycerofosfolipidenklassen moeilijk vast te stellen, wat het trekken van biologische conclusies bemoeilijkt. Wel vonden we dat ceramiden en verwante sfingolipiden necrotisch weefsel onderscheiden van levensvatbare metastatische adenocarcinomen en andere menselijke tumoren. Er zijn metabolische verbanden gerapporteerd tussen ceramiden, acylceramiden, lactosylceramiden en sfingomyelines en hun betrokkenheid bij de regulering van celdood. 
De classificatie van afgelegen tumorstroma en tumorgrensstroma rond borsttumoren voornamelijk door een enkel DESI-MS moleculaire piek gediscrimineerd. Dit is geïdentificeerd als een lactaatdimeer. PCA screening op discriminerende massaeigenschappen bleek niet mogelijk te zijn. Massa-kenmerken met lage intensiteit zijn waarschijnlijk substantieel voor de classificatie die is verkregen na gesuperviseerde LDA.

Net als bij reeds routinematige diagnostische biomarkers zoals voor borstkanker met één enkele marker (BRCA1, HER2, enz.) of een combinatie van biomarkers (MammaPrint), kunnen massaspectrometrische moleculaire patronen variëren van 'een wondermiddel' tot 'niet-gekarakteriseerd moleculair patroon'. Het succes van de methode hangt af van het analytische platform, de biologische vergelijking, moleculair accumulatie of consumptie die allen een rol spelen bij het in beeld brengen van metabole fenotypes. Dit proefschrift laat zien dat lipidenpatronen het voordeel hebben dat ze een snelle meting bieden om de ziektetoestand te beoordelen, maar dat ze minder gevoeligheid hebben om ziekteontwikkelingen te voorspellen in vergelijking met genetische biomarkers. De kracht van massaspectrometrische moleculaire patronen ligt voornamelijk in de analyse van de moleculaire fenotypes voor diagnostiek en prognostiek. 
Chapter 8

Appendix 


\section{References}

1. Mattiuzzi C, Lippi G. Current Cancer Epidemiology. Journal of epidemiology and global health 2019;9:217-22

2. Peters JM, Gonzalez FJ. The Evolution of Carcinogenesis. Toxicological sciences : an official journal of the Society of Toxicology 2018;165:272-6

3. Puisieux A, Pommier RM, Morel AP, Lavial F. Cellular Pliancy and the Multistep Process of Tumorigenesis. Cancer cell 2018;33:164-72

4. Hanahan D, Weinberg Robert A. Hallmarks of Cancer: The Next Generation. Cell 2011;144:646-74

5. Pauli C, Hopkins BD, Prandi D, Shaw R, Fedrizzi T, Sboner A, et al. Personalized In Vitro and In Vivo Cancer Models to Guide Precision Medicine. Cancer discovery 2017;7:462-77

6. Hanahan D, Weinberg RA. The hallmarks of cancer. Cell 2000;100:57-70

7. Burrell RA, McGranahan N, Bartek J, Swanton C. The causes and consequences of genetic heterogeneity in cancer evolution. Nature 2013;501:338-45

8. Tabassum DP, Polyak K. Tumorigenesis: it takes a village. Nature reviews Cancer 2015;15:473-83

9. Zhao J, Sawo P, Rensen SS, Rouflart MMJ, Winstanley A, Vreuls CPH, et al. Impact of chemotherapy-associated liver injury on tumour regression grade and survival in patients with colorectal liver metastases. HPB : the official journal of the International Hepato Pancreato Biliary Association 2018;20:147-54

10. Margonis GA, Sergentanis TN, Ntanasis-Stathopoulos I, Andreatos N, Tzanninis IG, Sasaki K, et al. Impact of Surgical Margin Width on Recurrence and Overall Survival Following RO Hepatic Resection of Colorectal Metastases: A Systematic Review and Meta-analysis. Annals of surgery 2018;267:1047-55

11. Tummers WS, Groen JV, Sibinga Mulder BG, Farina-Sarasqueta A, Morreau J, Putter $\mathrm{H}$, et al. Impact of resection margin status on recurrence and survival in pancreatic cancer surgery. The British journal of surgery 2019;106:1055-65

12. Gray RJ, Pockaj BA, Garvey E, Blair S. Intraoperative Margin Management in BreastConserving Surgery: A Systematic Review of the Literature. Annals of Surgical Oncology 2018;25:18-27

13. Bae MS, Bernard-Davila B, Sung JS, Morris EA. Preoperative breast MRI features associated with positive or close margins in breast-conserving surgery. European journal of radiology 2019;117:171-7

14. Kittaneh M, Montero AJ, Glück S. Molecular profiling for breast cancer: a comprehensive review. Biomark Cancer 2013;5:61-70

15. Alkabban FM, Ferguson T. Cancer, Breast. StatPearls. Treasure Island (FL): StatPearls Publishing; 2020.

16. Alam IS, Steinberg I, Vermesh O, van den Berg NS, Rosenthal EL, van Dam GM, et al. Emerging Intraoperative Imaging Modalities to Improve Surgical Precision. Molecular imaging and biology : MIB : the official publication of the Academy of Molecular Imaging 2018

17. Stammes MA, Bugby SL, Porta T, Pierzchalski K, Devling T, Otto C, et al. Modalities for image- and molecular-guided cancer surgery. The British journal of surgery 2018;105:e69-e83 
18. Davarzani N, Hutchins GGA, West NP, Hewitt LC, Nankivell M, Cunningham D, et al. Prognostic value of pathological lymph node status and primary tumour regression grading following neoadjuvant chemotherapy - results from the MRC OEO2 oesophageal cancer trial. Histopathology 2018;72:1180-8

19. Pena GP, Andrade-Filho Jde S. How does a pathologist make a diagnosis? Archives of pathology \& laboratory medicine 2009;133:124-32

20. Foucar E. Diagnostic decision-making in anatomic pathology. American journal of clinical pathology 2001;116 Suppl:S21-33

21. Svajdler M, Svajdler P. Frozen section: history, indications, contraindications and quality assurance. Ceskoslovenska patologie;54:58-62

22. Lee DY, Kang SH, Kim JH, Kim MS, Oh KH, Woo JS, et al. Survival and recurrence of resectable tongue cancer: Resection margin cutoff value by T classification. Head \& neck 2018;40:283-91

23. Lossl $\mathrm{P}$, van de Waterbeemd $\mathrm{M}$, Heck AJ. The diverse and expanding role of mass spectrometry in structural and molecular biology. The EMBO journal 2016;35:2634-57

24. Zhang YV, Wei B, Zhu Y, Zhang Y, Bluth MH. Liquid Chromatography-Tandem Mass Spectrometry: An Emerging Technology in the Toxicology Laboratory. Clinics in laboratory medicine 2016;36:635-61

25. Caprioli RM, Farmer TB, Gile J. Molecular imaging of biological samples: localization of peptides and proteins using MALDI-TOF MS. Anal Chem 1997;69:4751-60

26. Yanagisawa K, Shyr Y, Xu BJ, Massion PP, Larsen PH, White BC, et al. Proteomic patterns of tumour subsets in non-small-cell lung cancer. Lancet (London, England) 2003;362:433-9

27. Vaysse PM, Heeren RMA, Porta T, Balluff B. Mass spectrometry imaging for clinical research - latest developments, applications, and current limitations. The Analyst 2017;142:2690-712

28. Takats Z, Wiseman JM, Gologan B, Cooks RG. Mass spectrometry sampling under ambient conditions with desorption electrospray ionization. Science 2004;306:471-3

29. Balog J, Sasi-Szabó L, Kinross J, Lewis MR, Muirhead LJ, Veselkov K, et al. Intraoperative tissue identification using rapid evaporative ionization mass spectrometry. Science Translational Medicine 2013;5:194ra93

30. Zhang J, Rector J, Lin JQ, Young JH, Sans M, Katta N, et al. Nondestructive tissue analysis for ex vivo and in vivo cancer diagnosis using a handheld mass spectrometry system. Sci Transl Med 2017;9

31. Saudemont P, Quanico J, Robin YM, Baud A, Balog J, Fatou B, et al. Real-Time Molecular Diagnosis of Tumors Using Water-Assisted Laser Desorption/Ionization Mass Spectrometry Technology. Cancer cell 2018;34:840-51.e4

32. Cho JY, Sung HJ. Proteomic approaches in lung cancer biomarker development. Expert review of proteomics 2009;6:27-42

33. Mallick P, Kuster B. Proteomics: a pragmatic perspective. Nature biotechnology 2010;28:695-709

34. Xue YJ, Gao H, Ji QC, Lam Z, Fang X, Lin ZJ, et al. Bioanalysis of drug in tissue: current status and challenges. Bioanalysis 2012;4:2637-53 
35. Suhre K. Metabolic profiling in diabetes. The Journal of endocrinology 2014;221:R75-85

36. Espina V, Wulfkuhle JD, Calvert VS, VanMeter A, Zhou W, Coukos G, et al. Lasercapture microdissection. Nature protocols 2006;1:586-603

37. Gauthier DJ, Sobota JA, Ferraro F, Mains RE, Lazure C. Flow cytometry-assisted purification and proteomic analysis of the corticotropes dense-core secretory granules. Proteomics 2008;8:3848-61

38. Walch A, Rauser S, Deininger SO, Hofler H. MALDI imaging mass spectrometry for direct tissue analysis: a new frontier for molecular histology. Histochemistry and cell biology 2008;130:421-34

39. Rubakhin SS, Jurchen JC, Monroe EB, Sweedler JV. Imaging mass spectrometry: fundamentals and applications to drug discovery. Drug discovery today 2005; 10:823-37

40. Schwamborn K. The Importance of Histology and Pathology in Mass Spectrometry Imaging. Advances in cancer research 2017;134:1-26

41. Rauser S, Deininger SO, Suckau D, Hofler H, Walch A. Approaching MALDI molecular imaging for clinical proteomic research: current state and fields of application. Expert review of proteomics 2010;7:927-41

42. Schwamborn K, Kriegsmann M, Weichert W. MALDI imaging mass spectrometry From bench to bedside. Biochimica et biophysica acta 2016

43. Wu C, Dill AL, Eberlin LS, Cooks RG, Ifa DR. Mass spectrometry imaging under ambient conditions. Mass spectrometry reviews 2013;32:218-43

44. Casadonte R, Caprioli RM. Proteomic analysis of formalin-fixed paraffin-embedded tissue by MALDI imaging mass spectrometry. Nature protocols 2011;6:1695-709

45. Ly A, Buck A, Balluff B, Sun N, Gorzolka K, Feuchtinger A, et al. High-mass-resolution MALDI mass spectrometry imaging of metabolites from formalin-fixed paraffinembedded tissue. Nature protocols 2016;11:1428-43

46. Heeren RMA. Getting the picture: The coming of age of imaging MS. International journal of mass spectrometry 2015;377:672-80

47. Sodhi RN. Time-of-flight secondary ion mass spectrometry (TOF-SIMS):--versatility in chemical and imaging surface analysis. The Analyst 2004;129:483-7

48. Chaurand P, Schwartz SA, Billheimer D, Xu BJ, Crecelius A, Caprioli RM. Integrating histology and imaging mass spectrometry. Anal Chem 2004;76:1145-55

49. Todd PJ, Schaaff TG, Chaurand P, Caprioli RM. Organic ion imaging of biological tissue with secondary ion mass spectrometry and matrix-assisted laser desorption/ionization. Journal of mass spectrometry : JMS 2001;36:355-69

50. Gustafsson JO, Oehler MK, Ruszkiewicz A, McColl SR, Hoffmann P. MALDI Imaging Mass Spectrometry (MALDI-IMS)-application of spatial proteomics for ovarian cancer classification and diagnosis. International journal of molecular sciences 2011;12:773-94

51. Hankin JA, Barkley RM, Murphy RC. Sublimation as a method of matrix application for mass spectrometric imaging. Journal of the American Society for Mass Spectrometry 2007;18:1646-52

52. Addie RD, Balluff B, Bovee JV, Morreau H, McDonnell LA. Current State and Future Challenges of Mass Spectrometry Imaging for Clinical Research. Analytical chemistry 2015;87:6426-33 
53. Baker TC, Han J, Borchers CH. Recent advancements in matrix-assisted laser desorption/ionization mass spectrometry imaging. Current opinion in biotechnology 2016;43:62-9

54. Yang J, Caprioli RM. Matrix sublimation/recrystallization for imaging proteins by mass spectrometry at high spatial resolution. Analytical chemistry 2011;83:572834

55. Cillero-Pastor B, Heeren RM. Matrix-assisted laser desorption ionization mass spectrometry imaging for peptide and protein analyses: a critical review of ontissue digestion. Journal of proteome research 2014;13:325-35

56. Esteve C, Tolner EA, Shyti R, van den Maagdenberg AM, McDonnell LA. Mass spectrometry imaging of amino neurotransmitters: a comparison of derivatization methods and application in mouse brain tissue. Metabolomics : Official journal of the Metabolomic Society 2016;12:30

57. Lanekoff I, Stevens SL, Stenzel-Poore MP, Laskin J. Matrix effects in biological mass spectrometry imaging: identification and compensation. The Analyst 2014;139:3528-32

58. Deininger SO, Cornett DS, Paape R, Becker M, Pineau C, Rauser S, et al. Normalization in MALDI-TOF imaging datasets of proteins: practical considerations. Analytical and bioanalytical chemistry 2011;401:167-81

59. Takats Z, Wiseman JM, Gologan B, Cooks RG. Electrosonic spray ionization. A gentle technique for generating folded proteins and protein complexes in the gas phase and for studying ion-molecule reactions at atmospheric pressure. Analytical chemistry 2004;76:4050-8

60. Porta T, Lesur A, Varesio E, Hopfgartner G. Quantification in MALDI-MS imaging: what can we learn from MALDI-selected reaction monitoring and what can we expect for imaging? Analytical and bioanalytical chemistry 2015;407:2177-87

61. Spraggins JM, Caprioli RM. High-speed MALDI-TOF imaging mass spectrometry: rapid ion image acquisition and considerations for next generation instrumentation. Journal of the American Society for Mass Spectrometry 2011;22:1022-31

62. Wiseman JM, Ifa DR, Zhu Y, Kissinger CB, Manicke NE, Kissinger PT, et al. Desorption electrospray ionization mass spectrometry: Imaging drugs and metabolites in tissues. Proceedings of the National Academy of Sciences of the United States of America 2008;105:18120-5

63. Fernandes AM, Vendramini PH, Galaverna R, Schwab NV, Alberici LC, Augusti R, et al. Direct Visualization of Neurotransmitters in Rat Brain Slices by Desorption Electrospray Ionization Mass Spectrometry Imaging (DESI - MS). Journal of the American Society for Mass Spectrometry 2016;27:1944-51

64. Zhang J, Yu W, Ryu SW, Lin J, Buentello G, Tibshirani R, et al. Cardiolipins Are Biomarkers of Mitochondria-Rich Thyroid Oncocytic Tumors. Cancer research 2016

65. Lostun D, Perez CJ, Licence P, Barrett DA, Ifa DR. Reactive DESI-MS imaging of biological tissues with dicationic ion-pairing compounds. Analytical chemistry 2015;87:3286-93

66. Ifa DR, Eberlin LS. Ambient Ionization Mass Spectrometry for Cancer Diagnosis and Surgical Margin Evaluation. Clinical chemistry 2016;62:111-23 
67. Laskin J, Heath BS, Roach PJ, Cazares L, Semmes OJ. Tissue imaging using nanospray desorption electrospray ionization mass spectrometry. Analytical chemistry 2012;84:141-8

68. Hsu CC, Chou PT, Zare RN. Imaging of Proteins in Tissue Samples Using Nanospray Desorption Electrospray Ionization Mass Spectrometry. Analytical chemistry 2015;87:11171-5

69. Sarsby J, Martin NJ, Lalor PF, Bunch J, Cooper HJ. Top-down and bottom-up identification of proteins by liquid extraction surface analysis mass spectrometry of healthy and diseased human liver tissue. Journal of the American Society for Mass Spectrometry 2014;25:1953-61

70. Wisztorski M, Desmons A, Quanico J, Fatou B, Gimeno JP, Franck J, et al. Spatiallyresolved protein surface microsampling from tissue sections using liquid extraction surface analysis. Proteomics 2016;16:1622-32

71. Nemes P, Vertes A. Laser ablation electrospray ionization for atmospheric pressure, in vivo, and imaging mass spectrometry. Analytical chemistry 2007;79:8098-106

72. Sussulini A, Becker JS, Becker JS. Laser ablation ICP-MS: Application in biomedical research. Mass spectrometry reviews 2017;36:47-57

73. Yanes O, Woo HK, Northen TR, Oppenheimer SR, Shriver L, Apon J, et al. Nanostructure initiator mass spectrometry: tissue imaging and direct biofluid analysis. Analytical chemistry 2009;81:2969-75

74. Prentice BM, Caprioli RM. The Need for Speed in Matrix-Assisted Laser Desorption/Ionization Imaging Mass Spectrometry. Postdoc journal : a journal of postdoctoral research and postdoctoral affairs 2016;4:3-13

75. Ogrinc Potocnik N, Porta T, Becker M, Heeren RM, Ellis SR. Use of advantageous, volatile matrices enabled by next-generation high-speed matrix-assisted laser desorption/ionization time-of-flight imaging employing a scanning laser beam. Rapid communications in mass spectrometry : RCM 2015;29:2195-203

76. Spraggins JM, Rizzo DG, Moore JL, Noto MJ, Skaar EP, Caprioli RM. Next-generation technologies for spatial proteomics: Integrating ultra-high speed MALDI-TOF and high mass resolution MALDI FTICR imaging mass spectrometry for protein analysis. Proteomics 2016;16:1678-89

77. Steven RT, Dexter A, Bunch J. Investigating MALDI MSI parameters (Part 1) - A systematic survey of the effects of repetition rates up to $20 \mathrm{kHz}$ in continuous raster mode. Methods 2016;104:101-10

78. Prentice BM, Chumbley CW, Caprioli RM. High-speed MALDI MS/MS imaging mass spectrometry using continuous raster sampling. Journal of mass spectrometry : JMS 2015;50:703-10

79. Steurer S, Borkowski C, Odinga S, Buchholz M, Koop C, Huland H, et al. MALDI mass spectrometric imaging based identification of clinically relevant signals in prostate cancer using large-scale tissue microarrays. International journal of cancer 2013;133:920-8

80. Jones EA, Towers M, Shrestha B, Hart P, Chapman R, Claude E. 2016; San Antonio.

81. Junttila MR, de Sauvage FJ. Influence of tumour micro-environment heterogeneity on therapeutic response. Nature 2013;501:346-54 
82. Lanni EJ, Rubakhin SS, Sweedler JV. Mass spectrometry imaging and profiling of single cells. Journal of proteomics 2012;75:5036-51

83. Dekker TJ, Balluff BD, Jones EA, Schone CD, Schmitt M, Aubele M, et al. Multicenter matrix-assisted laser desorption/ionization mass spectrometry imaging (MALDI $\mathrm{MSI}$ ) identifies proteomic differences in breast-cancer-associated stroma. Journal of proteome research 2014;13:4730-8

84. Holzlechner M, Strasser K, Zareva E, Steinhauser L, Birnleitner H, Beer A, et al. In Situ Characterization of Tissue-Resident Immune Cells by MALDI Mass Spectrometry Imaging. Journal of proteome research 2017;16:65-76

85. Carlred L, Vukojevic V, Johansson B, Schalling M, Hook F, Sjovall P. Imaging of amyloid-beta in Alzheimer's disease transgenic mouse brains with ToF-SIMS using immunoliposomes. Biointerphases 2016;11:02A312

86. Altelaar AF, Luxembourg SL, McDonnell LA, Piersma SR, Heeren RM. Imaging mass spectrometry at cellular length scales. Nature protocols 2007;2:1185-96

87. Weaver EM, Hummon AB. Imaging mass spectrometry: from tissue sections to cell cultures. Advanced drug delivery reviews 2013;65:1039-55

88. Brison J, Benoit DS, Muramoto S, Robinson M, Stayton PS, Castner DG. ToF-SIMS imaging and depth profiling of HeLa cells treated with bromodeoxyuridine. Surface and interface analysis : SIA 2011;43:354-7

89. Feenstra AD, Duenas ME, Lee YJ. Five Micron High Resolution MALDI Mass Spectrometry Imaging with Simple, Interchangeable, Multi-Resolution Optical System. Journal of the American Society for Mass Spectrometry 2017;28:434-42

90. Spengler B, Hubert M. Scanning microprobe matrix-assisted laser desorption ionization (SMALDI) mass spectrometry: instrumentation for sub-micrometer resolved LDI and MALDI surface analysis. Journal of the American Society for Mass Spectrometry 2002;13:735-48

91. Kompauer $M$, Heiles $S$, Spengler B. Atmospheric pressure MALDI mass spectrometry imaging of tissues and cells at 1.4-mum lateral resolution. Nature methods 2017;14:90-6

92. McDonnell LA, Heeren RM. Imaging mass spectrometry. Mass spectrometry reviews 2007;26:606-43

93. Thiery-Lavenant G, Zavalin Al, Caprioli RM. Targeted multiplex imaging mass spectrometry in transmission geometry for subcellular spatial resolution. Journal of the American Society for Mass Spectrometry 2013;24:609-14

94. Bodenmiller B. Multiplexed Epitope-Based Tissue Imaging for Discovery and Healthcare Applications. Cell systems 2016;2:225-38

95. Giesen C, Wang HA, Schapiro D, Zivanovic N, Jacobs A, Hattendorf B, et al. Highly multiplexed imaging of tumor tissues with subcellular resolution by mass cytometry. Nature methods 2014;11:417-22

96. Enthaler B, Bussmann T, Pruns JK, Rapp C, Fischer M, Vietzke JP. Influence of various on-tissue washing procedures on the entire protein quantity and the quality of matrix-assisted laser desorption/ionization spectra. Rapid communications in mass spectrometry : RCM 2013;27:878-84

97. Lemaire R, Wisztorski M, Desmons A, Tabet JC, Day R, Salzet M, et al. MALDI-MS direct tissue analysis of proteins: Improving signal sensitivity using organic treatments. Analytical chemistry 2006;78:7145-53 
98. Martin-Lorenzo M, Balluff B, Sanz-Maroto A, van Zeijl RJ, Vivanco F, Alvarez-Llamas $G$, et al. 30mum spatial resolution protein MALDI MSI: In-depth comparison of five sample preparation protocols applied to human healthy and atherosclerotic arteries. Journal of proteomics 2014;108:465-8

99. Seeley EH, Oppenheimer SR, Mi D, Chaurand P, Caprioli RM. Enhancement of protein sensitivity for MALDI imaging mass spectrometry after chemical treatment of tissue sections. Journal of the American Society for Mass Spectrometry 2008;19:1069-77

100. Thomas A, Patterson NH, Laveaux Charbonneau J, Chaurand P. Orthogonal organic and aqueous-based washes of tissue sections to enhance protein sensitivity by MALDI imaging mass spectrometry. Journal of mass spectrometry : JMS 2013;48:42-8

101. Gemperline E, Rawson S, Li L. Optimization and comparison of multiple MALDI matrix application methods for small molecule mass spectrometric imaging. Analytical chemistry 2014;86:10030-5

102. Yang J, Caprioli RM. Matrix precoated targets for direct lipid analysis and imaging of tissue. Analytical chemistry 2013;85:2907-12

103. Barre FP, Flinders B, Garcia JP, Jansen I, Huizing LR, Porta T, et al. Derivatization Strategies for the Detection of Triamcinolone Acetonide in Cartilage by Using Matrix-Assisted Laser Desorption/Ionization Mass Spectrometry Imaging. Analytical chemistry 2016;88:12051-9

104. Liu X, Hummon AB. Chemical Imaging of Platinum-Based Drugs and their Metabolites. Scientific reports 2016;6:38507

105. Franck J, Ayed ME, Wisztorski M, Salzet M, Fournier I. On tissue protein identification improvement by $\mathrm{N}$-terminal peptide derivatization. Methods in molecular biology 2010;656:323-38

106. Wu C, Ifa DR, Manicke NE, Cooks RG. Rapid, direct analysis of cholesterol by charge labeling in reactive desorption electrospray ionization. Analytical chemistry 2009;81:7618-24

107. Lemaire R, Desmons A, Tabet JC, Day R, Salzet M, Fournier I. Direct analysis and MALDI imaging of formalin-fixed, paraffin-embedded tissue sections. Journal of proteome research 2007;6:1295-305

108. Gustafsson JO, Oehler MK, McColl SR, Hoffmann P. Citric acid antigen retrieval (CAAR) for tryptic peptide imaging directly on archived formalin-fixed paraffinembedded tissue. Journal of proteome research 2010;9:4315-28

109. Heijs B, Carreira RJ, Tolner EA, de Ru AH, van den Maagdenberg AM, van Veelen $\mathrm{PA}$, et al. Comprehensive analysis of the mouse brain proteome sampled in mass spectrometry imaging. Analytical chemistry 2015;87:1867-75

110. Diehl HC, Beine B, Elm J, Trede D, Ahrens M, Eisenacher M, et al. The challenge of on-tissue digestion for MALDI MSI- a comparison of different protocols to improve imaging experiments. Analytical and bioanalytical chemistry 2015;407:2223-43

111. Heijs B, Tolner EA, Bovee JV, van den Maagdenberg AM, McDonnell LA. Brain Region-Specific Dynamics of On-Tissue Protein Digestion Using MALDI Mass Spectrometry Imaging. Journal of proteome research 2015;14:5348-54

112. Oetjen J, Lachmund D, Palmer A, Alexandrov T, Becker M, Boskamp T, et al. An approach to optimize sample preparation for MALDI imaging MS of FFPE sections 
using fractional factorial design of experiments. Analytical and bioanalytical chemistry 2016;408:6729-40

113. Erich K, Sammour DA, Marx A, Hopf C. Scores for standardization of on-tissue digestion of formalin-fixed paraffin-embedded tissue in MALDI-MS imaging. Biochimica et biophysica acta 2016

114. Powers TW, Neely BA, Shao Y, Tang H, Troyer DA, Mehta AS, et al. MALDI imaging mass spectrometry profiling of $\mathrm{N}$-glycans in formalin-fixed paraffin embedded clinical tissue blocks and tissue microarrays. PloS one 2014;9:e106255

115. Powers TW, Jones EE, Betesh LR, Romano PR, Gao P, Copland JA, et al. Matrix assisted laser desorption ionization imaging mass spectrometry workflow for spatial profiling analysis of $\mathrm{N}$-linked glycan expression in tissues. Analytical chemistry 2013;85:9799-806

116. Holst S, Heijs B, de Haan N, van Zeijl RJ, Briaire-de Bruijn IH, van Pelt GW, et al. Linkage-Specific in Situ Sialic Acid Derivatization for N-Glycan Mass Spectrometry Imaging of Formalin-Fixed Paraffin-Embedded Tissues. Analytical chemistry 2016;88:5904-13

117. Heijs B, Holst S, Briaire-de Bruijn IH, van Pelt GW, de Ru AH, van Veelen PA, et al. Multimodal Mass Spectrometry Imaging of N-Glycans and Proteins from the Same Tissue Section. Analytical chemistry 2016;88:7745-53

118. Buck A, Ly A, Balluff B, Sun N, Gorzolka K, Feuchtinger A, et al. High-resolution MALDI-FT-ICR MS imaging for the analysis of metabolites from formalin-fixed, paraffin-embedded clinical tissue samples. The Journal of pathology 2015;237:12332

119. Hatsis P, Brombacher S, Corr J, Kovarik P, Volmer DA. Quantitative analysis of small pharmaceutical drugs using a high repetition rate laser matrix-assisted laser/desorption ionization source. Rapid communications in mass spectrometry : RCM 2003;17:2303-9

120. Nilsson A, Goodwin RJ, Shariatgorji M, Vallianatou T, Webborn PJ, Andren PE. Mass spectrometry imaging in drug development. Analytical chemistry 2015;87:1437-55

121. Prideaux B, Stoeckli M. Mass spectrometry imaging for drug distribution studies. Journal of proteomics 2012;75:4999-5013

122. Prentice BM, Chumbley CW, Caprioli RM. Absolute Quantification of Rifampicin by MALDI Imaging Mass Spectrometry Using Multiple TOF/TOF Events in a Single Laser Shot. Journal of the American Society for Mass Spectrometry 2017;28:136-44

123. Taban IM, Altelaar AF, van der Burgt YE, McDonnell LA, Heeren RM, Fuchser J, et al. Imaging of peptides in the rat brain using MALDI-FTICR mass spectrometry. Journal of the American Society for Mass Spectrometry 2007;18:145-51

124. Rompp A, Guenther S, Takats Z, Spengler B. Mass spectrometry imaging with high resolution in mass and space (HR(2) MSI) for reliable investigation of drug compound distributions on the cellular level. Analytical and bioanalytical chemistry 2011;401:65-73

125. Rompp A, Spengler B. Mass spectrometry imaging with high resolution in mass and space. Histochemistry and cell biology 2013;139:759-83

126. Robichaud G, Barry JA, Garrard KP, Muddiman DC. Infrared matrix-assisted laser desorption electrospray ionization (IR-MALDESI) imaging source coupled to a FT- 
ICR mass spectrometer. Journal of the American Society for Mass Spectrometry 2013;24:92-100

127. Djidja MC, Claude E, Snel MF, Francese S, Scriven P, Carolan V, et al. Novel molecular tumour classification using MALDI-mass spectrometry imaging of tissue micro-array. Analytical and bioanalytical chemistry 2010;397:587-601

128. Groseclose MR, Massion PP, Chaurand P, Caprioli RM. High-throughput proteomic analysis of formalin-fixed paraffin-embedded tissue microarrays using MALDI imaging mass spectrometry. Proteomics 2008;8:3715-24

129. Pirman DA, Yost RA. Quantitative tandem mass spectrometric imaging of endogenous acetyl-L-carnitine from piglet brain tissue using an internal standard. Analytical chemistry 2011;83:8575-81

130. Hopfgartner G, Varesio E, Stoeckli M. Matrix-assisted laser desorption/ionization mass spectrometric imaging of complete rat sections using a triple quadrupole linear ion trap. Rapid communications in mass spectrometry : RCM 2009;23:733-6

131. Porta T, Grivet C, Kraemer T, Varesio E, Hopfgartner G. Single hair cocaine consumption monitoring by mass spectrometric imaging. Analytical chemistry 2011;83:4266-72

132. Prideaux B, Dartois V, Staab D, Weiner DM, Goh A, Via LE, et al. High-sensitivity MALDI-MRM-MS imaging of moxifloxacin distribution in tuberculosis-infected rabbit lungs and granulomatous lesions. Analytical chemistry 2011;83:2112-8

133. Fisher GL, Bruinen AL, Ogrinc Potocnik N, Hammond JS, Bryan SR, Larson PE, et al. A New Method and Mass Spectrometer Design for TOF-SIMS Parallel Imaging MS/MS. Analytical chemistry 2016;88:6433-40

134. Jackson SN, Ugarov M, Post JD, Egan T, Langlais D, Schultz JA, et al. A study of phospholipids by ion mobility TOFMS. Journal of the American Society for Mass Spectrometry 2008;19:1655-62

135. Chouinard CD, Wei MS, Beekman CR, Kemperman RH, Yost RA. Ion Mobility in Clinical Analysis: Current Progress and Future Perspectives. Clinical chemistry 2016;62:124-33

136. Porta T, Varesio E, Hopfgartner G. Gas-phase separation of drugs and metabolites using modifier-assisted differential ion mobility spectrometry hyphenated to liquid extraction surface analysis and mass spectrometry. Analytical chemistry 2013;85:11771-9

137. Chughtai K, Jiang L, Greenwood TR, Glunde K, Heeren RM. Mass spectrometry images acylcarnitines, phosphatidylcholines, and sphingomyelin in MDA-MB-231 breast tumor models. Journal of lipid research 2013;54:333-44

138. Skraskova K, Claude E, Jones EA, Towers M, Ellis SR, Heeren RM. Enhanced capabilities for imaging gangliosides in murine brain with matrix-assisted laser desorption/ionization and desorption electrospray ionization mass spectrometry coupled to ion mobility separation. Methods 2016;104:69-78

139. Roux A, Muller L, Jackson SN, Post J, Baldwin K, Hoffer B, et al. Mass spectrometry imaging of rat brain lipid profile changes over time following traumatic brain injury. Journal of neuroscience methods 2016;272:19-32

140. Cole LM, Mahmoud K, Haywood-Small S, Tozer GM, Smith DP, Clench MR. Recombinant "IMS TAG" proteins--a new method for validating bottom-up matrix- 
assisted laser desorption/ionisation ion mobility separation mass spectrometry imaging. Rapid communications in mass spectrometry : RCM 2013;27:2355-62

141. Djidja MC, Francese S, Loadman PM, Sutton CW, Scriven P, Claude E, et al. Detergent addition to tryptic digests and ion mobility separation prior to MS/MS improves peptide yield and protein identification for in situ proteomic investigation of frozen and formalin-fixed paraffin-embedded adenocarcinoma tissue sections. Proteomics 2009;9:2750-63

142. Griffiths RL, Creese AJ, Race AM, Bunch J, Cooper HJ. LESA FAIMS Mass Spectrometry for the Spatial Profiling of Proteins from Tissue. Analytical chemistry 2016;88:6758-66

143. Sarsby J, Griffiths RL, Race AM, Bunch J, Randall EC, Creese AJ, et al. Liquid Extraction Surface Analysis Mass Spectrometry Coupled with Field Asymmetric Waveform Ion Mobility Spectrometry for Analysis of Intact Proteins from Biological Substrates. Analytical chemistry 2015;87:6794-800

144. Terral G, Beck A, Cianferani S. Insights from native mass spectrometry and ion mobility-mass spectrometry for antibody and antibody-based product characterization. Journal of chromatography B, Analytical technologies in the biomedical and life sciences 2016;1032:79-90

145. Feider CL, Elizondo N, Eberlin LS. Ambient lonization and FAIMS Mass Spectrometry for Enhanced Imaging of Multiply Charged Molecular Ions in Biological Tissues. Analytical chemistry 2016;88:11533-41

146. Soltwisch J, Kettling H, Vens-Cappell S, Wiegelmann M, Muthing J, Dreisewerd K. Mass spectrometry imaging with laser-induced postionization. Science 2015;348:211-5

147. Maier SK, Hahne H, Gholami AM, Balluff B, Meding S, Schoene C, et al. Comprehensive identification of proteins from MALDI imaging. Molecular \& cellular proteomics : MCP 2013;12:2901-10

148. Rauser S, Marquardt C, Balluff B, Deininger SO, Albers C, Belau E, et al. Classification of HER2 receptor status in breast cancer tissues by MALDI imaging mass spectrometry. Journal of proteome research 2010;9:1854-63

149. Spraggins JM, Rizzo DG, Moore JL, Rose KL, Hammer ND, Skaar EP, et al. MALDI FTICR IMS of Intact Proteins: Using Mass Accuracy to Link Protein Images with Proteomics Data. Journal of the American Society for Mass Spectrometry 2015;26:974-85

150. McDonnell LA, Walch A, Stoeckli M, Corthals GL. MSiMass list: a public database of identifications for protein MALDI MS imaging. Journal of proteome research 2014;13:1138-42

151. Schober Y, Schramm T, Spengler B, Rompp A. Protein identification by accurate mass matrix-assisted laser desorption/ionization imaging of tryptic peptides. Rapid communications in mass spectrometry : RCM 2011;25:2475-83

152. Groseclose MR, Andersson M, Hardesty WM, Caprioli RM. Identification of proteins directly from tissue: in situ tryptic digestions coupled with imaging mass spectrometry. Journal of mass spectrometry : JMS 2007;42:254-62

153. Ye H, Hui L, Kellersberger K, Li L. Mapping of neuropeptides in the crustacean stomatogastric nervous system by imaging mass spectrometry. Journal of the American Society for Mass Spectrometry 2013;24:134-47 
154. Gustafsson OJ, Eddes JS, Meding S, McColl SR, Oehler MK, Hoffmann P. Matrixassisted laser desorption/ionization imaging protocol for in situ characterization of tryptic peptide identity and distribution in formalin-fixed tissue. Rapid communications in mass spectrometry : RCM 2013;27:655-70

155. Jones EE, Powers TW, Neely BA, Cazares LH, Troyer DA, Parker AS, et al. MALDI imaging mass spectrometry profiling of proteins and lipids in clear cell renal cell carcinoma. Proteomics 2014;14:924-35

156. Giordano S, Morosi L, Veglianese P, Licandro SA, Frapolli R, Zucchetti M, et al. 3D Mass Spectrometry Imaging Reveals a Very Heterogeneous Drug Distribution in Tumors. Scientific reports 2016;6:37027

157. Watrous JD, Phelan VV, Hsu CC, Moree WJ, Duggan BM, Alexandrov T, et al. Microbial metabolic exchange in 3D. The ISME journal 2013;7:770-80

158. Lotz JM, Hoffmann F, Lotz J, Heldmann S, Trede D, Oetjen J, et al. Integration of 3D multimodal imaging data of a head and neck cancer and advanced feature recognition. Biochimica et biophysica acta 2016

159. Xiong $\mathrm{X}, \mathrm{Xu} \mathrm{W}$, Eberlin $\mathrm{LS}$, Wiseman JM, Fang $\mathrm{X}$, Jiang $\mathrm{Y}$, et al. Data processing for 3D mass spectrometry imaging. Journal of the American Society for Mass Spectrometry 2012;23:1147-56

160. Vanbellingen QP, Castellanos A, Rodriguez-Silva M, Paudel I, Chambers JW, Fernandez-Lima FA. Analysis of Chemotherapeutic Drug Delivery at the Single Cell Level Using 3D-MSI-TOF-SIMS. Journal of the American Society for Mass Spectrometry 2016;27:2033-40

161. Fletcher JS, Lockyer NP, Vickerman JC. Developments in molecular SIMS depth profiling and 3D imaging of biological systems using polyatomic primary ions. Mass spectrometry reviews 2011;30:142-74

162. Fisher GL, Belu AM, Mahoney CM, Wormuth K, Sanada N. Three-dimensional timeof-flight secondary ion mass spectrometry imaging of a pharmaceutical in a coronary stent coating as a function of elution time. Analytical chemistry 2009;81:9930-40

163. Fletcher JS, Vickerman JC. A new SIMS paradigm for 2D and 3D molecular imaging of bio-systems. Analytical and bioanalytical chemistry 2010;396:85-104

164. Van Nuffel S, Parmenter C, Scurr DJ, Russell NA, Zelzer M. Multivariate analysis of 3D ToF-SIMS images: method validation and application to cultured neuronal networks. The Analyst 2016;141:90-5

165. Verbeeck N, Spraggins JM, Murphy MJ, Wang HD, Deutch AY, Caprioli RM, et al. Connecting Imaging Mass Spectrometry and Magnetic Resonance Imaging-based Anatomical Atlases for Automated Anatomical Interpretation and Differential Analysis. Biochimica et biophysica acta 2017

166. Chughtai K, Jiang L, Post H, Winnard PT, Jr., Greenwood TR, Raman V, et al. Mass spectrometric imaging of red fluorescent protein in breast tumor xenografts. Journal of the American Society for Mass Spectrometry 2013;24:711-7

167. Patterson NH, Doonan RJ, Daskalopoulou SS, Dufresne M, Lenglet S, Montecucco $\mathrm{F}$, et al. Three-dimensional imaging MS of lipids in atherosclerotic plaques: Opensource methods for reconstruction and analysis. Proteomics 2016;16:1642-51 
168. Robichaud G, Garrard KP, Barry JA, Muddiman DC. MSiReader: an open-source interface to view and analyze high resolving power MS imaging files on Matlab platform. Journal of the American Society for Mass Spectrometry 2013;24:718-21

169. Bemis KD, Harry A, Eberlin LS, Ferreira C, van de Ven SM, Mallick P, et al. Cardinal: an $\mathrm{R}$ package for statistical analysis of mass spectrometry-based imaging experiments. Bioinformatics 2015;31:2418-20

170. Robbe MF, Both JP, Prideaux B, Klinkert I, Picaud V, Schramm T, et al. Software tools of the Computis European project to process mass spectrometry images. European journal of mass spectrometry 2014;20:351-60

171. Kallback P, Nilsson A, Shariatgorji M, Andren PE. msIQuant--Quantitation Software for Mass Spectrometry Imaging Enabling Fast Access, Visualization, and Analysis of Large Data Sets. Analytical chemistry 2016;88:4346-53

172. Race AM, Palmer AD, Dexter A, Steven RT, Styles IB, Bunch J. SpectralAnalysis: Software for the Masses. Analytical chemistry 2016;88:9451-8

173. Van de Plas R, Yang J, Spraggins J, Caprioli RM. Image fusion of mass spectrometry and microscopy: a multimodality paradigm for molecular tissue mapping. Nature methods 2015;12:366-72

174. Cassese A, Ellis SR, Ogrinc Potocnik N, Burgermeister E, Ebert M, Walch A, et al. Spatial Autocorrelation in Mass Spectrometry Imaging. Analytical chemistry 2016;88:5871-8

175. Widlak P, Mrukwa G, Kalinowska M, Pietrowska M, Chekan M, Wierzgon J, et al. Detection of molecular signatures of oral squamous cell carcinoma and normal epithelium - application of a novel methodology for unsupervised segmentation of imaging mass spectrometry data. Proteomics 2016;16:1613-21

176. Alexandrov T, Kobarg JH. Efficient spatial segmentation of large imaging mass spectrometry datasets with spatially aware clustering. Bioinformatics 2011;27:i230-8

177. Palmer A, Phapale P, Chernyavsky I, Lavigne R, Fay D, Tarasov A, et al. FDRcontrolled metabolite annotation for high-resolution imaging mass spectrometry. Nature methods 2017; 14:57-60

178. Schramm T, Hester A, Klinkert I, Both JP, Heeren RM, Brunelle A, et al. imzML--a common data format for the flexible exchange and processing of mass spectrometry imaging data. Journal of proteomics 2012;75:5106-10

179. Rompp A, Wang R, Albar JP, Urbani A, Hermjakob H, Spengler B, et al. A public repository for mass spectrometry imaging data. Analytical and bioanalytical chemistry 2015;407:2027-33

180. McDonnell LA, Rompp A, Balluff B, Heeren RM, Albar JP, Andren PE, et al. Discussion point: reporting guidelines for mass spectrometry imaging. Analytical and bioanalytical chemistry 2015;407:2035-45

181. Lou S, Balluff B, Cleven AH, Bovee JV, McDonnell LA. An experimental guideline for the analysis of histologically heterogeneous tumors by MALDI-TOF mass spectrometry imaging. Biochimica et biophysica acta 2016

182. Lemaire R, Menguellet SA, Stauber J, Marchaudon V, Lucot JP, Collinet P, et al. Specific MALDI imaging and profiling for biomarker hunting and validation: fragment of the $11 \mathrm{~S}$ proteasome activator complex, Reg alpha fragment, is a new potential ovary cancer biomarker. Journal of proteome research 2007;6:4127-34 
183. Longuespee R, Boyon C, Castellier C, Jacquet A, Desmons A, Kerdraon O, et al. The C-terminal fragment of the immunoproteasome PA28S (Reg alpha) as an early diagnosis and tumor-relapse biomarker: evidence from mass spectrometry profiling. Histochemistry and cell biology 2012;138:141-54

184. Cazares LH, Troyer D, Mendrinos S, Lance RA, Nyalwidhe JO, Beydoun HA, et al. Imaging mass spectrometry of a specific fragment of mitogen-activated protein kinase/extracellular signal-regulated kinase kinase kinase 2 discriminates cancer from uninvolved prostate tissue. Clinical cancer research : an official journal of the American Association for Cancer Research 2009;15:5541-51

185. Elsner M, Rauser S, Maier S, Schone C, Balluff B, Meding S, et al. MALDI imaging mass spectrometry reveals COX7A2, TAGLN2 and S100-A10 as novel prognostic markers in Barrett's adenocarcinoma. Journal of proteomics 2012;75:4693-704

186. Laouirem S, Le Faouder J, Alexandrov T, Mestivier D, Leger T, Baudin X, et al. Progression from cirrhosis to cancer is associated with early ubiquitin posttranslational modifications: identification of new biomarkers of cirrhosis at risk of malignancy. The Journal of pathology 2014;234:452-63

187. Rebours V, Le Faouder J, Laouirem S, Mebarki M, Albuquerque M, Camadro JM, et al. In situ proteomic analysis by MALDI imaging identifies ubiquitin and thymosinbeta4 as markers of malignant intraductal pancreatic mucinous neoplasms. Pancreatology : official journal of the International Association of Pancreatology 2014;14:117-24

188. Balluff B, Elsner M, Kowarsch A, Rauser S, Meding S, Schuhmacher C, et al. Classification of HER2/neu status in gastric cancer using a breast-cancer derived proteome classifier. Journal of proteome research 2010;9:6317-22

189. Meding S, Balluff B, Elsner M, Schone C, Rauser S, Nitsche U, et al. Tissue-based proteomics reveals FXYD3, S100A11 and GSTM3 as novel markers for regional lymph node metastasis in colon cancer. The Journal of pathology 2012;228:459-70

190. Deininger SO, Ebert MP, Futterer A, Gerhard M, Rocken C. MALDI imaging combined with hierarchical clustering as a new tool for the interpretation of complex human cancers. Journal of proteome research 2008;7:5230-6

191. Lazova R, Seeley EH, Keenan M, Gueorguieva R, Caprioli RM. Imaging mass spectrometry--a new and promising method to differentiate Spitz nevi from Spitzoid malignant melanomas. The American Journal of dermatopathology 2012;34:82-90

192. Aichler M, Elsner M, Ludyga N, Feuchtinger A, Zangen V, Maier SK, et al. Clinical response to chemotherapy in oesophageal adenocarcinoma patients is linked to defects in mitochondria. The Journal of pathology 2013;230:410-9

193. Wiseman JM, Ifa DR, Song Q, Cooks RG. Tissue imaging at atmospheric pressure using desorption electrospray ionization (DESI) mass spectrometry. Angewandte Chemie 2006;45:7188-92

194. Dill AL, Ifa DR, Manicke NE, Costa AB, Ramos-Vara JA, Knapp DW, et al. Lipid profiles of canine invasive transitional cell carcinoma of the urinary bladder and adjacent normal tissue by desorption electrospray ionization imaging mass spectrometry. Analytical chemistry 2009;81:8758-64 
195. Eberlin LS, Norton I, Dill AL, Golby AJ, Ligon KL, Santagata S, et al. Classifying human brain tumors by lipid imaging with mass spectrometry. Cancer research 2012;72:645-54

196. Guenther S, Muirhead U, Speller AV, Golf O, Strittmatter N, Ramakrishnan R, et al. Spatially resolved metabolic phenotyping of breast cancer by desorption electrospray ionization mass spectrometry. Cancer research 2015;75:1828-37

197. Abbassi-Ghadi N, Golf O, Kumar S, Antonowicz S, McKenzie JS, Huang J, et al. Imaging of Esophageal Lymph Node Metastases by Desorption Electrospray Ionization Mass Spectrometry. Cancer research 2016;76:5647-56

198. Balluff B, Rauser S, Meding S, Elsner M, Schone C, Feuchtinger A, et al. MALDI imaging identifies prognostic seven-protein signature of novel tissue markers in intestinal-type gastric cancer. The American journal of pathology 2011;179:2720-9

199. Gemoll T, Strohkamp S, Schillo K, Thorns C, Habermann JK. MALDI-imaging reveals thymosin beta-4 as an independent prognostic marker for colorectal cancer. Oncotarget 2015;6:43869-80

200. Lou S, Balluff B, de Graaff MA, Cleven AH, Briaire-de Bruijn I, Bovée JV, et al. Highgrade sarcoma diagnosis and prognosis: Biomarker discovery by mass spectrometry imaging. Proteomics 2016;16:1802-13

201. Chung L, Phillips L, Lin MZ, Moore K, Marsh DJ, Boyle FM, et al. A novel truncated form of S100P predicts disease-free survival in patients with lymph node positive breast cancer. Cancer letters 2015;368:64-70

202. Hardesty WM, Kelley MC, Mi D, Low RL, Caprioli RM. Protein signatures for survival and recurrence in metastatic melanoma. Journal of proteomics 2011;74:1002-14

203. Pote N, Alexandrov T, Le Faouder J, Laouirem S, Leger T, Mebarki M, et al. Imaging mass spectrometry reveals modified forms of histone $\mathrm{H} 4$ as new biomarkers of microvascular invasion in hepatocellular carcinomas. Hepatology (Baltimore, Md) 2013;58:983-94

204. Bauer JA, Chakravarthy AB, Rosenbluth JM, Mi D, Seeley EH, De Matos GranjaIngram $\mathrm{N}$, et al. Identification of markers of taxane sensitivity using proteomic and genomic analyses of breast tumors from patients receiving neoadjuvant paclitaxel and radiation. Clinical cancer research : an official journal of the American Association for Cancer Research 2010;16:681-90

205. Lazova R, Seeley EH, Kutzner H, Scolyer RA, Scott G, Cerroni L, et al. Imaging mass spectrometry assists in the classification of diagnostically challenging atypical Spitzoid neoplasms. Journal of the American Academy of Dermatology 2016;75:1176-86 e4

206. Balluff B, Frese CK, Maier SK, Schone C, Kuster B, Schmitt M, et al. De novo discovery of phenotypic intratumour heterogeneity using imaging mass spectrometry. The Journal of pathology 2015;235:3-13

207. Mulder IA, Esteve C, Wermer MJ, Hoehn M, Tolner EA, van den Maagdenberg AM, et al. Funnel-freezing versus heat-stabilization for the visualization of metabolites by mass spectrometry imaging in a mouse stroke model. Proteomics 2016;16:1652-9

208. Gerbig S, Golf O, Balog J, Denes J, Baranyai Z, Zarand A, et al. Analysis of colorectal adenocarcinoma tissue by desorption electrospray ionization mass spectrometric imaging. Analytical and bioanalytical chemistry 2012;403:2315-25 
209. Patterson NH, Alabdulkarim B, Lazaris A, Thomas A, Marcinkiewicz MM, Gao ZH, et al. Assessment of pathological response to therapy using lipid mass spectrometry imaging. Scientific reports 2016;6:36814

210. Lou S, Balluff B, Cleven AH, Bovee JV, McDonnell LA. Prognostic Metabolite Biomarkers for Soft Tissue Sarcomas Discovered by Mass Spectrometry Imaging. Journal of the American Society for Mass Spectrometry 2017;28:376-83

211. Mirnezami R, Spagou K, Vorkas PA, Lewis MR, Kinross J, Want E, et al. Chemical mapping of the colorectal cancer microenvironment via MALDI imaging mass spectrometry (MALDI-MSI) reveals novel cancer-associated field effects. Molecular oncology 2014;8:39-49

212. Oppenheimer SR, Mi D, Sanders ME, Caprioli RM. Molecular analysis of tumor margins by MALDI mass spectrometry in renal carcinoma. Journal of proteome research 2010;9:2182-90

213. Calligaris D, Feldman DR, Norton I, Brastianos PK, Dunn IF, Santagata S, et al. Molecular typing of Meningiomas by Desorption Electrospray Ionization Mass Spectrometry Imaging for Surgical Decision-Making. International journal of mass spectrometry 2015;377:690-8

214. Calligaris D, Caragacianu D, Liu X, Norton I, Thompson CJ, Richardson AL, et al. Application of desorption electrospray ionization mass spectrometry imaging in breast cancer margin analysis. Proceedings of the National Academy of Sciences of the United States of America 2014;111:15184-9

215. Eberlin LS, Tibshirani RJ, Zhang J, Longacre TA, Berry GJ, Bingham DB, et al. Molecular assessment of surgical-resection margins of gastric cancer by massspectrometric imaging. Proceedings of the National Academy of Sciences of the United States of America 2014;111:2436-41

216. Eberlin LS, Margulis K, Planell-Mendez I, Zare RN, Tibshirani R, Longacre TA, et al. Pancreatic Cancer Surgical Resection Margins: Molecular Assessment by Mass Spectrometry Imaging. PLoS medicine 2016;13:e1002108

217. Tata A, Zheng J, Ginsberg HJ, Jaffray DA, Ifa DR, Zarrine-Afsar A. Contrast Agent Mass Spectrometry Imaging Reveals Tumor Heterogeneity. Analytical chemistry 2015;87:7683-9

218. Abbassi-Ghadi N, Veselkov K, Kumar S, Huang J, Jones E, Strittmatter N, et al. Discrimination of lymph node metastases using desorption electrospray ionisationmass spectrometry imaging. Chemical communications 2014;50:3661-4

219. Kerian KS, Jarmusch AK, Cooks RG. Touch spray mass spectrometry for in situ analysis of complex samples. The Analyst 2014;139:2714-20

220. Kerian KS, Jarmusch AK, Pirro V, Koch MO, Masterson TA, Cheng L, et al. Differentiation of prostate cancer from normal tissue in radical prostatectomy specimens by desorption electrospray ionization and touch spray ionization mass spectrometry. The Analyst 2015;140:1090-8

221. Alfaro CM, Jarmusch AK, Pirro V, Kerian KS, Masterson TA, Cheng L, et al. Ambient ionization mass spectrometric analysis of human surgical specimens to distinguish renal cell carcinoma from healthy renal tissue. Analytical and bioanalytical chemistry 2016;408:5407-14 
222. Balog J, Sasi-Szabo L, Kinross J, Lewis MR, Muirhead LJ, Veselkov K, et al. Intraoperative tissue identification using rapid evaporative ionization mass spectrometry. Science translational medicine 2013;5:194ra93

223. Alexander J, Gildea L, Balog J, Speller A, McKenzie J, Muirhead L, et al. A novel methodology for in vivo endoscopic phenotyping of colorectal cancer based on real-time analysis of the mucosal lipidome: a prospective observational study of the iKnife. Surgical endoscopy 2017;31:1361-70

224. Balog J, Kumar S, Alexander J, Golf O, Huang J, Wiggins T, et al. In vivo endoscopic tissue identification by rapid evaporative ionization mass spectrometry (REIMS). Angewandte Chemie 2015;54:11059-62

225. Fatou B, Saudemont $P$, Leblanc E, Vinatier D, Mesdag V, Wisztorski M, et al. In vivo Real-Time Mass Spectrometry for Guided Surgery Application. Scientific reports 2016;6:25919

226. Pirro V, Eberlin LS, Oliveri P, Cooks RG. Interactive hyperspectral approach for exploring and interpreting DESI-MS images of cancerous and normal tissue sections. The Analyst 2012;137:2374-80

227. Meding S, Nitsche U, Balluff B, Elsner M, Rauser S, Schone C, et al. Tumor classification of six common cancer types based on proteomic profiling by MALDI imaging. Journal of proteome research 2012;11:1996-2003

228. Eberlin LS, Gabay M, Fan AC, Gouw AM, Tibshirani RJ, Felsher DW, et al. Alteration of the lipid profile in lymphomas induced by MYC overexpression. Proceedings of the National Academy of Sciences of the United States of America 2014;111:10450-5

229. Gruner BM, Hahne H, Mazur PK, Trajkovic-Arsic M, Maier S, Esposito I, et al. MALDI imaging mass spectrometry for in situ proteomic analysis of preneoplastic lesions in pancreatic cancer. PloS one 2012; 7:e39424

230. Grüner BM, Winkelmann I, Feuchtinger A, Sun N, Balluff B, Teichmann N, et al. Modeling Therapy Response and Spatial Tissue Distribution of Erlotinib in Pancreatic Cancer. Molecular cancer therapeutics 2016;15:1145-52

231. Rubio DM, Schoenbaum EE, Lee LS, Schteingart DE, Marantz PR, Anderson KE, et al. Defining translational research: implications for training. Academic medicine : journal of the Association of American Medical Colleges 2010;85:470-5

232. Desbenoit N, Schmitz-Afonso I, Baudouin C, Laprevote O, Touboul D, BrignoleBaudouin $\mathrm{F}$, et al. Localisation and quantification of benzalkonium chloride in eye tissue by TOF-SIMS imaging and liquid chromatography mass spectrometry. Analytical and bioanalytical chemistry 2013;405:4039-49

233. Groseclose MR, Laffan SB, Frazier KS, Hughes-Earle A, Castellino S. Imaging MS in Toxicology: An Investigation of Juvenile Rat Nephrotoxicity Associated with Dabrafenib Administration. Journal of the American Society for Mass Spectrometry 2015;26:887-98

234. Yoshimi T, Kawabata S, Taira S, Okuno A, Mikawa R, Murayama S, et al. Affinity imaging mass spectrometry (AIMS): high-throughput screening for specific small molecule interactions with frozen tissue sections. The Analyst 2015;140:7202-8

235. Hanrieder J, Ewing AG. Spatial elucidation of spinal cord lipid-and metaboliteregulations in amyotrophic lateral sclerosis. Scientific reports 2014;4:5266 
236. Lazar AN, Bich C, Panchal M, Desbenoit N, Petit VW, Touboul D, et al. Time-of-flight secondary ion mass spectrometry (TOF-SIMS) imaging reveals cholesterol overload in the cerebral cortex of Alzheimer disease patients. Acta neuropathologica 2013;125:133-44

237. Mendis LH, Grey AC, Faull RL, Curtis MA. Hippocampal lipid differences in Alzheimer's disease: a human brain study using matrix-assisted laser desorption/ionization-imaging mass spectrometry. Brain and behavior 2016;6:e00517

238. Carreira RJ, Shyti R, Balluff B, Abdelmoula WM, van Heiningen SH, van Zeijl RJ, et al. Large-scale mass spectrometry imaging investigation of consequences of cortical spreading depression in a transgenic mouse model of migraine. Journal of the American Society for Mass Spectrometry 2015;26:853-61

239. Sarkis GA, Mangaonkar MD, Moghieb A, Lelling B, Guertin M, Yadikar H, et al. The Application of Proteomics to Traumatic Brain and Spinal Cord Injuries. Current neurology and neuroscience reports 2017;17:23

240. Ljungdahl A, Hanrieder J, Falth M, Bergquist J, Andersson M. Imaging mass spectrometry reveals elevated nigral levels of dynorphin neuropeptides in L-DOPAinduced dyskinesia in rat model of Parkinson's disease. PloS one 2011;6:e25653

241. Skold K, Svensson M, Nilsson A, Zhang X, Nydahl K, Caprioli RM, et al. Decreased striatal levels of PEP-19 following MPTP lesion in the mouse. Journal of proteome research 2006;5:262-9

242. Pierson J, Norris JL, Aerni HR, Svenningsson P, Caprioli RM, Andren PE. Molecular profiling of experimental Parkinson's disease: direct analysis of peptides and proteins on brain tissue sections by MALDI mass spectrometry. Journal of proteome research 2004;3:289-95

243. Matsumoto J, Sugiura Y, Yuki D, Hayasaka T, Goto-Inoue N, Zaima N, et al. Abnormal phospholipids distribution in the prefrontal cortex from a patient with schizophrenia revealed by matrix-assisted laser desorption/ionization imaging mass spectrometry. Analytical and bioanalytical chemistry 2011;400:1933-43

244. Dufresne M, Guneysu D, Patterson NH, Marcinkiewicz MM, Regina A, Demeule M, et al. Multimodal detection of GM2 and GM3 lipid species in the brain of mucopolysaccharidosis type II mouse by serial imaging mass spectrometry and immunohistochemistry. Analytical and bioanalytical chemistry 2017;409:1425-33

245. Llombart V, Trejo SA, Bronsoms S, Morancho A, Feifei M, Faura J, et al. Profiling and identification of new proteins involved in brain ischemia using MALDI-imagingmass-spectrometry. Journal of proteomics 2017;152:243-53

246. Shariatgorji M, Svenningsson P, Andren PE. Mass spectrometry imaging, an emerging technology in neuropsychopharmacology. Neuropsychopharmacology : official publication of the American College of Neuropsychopharmacology 2014;39:34-49

247. Schubert KO, Weiland F, Baune BT, Hoffmann P. The use of MALDI-MSI in the investigation of psychiatric and neurodegenerative disorders: A review. Proteomics 2016;16:1747-58

248. Martin-Lorenzo M, Balluff B, Maroto AS, Carreira RJ, van Zeijl RJ, Gonzalez-Calero $\mathrm{L}$, et al. Molecular anatomy of ascending aorta in atherosclerosis by MS Imaging: 
Specific lipid and protein patterns reflect pathology. Journal of proteomics 2015;126:245-51

249. Martin-Lorenzo M, Alvarez-Llamas G, McDonnell LA, Vivanco F. Molecular histology of arteries: mass spectrometry imaging as a novel ex vivo tool to investigate atherosclerosis. Expert review of proteomics 2016;13:69-81

250. Aichler M, Huber K, Schilling F, Lohofer F, Kosanke K, Meier R, et al. Spatially resolved quantification of gadolinium(III)-based magnetic resonance agents in tissue by MALDI imaging mass spectrometry after in vivo MRI. Angewandte Chemie 2015;54:4279-83

251. Mourino-Alvarez L, Iloro I, de la Cuesta F, Azkargorta M, Sastre-Oliva T, Escobes I, et al. MALDI-Imaging Mass Spectrometry: a step forward in the anatomopathological characterization of stenotic aortic valve tissue. Scientific reports 2016;6:27106

252. Angel PM, Baldwin HS, Gottlieb Sen D, Su YR, Mayer JE, Bichell D, et al. Advances in MALDI imaging mass spectrometry of proteins in cardiac tissue, including the heart valve. Biochimica et biophysica acta 2017

253. Rocha B, Ruiz-Romero C, Blanco FJ. Mass spectrometry imaging: a novel technology in rheumatology. Nature reviews Rheumatology 2017;13:52-63

254. Briggs MT, Kuliwaba JS, Muratovic D, Everest-Dass AV, Packer NH, Findlay DM, et al. MALDI mass spectrometry imaging of $\mathrm{N}$-glycans on tibial cartilage and subchondral bone proteins in knee osteoarthritis. Proteomics 2016;16:1736-41

255. Cillero-Pastor B, Eijkel G, Kiss A, Blanco FJ, Heeren RM. Time-of-flight secondary ion mass spectrometry-based molecular distribution distinguishing healthy and osteoarthritic human cartilage. Analytical chemistry 2012;84:8909-16

256. Cillero-Pastor B, Eijkel GB, Blanco FJ, Heeren RM. Protein classification and distribution in osteoarthritic human synovial tissue by matrix-assisted laser desorption ionization mass spectrometry imaging. Analytical and bioanalytical chemistry 2015;407:2213-22

257. Rocha B, Cillero-Pastor B, Eijkel G, Bruinen AL, Ruiz-Romero C, Heeren RM, et al. Characterization of lipidic markers of chondrogenic differentiation using mass spectrometry imaging. Proteomics 2015;15:702-13

258. Georgi N, Cillero-Pastor B, Eijkel GB, Periyasamy PC, Kiss A, van Blitterswijk C, et al. Differentiation of mesenchymal stem cells under hypoxia and normoxia: lipid profiles revealed by time-of-flight secondary ion mass spectrometry and multivariate analysis. Analytical chemistry 2015;87:3981-8

259. Peffers MJ, Cillero-Pastor B, Eijkel GB, Clegg PD, Heeren RM. Matrix assisted laser desorption ionization mass spectrometry imaging identifies markers of ageing and osteoarthritic cartilage. Arthritis research \& therapy 2014;16:R110

260. Mainini V, Pagni F, Ferrario F, Pieruzzi F, Grasso M, Stella A, et al. MALDI imaging mass spectrometry in glomerulonephritis: feasibility study. Histopathology 2014; 64:901-6

261. Smith A, L'Imperio V, De Sio G, Ferrario F, Scalia C, Dell'Antonio G, et al. alpha-1Antitrypsin detected by MALDI imaging in the study of glomerulonephritis: Its relevance in chronic kidney disease progression. Proteomics 2016;16:1759-66

262. Casadonte R, Kriegsmann M, Deininger SO, Amann K, Paape R, Belau E, et al. Imaging mass spectrometry analysis of renal amyloidosis biopsies reveals protein 
co-localization with amyloid deposits. Analytical and bioanalytical chemistry 2015;407:5323-31

263. Winter M, Tholey A, Kruger S, Schmidt H, Rocken C. MALDI-mass spectrometry imaging identifies vitronectin as a common constituent of amyloid deposits. The journal of histochemistry and cytochemistry : official journal of the Histochemistry Society 2015;63:772-9

264. Bowrey HE, Anderson DM, Pallitto P, Gutierrez DB, Fan J, Crouch RK, et al. Imaging mass spectrometry of the visual system: Advancing the molecular understanding of retina degenerations. Proteomics Clinical applications 2016;10:391-402

265. Crouch RK, Koutalos Y, Kono M, Schey K, Ablonczy Z. A2E and Lipofuscin. Progress in molecular biology and translational science 2015;134:449-63

266. Yakovleva MA, Gulin AA, Feldman TB, Bel'skich YC, Arbukhanova PM, Astaf'ev AA, et al. Time-of-flight secondary ion mass spectrometry to assess spatial distribution of $\mathrm{A} 2 \mathrm{E}$ and its oxidized forms within lipofuscin granules isolated from human retinal pigment epithelium. Analytical and bioanalytical chemistry 2016;408:75218

267. Anderson DM, Ablonczy Z, Koutalos Y, Spraggins J, Crouch RK, Caprioli RM, et al. High resolution MALDI imaging mass spectrometry of retinal tissue lipids. Journal of the American Society for Mass Spectrometry 2014;25:1394-403

268. Brignole-Baudouin F, Desbenoit N, Hamm G, Liang H, Both JP, Brunelle A, et al. A new safety concern for glaucoma treatment demonstrated by mass spectrometry imaging of benzalkonium chloride distribution in the eye, an experimental study in rabbits. PloS one 2012; 7:e50180

269. Nye-Wood MG, Spraggins JM, Caprioli RM, Schey KL, Donaldson PJ, Grey AC. Spatial distributions of glutathione and its endogenous conjugates in normal bovine lens and a model of lens aging. Experimental eye research 2017;154:70-8

270. Sun N, Ly A, Meding S, Witting M, Hauck SM, Ueffing $M$, et al. High-resolution metabolite imaging of light and dark treated retina using MALDI-FTICR mass spectrometry. Proteomics 2014;14:913-23

271. Taverna D, Pollins AC, Sindona G, Caprioli RM, Nanney LB. Imaging mass spectrometry for assessing cutaneous wound healing: analysis of pressure ulcers. Journal of proteome research 2015;14:986-96

272. Taverna D, Pollins AC, Sindona G, Caprioli RM, Nanney LB. Imaging mass spectrometry for accessing molecular changes during burn wound healing. Wound repair and regeneration : official publication of the Wound Healing Society [and] the European Tissue Repair Society 2016;24:775-85

273. Bowlus CL, Seeley EH, Roder J, Grigorieva J, Roder H, Caprioli RM, et al. In situ mass spectrometry of autoimmune liver diseases. Cellular \& molecular immunology 2011;8:237-42

274. Clarke R, Ressom HW, Wang A, Xuan J, Liu MC, Gehan EA, et al. The properties of high-dimensional data spaces: implications for exploring gene and protein expression data. Nature reviews Cancer 2008;8:37-49

275. Ransohoff DF. Rules of evidence for cancer molecular-marker discovery and validation. Nature reviews Cancer 2004;4:309-14

276. Lehmann F, Lacombe D, Therasse P, Eggermont AM. Integration of Translational Research in the European Organization for Research and Treatment of Cancer 
Research (EORTC) Clinical Trial Cooperative Group Mechanisms. Journal of translational medicine 2003;1:2

277. Kriegsmann M, Casadonte R, Kriegsmann J, Dienemann H, Schirmacher P, Hendrik Kobarg J, et al. Reliable Entity Subtyping in Non-small Cell Lung Cancer by Matrixassisted Laser Desorption/Ionization Imaging Mass Spectrometry on Formalinfixed Paraffin-embedded Tissue Specimens. Molecular \& cellular proteomics : MCP 2016;15:3081-9

278. Michiels S, Koscielny S, Hill C. Prediction of cancer outcome with microarrays: a multiple random validation strategy. Lancet 2005;365:488-92

279. Patterson NH, Thomas A, Chaurand P. Monitoring time-dependent degradation of phospholipids in sectioned tissues by MALDI imaging mass spectrometry. Journal of mass spectrometry : JMS 2014;49:622-7

280. Goodwin RJ. Sample preparation for mass spectrometry imaging: small mistakes can lead to big consequences. Journal of proteomics 2012;75:4893-911

281. Goodwin RJ, Dungworth JC, Cobb SR, Pitt AR. Time-dependent evolution of tissue markers by MALDI-MS imaging. Proteomics 2008;8:3801-8

282. Dill AL, Eberlin LS, Costa $A B$, Ifa DR, Cooks RG. Data quality in tissue analysis using desorption electrospray ionization. Anal Bioanal Chem 2011;401:1949-61

283. Parson WB, Koeniger SL, Johnson RW, Erickson J, Tian Y, Stedman C, et al. Analysis of chloroquine and metabolites directly from whole-body animal tissue sections by liquid extraction surface analysis (LESA) and tandem mass spectrometry. Journal of mass spectrometry : JMS 2012;47:1420-8

284. Abdelmoula WM, Skraskova K, Balluff B, Carreira RJ, Tolner EA, Lelieveldt BP, et al. Automatic generic registration of mass spectrometry imaging data to histology using nonlinear stochastic embedding. Analytical chemistry 2014;86:9204-11

285. Schäfer K-C, Dénes J, Albrecht K, Szaniszló T, Balog J, Skoumal R, et al. In Vivo, In Situ Tissue Analysis Using Rapid Evaporative Ionization Mass Spectrometry. Angewandte Chemie International Edition 2009;48:8240-2

286. Golf O, Strittmatter N, Karancsi T, Pringle SD, Speller AV, Mroz A, et al. Rapid evaporative ionization mass spectrometry imaging platform for direct mapping from bulk tissue and bacterial growth media. Anal Chem 2015;87:2527-34

287. Gredell DA, Schroeder AR, Belk KE, Broeckling CD, Heuberger AL, Kim S-Y, et al. Comparison of Machine Learning Algorithms for Predictive Modeling of Beef Attributes Using Rapid Evaporative Ionization Mass Spectrometry (REIMS) Data. Scientific reports 2019;9:5721

288. Verplanken K, Stead S, Jandova R, Poucke CV, Claereboudt J, Bussche JV, et al. Rapid evaporative ionization mass spectrometry for high-throughput screening in food analysis: The case of boar taint. Talanta 2017;169:30-6

289. Balog J, Perenyi D, Guallar-Hoyas C, Egri A, Pringle SD, Stead S, et al. Identification of the Species of Origin for Meat Products by Rapid Evaporative Ionization Mass Spectrometry. Journal of Agricultural and Food Chemistry 2016;64:4793-800

290. Black C, Chevallier OP, Haughey SA, Balog J, Stead S, Pringle SD, et al. A real time metabolomic profiling approach to detecting fish fraud using rapid evaporative ionisation mass spectrometry. Metabolomics : Official journal of the Metabolomic Society 2017;13:153 
291. Rigano F, Mangraviti D, Stead S, Martin N, Petit D, Dugo P, et al. Rapid evaporative ionization mass spectrometry coupled with an electrosurgical knife for the rapid identification of Mediterranean Sea species. Anal Bioanal Chem 2019;411:6603-14

292. Guitton Y, Dervilly-Pinel G, Jandova R, Stead S, Takats Z, Le Bizec B. Rapid evaporative ionisation mass spectrometry and chemometrics for high-throughput screening of growth promoters in meat producing animals. Food Additives \& Contaminants: Part A 2018;35:900-10

293. Strittmatter N, Rebec M, Jones EA, Golf O, Abdolrasouli A, Balog J, et al. Characterization and Identification of Clinically Relevant Microorganisms Using Rapid Evaporative Ionization Mass Spectrometry. Analytical Chemistry 2014;86:6555-62

294. Strittmatter N, Jones EA, Veselkov KA, Rebec M, Bundy JG, Takats Z. Analysis of intact bacteria using rapid evaporative ionisation mass spectrometry. Chemical Communications 2013;49:6188

295. Cameron SJS, Bolt F, Perdones-Montero A, Rickards T, Hardiman K, Abdolrasouli A, et al. Rapid Evaporative Ionisation Mass Spectrometry (REIMS) Provides Accurate Direct from Culture Species Identification within the Genus Candida. Scientific reports 2016;6:36788

296. Bolt F, Cameron SJS, Karancsi T, Simon D, Schaffer R, Rickards T, et al. Automated High-Throughput Identification and Characterization of Clinically Important Bacteria and Fungi using Rapid Evaporative Ionization Mass Spectrometry. Analytical Chemistry 2016

297. Strittmatter N, Lovrics A, Sessler J, McKenzie JS, Bodai Z, Doria ML, et al. Shotgun Lipidomic Profiling of the NCI60 Cell Line Panel Using Rapid Evaporative Ionization Mass Spectrometry. Analytical Chemistry 2016;88:7507-14

298. Balog J, Szaniszlo T, Schaefer K-C, Denes J, Lopata A, Godorhazy L, et al. Identification of Biological Tissues by Rapid Evaporative Ionization Mass Spectrometry. Analytical Chemistry 2010;82:7343-50

299. Leff DR, St John ER, Takats Z. Reducing the margins of error during breastconserving surgery: Disruptive technologies or traditional disruptions? JAMA Surgery 2017;152:517-8

300. St John ER, Rossi M, Pruski P, Darzi A, Takats Z. Intraoperative tissue identification by mass spectrometric technologies. TrAC Trends in Analytical Chemistry 2016;85:2-9

301. Balog J, Kumar S, Alexander J, Golf O, Huang J, Wiggins T, et al. In Vivo Endoscopic Tissue Identification by Rapid Evaporative Ionization Mass Spectrometry (REIMS). Angewandte Chemie International Edition 2015;54:11059-62

302. Phelps DL, Balog J, Gildea LF, Bodai Z, Savage A, El-Bahrawy MA, et al. The surgical intelligent knife distinguishes normal, borderline and malignant gynaecological tissues using rapid evaporative ionisation mass spectrometry (REIMS). British journal of cancer 2018;118:1349-58

303. Tzafetas M, Mitra A, Paraskevaidi M, Bodai Z, Kalliala I, Bowden S, et al. The intelligent knife (iKnife) and its intraoperative diagnostic advantage for the treatment of cervical disease. Proceedings of the National Academy of Sciences 2020;117:7338-46 
304. Golf O, Muirhead LJ, Speller A, Balog J, Abbassi-Ghadi N, Kumar S, et al. XMS: CrossPlatform Normalization Method for Multimodal Mass Spectrometric Tissue Profiling. Journal of the American Society for Mass Spectrometry 2015;26:44-54

305. St John ER, Balog J, McKenzie JS, Rossi M, Covington A, Muirhead L, et al. Rapid evaporative ionisation mass spectrometry of electrosurgical vapours for the identification of breast pathology: towards an intelligent knife for breast cancer surgery. Breast Cancer Research 2017;19:59

306. Genangeli M, Heeren RMA, Porta Siegel T. Tissue classification by rapid evaporative ionization mass spectrometry (REIMS): comparison between a diathermic knife and $\mathrm{CO} 2$ laser sampling on classification performance. Anal Bioanal Chem 2019;411:7943-55

307. Sans M, Zhang J, Lin JQ, Feider CL, Giese N, Breen MT, et al. Performance of the MasSpec Pen for Rapid Diagnosis of Ovarian Cancer. Clinical chemistry 2019;65:674-83

308. Porcari AM, Zhang J, Garza KY, Rodrigues-Peres RM, Lin JQ, Young JH, et al. Multicenter Study Using Desorption-Electrospray-Ionization-Mass-Spectrometry Imaging for Breast-Cancer Diagnosis. Analytical Chemistry 2018;90:11324-32

309. Buck A, Heijs B, Beine B, Schepers J, Cassese A, Heeren RMA, et al. Round robin study of formalin-fixed paraffin-embedded tissues in mass spectrometry imaging. Anal Bioanal Chem 2018;410:5969-80

310. Ly A, Longuespee R, Casadonte R, Wandernoth P, Schwamborn K, Bollwein C, et al. Site-to-Site Reproducibility and Spatial Resolution in MALDI-MSI of Peptides from Formalin-Fixed Paraffin-Embedded Samples. Proteomics Clinical applications 2019;13:e1800029

311. Lopes JV, Bergerot CD, Barbosa LR, Calux N, Elias S, Ashing KT, et al. Impact of breast cancer and quality of life of women survivors. Revista brasileira de enfermagem 2018;71:2916-21

312. Beugels J, Cornelissen AJM, van Kuijk SMJ, Lataster A, Heuts EM, Piatkowski A, et al. Sensory Recovery of the Breast following Innervated and Noninnervated DIEP Flap Breast Reconstruction. Plastic and reconstructive surgery 2019;144:178e-88e

313. Calligaris D, Caragacianu D, Liu X, Norton I, Thompson CJ, Richardson AL, et al. Application of desorption electrospray ionization mass spectrometry imaging in breast cancer margin analysis. Proceedings of the National Academy of Sciences 2014;111:15184-9

314. Guenther S, Muirhead LJ, Speller AVM, Golf O, Strittmatter N, Ramakrishnan R, et al. Spatially Resolved Metabolic Phenotyping of Breast Cancer by Desorption Electrospray Ionization Mass Spectrometry. Cancer Research 2015;75:1828-37

315. Mason S, Manoli E, Poynter L, Alexander J, Paizs P, Adebesin A, et al. Mass spectrometry transanal minimally invasive surgery (MS-TAMIS) to promote organ preservation in rectal cancer. Surgical endoscopy 2019

316. Ironside AJ, Jones JL. Stromal characteristics may hold the key to mammographic density: the evidence to date. Oncotarget 2016;7:31550-62

317. DeFilippis RA, Chang H, Dumont N, Rabban JT, Chen YY, Fontenay GV, et al. CD36 repression activates a multicellular stromal program shared by high mammographic density and tumor tissues. Cancer discovery 2012;2:826-39 
318. Jones EA, Simon D, Karancsi T, Balog J, Pringle SD, Takats Z. Matrix Assisted Rapid Evaporative Ionization Mass Spectrometry. Analytical Chemistry 2019;91:9784-91

319. Pauling JK, Hermansson M, Hartler J, Christiansen K, Gallego SF, Peng B, et al. Proposal for a common nomenclature for fragment ions in mass spectra of lipids. PLOS ONE 2017;12:e0188394

320. Mao X, He J, Li T, Lu Z, Sun J, Meng Y, et al. Application of imaging mass spectrometry for the molecular diagnosis of human breast tumors. Scientific reports 2016;6:21043

321. Santoro AL, Drummond RD, da Silva IT, Ferreira SS, Juliano L, Vendramini PH, et al. In situ DESI - MSI lipidomic profiles of breast cancer molecular subtypes and precursor lesions. Cancer research 2020

322. Rauser S, Marquardt C, Balluff B, Deininger S-O, Albers C, Belau E, et al. Classification of HER2 Receptor Status in Breast Cancer Tissues by MALDI Imaging Mass Spectrometry. Journal of proteome research 2010;9:1854-63

323. DeHoog RJ, Zhang J, Alore E, Lin JQ, Yu W, Woody S, et al. Preoperative metabolic classification of thyroid nodules using mass spectrometry imaging of fine-needle aspiration biopsies. Proceedings of the National Academy of Sciences of the United States of America 2019;116:21401-8

324. Hishikawa D, Hashidate T, Shimizu T, Shindou H. Diversity and function of membrane glycerophospholipids generated by the remodeling pathway in mammalian cells. Journal of lipid research 2014;55:799-807

325. Mao L, Clark D. Molecular margin of surgical resections-Where do we go from here? Cancer 2015;121:1914-6

326. Caldwell RL, Gonzalez A, Oppenheimer SR, Schwartz HS, Caprioli RM. Molecular Assessment of the Tumor Protein Microenvironment Using Imaging Mass Spectrometry. Cancer Genomics - Proteomics 2006;3:279-87

327. McQuerry JA, Chang JT, Bowtell DDL, Cohen A, Bild AH. Mechanisms and clinical implications of tumor heterogeneity and convergence on recurrent phenotypes. Journal of molecular medicine (Berlin, Germany) 2017;95:1167-78

328. Friedman AA, Letai A, Fisher DE, Flaherty KT. Precision medicine for cancer with next-generation functional diagnostics. Nature Reviews Cancer 2015;15:747-56

329. Zhou L, Wang K, Li Q, Nice EC, Zhang H, Huang C. Clinical proteomics-driven precision medicine for targeted cancer therapy: current overview and future perspectives. Expert review of proteomics 2016;13:367-81

330. Armitage EG, Ciborowski M. Applications of Metabolomics in Cancer Studies. Advances in experimental medicine and biology 2017;965:209-34

331. Grapov D, Fahrmann J, Wanichthanarak K, Khoomrung S. Rise of Deep Learning for Genomic, Proteomic, and Metabolomic Data Integration in Precision Medicine. Omics : a journal of integrative biology 2018;22:630-6

332. Olivier M, Asmis R, Hawkins GA, Howard TD, Cox LA. The Need for Multi-Omics Biomarker Signatures in Precision Medicine. International journal of molecular sciences 2019;20

333. Griffin JL, Shockcor JP. Metabolic profiles of cancer cells. Nature Reviews Cancer 2004;4:551-61

334. Hannun YA, Obeid LM. Sphingolipids and their metabolism in physiology and disease. Nature Reviews Molecular Cell Biology 2017;19:175 
335. Ogretmen B. Sphingolipid metabolism in cancer signalling and therapy. Nature reviews Cancer 2018;18:33-50

336. Cheng C, Geng F, Cheng X, Guo D. Lipid metabolism reprogramming and its potential targets in cancer. Cancer communications (London, England) 2018;38:27

337. Mehlen P, Puisieux A. Metastasis: a question of life or death. Nature reviews Cancer 2006;6:449-58

338. Nicholson JK, Holmes E, Kinross JM, Darzi AW, Takats Z, Lindon JC. Metabolic phenotyping in clinical and surgical environments. Nature 2012;491:384-92

339. Alexander J, Gildea L, Balog J, Speller A, McKenzie J, Muirhead L, et al. A novel methodology for in vivo endoscopic phenotyping of colorectal cancer based on real-time analysis of the mucosal lipidome: a prospective observational study of the iKnife. Surgical endoscopy 2016

340. Talmadge JE, Fidler IJ. AACR centennial series: the biology of cancer metastasis: historical perspective. Cancer research 2010;70:5649-69

341. Obeid LM, Linardic CM, Karolak LA, Hannun YA. Programmed cell death induced by ceramide. Science 1993;259:1769-71

342. Hernandez-Corbacho MJ, Canals D, Adada MM, Liu M, Senkal CE, Yi JK, et al. Tumor Necrosis Factor-alpha (TNFalpha)-induced Ceramide Generation via Ceramide Synthases Regulates Loss of Focal Adhesion Kinase (FAK) and Programmed Cell Death. The Journal of biological chemistry 2015;290:25356-73

343. Kartal Yandim M, Apohan E, Baran Y. Therapeutic potential of targeting ceramide/glucosylceramide pathway in cancer. Cancer chemotherapy and pharmacology 2013;71:13-20

344. Nganga R, Oleinik N, Ogretmen B. Mechanisms of Ceramide-Dependent Cancer Cell Death. Advances in cancer research 2018;140:1-25

345. Murphy RC. Tandem Mass Spectrometry of Lipids: Molecular Analysis of Complex Lipids. Royal Society of Chemistry; 2015.

346. Murphy RC, Axelsen PH. Mass spectrometric analysis of long-chain lipids. Mass spectrometry reviews 2011;30:579-99

347. Hsu F-F. Complete structural characterization of ceramides as $[\mathrm{M}-\mathrm{H}]-$ ions by multiple-stage linear ion trap mass spectrometry. Biochimie 2016;130:63-75

348. Chong J, Yamamoto M, Xia J. MetaboAnalystR 2.0: From Raw Spectra to Biological Insights. Metabolites 2019;9:57

349. Rubbia-Brandt L, Giostra E, Brezault C, Roth AD, Andres A, Audard V, et al. Importance of histological tumor response assessment in predicting the outcome in patients with colorectal liver metastases treated with neo-adjuvant chemotherapy followed by liver surgery. Annals of oncology : official journal of the European Society for Medical Oncology 2007;18:299-304

350. Lee SY, Ju MK, Jeon HM, Jeong EK, Lee YJ, Kim CH, et al. Regulation of Tumor Progression by Programmed Necrosis. Oxidative medicine and cellular longevity 2018;2018:3537471

351. Park JH, Kim JH. Pathologic differential diagnosis of metastatic carcinoma in the liver. Clinical and molecular hepatology 2019;25:12-20

352. Park YS, Yoo CW, Lee SC, Park SJ, Oh JH, Yoo BC, et al. Lipid profiles for intrahepatic cholangiocarcinoma identified using matrix-assisted laser desorption/ionization 
mass spectrometry. Clinica chimica acta; international journal of clinical chemistry 2011;412:1978-82

353. Nagai K, Uranbileg B, Chen Z, Fujioka A, Yamazaki T, Matsumoto Y, et al. Identification of novel biomarkers of hepatocellular carcinoma by high-definition mass spectrometry: Ultrahigh-performance liquid chromatography quadrupole time-of-flight mass spectrometry and desorption electrospray ionization mass spectrometry imaging. Rapid communications in mass spectrometry : RCM 2020;34 Suppl 1:e8551

354. Gault CR, Obeid LM, Hannun YA. An overview of sphingolipid metabolism: from synthesis to breakdown. Advances in experimental medicine and biology 2010;688:1-23

355. Rego A, Trindade D, Chaves SR, Manon S, Costa V, Sousa MJ, et al. The yeast model system as a tool towards the understanding of apoptosis regulation by sphingolipids. FEMS yeast research 2014;14:160-78

356. Senkal CE, Salama MF, Snider AJ, Allopenna JJ, Rana NA, Koller A, et al. Ceramide Is Metabolized to Acylceramide and Stored in Lipid Droplets. Cell metabolism 2017;25:686-97

357. Omae F, Miyazaki M, Enomoto A, Suzuki M, Suzuki Y, Suzuki A. DES2 protein is responsible for phytoceramide biosynthesis in the mouse small intestine. The Biochemical journal 2004;379:687-95

358. Mizutani Y, Kihara A, Igarashi Y. Identification of the human sphingolipid C4hydroxylase, hDES2, and its up-regulation during keratinocyte differentiation. FEBS letters 2004;563:93-7

359. Schäfer K-C, Szaniszló T, Günther S, Balog J, Dénes J, Keserű M, et al. In Situ, RealTime Identification of Biological Tissues by Ultraviolet and Infrared Laser Desorption Ionization Mass Spectrometry. Analytical Chemistry 2011;83:1632-40

360. Basu SS, Regan MS, Randall EC, Abdelmoula WM, Clark AR, Gimenez-Cassina Lopez $B$, et al. Rapid MALDI mass spectrometry imaging for surgical pathology. NPJ precision oncology 2019;3:17

361. Calligaris D, Norton I, Feldman DR, Ide JL, Dunn IF, Eberlin LS, et al. Mass spectrometry imaging as a tool for surgical decision-making. Journal of mass spectrometry : JMS 2013;48:1178-87

362. Picard de Muller G, Ait-Belkacem R, Bonnel D, Longuespee R, Stauber J. Automated Morphological and Morphometric Analysis of Mass Spectrometry Imaging Data: Application to Biomarker Discovery. Journal of the American Society for Mass Spectrometry 2017;28:2635-45

363. Luberto C, Haley JD, Del Poeta M. Imaging with mass spectrometry, the next frontier in sphingolipid research? A discussion on where we stand and the possibilities ahead. Chemistry and physics of lipids 2019;219:1-14

364. Tata A, Woolman M, Ventura M, Bernards N, Ganguly M, Gribble A, et al. Rapid Detection of Necrosis in Breast Cancer with Desorption Electrospray Ionization Mass Spectrometry. Scientific reports 2016;6:35374

365. Calligaris D, Norton I, Feldman DR, Ide JL, Dunn IF, Eberlin LS, et al. Mass spectrometry imaging as a tool for surgical decision-making: Intraoperative mass spectrometry. Journal of Mass Spectrometry 2013;48:1178-87 
366. Sans M, Gharpure K, Tibshirani R, Zhang J, Liang L, Liu J, et al. Metabolic Markers and Statistical Prediction of Serous Ovarian Cancer Aggressiveness by Ambient Ionization Mass Spectrometry Imaging. Cancer research 2017;77:2903-13

367. Tafesse FG, Holthuis JC. Cell biology: A brake on lipid synthesis. Nature 2010;463:1028-9

368. Machala M, Prochazkova J, Hofmanova J, Kralikova L, Slavik J, Tylichova Z, et al. Colon Cancer and Perturbations of the Sphingolipid Metabolism. International journal of molecular sciences 2019;20

369. Aouali N, El Btaouri H, Dumontet C, Eddabra L, Malagarie-Cazenave S, Madoulet C, et al. Accumulation of lactosylceramide and overexpression of a PSC833-resistant P-glycoprotein in multidrug-resistant human sarcoma cells. Oncology reports 2011;25:1161-7

370. Dupre TV, Doll MA, Shah PP, Sharp CN, Siow D, Megyesi J, et al. Inhibiting glucosylceramide synthase exacerbates cisplatin-induced acute kidney injury. Journal of lipid research 2017;58:1439-52

371. Keegan CE, Hammer GD. Recent insights into organogenesis of the adrenal cortex. Trends in endocrinology and metabolism: TEM 2002;13:200-8

372. Bielinska M, Parviainen H, Kiiveri S, Heikinheimo M, Wilson DB. Review paper: origin and molecular pathology of adrenocortical neoplasms. Vet Pathol 2009;46:194-210

373. Sperone P, Ferrero A, Daffara F, Priola A, Zaggia B, Volante M, et al. Gemcitabine plus metronomic 5-fluorouracil or capecitabine as a second-/third-line chemotherapy in advanced adrenocortical carcinoma: a multicenter phase II study. Endocrine-related cancer 2010;17:445-53

374. Ducoulombier A, Cousin S, Kotecki N, Penel N. Gemcitabine-based chemotherapy in sarcomas: A systematic review of published trials. Critical reviews in oncology/hematology 2016;98:73-80

375. Ellis SR, Paine MRL, Eijkel GB, Pauling JK, Husen P, Jervelund MW, et al. Automated, parallel mass spectrometry imaging and structural identification of lipids. Nature methods 2018;15:515-8

376. Paine MRL, Poad BLJ, Eijkel GB, Marshall DL, Blanksby SJ, Heeren RMA, et al. Mass Spectrometry Imaging with Isomeric Resolution Enabled by Ozone-Induced Dissociation. Angewandte Chemie (International ed in English) 2018;57:10530-4

377. Niehaus M, Soltwisch J, Belov ME, Dreisewerd K. Transmission-mode MALDI-2 mass spectrometry imaging of cells and tissues at subcellular resolution. Nature methods 2019;16:925-31

378. Cameron SJS, Alexander JL, Bolt F, Burke A, Ashrafian H, Teare J, et al. Evaluation of Direct from Sample Metabolomics of Human Feces Using Rapid Evaporative Ionization Mass Spectrometry. Anal Chem 2019

379. Urabe T, Takahashi K, Kitagawa M, Sato T, Kondo T, Enomoto S, et al. Development of portable mass spectrometer with electron cyclotron resonance ion source for detection of chemical warfare agents in air. Spectrochimica acta Part A, Molecular and biomolecular spectroscopy 2014;120:437-44

380. Qiu SQ, Dorrius MD, de Jongh SJ, Jansen L, de Vries J, Schroder CP, et al. Microcomputed tomography (micro-CT) for intraoperative surgical margin assessment of breast cancer: A feasibility study in breast conserving surgery. European journal 
of surgical oncology : the journal of the European Society of Surgical Oncology and the British Association of Surgical Oncology 2018;44:1708-13

381. Asselin M, Kaufmann M, Wiercigroch J, Ungi T, Lasso A, Rudan J, et al. Navigated real-time molecular analysis in the operating theatre: demonstration of concept. SPIE; 2019.

382. Nauta SP, Poeze M, Heeren RMA, Porta Siegel T. Clinical use of mass spectrometry (imaging) for hard tissue analysis in abnormal fracture healing. Clinical chemistry and laboratory medicine 2020;58:897-913 


\section{Acknowledgments}

My name is on a cover but the achievement of this thesis needs to consider the collective effort of supervisors, collaborators and colleagues. I would like to globally thank all of you for your contribution to sharpen my scientific skills, to develop my knowledge of mass spectrometry, and to explore cancer pathologies. Beyond the simple acknowledgment, I am also taking with me your accents, your laughs, your jokes and your smiles (and some more tensed moments that made us certainly grow together but I am not elaborating on these here (:)). I may have try to reflect here a bit of what has inspired me by interacting with these diverse amazing personalities.

Ron. Ron is an inspiration for handling discussions and negotiations, for direction and management (and to echo a discussion from a recent M4I meeting in January 2020, I think management skills are an asset to bring people together to reach scientific achievements). I can disagree with the objectives but I can only acknowledge the global fairness of the supervision. Ron is not a boss, Ron is a leader by not only caring about big picture stuff but also technical difficulties. Ron is accessible. Ron gives input and suggests directions on projects. Ron is Dutch by his negotiation skills, by always having a positive constructive feedback, by his always-moving forwards attitude, by his ability to communicate and work with eclectic group of people. At some point, Ron asked me to do an iKnife demo for a dozen of priests and bishops. If this is not an illustration of how open to discussion and how translational interactions can get here, then I don't know what is. Ron, thank you for giving me the opportunity to do a PhD with your supervision. Thank you for your trust and your patience during that time. I may have tested some limits there. Thank you for this moment of feeling of freedom in scientific research.

Steven. Your enthusiasm for this project has been very important in the achievement of this thesis. Your clinical vision and your interest in the metabolic investigations that drove my interest for this PhD meant a lot. Your translational research approach contributed to the establishment of all these clinical collaborations, with the structure required to explore cancer pathologies, on liver and beyond! I appreciated a lot your stimulating and organized supervision and your comprehension of the efforts for this work and your support. I enjoyed your excitement about the results, your constructive and fair feedback on the storylines, and their valorisation by presentation requests. Steven, thank you for your supervision, your availability, and your generosity.

Bernd. Bernd, it is the first surgery I attended in MUMC+. Bernd, it is a talk in English at a national Dutch conference in front of my biggest audience. It was my fastest ever prepared abstract for submission (including emergency pathology examination), and a very effective oral presentation preparation. Bernd is exceptional. I really enjoy your supervision from study brainstorm to in vivo challenges. Your input has always been fair and constructive. I am really happy I got to contribute to research on head and neck cancers. Unfortunately, our work faced many challenges but I really hope we will manage to publish our manuscript, even if not included in this thesis. 
Tiffany. Tiffany, it is 4 years of supervision and some challenging set ups and implementations to reach first fresh ex vivo samples analyses in December 2016 and first presence of mobile Xevo to the operation room during a surgery in autumn 2018. I don't think I was really always easy-going and I think we evolved progressively together and we definitely improve our communication to deal with difficulties on a lot of projects! I would like to acknowledge in particular your patience and trust, especially regarding the last minute preparation for presentations, ASMS 2017 and OurCon 2017 (but best poster at MERLN symposium 2018, for the joke). Thank you for your calm and constructive feedback and your vision. I hope you appreciated the work and liked the end-messages, after our common efforts during these years to make this work. Thank you for your supervision! Tiffany, it was also a bit of student supervision and some special moments. Special thought about the planned rehearsal presentation of Laura, Zarina, Rodrigo and Josh on a morning of late June 2016. I cannot mention everyone, and as probably expected, I have to elaborate on the first. Jitske. I cannot qualify the way you are. Efficient. Fun. What a style also. I liked your punch, your comments, and your pieces of advice, perfect at the beginning of the PhD!

Benjamin. Thank you for giving me to possibility to dig into MSI for clinical research with you at the beginning of the PhD, I really enjoyed it. I would not have had the vision I had after without it. I learnt a lot, I like how the story was told, the contribution of each milestone considered and the potential next steps suggested. Thank you Benjamin!

Cathy. Cathy was always in a good mood. Working with you was great. Thank you very much for your help from ethics, patient inclusion to logistics and sample collection. I am very happy that you found a new working role that you like, surrounded by cat and dog that you love of course even more!

Marjolein Smidt, thank you for setting up the organisation to work on breast cancer! Christel Haeckens, Janine Lipsch-Crijns, Elly de Jong-Vrancken, Conny Starren-Goessens, Mieke Geurts, Femke Bruls thank you very much for your help with patient information! Sanne Engelen, Kristien Keymeulen, Litza Mitalas, Esther Heuts and all the other surgeons and AIOS, thank you for help with patient inclusion. Thank you for your reliability and efficiency! Somehow, if it turned out that this thesis ends with 2 chapters with breast cancer, it is in part thanks to your efficiency! And after the challenge to have the logistics working, I had a bit of difficulties to stop it! Sabeth, merci pour ton aide! Special thanks to Sanne Engelen for saving the record of the mastectomy! Special thanks to Marjolein Smidt and Charlotte de Jonge for the successful record of the breast-conserving surgery used in chapter 4 !

Laura Baijens, Bing Tan, Martin Lacko, Lauretta Vaassen, Auke van Mierlo, Annemiek Vanhouwe-Hermans and Els Bremer, thank you very much for helping with patient inclusion, logistics for tissue collection. Some challenging measurements with difficulties for electric supply and smoke aspiration, but thank you for your patience, and flexibility to enable the measurements.

I would like also to thanks the HBP surgeons for help with logistics, and Lieke Corpelijn for patient information. Special thanks to Marc Bemelmans also for the work on sarcomas! Judith de Vos-Geelen, thank you for your time and oncology expertise. 
Kristien Keymeulen. I cannot really pass by this section without a few words about Kristien Keymeulen. And at the same time, finding the right words for proper capture of the interaction is challenging. I am going to try to depict it, even if slightly romanticised. The following paragraph has been substantially romanticised. Readers have been warned. Kristien Keymeulen is, probably, certainly, without a doubt, by far, the nicest, the kindest and the most welcoming surgeon of MUMC+. Always in a good mood! If at some point I have considered seriously learning Dutch (I am shameful about the energy I have not put on this), it would have been to be able to communicate with Kristien Keymeulen. At the beginning, Kristien Keymeulen answered to me only in Dutch (I suppose, it sounded Dutch at least). Sometimes, without looking at me, so I could not even guess by face expression anything. Challenging. At that time (early 2017), regular communications by emails were not in place for Kristien Keymeulen (at least to follow my emails), and remembering surgical time-points was not an option. Also challenging. So, I got to learn Kristien Keymeulen weekly schedule and I was appearing by magic there and there in MUMC+ with updates and requests. And then, one day, on the morning of Wednesday March $28^{\text {th }} 2018$, I got a lollipop from Kristien Keymeulen. I informed Mari about this major improvement in our interaction right away. This was followed by substantial improvements. Some jokes. By the end of my contract, Kristien Keymeulen checked patient inclusion without reminder, wrote surgical time-points without request, assisted tissue selection for research at pathology, and performed in vivo measurement equipped with front light and GoPro on the head for one hour long surgery. Thank you Kristien Keymeulen!

Thanks to the clinical teams in the operation rooms who were very welcoming and who adapted their work to enable presence of the mass spectrometer and measurements during surgeries. Thank you to the OR complex teams in particular Rilana Duijzings and Ton Kolenburg for help with implementation, logistics and technical support. Arno Skrabanja, thank you for your speedy support to deal with ethical concerns!

Nina (everyone knows Nina!), Astrid, Inca, Drolaiz, Jolinda, Carmen, Prisca, Iryna, Lara, Kelly, Timo, Hendrik and all the AIOS pathology 2017-2020, thank you for your generous, and your sometimes less generous tissue contributions (and Timo's contributions were officially not substantially thinner over the summer 2018, despite national football considerations)! Mirjam, Tim, and all the pathology team members, thank you for your logistic coordination and sharing my excitement or my disappointment with tissue collection. Special thanks to Kelly for the adrenocortical carcinoma (chapter 5), to Prisca for the sarcoma (chapter 5), and to Lara for the cholangiocarcinoma (cover). Special thanks to lan for some livers, and Danny for some sarcomas.

Mari. Mari, it is the first iKnife burnt tissue examination in March 2017. It was also some challenging applications and examinations, with rare cases or very heterogeneous cases, making difficult to draw any conclusion. We had to be inventive and explorative to find interesting results, and every investigation was not successful. However, sometimes one case, one histological feature was enough to highlight a cool observation. How is the differentiation of the oral squamous cell carcinoma? Moderate! Now, we may have some nice molecular characterisation to some extend! Mari, thank you very much for these travels in oral cavity cancer and sarcoma pathologies! 
Loes. Loes, it is the first iKnife model built in July 2017. Loes, it is an impressive efficiency of precise pathology estimation of iKnife burns. It is also a huge organisation and we report 72 patients but the total amount of burns with mixed components is tremendous. Working with breast tissue was more challenging than the other tissues, and there was some struggle at the beginning but it worked out okay at the end! I really appreciated your feedback on the manuscripts and I am really happy that you like them! Thank you very much for making me discover breast pathology!

Heike. Heike, it is my first oral presentation in an international conference at Maastricht Pathology 2018. What a preparation for the abstract submission! It was the first time I experienced a feedback from Heike in March 2018, and it was not a high level one yet, that one came later! On that year, I was asked by some supervisors to improve my respect of deadlines (fair request after reading Tiffany's paragraph). Heike literally pushed the abstract submission deadline one week away on the initial day of the submission deadline. I read in this that you can always respect deadlines, as long as you can change the deadlines. I have no bias of interest to declare on this interpretation! Heike was on call to help with giving nice pieces of fresh liver. Heike was the last one standing to improve the last details of the liver manuscript. A lot of interesting investigations on liver (probably too much as the chapter was considered doubly twisted) but not only! Heike, it is also the tissue degradation manuscript 'it's pretty dead' with $95 \%$ of the examination performed during the UM hack 2019-2020. I cannot emphasize how grateful I am that I got the chance to work with Heike during this PhD. Heike, thank you for your histological pictures, your comments, your expertise, your time, and your pieces of advice.

Martin, Júlia, Adele, and all the iKnife consortium team members; thank you very much for your collaborative efforts. Honestly, with choosing to do this $\mathrm{PhD}$, I was partially hoping to contribute to this kind of work. I hope this work will contribute to make this type of technology useful to improve patient care. Special thought about my trip from Kingston to Toronto in April 2018 in Babar's car. Babar, thank you for the ride and the nice discussion.

Emrys Jones, I have being waiting for this DESI sprayer forever. This is just astonishing. Stable flow. No tip damage. Reliable. Beautiful images. Glomeruli resolution. Some might say that I was struggling with the PhD but the truth is I was waiting for this sprayer (I really say what I want here)! You enabled exciting biological investigations to reach applied messages I wanted for multiple projects. Thank you very much!

Júlia Balog, Steve Pringle, thank you for sharing your knowledge on the use of REIMS and Xevos for clinical studies, for ex and in vivo measurements. Thank you for your availability, very appreciable when you know that if something goes wrong, we may get have your assistance to deal with it shortly.

Kevin Hayden. It would be unfair to not thank Kevin Hayden for his training on the SimulTof, for sharing his mass spec expertise and his time with numerous emails but also phone-calls to get to know better and use the SimulTof. I wish I would have had managed to bring more value to the time we spent on this. Thank you Kevin! 
Simon, thank you for your efficient technical support!

Etienne. You know how people tend to ask in the hallway 'How are you?' without particularly giving insider information about their life and how it can be somehow perceived only as a polite salutation? So, one morning of summer 2017, I was in the sample prep lab certainly preparing some LeuEnk (Sigma Aldrich, Germany) solution in MS Grade isopropanol (Biolsolv, France) and Etienne was fixing something on the HTX (or more appropriately, the TM-sprayer, HTX technologies). Marta was there, doing something. Maybe I was actually just passing by, and actually, Marta was working. I guess it is better for the story if I was doing something. Does everyone know I am in the lab just to chat or not? Anyhow, the answer to that 'How are you?' question turned out not to be a short noneinsider answer. From there, our interaction became stronger and stronger and you helped me on a lot of things, sometimes a bit beyond your specific dedicated tasks. I would not say that we did not get our arguments. Our interactions could appear sometimes weirdly professional, or let's say very honest and straightforward. Vocal as you said. I tend to think I experienced with you the real, even raw Dutch straightforward attitude. I like that we could reach these borderline interactions for professional objectives in the work place, illustrating our trust in each other and the care we had about having the work done. Thank you for caring, thank you for your support, technical and not technical!

Frédéric. Frédéric cares way too much about this type of stuff so, purposely, I will not say much about Fred. No. I cannot do really that. That would be really-not nice, but I am not nice. That is a known fact. The problem with Fred is that we have been sharing the office for more than 4 years now. Fred is not my colleague or my friend, he is just part of my everyday life, even when not talking that much or at all. At some point, I could even more or less understand Fred without particularly talking. Some fun moments, some long lasting arguments. I am so thankful for all the things you did over these times including the not funny times. There are so many moments that I could recall but if I had to mention one here, that I could consider important, it would be that sunny Monday afternoon of June 2019 starting when Sanaz brought one topic to discussion about a situation she faced with a colleague or a friend, and your prompt personal comment on the subject!

Briete and Thiemo. Our first non-M4I officemates and we had our funny times. Thiemo was always accepting cookies and chocolates. Always. I even attended a Yankees game in NYC in June 2017 with Thiemo! Cool!

Sanaz. Sanaz arrived in the office in June 2017. While politeness and professional attitude were noticeable and appreciable from the very start, I realised the substantial attention and care Sanaz had in her observations and actions only on the late morning of Wednesday September $20^{\text {th }}$ 2017. I am grateful I got the chance to share your office along these years. Your dedication for your work and your objectives is an inspiration. You personality gave a positive note on every working day. Some days were extremely productive and some days were a lot of fun. I think we appreciated the same kind of humour but also we liked to have a bit more serious discussions. Thank you so much for everything Sanaz. I wish you the best in your achievements! Long live Sanaz! 
Renée. You do not know the socio-political business negotiations that were put in place to have Renée come from office K5.447 to office K5.449 in December 2017. Renée slightly chill and peaceable attitude is very enjoyable. It makes your jokes, comments and chitchat even more surprising and funny. One nice example that could be mentioned is probably you telling an interesting fact to make my stress go down before the talk at Maastricht Pathology 2018. Renée, it is also establishing the confidential agreement of K5.449! It was great to be with you in the office, thank you for these funny times, Renée!

Florian. Florian and I shared many perceptions on work, science or life in general, probably a shared French angle on things. I liked that you cared about things and that we could discuss them. I do not remember how I finally decided at some point of summer 2018 to go to UM Sports after your multiple recommendations since 2016. If at some point, I improved my balance that thanks to you, and a bit Jack too! Thank you Florian for the help for finalizing this thesis and everything. What do you mean there are no new M4I templates? What about a carnival edition?

Maxime. Maxime, it is the tranquil strength. A clear mind, an organized agenda and probably a precisely checked bank account and food diet! With his own perceptions and goals. Not that much under influenced. Not to be too much disturbed. It was fun having you around, thank you for the good moments, Maxime!

Nina. Nina's birthday parties (I have at least two in mind, so parties) and Nina's departure party were probably among the nights I had the most fun here. What a phenomenon Nina! What an expressive face! Can say I am going crazy like Nina tonight? No sure I can though, but just for the spirit!

Jian-Hua. Jian-Hua arrived at M4I in September 2017 (“What's your name again?”). You brought with you your Chinese intonations, which made your sentences become classics such as "it is too sweet, I cannot handle it", "it's fine", "and that's it". However, I like at bit less your "I am shy" and "I am polite" while you are kind of taking a lot of attention and rude! I am tremendously thankful for all the help from pathology scanning to bad Rapiflex updates. But beyond that, I think the influence I got from you, is that without using that much diplomatic tact, your Chinese inspired short sentences give you a strong dynamism in what you do. A straightforward and valuable direct attitude and somehow that works, sometimes to my personal surprise. You know, I am usually more like unclear, subtle, and lost in complexity and no one gets me (I know how to sell myself also). Now, I am trying to reduce the length of my sentences (for scientific writing, but here it is personal writing!). To be more direct. Clearer. Less diplomatic. Active. However, I am not sure it works that well with me. What is even more interesting is how people can react to it. Somehow, sometimes, it is too fast and surprising, and people are destabilized and don't know how to react, which can, then, not always lead to a good resolution. The rude strategy is to try but to use with caution. However, sometimes, a complementary diplomatic approach can save some deals. I would take for example the arrival at the restaurant A Nova Estrela in Lisbon, on August $19^{\text {th }} 2019$ and the negotiation to merge 2 tables with separate services for 9 . Out of the rude topic, I have to report your aptitude to get someone to open up to you in a very short amount of time (e.g. the quais de Seine interview, December $21^{\text {st }} 2019$ ). Lastly, I have to 
mention the evening we pretended I was your supervisor so I could join a Chinese diner with Lin, Chang and you! "Anyway, Ron is not interested in Chinese food, he just eats sandwiches. What? It's true!" I totally distance myself from this statement!

Lin, Yuandi, Peilang, Ling-Ling, Lichuan (mostly referred and known as Ling-Ling's boyfriend!), Chang, Peilong, Hongxia, Xinwei, Xiuxiang. Thank you for the food. A lot of food! Hot pots. Dumplings. A lot of meals that I ate but I don't remember their names. Thank you for the fun. Happy I got to finally play the mahjong I brought back from Baisha 10 years ago. Peilang, thank you for your very smiling "Good morning Pmax"!

Thank you Paul, Andrew, Rob and Sanaz for organizing the lab outing 2018. I was happy to contribute to that! A bit disappointed that most of the alpacas I was in charge of hiding were not successful catches, but it was a fun organisation!

Hans, Carmen, Peter, Frank and Nanoscopy team, it was nice interacting with you, and to do a bit of electro-microscopy, thank you! Peter, love your talk at Maastricht Pathology 2018 ! Love your comments at the M4I meetings!

Abril became mi corazón after I went to Colombia in August 2018 but Abril was already the safe contact in case of running late experiments way before that! Than you Abril!

Romy, Rob, Evie (and your nice chitchats!), Caitlin, Robert, Anne, Marjolein, Ralph, thank you very much for your nice help with patient inclusion, medical Dutch assistance and understanding clinical data organisation! Audrey, Annet, Jungfang, Mirjam, Hong, Sander, Frank, Hans, Kees, Chantal, Tessa, Loes, Zita, Janine, Rianne, Merel, Sebastiaan, Jacqueline, Sadé, Aurelia, Sabine, Liyanne, David, Marissa, Yvonne (and the yoghurt story!), Claire, Bas and Mo, it was fun working and spend some time with you!

Imke and Wouter, thank you very much for joining in the head and neck project. I am very happy that we will be able report the story I wanted, and that would not have been possible without you. Thank you for your trust and efforts. Natasja Kisters, thank you very much for dealing with the biosafety regulations and requests to enable the head and neck experiment, after our multiple exchanges and meetings in summer 2019.

I want to thank all the M4I team members, past and present (I cannot make a personal note for everyone otherwise this will never be printed without too many typos); Naomi, Philippe, Sylvia, Stephanie, Klára, Michele, Jo, Fabian, Roel, Isabeau, Lucia, Charles, Shane, Ian, Joel, Gert, Frans, Rob, Maarten, Helen, Aljoscha, Loes, Kasper, Sanne, Keely, Marty, Pariz, Benjamin, Ali, Ana and all the ones that I have not mentioned. Caro. Martha (Thank you Martha for dealing with the Vrijthof fountain incident). Including guests. Your ideas, your works and your personalities are inspirations to give the better of yourself in what you do, every day. Very motivating! I was pleased to be part of the group to follow your challenges, your successes and your evolutions. Thank you for your contribution, for your help in the realisation of this thesis. 
Ronny, thank you for dealing with my numerous orders over these years and your positive feedbacks on my spams!

Laura and Eva, I am very happy I got to contribute to your project, that was very nice to work with you, thank you!

Bryn. Bryn is the nicest guy. Your dedication for work at some points was crazy. Maybe I got crazy but you were crazy first! It was so nice having you around. Thank you for everything. Take care of yourself Bryn! I wish you a lot of happiness, Brynou!

Darya. I love that Darya had 9 students at some point. I really enjoy having you around, your personality, your kick-ass attitude, even if a bit too punchy sometimes; but if it is because my jokes are not good then that's fine.

Anjusha, I love your enthusiasm when you present during group meeting but also when you discuss about life and plans outside of the group meeting! Thank you for the nice chats and food gatherings!

Lennart, you remember the key of my bike locker that broke in the bike locker that Octopus night? Because I don't. Thank you for acclimating me to Maastricht at the beginning. Thank you for all the cakes!

Lieke, thank you for your reliable help with your analytical expertise! It was great having you around, especially sharing the office on the 3rd floor! You were also a great PhD representative despite what some French revolutionary spirits might have let believe at some point! :)

Pieter, thank you for the entertaining evening discussions, the side test experiments, and the movie viewing on August 26th 2017!

Andrew and his not-that-famous eyebrows-based deep-voiced "what's up?" Britt can you do a meme out of that? $:$

Britt and her famous chin-based silenced hello sign. Britt was the last one standing after the $3^{\text {rd }}$ floor never dies celebration on Thursday March 16th 2017! Your laugh!

Marta and Bea, your delicate and soft Spanish vibes were bighting up my mood. Thank you for the nice chats. Bea's "P-maaaax" is difficulty forgettable. Marta's sangria is missing!

Mirella and Berta, I got rare opportunities to notice your calm attitude in discussions and meetings. Never increasing your tone, never losing your temper, even slightly. A tentative to make a point never requires rising the voice. I guess sometimes I tend either to pop up the volume too fast or to drop the point I try to make at bit too fast. However, I plan to adopt your inspiring attitude in the next future! What? I can be calm! I am calm! 
Thank you to my landlord Andrew. It was a challenge to become your tenant (some concern about my profile picture of me disguised as David Bowie apparently?(:)) in winter 2017 but I was convinced I would like the place, despite its downsides. I love this place. There is something charming and special with the combination of the view on the Mass and on the rocks of St Pietersburg's mountain, the sound of flowing water, of the birds, of the frogs in the early summer nights, and of Fun Valley's electronic music in the late summer nights. This place contributed to get me the rest required to have the energy for the PhD and to write during quarantine, thank you!

Special thanks to my previous study colleagues, professors and supervisors who have participated in building my professional path, and who have experienced my collaboration or my supervision.

Special thanks to my sister, parents, family and friends who have supported me over this PhD period. Thank you very much for being there for me. Thank you for giving me opportunities to get out of the PhD research for fresh and recharging breaks.

Most of the research presented in this thesis was performed at the M4I institute and at Maastricht University Medical Centre (MUMC+) and was financially supported by MUMC+ and by the Dutch Province of Limburg as part of the LINK program.

Chapter 2. BB thanks the European Union (ERA-NET: TRANSCAN 2), ITEA and RVO (ITEA 151003/ITEA 14001) for their financial support. I am thankful to Stacey Meulenberg for her excellent technical assistance. I am thankful to Lindsay Hewitt (Pathology Department) from MUMC+ for providing the tissues and pathological annotations for figures 1 and 3 . I am thankful to Justin Baker for editing the manuscript.

Chapter 3 research performed at Queen's University has been made possible thanks to the support of the Britton Smith Chair in Surgery (to JR). I thank Lina Chen, Julie Wallis and Kathryn Logan (Queen's) for assistance with clinical sample collection. 


\section{List of publications}

Chapter 2

Mass spectrometry imaging for clinical research - latest developments, applications, and current limitations

Pierre-Maxence Vaysse., Ron M.A. Heeren, Tiffany Porta and Benjamin Balluff

The Analyst 2017 142(15): 2690-2712.

Chapter 3

Multi-site development and testing of a breast cancer recognition platform based on rapid evaporative ionization mass spectrometry

Martin Kaufmann and Pierre-Maxence Vaysse, Adele Savage, Ala Amgheib, András Marton, Eftychios Manoli, Loes F.S. Kooreman, Natasja Janssen, Sonal Varma, Shaila Merchant, Cecil Jay Engel, Steven W.M. Olde Damink, Marjolein L. Smidt, Sami Shousha, Hemali Chauhan, Gabor Fichtinger, Steven D. Pringle, John F. Rudan, Ron M.A. Heeren, Zoltán Takáts, Júlia Balog and Tiffany Porta Siegel

In preparation

\section{Chapter 4}

Stromal vapours for real-time molecular guidance of breast-conserving surgery

Pierre-Maxence Vaysse, Loes F.S. Kooreman, Sanne M.E. Engelen, Bernd Kremer, Steven W.M. Olde Damink, Ron M.A. Heeren, Marjolein L. Smidt, Tiffany Porta Siegel

Scientific Reports, Accepted October 2020

\section{Chapter 5}

Real-time lipid patterns to classify viable and necrotic liver tumours

Pierre-Maxence Vaysse, Heike I. Grabsch, Mari F.C.M. van den Hout, Marc H.A. Bemelmans, Ron M. A. Heeren, Steven W. M. Olde Damink, Tiffany Porta Siegel

Under revisions 